\title{
Estimated Water Use, By County, in North Carolina, 1995
}

By Douglas A. Walters

U.S. GEOLOGICAL SURVEY

Open-File Report 97-599

Prepared in cooperation with the

Division of Water Resources of the

North Carolina Department of Environment and Natural Resources

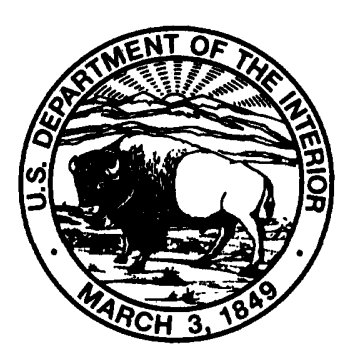




\section{U.S. DEPARTMENT OF THE INTERIOR \\ BRUCE BABBITT, Secretary}

U.S. GEOLOGICAL SURVEY

Mark Schaefer, Acting Director

The use of firm, trade, and brand names in this report is for identification purposes only and does not constitute endorsement by the U.S. Geological Survey

For additional information write to:

District Chief

U.S. Geological Survey

3916 Sunset Ridge Road

Raleigh, NC 27607
Copies of this report can be purchased from:

U.S. Geological Survey

Information Services

Box 25286

Federal Center

Denver, CO 80225 


\section{CONTENTS}

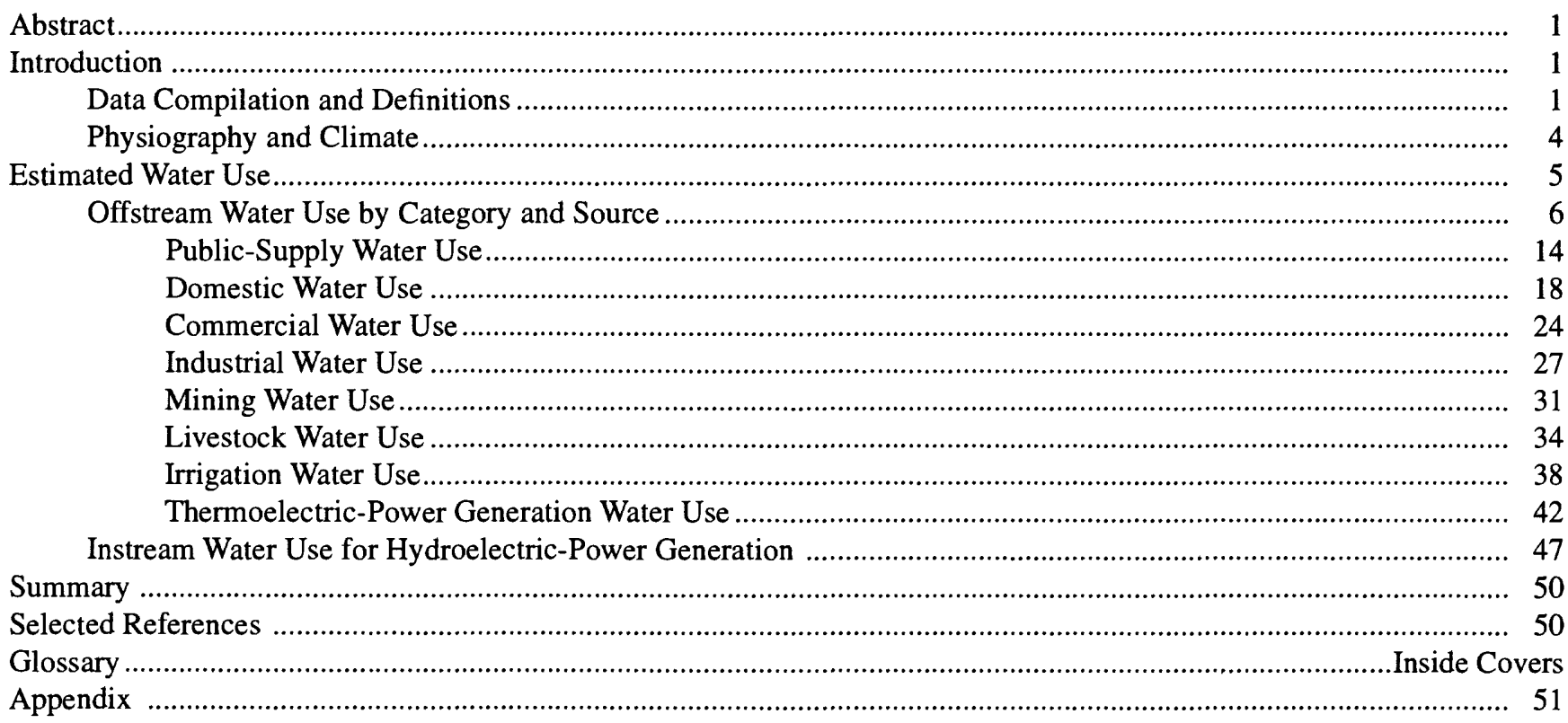

\section{FIGURES}

1. Map showing counties and physiographic provinces in North Carolina

2. Flow chart showing source, use, and disposition of an estimated 9,286 million gallons per day of fresh and saline water withdrawals in North Carolina, 1995.

3. Graph showing water withdrawals, by water-use category, in North Carolina, 1995.

4. Pie chart and graph showing water withdrawals, by source, and water-use category and source, in North Carolina, 1995.

5. Maps showing water withdrawals, by county, in North Carolina, 1995

6. Maps showing total population and population served by public suppliers, by county, in North Carolina, $1995 \ldots . .14$

7. Pie chart showing sources of public-supply withdrawals, and graph showing public-supply deliveries, by water-use category, in North Carolina, 1995

8. Maps showing public-supply withdrawals, by county, in North Carolina, 1995 ......................................... 19

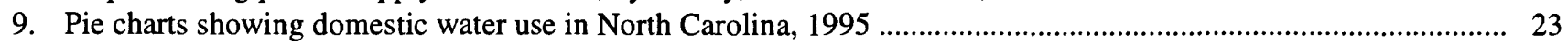

10-12. Maps showing:

10. Population reliant on self-supplied water for domestic use, by county, in North Carolina, 1995 ................... 23

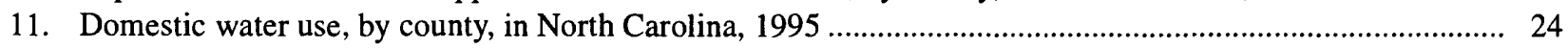

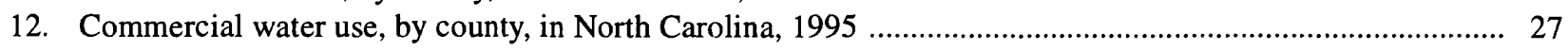

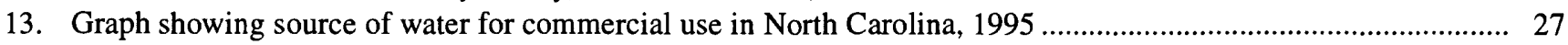

14. Graph showing source of water for industrial use in North Carolina, 1995 .................................................... 30

15. Maps showing industrial water use, by county, in North Carolina, 1995 ...................................................... 30

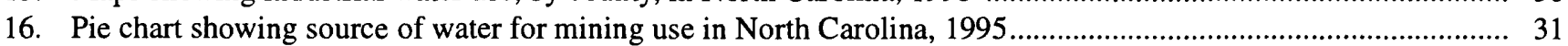

17. Map showing total withdrawals for mining water use, by county, in North Carolina, 1995 ................................... 31

18. Pie chart and graph showing source of water for livestock use in North Carolina, 1995 ................................... 34

19. Maps showing livestock water use, by county, in North Carolina, 1995 ......................................................... 38

20. Graph and pie chart showing irrigation water use, by source and irrigation type, in North Carolina, 1995 ............. 39

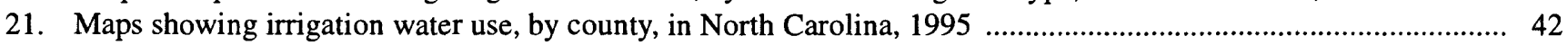


22. Maps showing total withdrawals for thermoelectric-power generation and power generated, by county, in North Carolina, 1995

23. Pie charts showing thermoelectric-power generation, by power type, in North Carolina, 1995

24. Maps showing total instream water use for hydroelectric-power generation and power generated, by county, in North Carolina, 1995

\section{TABLES}

1. Water-use data collection, by category, in North Carolina, 1995

2

2. Total water withdrawals, by source and water-use category, in North Carolina, 1995

3. Total water withdrawals, by water-use category and county, in North Carolina, 1995

4. Total water withdrawals, by source, use, and county, in North Carolina, 1995.

5. Public-supply water use, by source, water-use category, and county, in North Carolina, 1995.

6. Domestic water use, by county, in North Carolina, 1995.

7. Commercial water use, by county, in North Carolina, 1995 .

8. Industrial water use, by county, in North Carolina, 1995.

9. Mining water use, by county, in North Carolina, 1995

10. Livestock water use, by county, in North Carolina, 1995 .

11. Irrigation water use, by county, in North Carolina, 1995

12. Thermoelectric-power generation water use and power generated, by county, in North Carolina, 1995 .

13. Hydroelectric-power generation water use, by county, in North Carolina, 1995

\section{CONVERSION FACTORS}

\begin{tabular}{rcl}
\hline Multiply & by & To obtain \\
\hline & Flow & \\
gallons per day (gal/d) & 0.003785 & cubic meter per day \\
& 0.04381 & cubic meter per second \\
million gallons per day (Mgal/d) & Energy & \\
kilowatt-hour $(\mathrm{kWh})$ & $3,600,000$ & joule \\
\hline
\end{tabular}




\title{
Estimated Water Use, by County, in North Carolina, 1995
}

\author{
By Douglas A. Walters
}

\begin{abstract}
Data on water use in North Carolina were compiled for 1995 as part of a cooperative agreement between the U.S. Geological Survey and the Division of Water Resources of the North Carolina

Department of Environment and Natural Resources. Data were compiled from a number of Federal, State, and private sources for the offstream water-use categories of public supply, domestic, commercial, industrial, mining, livestock, irrigation, and thermoelectric-power generation. Data also were collected for instream use from hydroelectric facilities.

Total withdrawals (fresh and saline) during 1995 were an estimated 9,286 million gallons per day for the offstream water-use categories. About 94 percent of the water withdrawn was from surface water. Thermoelectric-power generation accounted for 80 percent of all withdrawals. Instream water use for hydroelectric-power generation totaled about 56,400 million gallons per day. Each water-use category is summarized in this report by county and source of water supply.
\end{abstract}

\section{INTRODUCTION}

North Carolina has seemingly abundant water resources; however, population growth and increases in municipal, industrial, agricultural, and recreational water use have placed increasing demands on the State's water supply. Although years of plentiful rainfall generally provide adequate water supplies to meet most demands during years of drought, conflicts may arise in some places that require rationing and other conservation measures to ensure that water supplies are adequate to meet demands and that water quality remains unaffected during periods of low flow. In order to assist in planning and management of the State's water resources, estimates of water use are produced.

The North Carolina Water-Use Program is part of the National Water-Use Information Program operated by the U.S. Geological Survey (USGS). The National Water-Use Information Program is a cooperative program with State and local governments designed to collect and disseminate water-use information to a wide variety of government agencies and private organizations. The USGS, in cooperation with the Division of Water Resources (DWR) of the North Carolina Department of Environment and Natural Resources (DENR) (formerly the North Carolina Department of Environment, Health, and Natural Resources), surveyed and compiled ground- and surface-water use data in North Carolina for 1995. These data were compiled from a variety of Federal, State, and private sources for the offstream water-use categories of public supply, domestic, commercial, industrial, mining, livestock, irrigation, and thermoelectric-power generation. Data also were collected for instream use from hydroelectric facilities.

The purpose of this report is to provide an estimate of water use, by county, in North Carolina for 1995. Data are summarized for nine categories of water use as required for the USGS circular on water use in the United States, which is published every 5 years. Water-use estimates for instream and offstream use are summarized, as well as water use by source-ground water and surface water.

\section{Data Compilation and Definitions}

Compilation methods and standards used in this study are in the document titled "Guidelines for 
Preparing U.S. Geological Survey Water-Use Estimates in the United States for 1995" (Crompton and Solley, 1995). Estimates for water withdrawn by category were obtained from a variety of sources. This information is summarized by category in table 1 . Whenever metered data were not available, estimated values were used. For clarity, water-use values in the text are rounded and percentages are expressed as integers. These values may not add to totals because of rounding.
Public-supply water use is defined as water withdrawn by public and private suppliers that funish water for at least 25 people, or that have a minimum of 15 hookups. This category includes water delivered for domestic, commercial, industrial, and thermoelectricpower generation use and for other users who do not supply their own water needs. Domestic self-supplied water use is limited to individual households that are not served by public-supply systems. Commercial selfsupplied withdrawals refer to water withdrawn by

Table 1. Water-use data collection, by category, in North Carolina, 1995

[DENR, North Carolina Department of Environment and Natural Resources; DWR, Division of Water Resources; LWSP, Local Water Supply Plan; DEH, Division of Environmental Health; PWSS, Public Water Supply Section; USGS, U.S. Geological Survey; USDOC, U.S. Department of Commerce; gal/d, gallons per day; NCDOC, North Carolina Department of Commerce; B/IDD, Business/Industry Development Division; NCGS, North Carolina Geological Survey; LQS, Land Quality Section; DWQ, Division of Water Quality; NCSU, North Carolina State University; AES, Agriculture Extension Service; NCDA, North Carolina Department of Agriculture; ASS. Agricultural Statistics Section; ANR, Aquaculture and Natural Resources; BAE, Biological and Agricultural Engineering; BC, Bureau of the Census; PID, Plant Industry Division; CS, Crop Science; TCNC, Turfgrass Council of North Carolina]

\begin{tabular}{cc}
\hline Data sources & Data collection-Type, method, and analysis \\
\hline & PuBuC SUPPLY \\
\hline
\end{tabular}

DENR, DWR 1992-93 LWSP database and hardcopy files.

DENR, DEH, PWSS Public water-supply database. Community water systems, noncommunity water systems, and data on population served, and source of water to supplement site data from DENR, DWR.

USGS Compile and aggregate data by county.

\begin{tabular}{ll}
\hline & \multicolumn{1}{c}{ DOMnsnc } \\
\hline DENR, DWR & 1992-93 LWSP database and hardcopy files. Municipal public-supply facility information on water use. \\
DENR, DEH, PWSS & $\begin{array}{c}\text { Public water-supply database. Community water systems and data on population served, and source of water } \\
\text { to supplement site data from the LWSP. }\end{array}$ \\
USDOC & $\begin{array}{l}\text { Population within a county. } \\
\text { Water-use coefficient of } 70 \mathrm{gal} / \mathrm{d} \text { per person. Calculation per county: (county population) - (population served } \\
\text { by public suppliers) } \mathrm{x}(70 \mathrm{gal} / \mathrm{d}) \text {. Compile and aggregate data by county. }\end{array}$
\end{tabular}

\begin{tabular}{ll}
\hline COMMERCIAL \\
\hline DENR, DWR & $1992-93$ LWSP database and hardcopy files. Municipal public-supply facility information on water use. \\
DENR, DEH, PWSS & $\begin{array}{l}\text { Public water-supply database. Noncommunity water systems, and data on population served, and source of } \\
\text { water to supplement site data from the LWSP. }\end{array}$
\end{tabular}

USGS Water-use coefficients for various commercial users. Compile and aggregate data by county.

\section{INDUSTRIAL}

DENR, DWR

1992-93 LWSP database and hardcopy files. Municipal public-supply facility information on water use.

NCDOC, B/IDD Identification of industries from "North Carolina Manufacturing Firms, 1995 Directory."

USGS

Mail survey of 500 industries. Statistical analysis of water-use estimates for industries using survey results.

Telephone conversations with industry personnel. Compile and aggregate data by county. 
Table 1. Water-use data collection, by category, in North Carolina, 1995-Continued

[DENR, North Carolina Department of Environment and Natural Resources; DWR, Division of Water Resources; LWSP, Local Water Supply Plan; DEH, Division of Environmental Health; PWSS, Public Water Supply Section; USGS, U.S. Geological Survey; USDOC, U.S. Department of Commerce; gal/d, gallons per day; NCDOC, North Carolina Department of Commerce; B/IDD, Business/Industry Development Division; NCGS, North Carolina Geological Survey; LQS, Land Quality Section; DWQ, Division of Water Quality; NCSU, North Carolina State University; AES, Agriculture Extension Service; NCDA, North Carolina Department of Agriculture; ASS, Agricultural Statistics Section; ANR, Aquaculture and Natural Resources; BAE, Biological and Agricultural Engineering; BC, Bureau of the Census; PID, Plant Industry Division; CS. Crop Science; TCNC. Turfgrass Council of North Carolina]

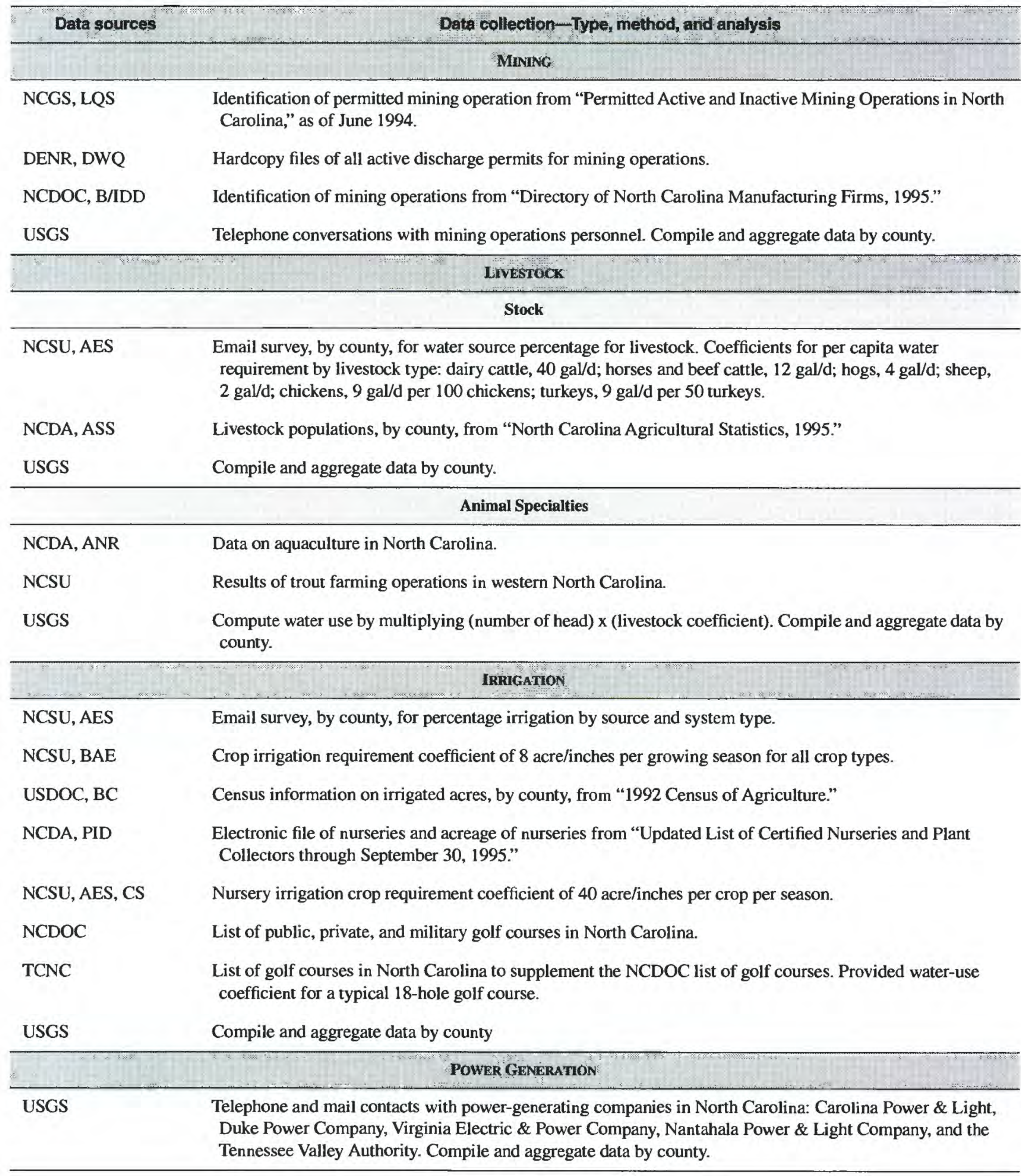


hotels, restaurants, office buildings, churches, and other commercial facilities and institutions, including universities and military installations. Industrial selfsupplied water use is water used for industrial purposes in the manufacturing of products. Mining water use consists of withdrawals for use in the extraction of minerals and to wash, sort, and transport mineral products. Mining water use does not include water that is removed by dewatering of excavation pits. The livestock category includes water used for livestock watering, feed lots, dairy operations, and fish farming. Irrigation water use is defined as the artificial application of water to lands to assist in the growing of crops and pastures or to maintain vegetative growth in commercial nurseries and recreational lands, such as parks and golf courses. Water used for the power generation category includes the instream use by 38 hydroelectric powerplants and offstream withdrawals by 18 fossil-fuel and 3 nuclear facilities in North Carolina. This category is divided into water use for hydroelectric and thermoelectric power (fossil-fuel and nuclear facilities). Water use for hydroelectric-power generation is the only category in this report considered as an instream water use.

Some county-level data are based on site-specific data collected by various Divisions within the DENR. Water-use data for domestic, commercial, industrial, livestock, and irrigation were not available for all sites within the State. Therefore, estimates were calculated using coefficients obtained from knowledgeable sources (table 1) applied to survey, census, or agricultural statistical data.

\section{Physiography and Climate}

The physiography of North Carolina is diverse. The State has three physiographic provinces-the Blue Ridge in the west, the Piedmont in the central part of the State, and the Coastal Plain in the east (fig. 1). The Blue Ridge consists of mountainous terrain giving way to the gently sloping, hilly terrain of the Piedmont. The Coastal Plain is flat and low-lying with little topographic relief. Streams and rivers along the eastern slope of the Blue Ridge flow to the Atlantic Ocean, and streams and rivers along the western slope flow to the Ohio and Tennessee River Basins in adjacent States.

The division between the western and eastern watersheds of the Blue Ridge is known as the Tennessee Valley Divide (fig. 1). The dominant source of water varies by Provinces; surface water is the primary source in the Blue Ridge and Piedmont Provinces, and ground water is the primary source in the Coastal Plain Province.

North Carolina has a mild, humid climate and ranks sixth in the Nation in average annual precipitation with 49.3 inches. In 1995, the State experienced a higher-than-normal precipitation amount of 54.9 inches (National Atmospheric and Oceanic Administration, 1996). A water-budget study in eastern North Carolina indicated that as much as 61 percent of

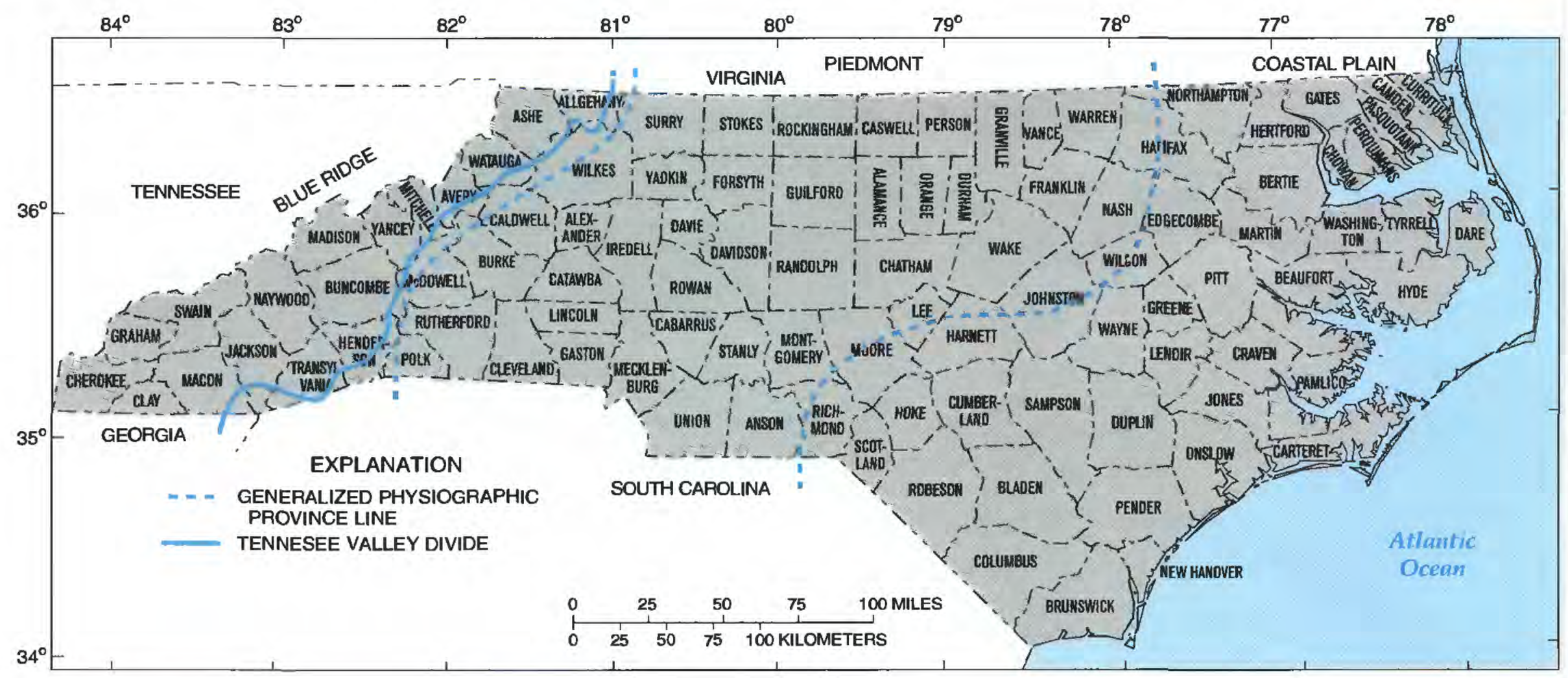

Figure 1. Counties and physiographic provinces in North Carolina. 
the annual precipitation is returned to the atmosphere by evapotranspiration, and about 39 percent recharges the ground- and surface-water systems (Winner and Simmons, 1977).

\section{ESTIMATED WATER USE}

In 1995, total (fresh and saline) offstream water use in North Carolina was estimated at 9,286 million gallons per day (Mgal/d) (table 2). A flow diagram illustrating source, use, and disposition of 1995 water withdrawals in North Carolina is shown in figure 2. Offstream water use refers to all water "withdrawn or diverted from ground- or surface-water source for public water supply, industry, irrigation, livestock, thermoelectric-power generation, and other uses. It is sometimes referred to as off-channel use or withdrawal," (Solley and others, 1993). Of the total withdrawals in 1995,80 percent, or 7,417 Mgal/d, was

Table 2. Total water withdrawals, by source and water-use category, in North Carolina, 1995

[Values in million gallons per day]

\begin{tabular}{|c|c|c|c|c|c|c|c|c|c|}
\hline Water source & $\begin{array}{l}\text { Public } \\
\text { supply }\end{array}$ & $\begin{array}{l}\text { Domes- } \\
\text { tic }\end{array}$ & $\begin{array}{l}\text { Com- } \\
\text { mercial }\end{array}$ & $\begin{array}{l}\text { Indus- } \\
\text { trial }\end{array}$ & Mining & $\begin{array}{l}\text { Live- } \\
\text { stock }\end{array}$ & $\begin{array}{l}\text { Irriga- } \\
\text { tion }\end{array}$ & $\begin{array}{l}\text { Thermo- } \\
\text { electric }\end{array}$ & Total \\
\hline Ground water & 136.38 & 171.53 & 7.31 & 61.10 & 11.69 & 89.48 & 57.29 & 0.11 & 534.89 \\
\hline Surface water & 632.85 & 0.00 & 0.29 & 307.94 & 4.33 & 207.10 & 181.28 & 7.416 .89 & $8,750.68$ \\
\hline
\end{tabular}

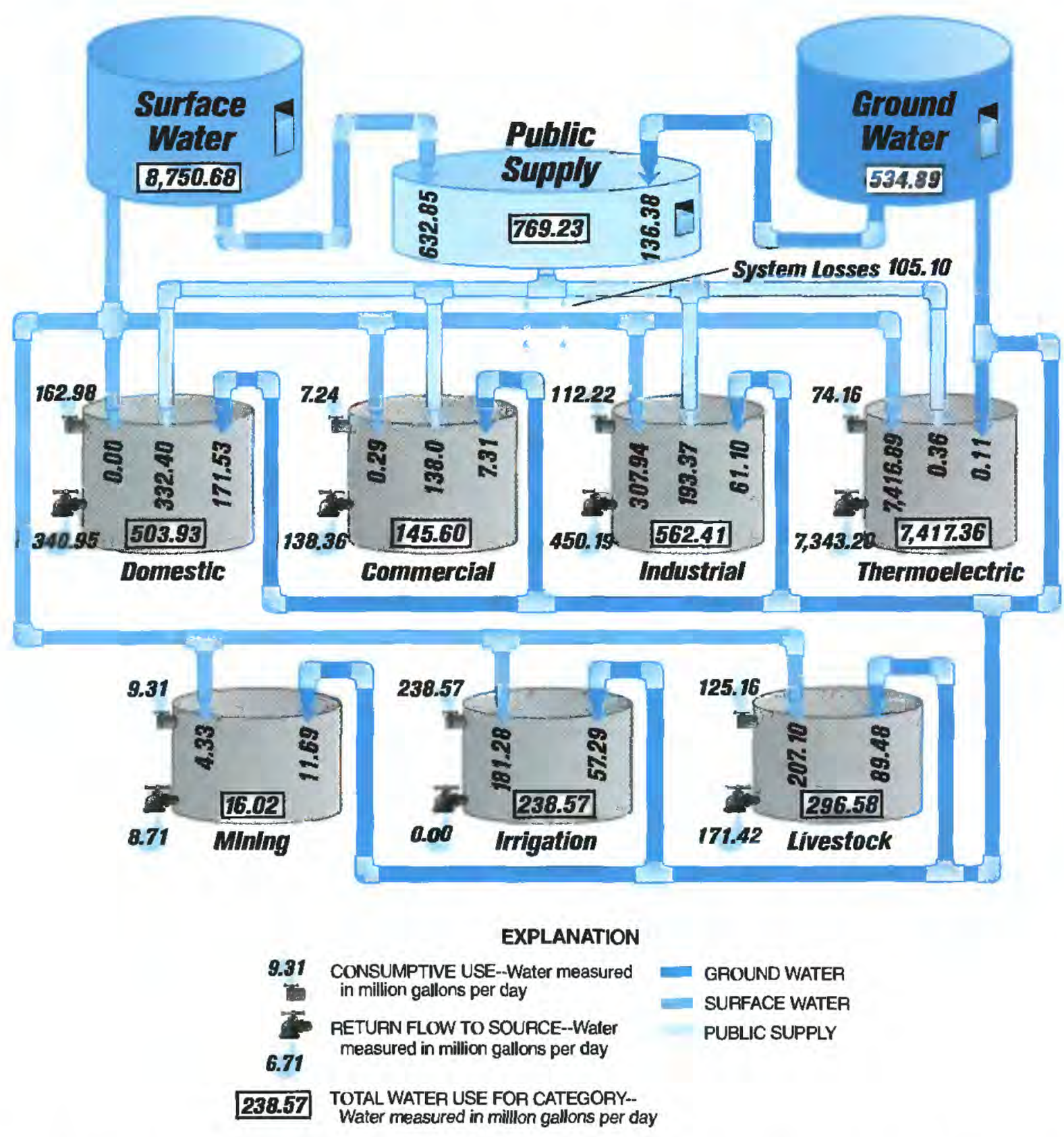

Figure 2. Source, use, and disposition of an estimated 9,286 million gallons per day of fresh and saline water withdrawals in North Carolina, 1995. 
for thermoelectric-power generation. Of the remaining categories, public supply accounted for the largest amount of water withdrawn in North Carolina, representing 8 percent, or about $769 \mathrm{Mgal} / \mathrm{d}$, of total water withdrawals (tables 2 and 3; fig. 3). A summary of water-use estimates, by category and water source, for each county in North Carolina is shown in the appendix of this report.

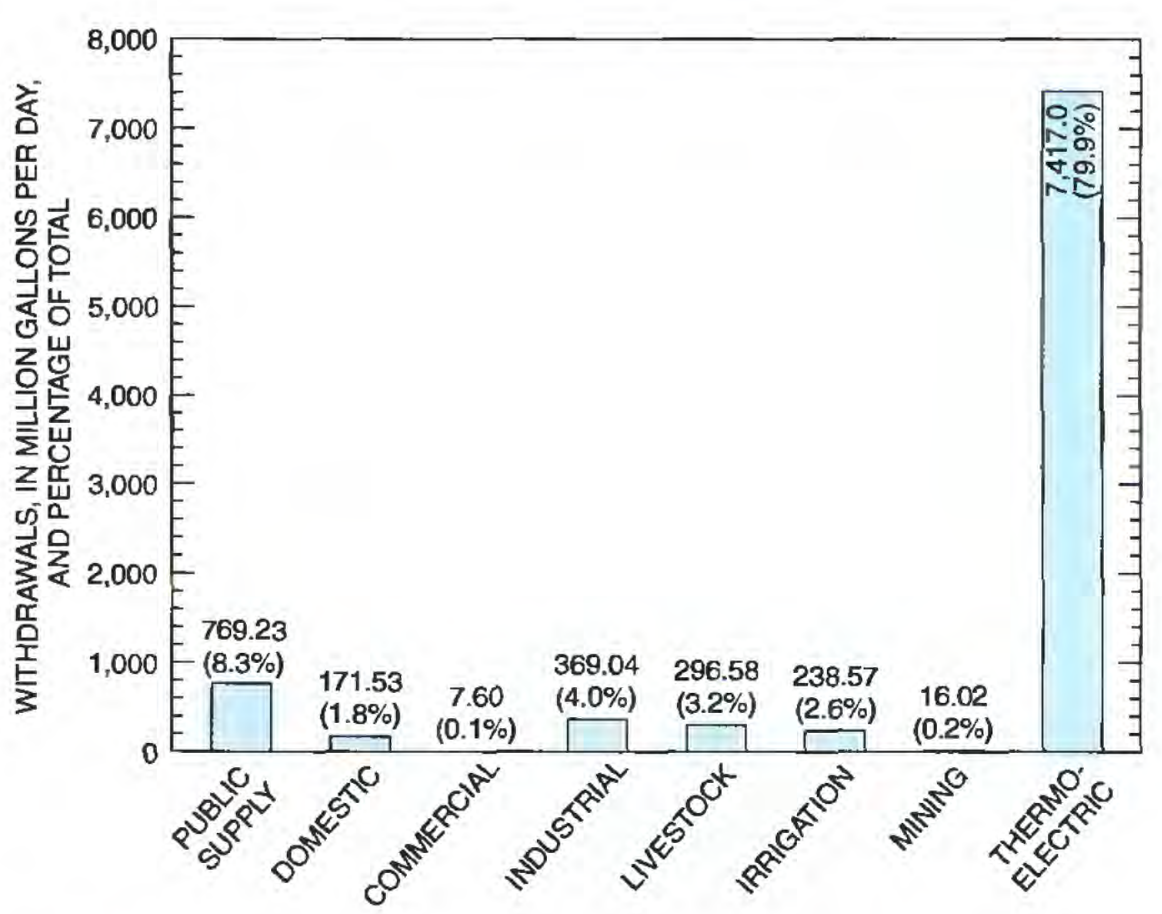

Figure 3. Water withdrawals, by water-use category, in North Carolina, 1995.

Surface water was the source of 94 percent, or 8,751 Mgal/d, of withdrawals in North Carolina in 1995 (table 4; fig. 4); thermoelectric-power generation represented 85 percent of surface-water withdrawals. Public supply was the second largest category of surface-water use, comprising 7 percent of all surfacewater withdrawals (fig. 4).

Six percent of water withdrawn in 1995 was from ground water (table 4; fig. 4). The largest ground-water withdrawals occur primarily in the coastal counties of North Carolina. Of the $535 \mathrm{Mgal} / \mathrm{d}, 26$ percent was withdrawn by public suppliers and 32 percent by domestic users. Agricultural withdrawals (livestock, animal specialties, and irrigation) accounted for 27 percent of ground-water withdrawals, industry and mining accounted for 14 percent (table 2).

For the purpose of this report, instream water use is limited to the use of water for the generation of hydroelectric power. Instream water use is the use of water, but not the withdrawal from a surface-water source. Other examples of instream water use include fish propagation, navigation, and recreation. Because the generation of hydroelectric power requires significant quantities of water that are directed through turbines, this water use is affected by water availability and therefore should be considered in the planning and management of water resources.

\section{Offstream Water Use by Category and Source}

The water-use categories of public supply, domestic, commercial, industrial, mining, livestock, irrigation, and thermoelectric-power generation are
A.

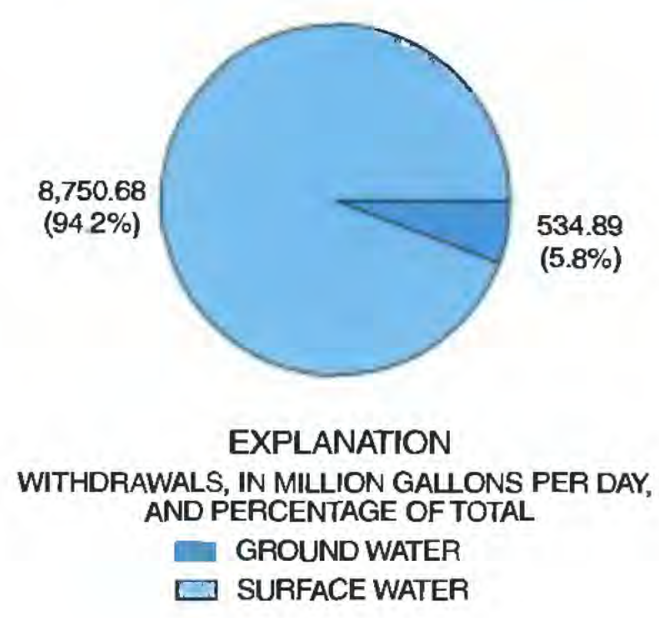

B.

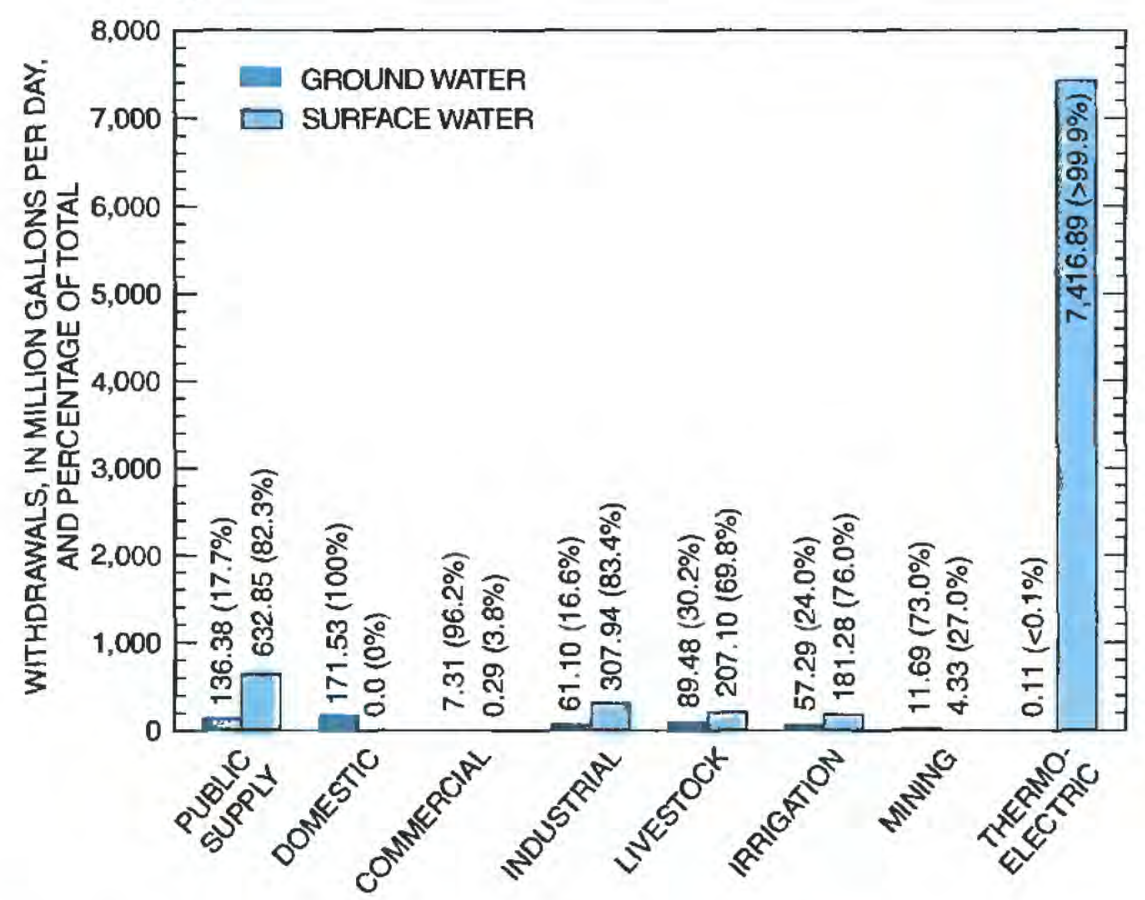

Figure 4. Water withdrawals, by (A) source and (B) water-use category and source, in North Carolina, 1995. 


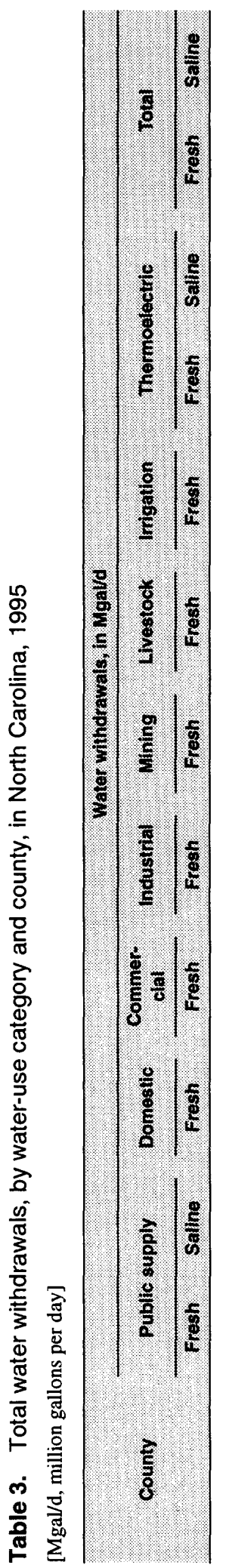

880808 $8880 \% 0 \%$

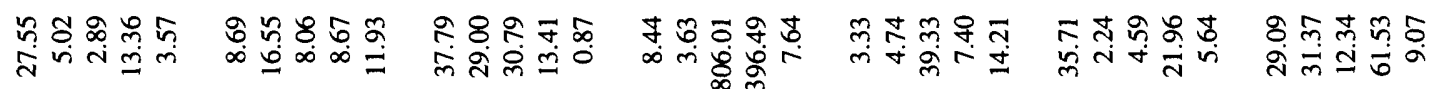

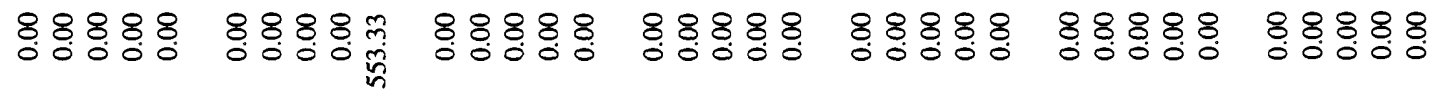

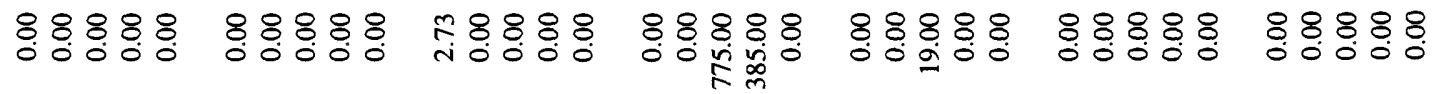

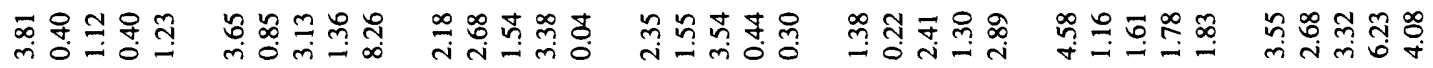

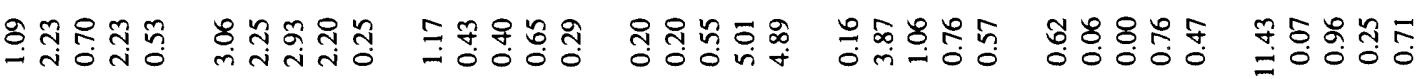

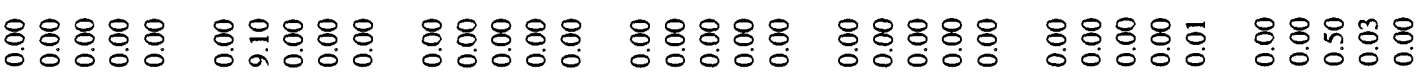

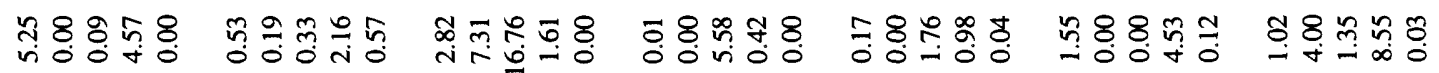

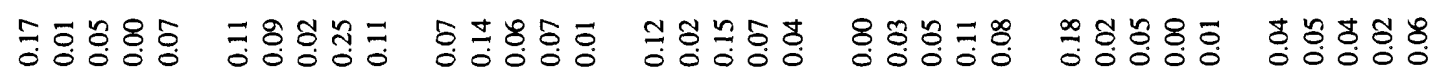

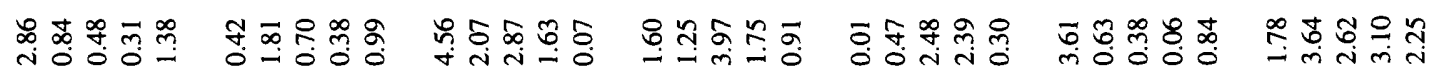

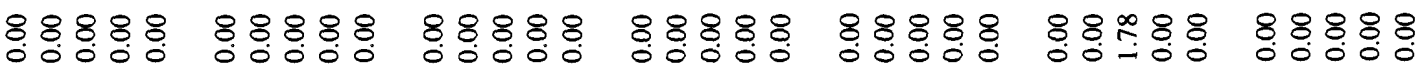

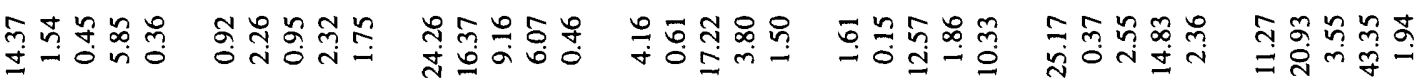

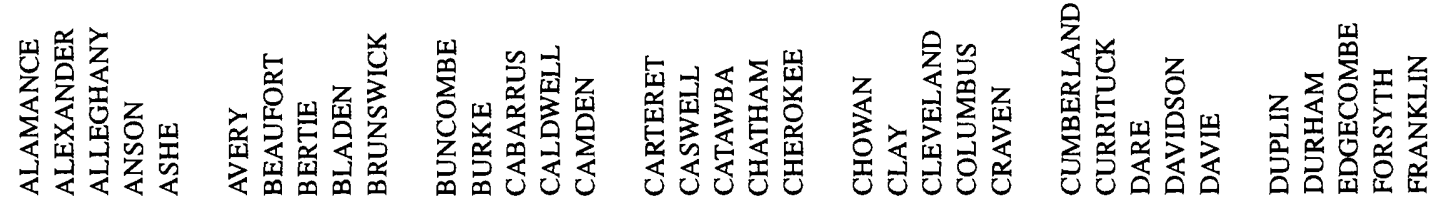

Estimated Water Use $\quad 7$ 


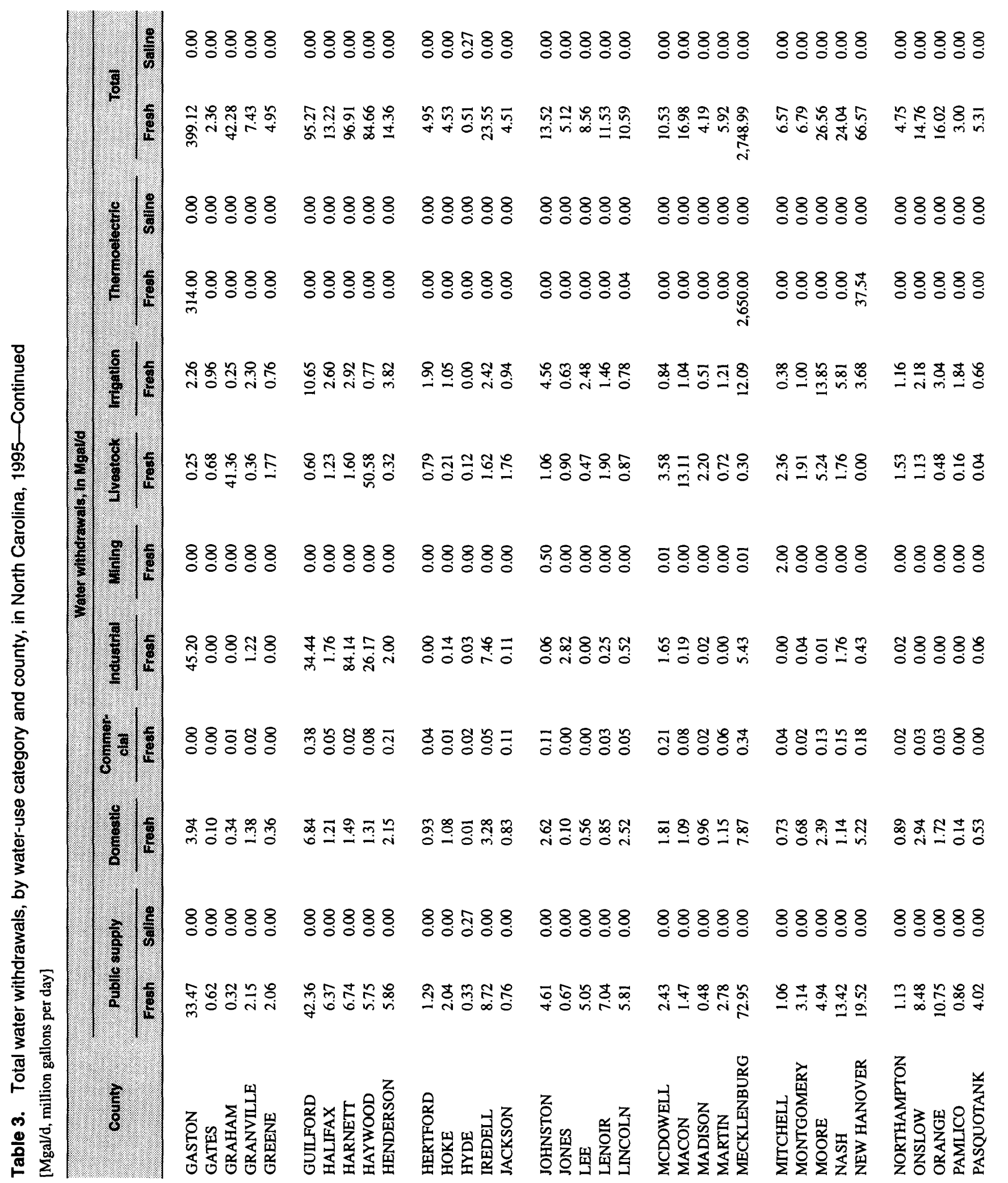

8 Estimated Water Use, by County, in North Carolina, 1995 


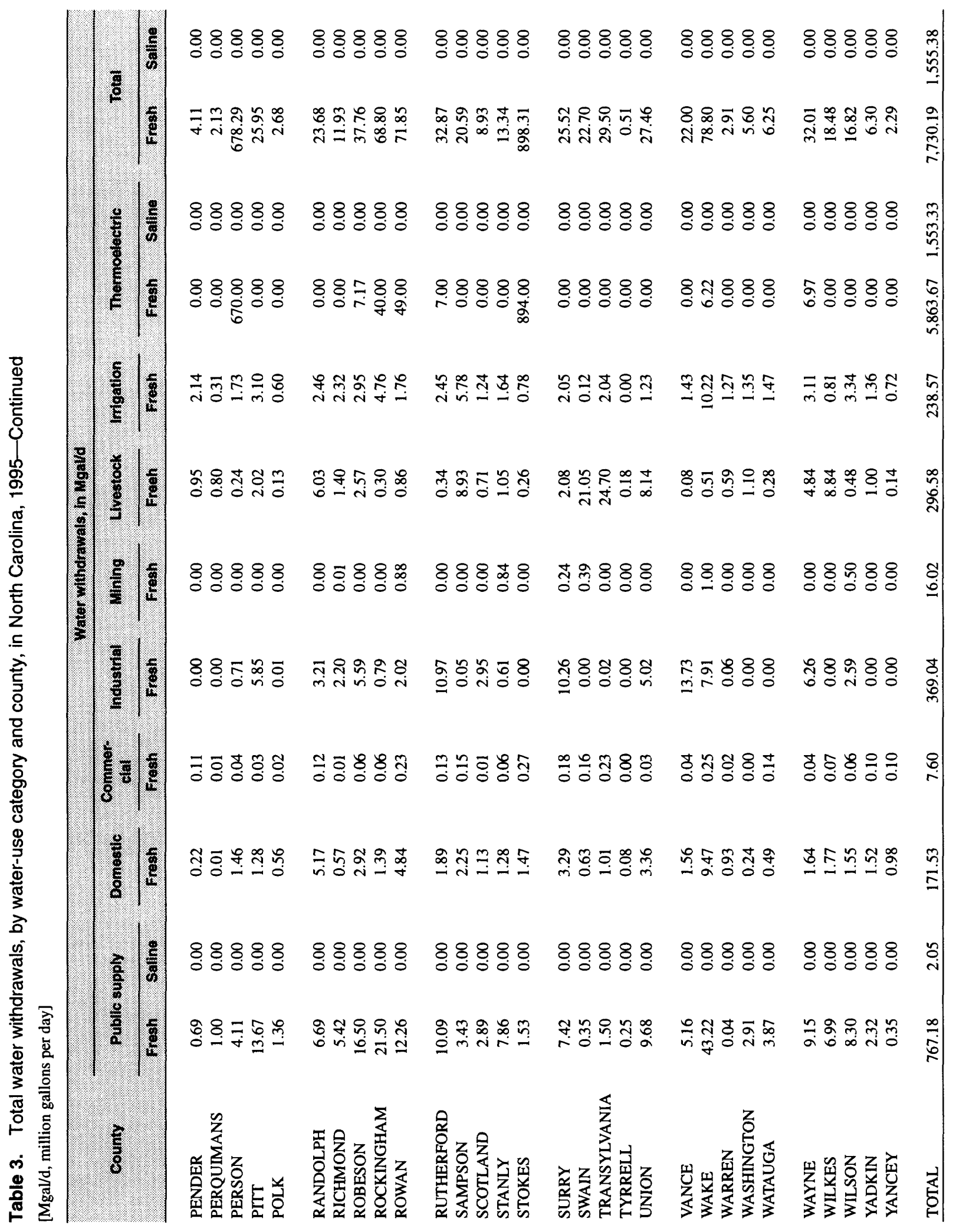




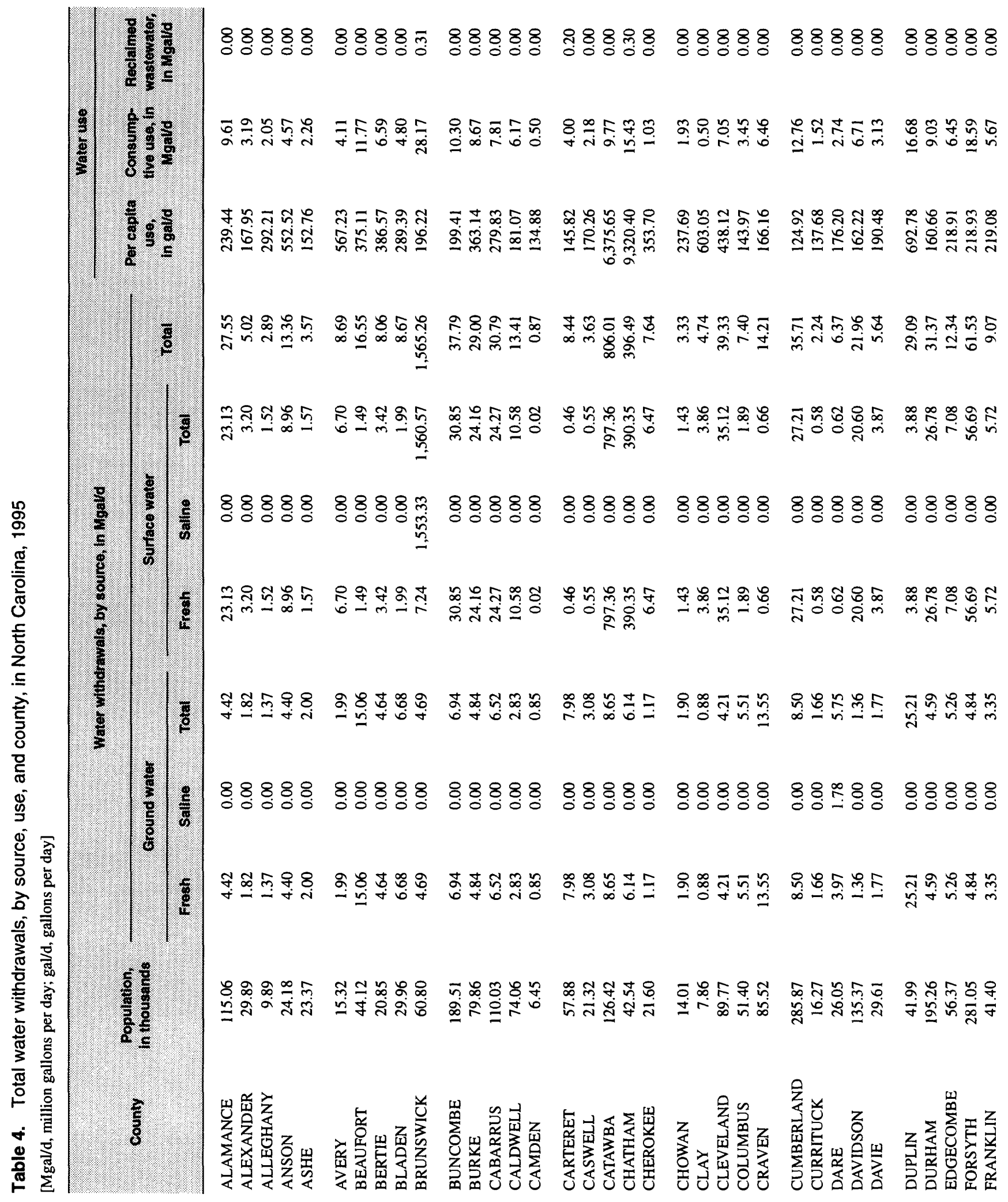




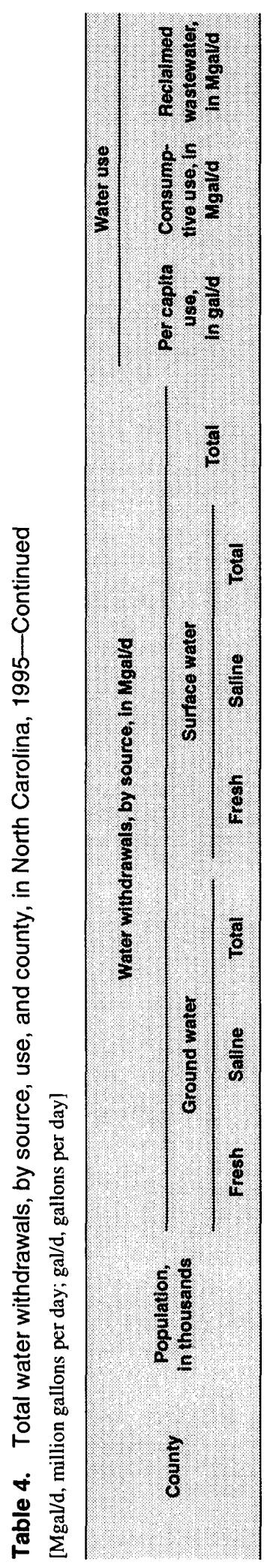

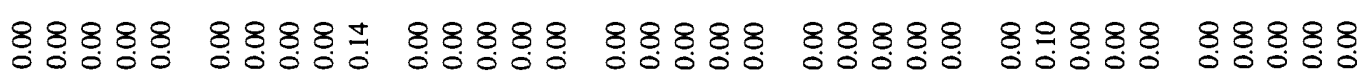

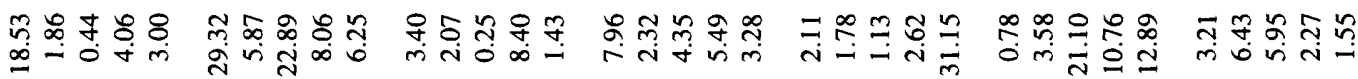

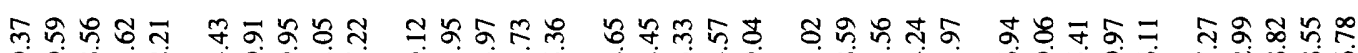

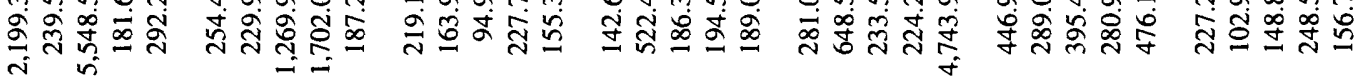

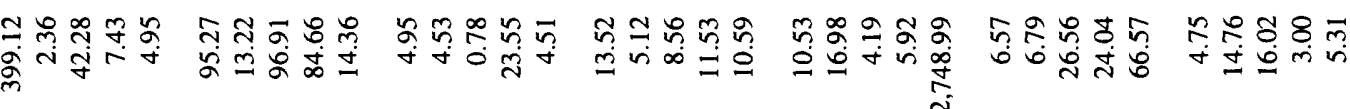

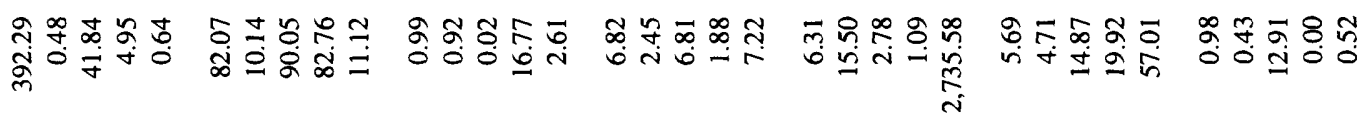

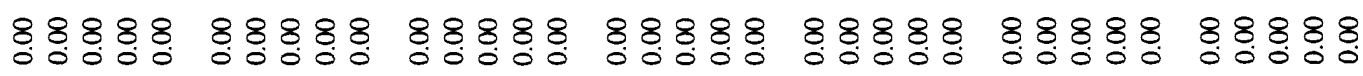

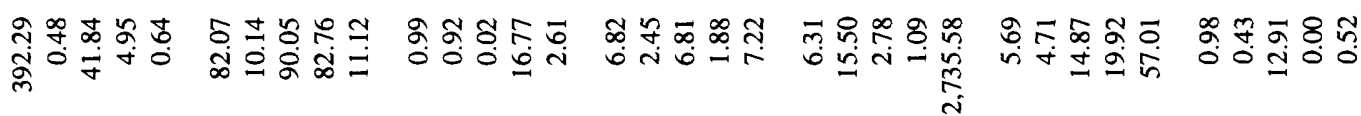

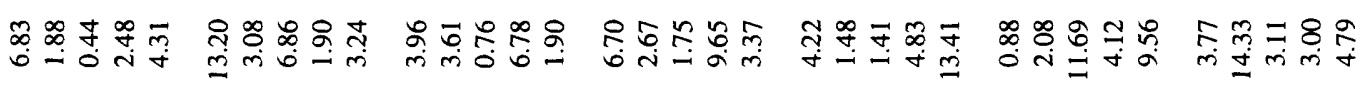

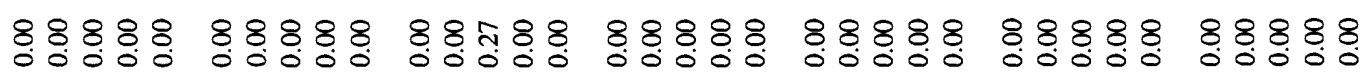

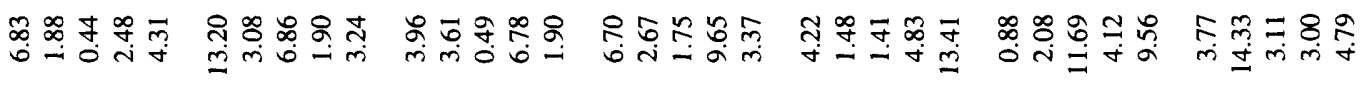

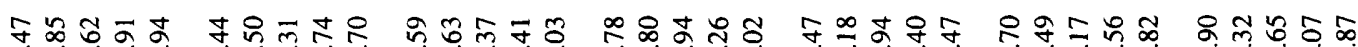

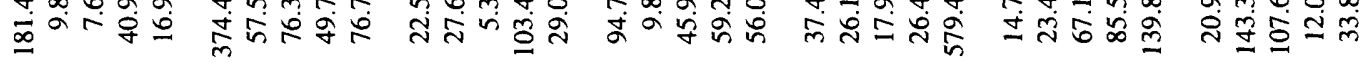

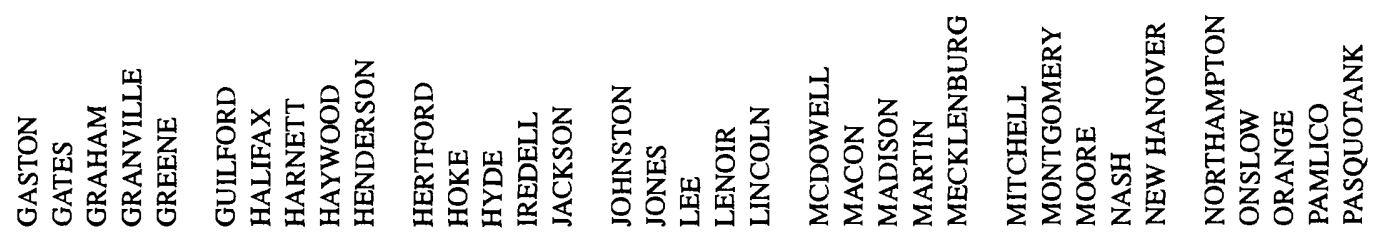




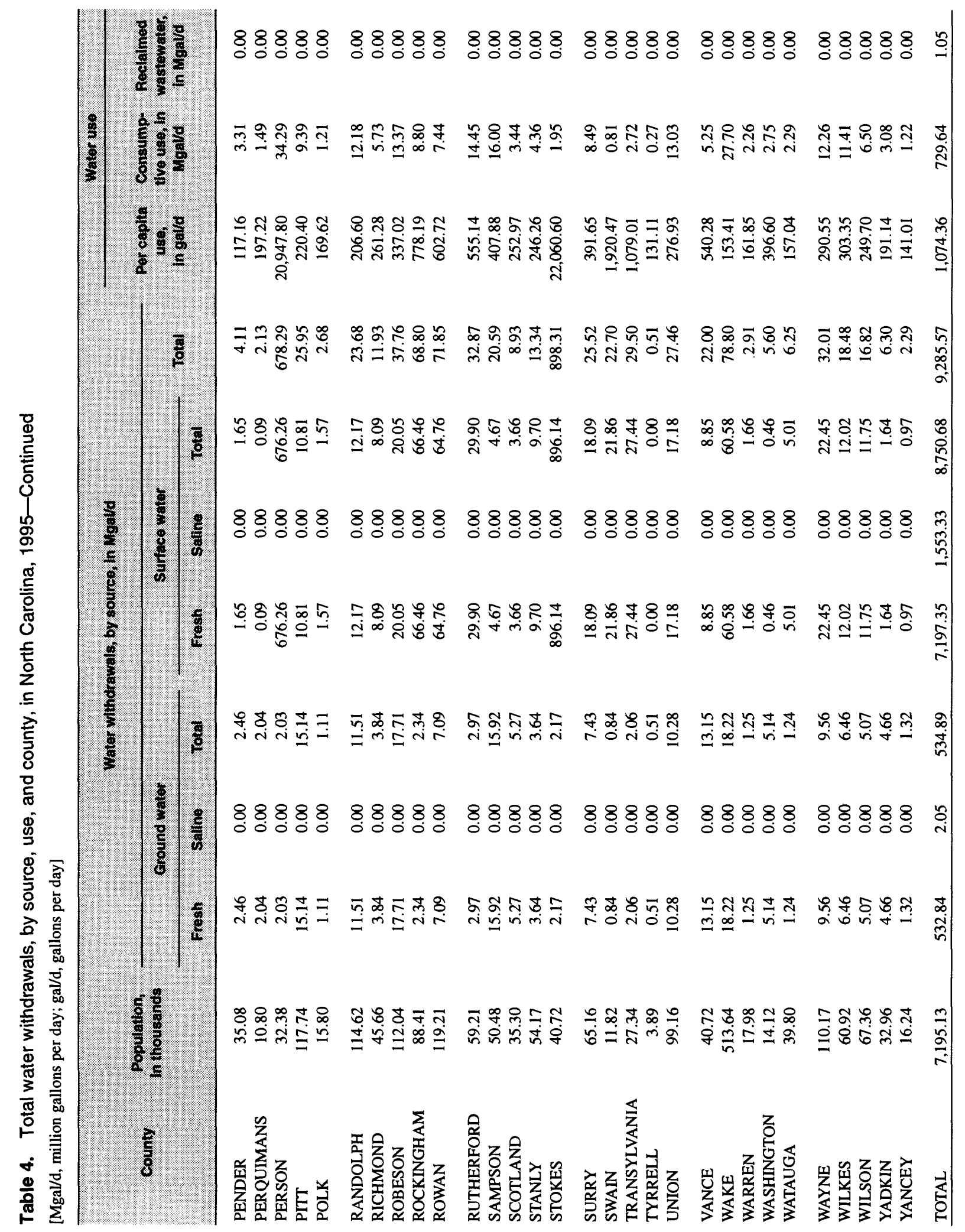


summarized by county in table 3 and by source of water in figure 5. Counties with the largest withdrawals are those where thermoelectric-power generation facilities are located. Brunswick and Mecklenburg Counties each withdrew more than 1,500 Mgal/d. Three other counties (Catawba, Person, and Stokes) each withdrew more than $500 \mathrm{Mgal} / \mathrm{d}$ (table 3). Most of the water withdrawn was in conjunction with power generation.

Consumptive use is defined as "that part of water withdrawn that is evaporated, transpired, incorporated into products or crops, consumed by humans or livestock, or otherwise removed from the immediate water environment" and, therefore, not returned to the source of withdrawal (Solley and others, 1993). Person
County has the highest consumptive use in the State with about $34 \mathrm{Mgal} / \mathrm{d}$ (table 4), because of the high consumptive-use rate for thermoelectric-power generation. Excluding thermoelectric, Mecklenburg County has the highest consumptive-use rate in the State with $31 \mathrm{Mgal} / \mathrm{d}$. Excluding thermoelectric, other counties with consumptive use amounts greater than $15 \mathrm{Mgal} / \mathrm{d}$ include Duplin, Forsyth, Gaston, Guilford, Harnett, Moore, Sampson, and Wake. These counties may have large populations served by public suppliers, large domestic, agricultural water withdrawal rates, or a combination of all. The impact of high consumptive use is that the water is no longer available to other users within or downstream from the county.

\section{A. Total withdrawals}

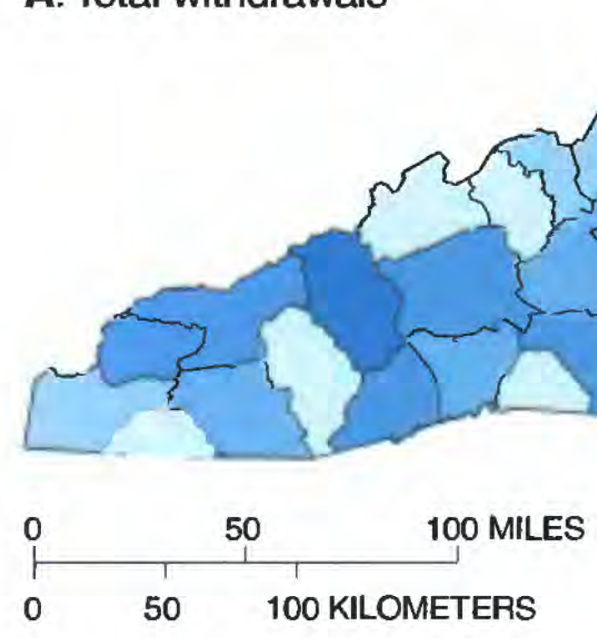

B. Surface-water withdrawals
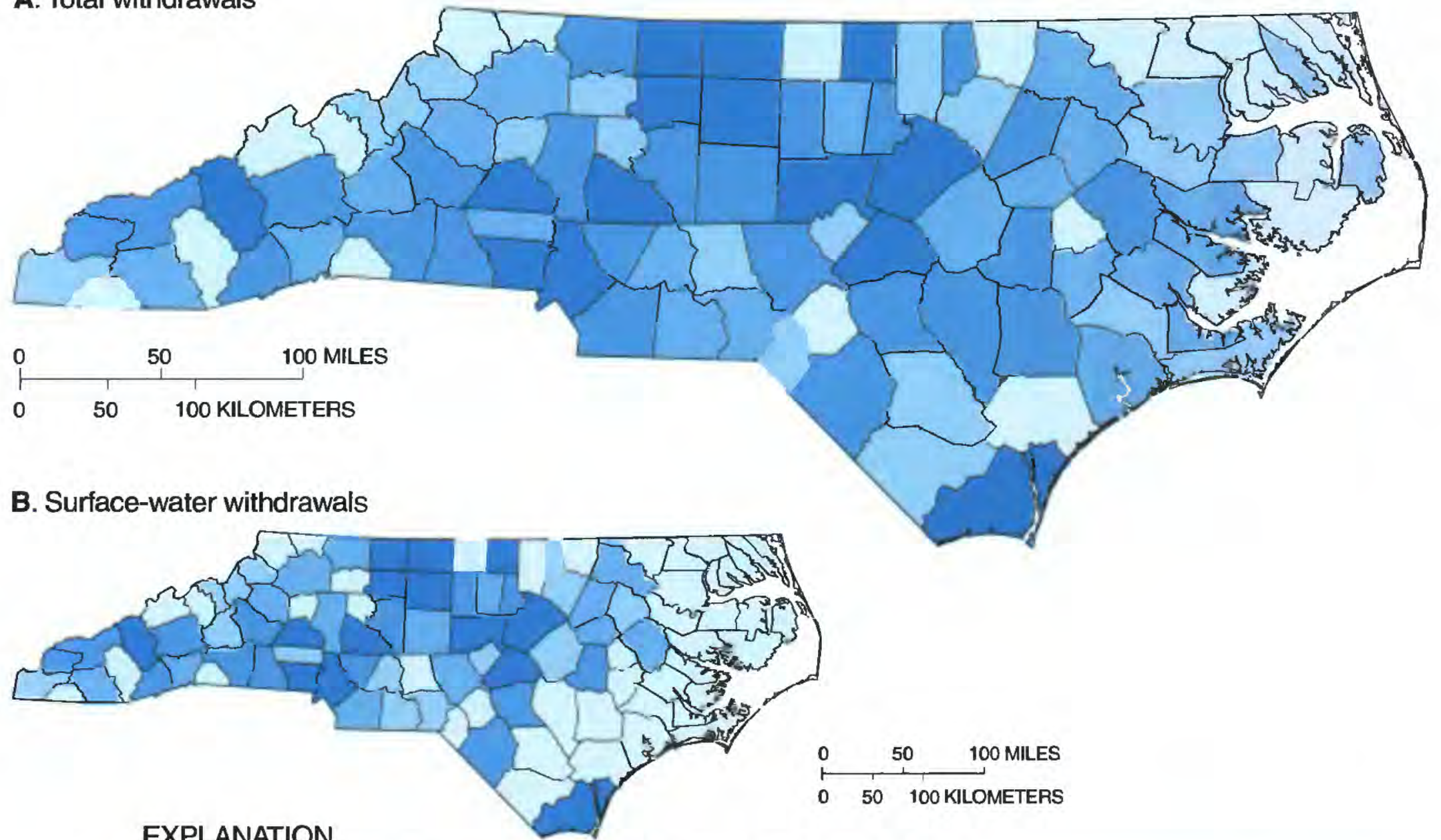

EXPLANATION

WITHDRAWALS, IN MILLION

GALLONS PER DAY

\subsection{OR LESS}

$5.00-9.99$

$10.00-19.99$

$20.00-49.99$

50 OR MORE

\section{Ground-water withdrawals}

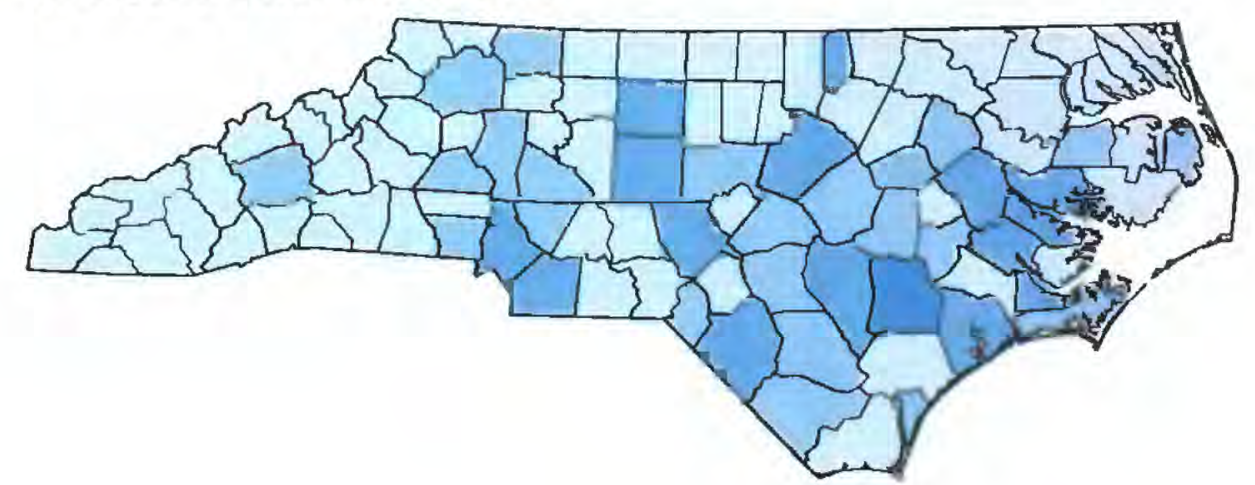

Figure 5. Water withdrawals, by county, in North Carolina, 1995. (Data from the U.S. Geological Survey National Data Storage and Retrieval System.) 


\section{Public-Supply Water Use}

The population of North Carolina was about 7.2 million in 1995 (table 4), an increase of almost 8 percent since 1990 (U.S. Bureau of the Census, 1996). About 66 percent, or 4.7 million people, were served by a public-supply system. Counties with the majority of their population residing in urban areas tend to have a greater number of the population served by public suppliers. Mecklenburg County (Charlotte) had the highest population $(579,470)$ in the State, with 467,070 people served by public supply. Other more populated areas of the State (in order of highest population) include Wake, Guilford, Cumberland, Forsyth, and Durham Counties (table 4). A large percentage of the population in these counties are served by public-supply systems (table 5 ; fig. 6).

Public-supply systems withdraw, treat, and deliver water to customers. An estimated $769 \mathrm{Mgal} / \mathrm{d}$ of water was withdrawn for public supply in 1995. This number is 8 percent of all water withdrawn in the State. Withdrawals for this category are primarily from surface water which accounted for 82 percent of the

A. Total population
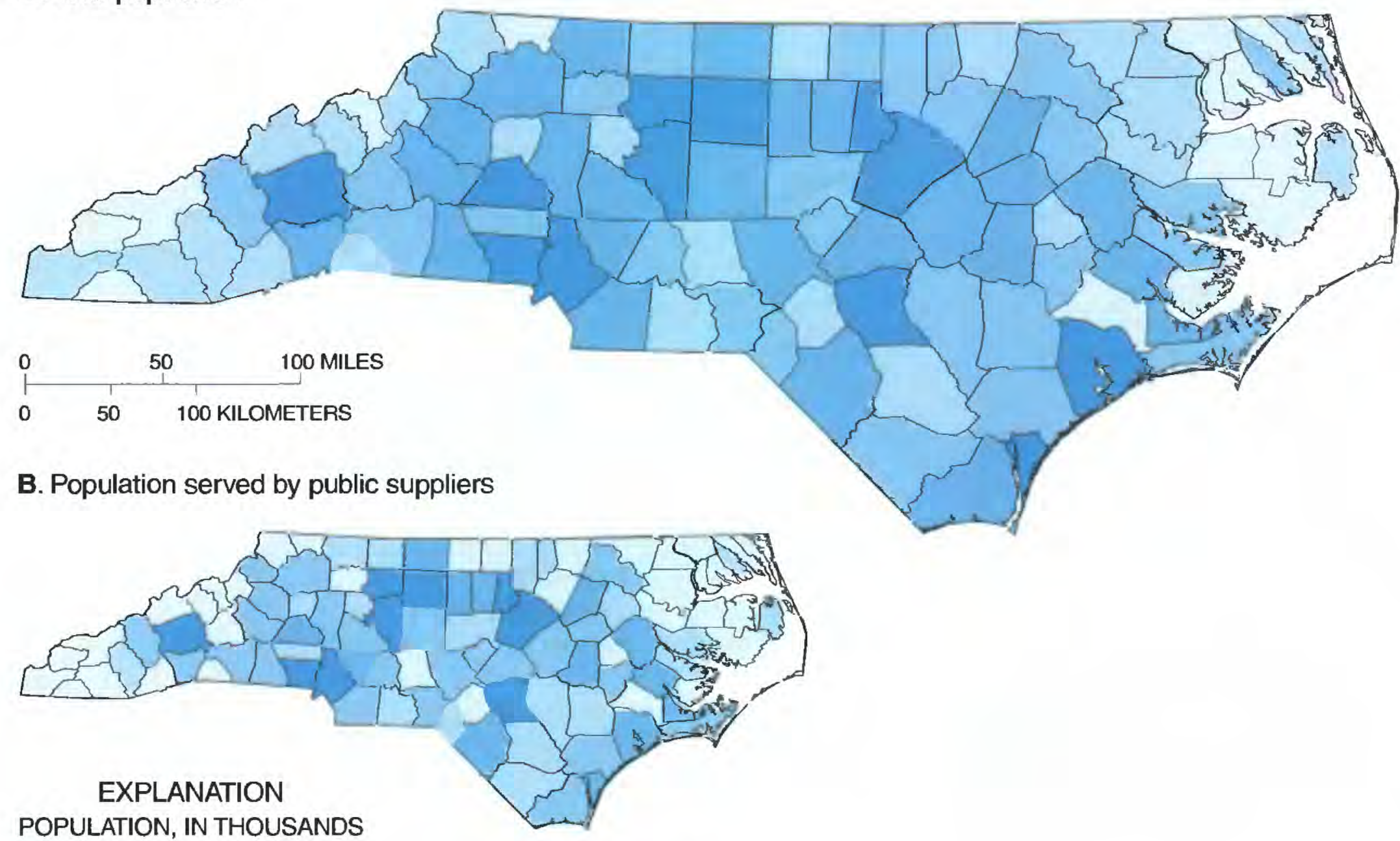

POPULATION, IN THOUSANDS
14.99 OR LESS
$15.00-29.99$
$30.00-59.99$
$60.00-119.99$
120 OR MORE

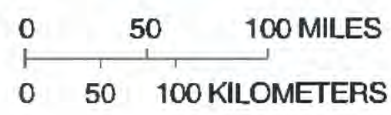

Figure 6. (A) Total population and (B) population served by public suppliers, by county, in North Carolina, 1995. (Data from the U.S. Geological Survey National Data Storage and Retrieval System.) 


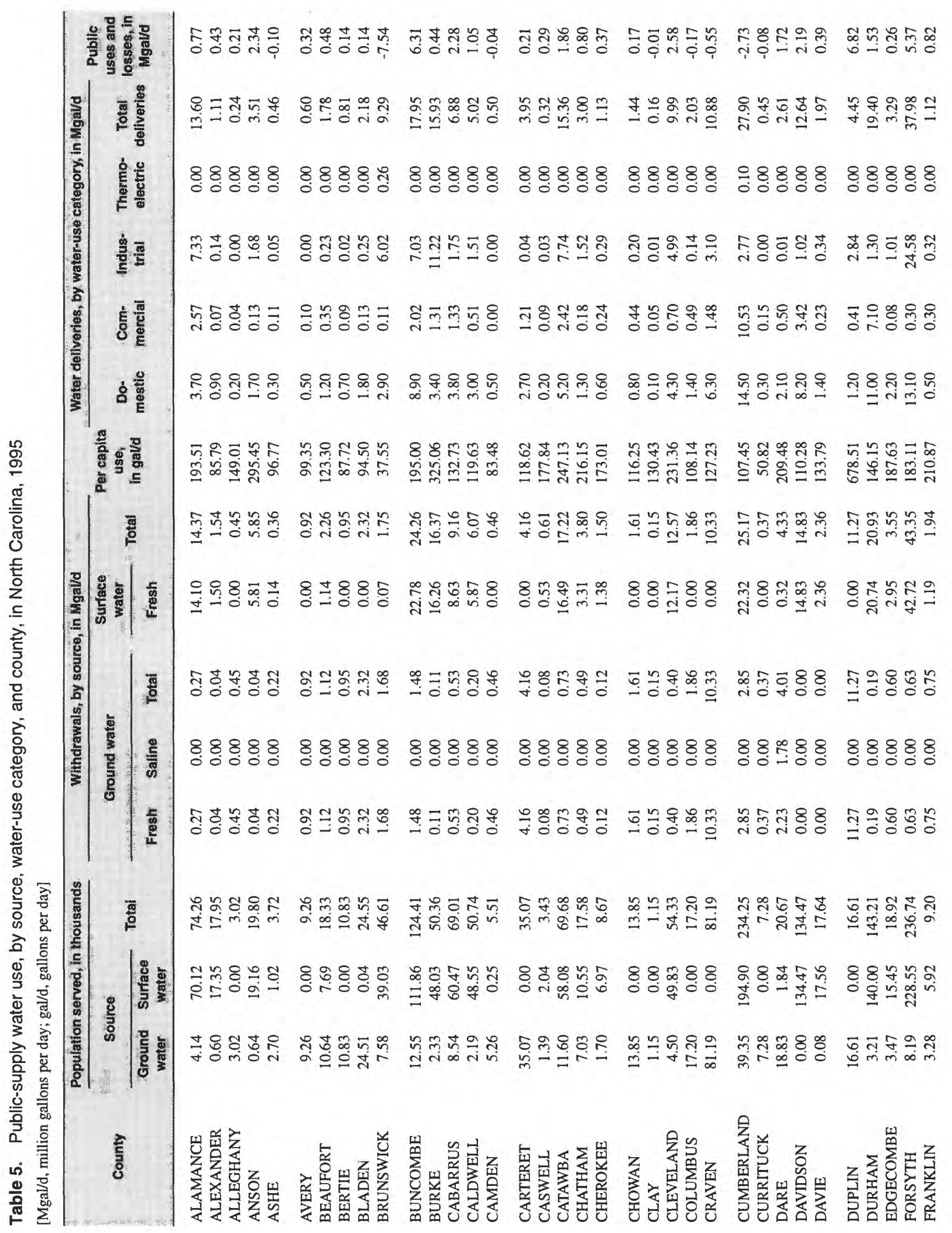




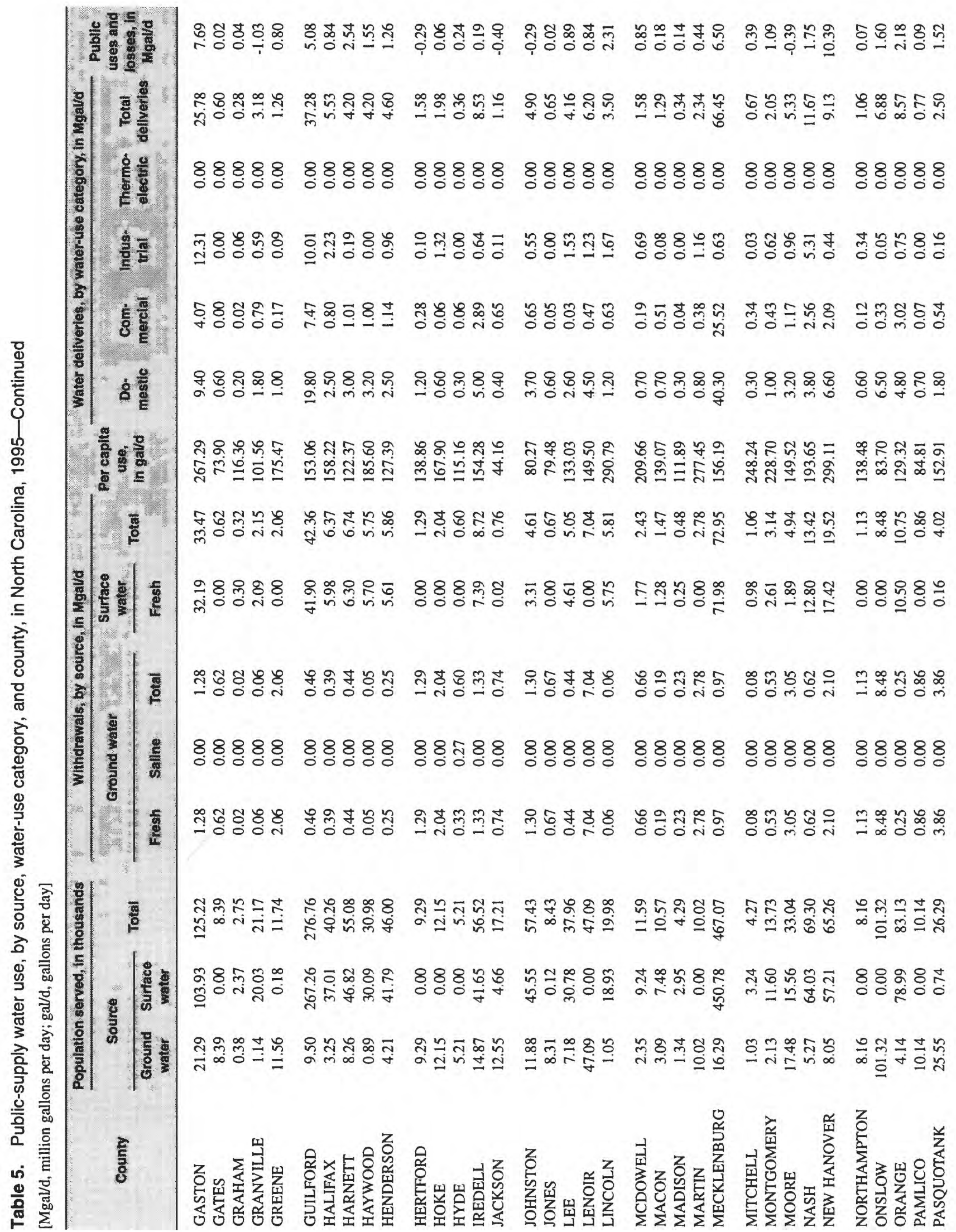




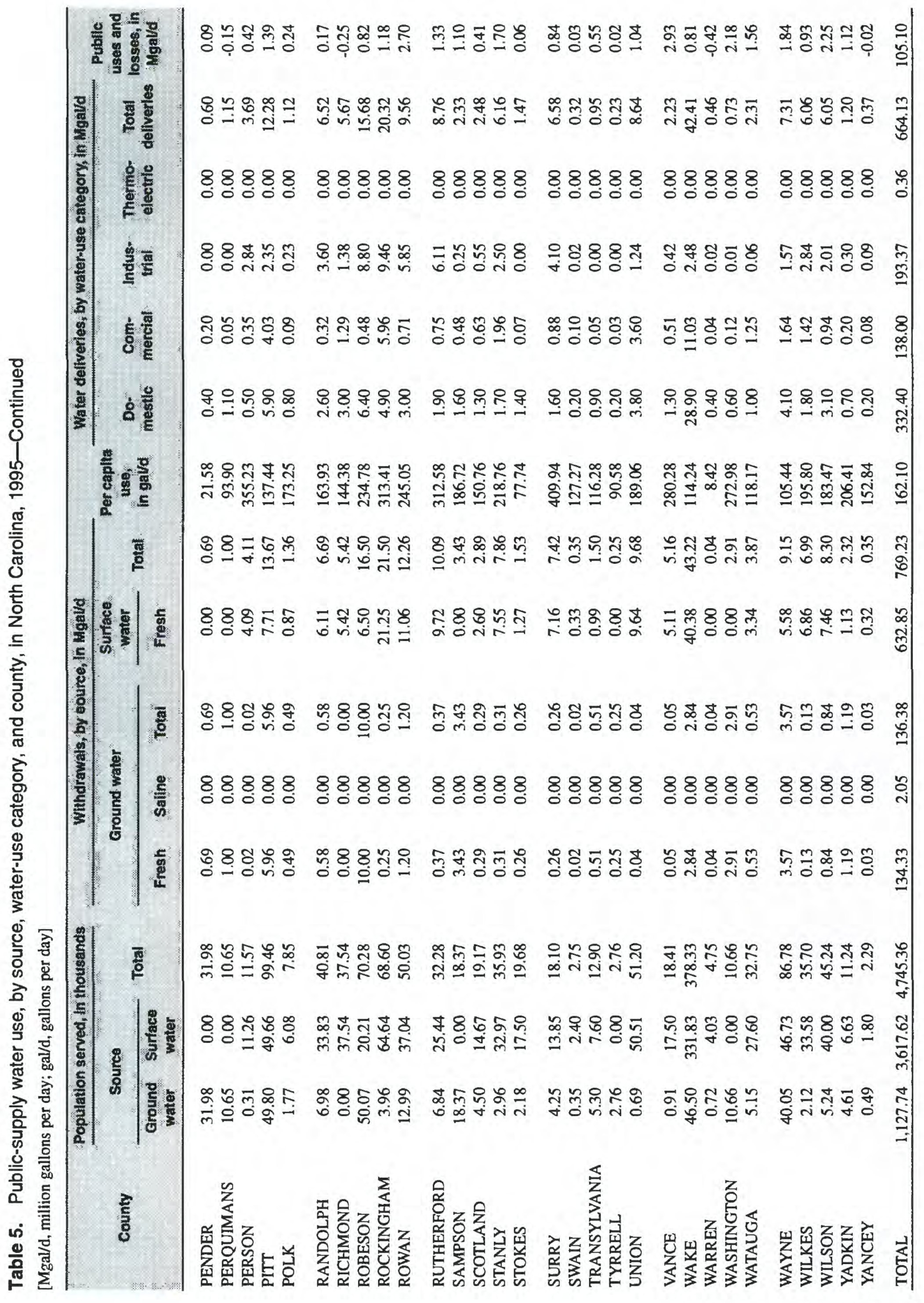


public-supply water (table 5; fig. 7). About $2 \mathrm{Mgal} / \mathrm{d}$ of saline water was withdrawn from aquifers in Dare and Hyde Counties and treated for public-supply use.

Public-supply systems delivered water for domestic, commercial, industrial, thermoelectric power, and for other public uses (such as firefighting, street cleaning, and recreation). Deliveries to the domestic sector account for 43 percent of the publicsupply withdrawals (fig. 7 ). The remaining 57 percent includes deliveries to the other sectors, losses resulting from the transfer of water outside of the county or State, and losses associated with leaks within the distribution systems (table 5). The "public use and losses" column in table 5 was computed by subtracting the total deliveries to domestic, commercial, industrial, and thermoelectric water users from the total water withdrawals.

In the last few decades many municipalities have expanded their service areas to keep up with increasing population, and many previously self-supplied rural areas have been connected to public or community water systems. Mecklenburg County had the largest rate of withdrawal by public suppliers (nearly 10 percent of total public-supply withdrawals, or $73 \mathrm{Mgal} / \mathrm{d}$ ) (table 5). Population served by public suppliers in Mecklenburg County accounted for 10 percent of all population served by public suppliers in the State. Wake, Forsyth, and Guilford Counties are the other large users of publicly supplied water (more than $40 \mathrm{Mgal} / \mathrm{d}$ ) in the State (table 5; fig. 8). These counties account for 19 percent of all population served, and 18 percent of all the water delivered, by public suppliers in the State (table 5). Concentrated industrial and commercial activities also contribute to the large public-supply deliveries in these counties.

\section{Domestic Water Use}

In 1995, water use (withdrawals plus deliveries) for domestic purposes was $504 \mathrm{Mgal} / \mathrm{d}$ and represents 5 percent of all the water withdrawn in North Carolina (table 6). Nearly 2.5 million people in North Carolina rely on private wells or springs for their household
A.

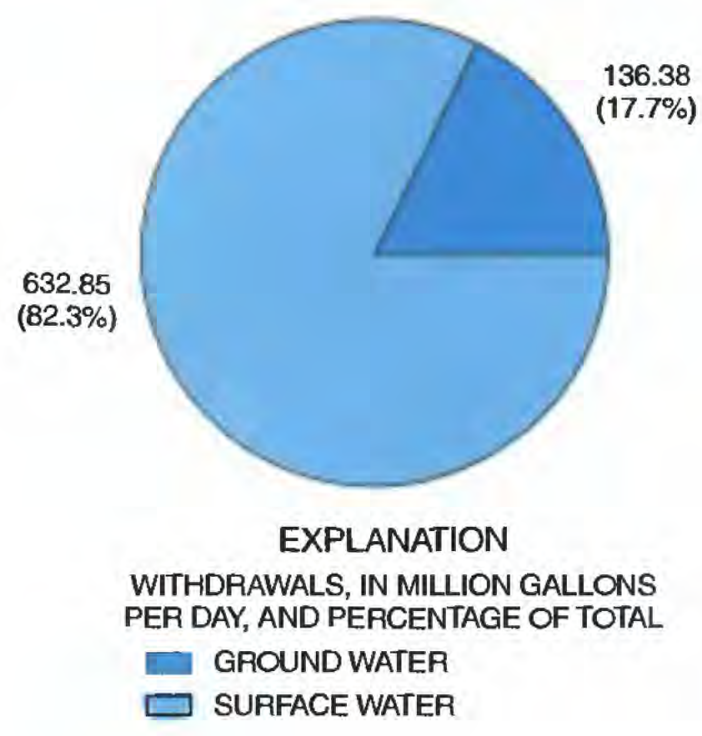

B.

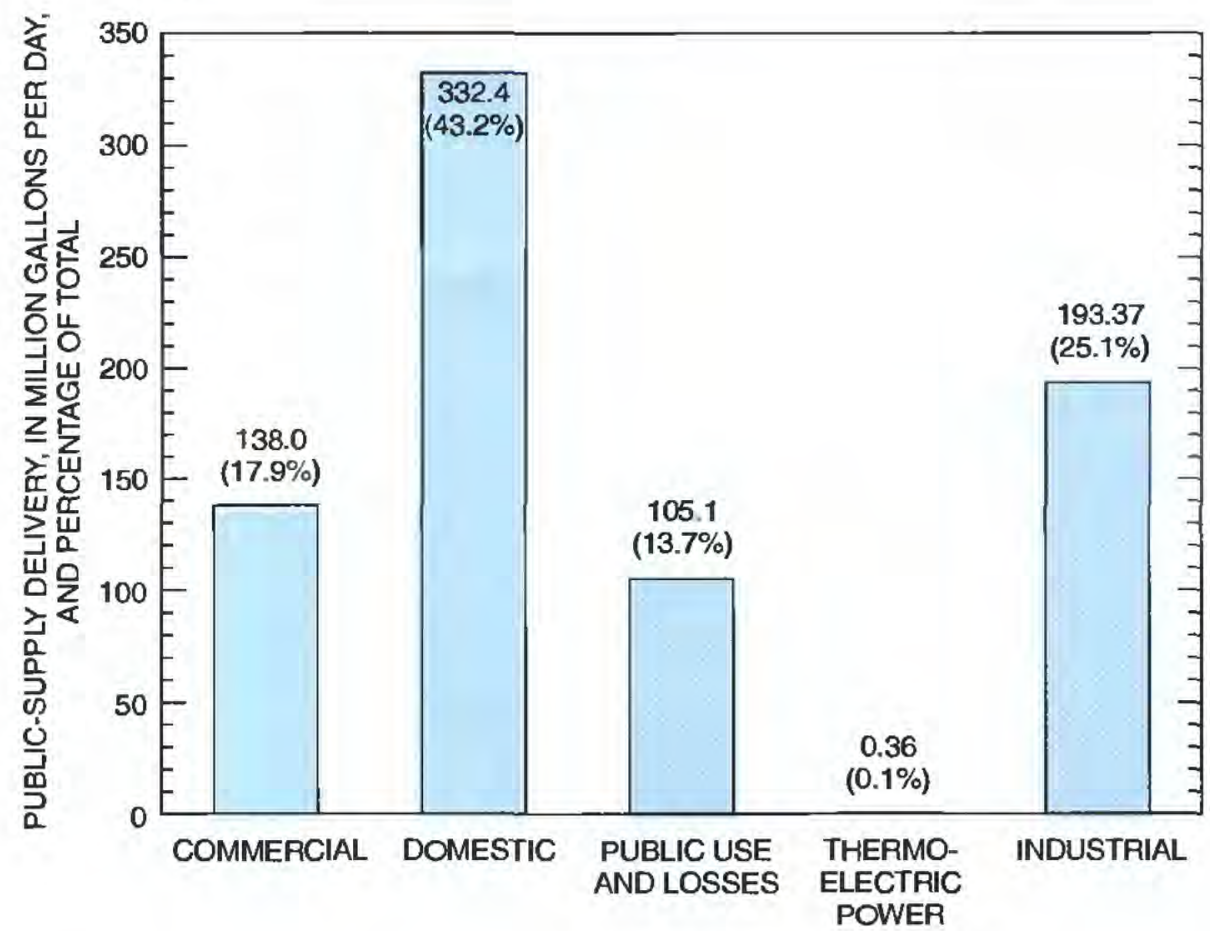

Figure 7. (A) Sources of public-supply withdrawals and (B) public-supply deliveries, by water-use category, in North Carolina, 1995. 

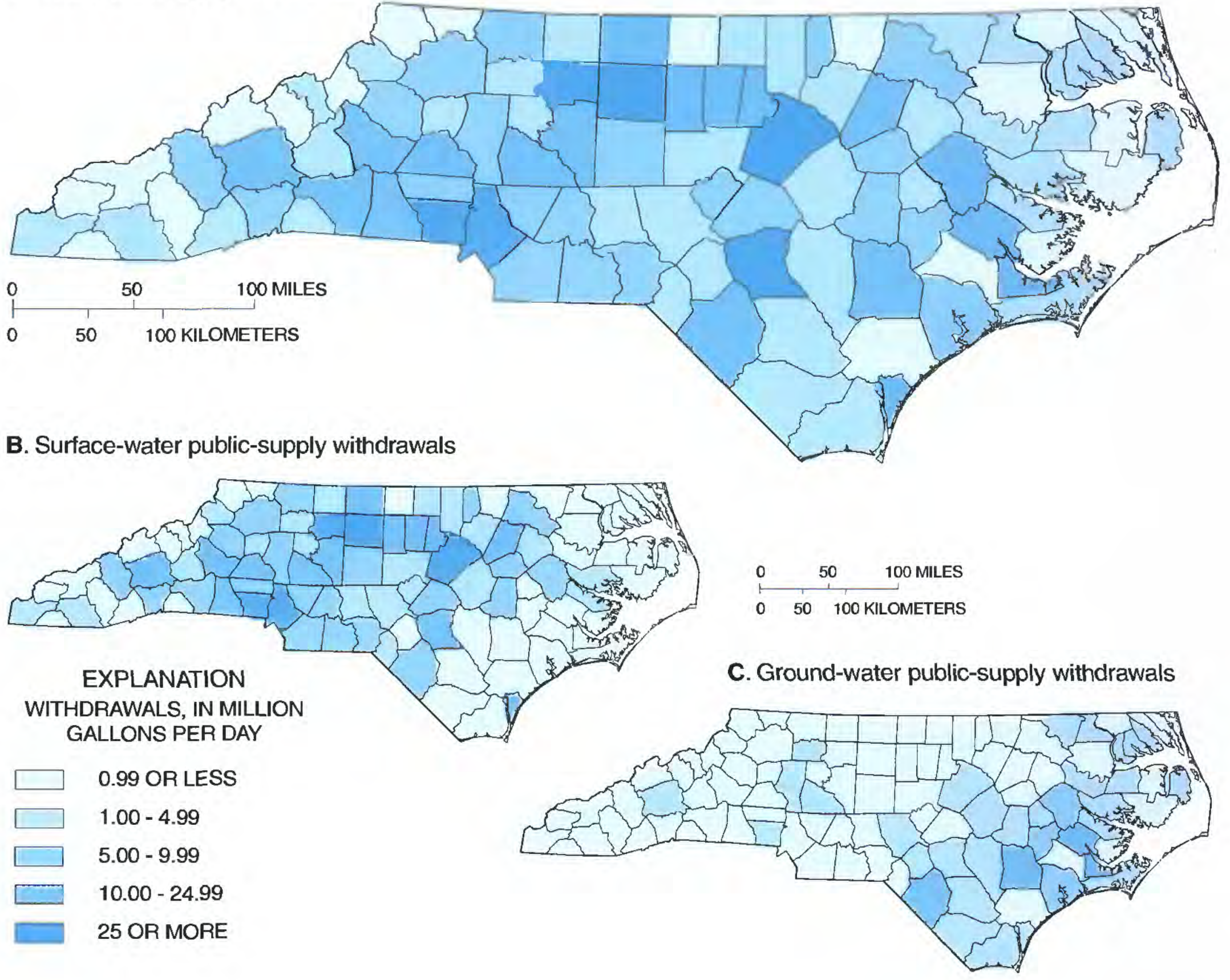

Figure 8. Public-supply withdrawals, by county, in North Carolina, 1995. (Data from the U.S. Geological Survey National Data Storage and Retrieval System.)

water needs (table 6; fig. 9). Withdrawals for domestic use were $172 \mathrm{Mgal} / \mathrm{d}$. Domestic withdrawals were estimated with a per capita use rate of 70 gallons per day (gal/d). Per capita use for people serviced by public suppliers was also about $70 \mathrm{gal} / \mathrm{d}$. Public suppliers delivered $332 \mathrm{Mgal} / \mathrm{d}$ to about 4.7 million users; this accounts for 66 percent of all water used for domestic purposes (table 5 ; fig. 9).

Wake County had the largest self-supplied population in the State with 135,300 persons (table 6; fig 10). Mecklenburg, Guilford, New Hanover, and Randolph Counties also had large populations that were self supplied. Each of these five counties withdrew $5 \mathrm{Mgal} / \mathrm{d}$ or more, and combined, accounted for 20 percent of all domestic withdrawals in 1995 (table 6; fig. 11). Domestic withdrawals were assumed to be entirely from ground water.
More than 75 percent of the people in seven counties relied on private wells and springs in 1995 for their water supply: Yancey, 86 percent; Clay, 85 percent; Ashe and Caswell, 84 percent each; Franklin, 78 percent; Swain, 77 percent; and Madison, 76 percent (table 6; fig. 10). These counties have relatively small populations that are located in rural areas of the State. In contrast, counties with less than 5 percent of the population that relied on private water supplies include Davidson, Chowan, and Perquimans, 1 percent each; Hyde, 3 percent; and Craven, 5 percent. Chowan, Perquimans, Hyde, and Craven Counties are mostly rural areas with small total populations served primarily by public-supply systems. Davidson County has a relatively large population, and several urban areas are served by public-supply systems in Davidson or adjoining counties. 


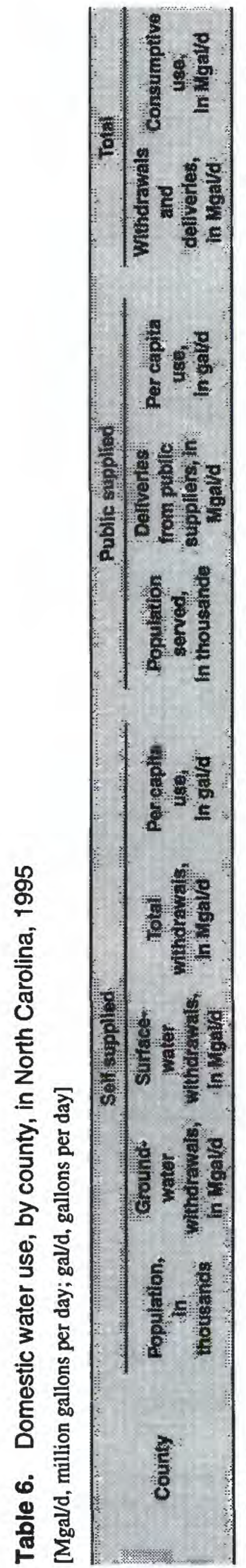

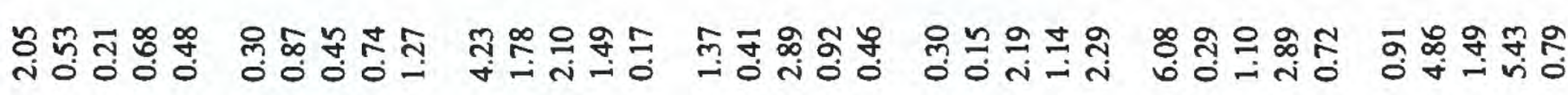

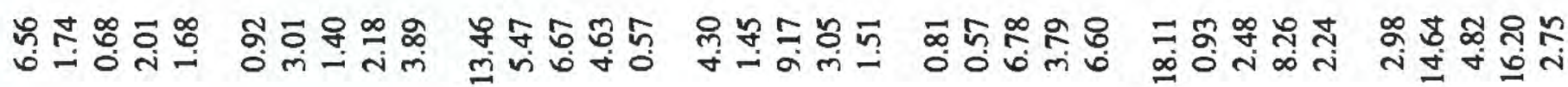

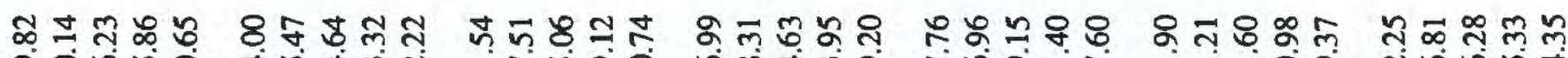

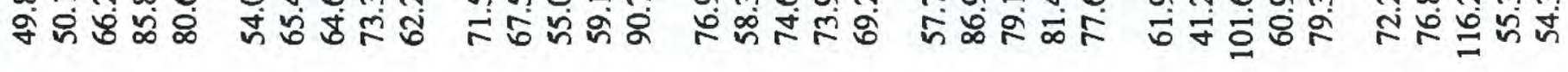

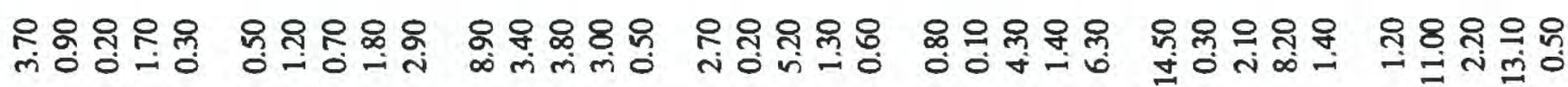

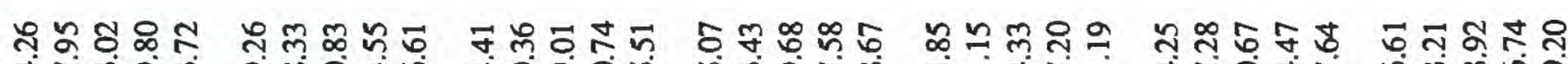

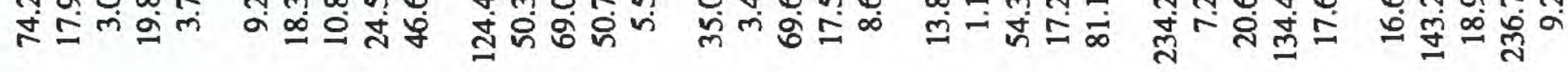

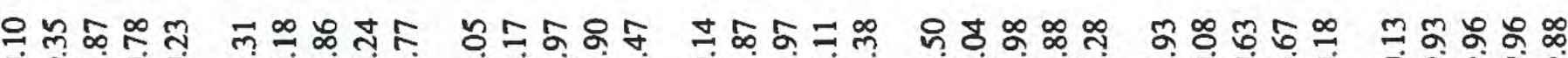

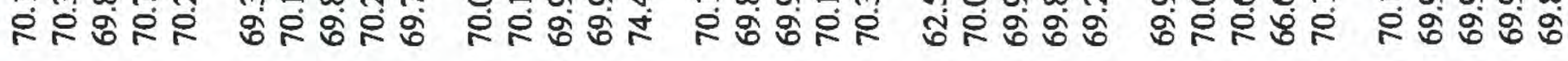

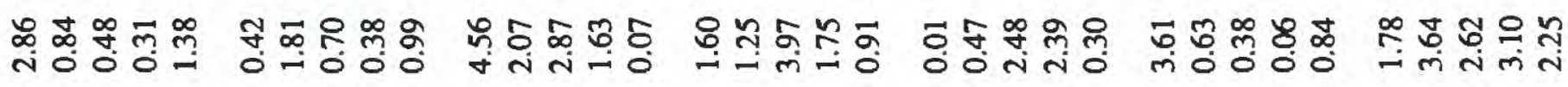

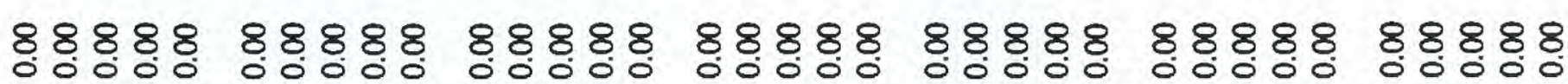

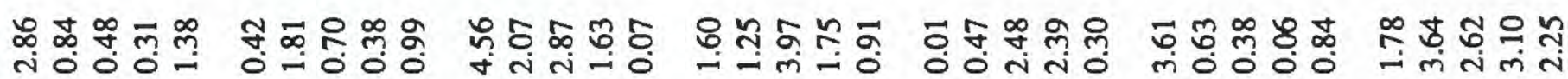

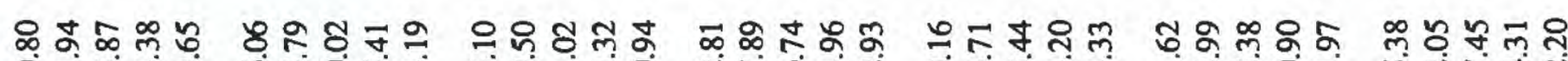

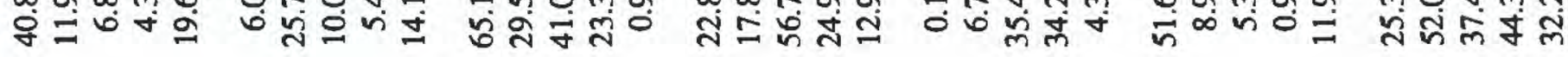

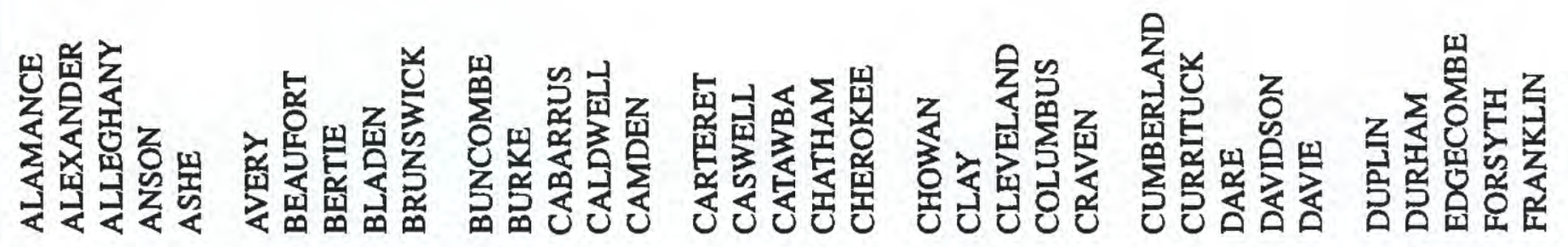




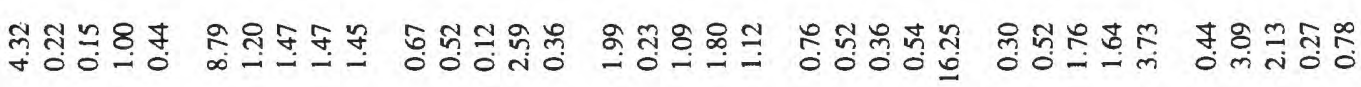

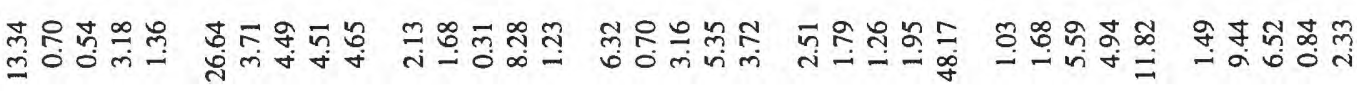

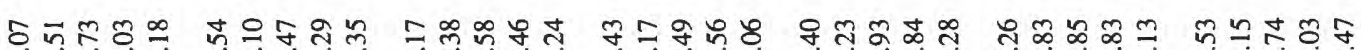
लतㅛ

웅융영 \& 엄

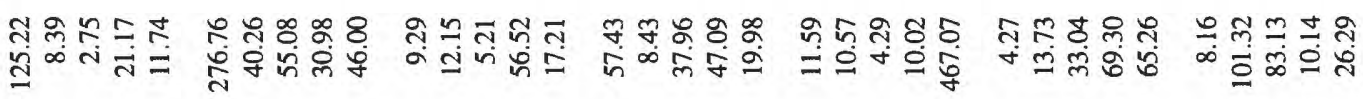

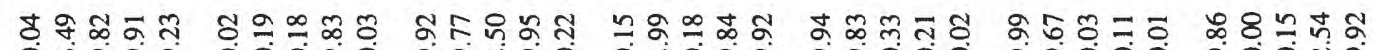

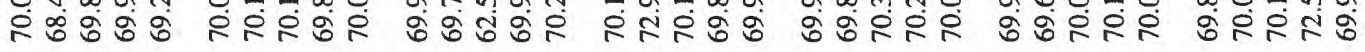

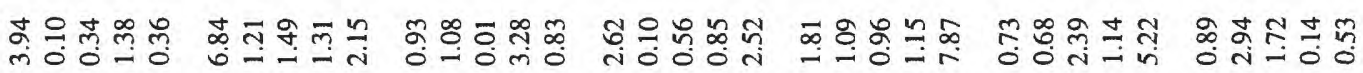

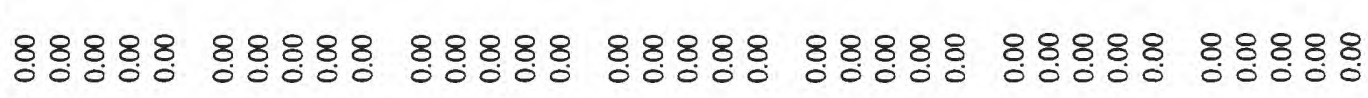

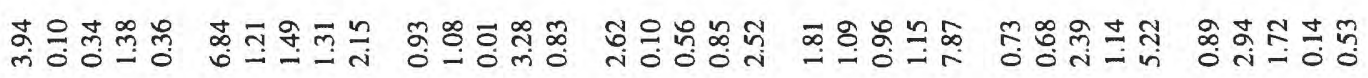

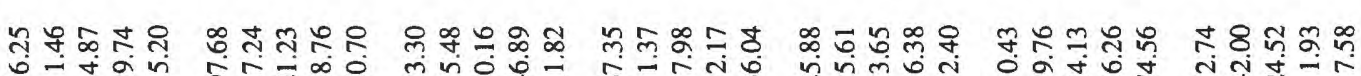

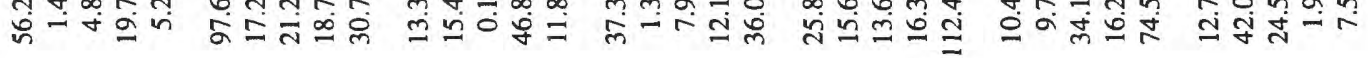

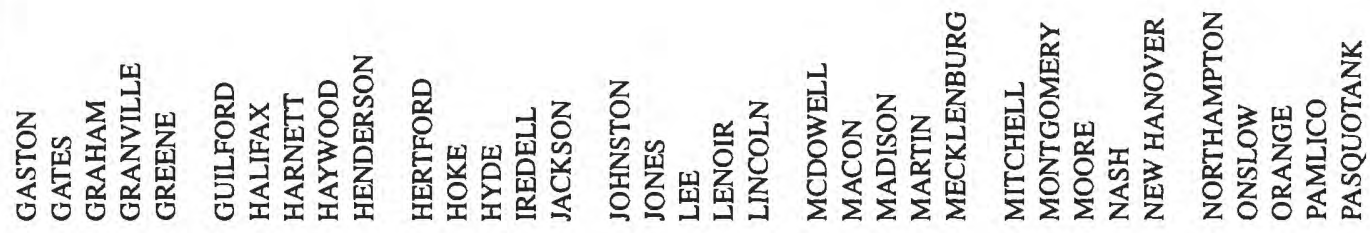




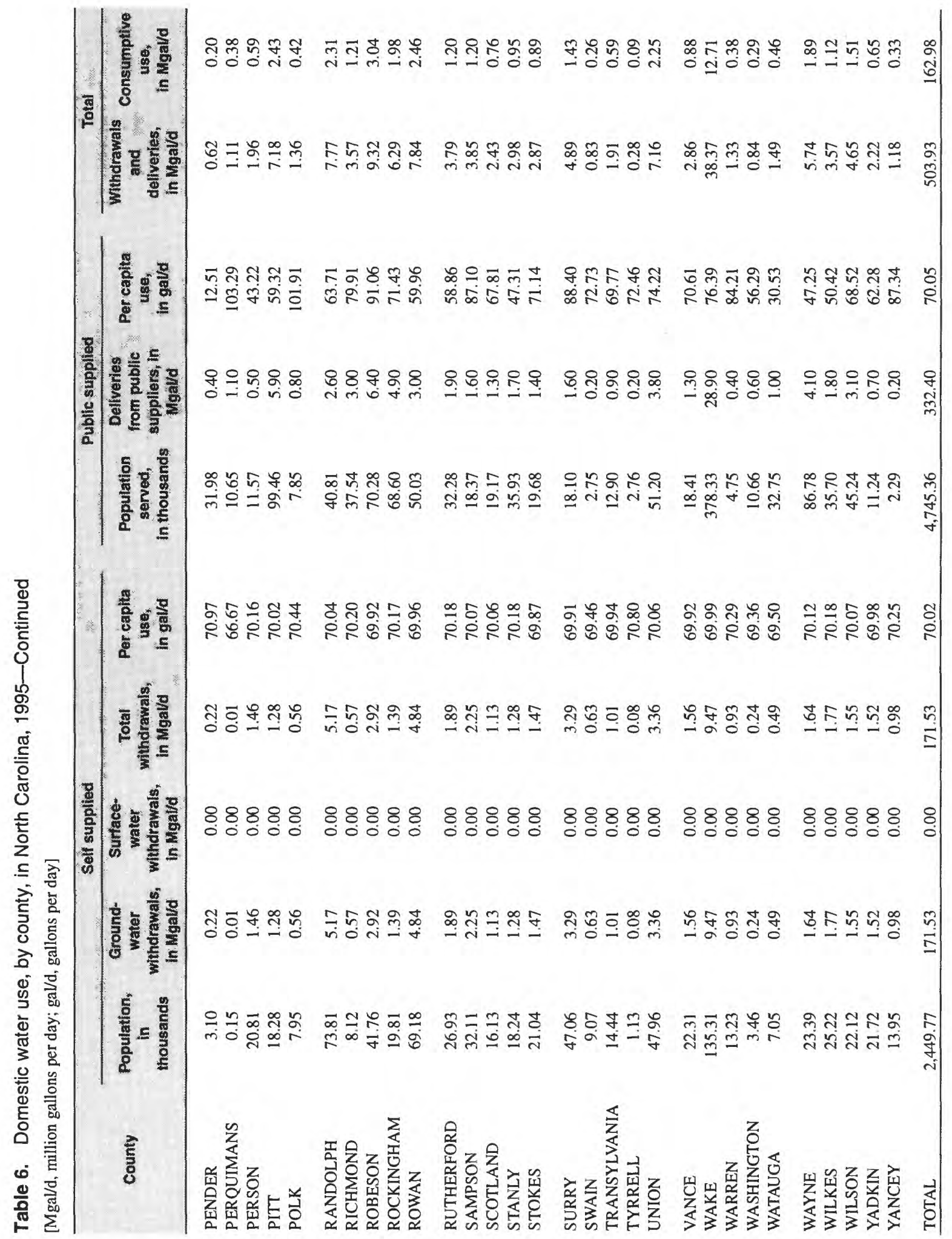



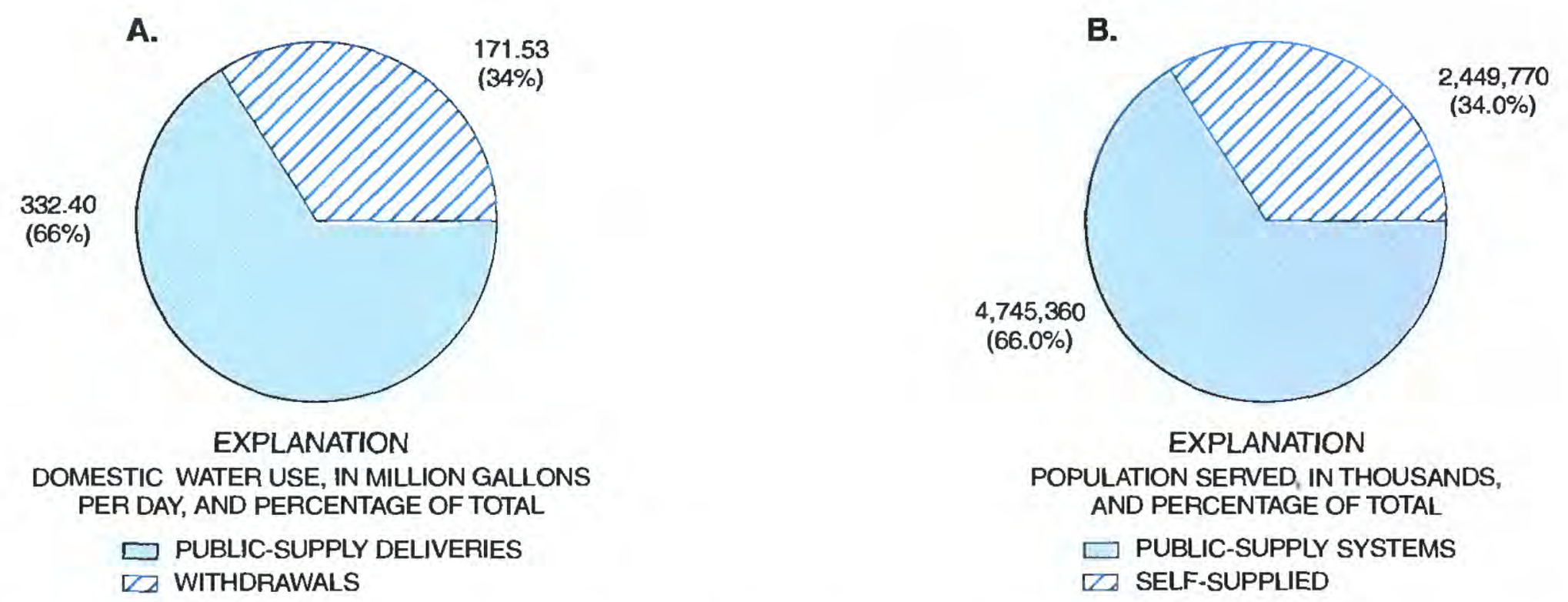

Figure 9. Domestic water use in North Carolina, 1995. (A) Public-supply deliveries and withdrawals, and (B) Population served by public suppliers and self-supplied sources.

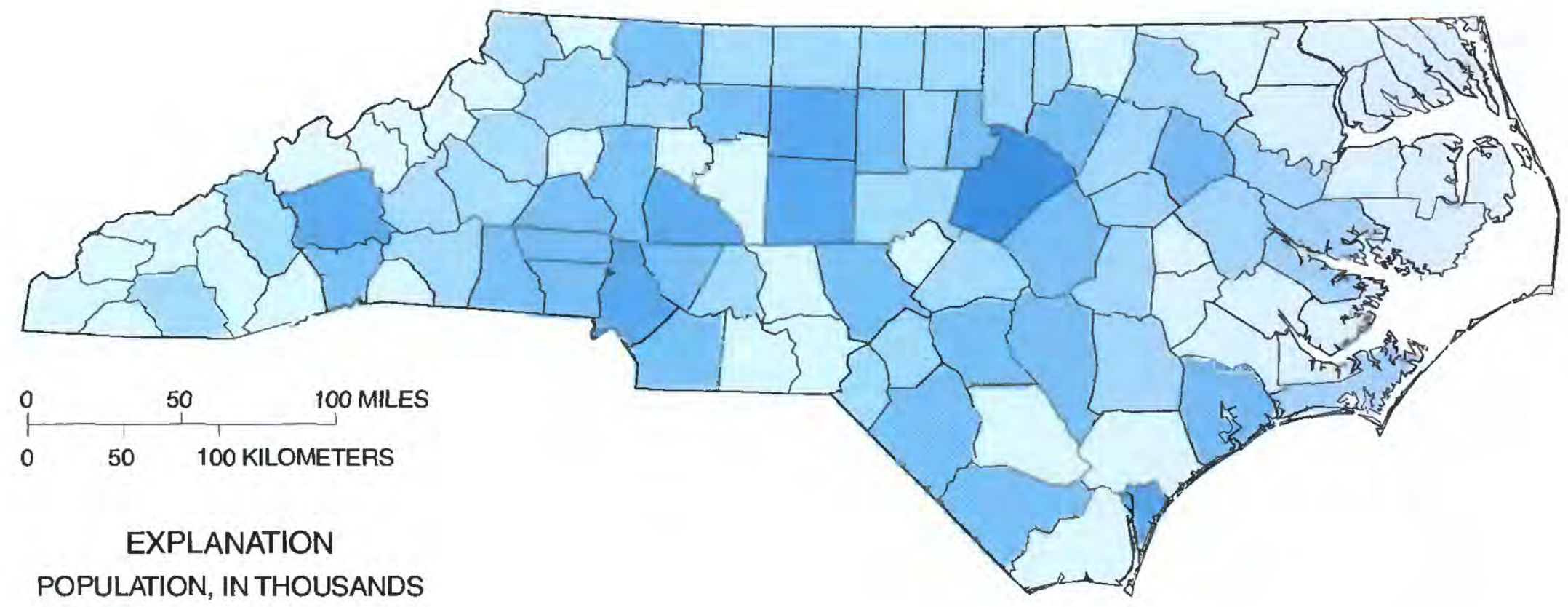

14.99 OR LESS

$15.00-29.99$

$30.00-59.99$

$6.00-119.99$

120 OR MORE

Figure 10. Population reliant on self-supplied water for domestic use, by county, in North Carolina, 1995. (Data from the U.S. Geological Survey National Data Storage and Retrieval System.) 
A. Total use (public-supply deliveries and withdrawals)

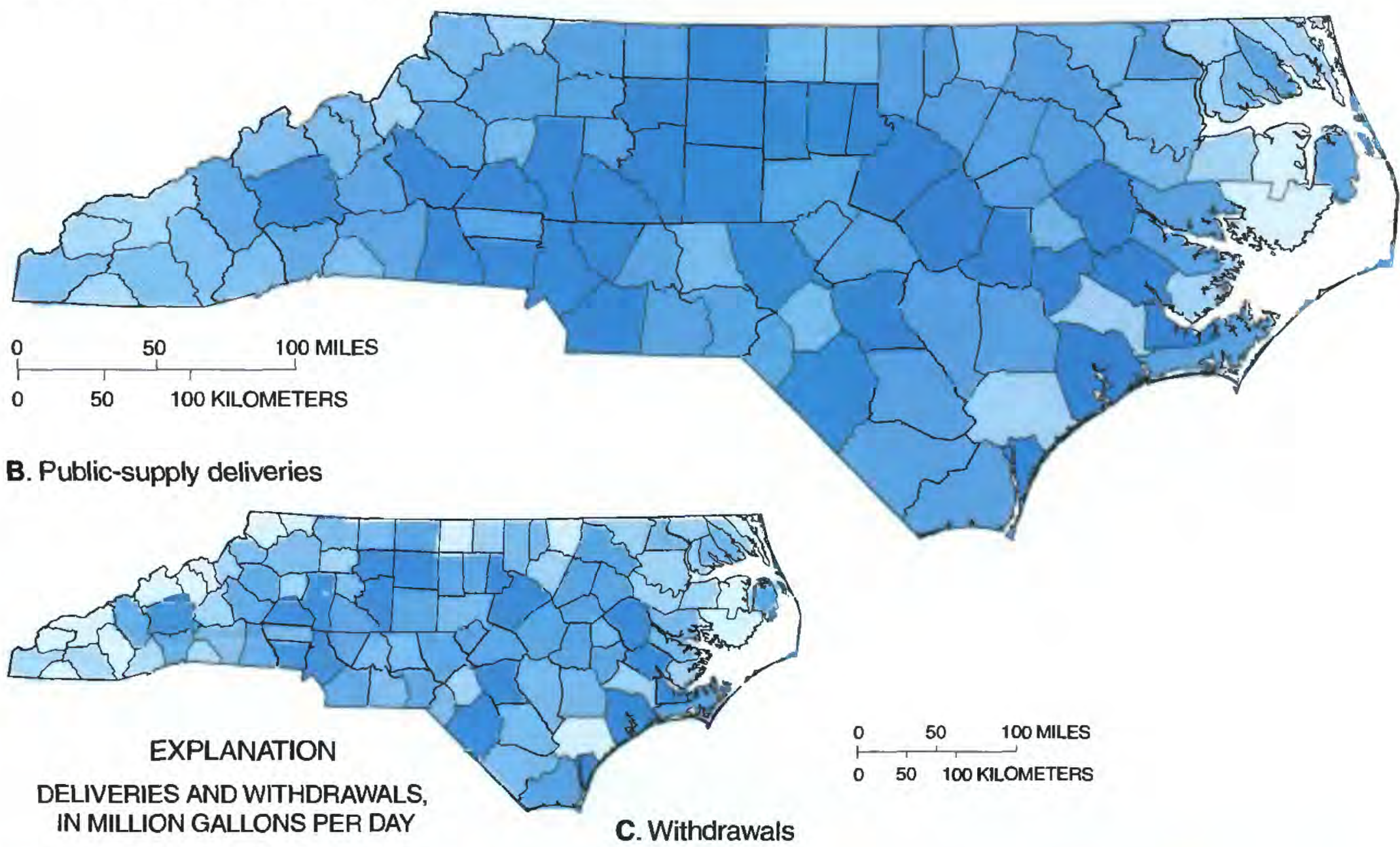
IN MILLION GALLONS PER DAY

0.49 OR LESS

$0.50-0.99$

$1.00-1.99$

$2.00-4.99$

5 OR MORE

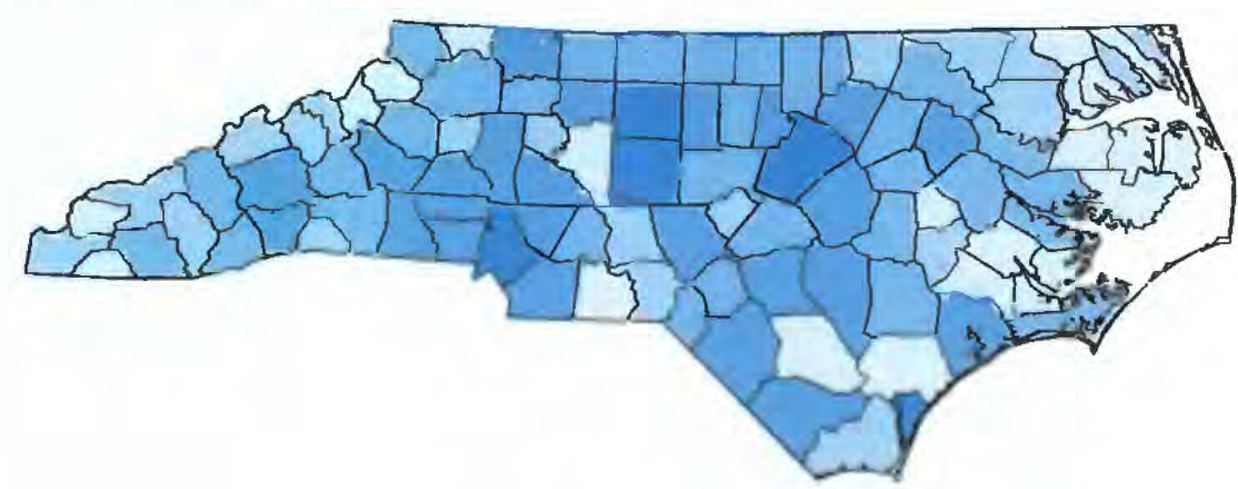

Figure 11. Domestic water use, by county, in North Carolina, 1995. (Data from the U.S. Geological Survey National Data Storage and Retrieval System.)

\section{Commercial Water Use}

In 1995, commercial water use in North Carolina was estimated at $146 \mathrm{Mgal} / \mathrm{d}$, which is less than 2 percent of the State's total water withdrawals (table 7). Withdrawals were estimated at $7.60 \mathrm{Mgal} / \mathrm{d}$. Selfsupplied commercial water use is difficult to estimate because withdrawals tend to be relatively small and water-use coefficients for commercial use vary between water-use coefficient sources. County withdrawals ranged from 0 to $0.38 \mathrm{Mgal} / \mathrm{d}$ (table 7; fig. 12). The source of self-supplied commercial water was assumed to be ground water.

Public-supply deliveries comprised 95 percent, or $138 \mathrm{Mgal} / \mathrm{d}$, of all commercial water use (fig. 13). Three counties received more than $10 \mathrm{Mgal} / \mathrm{d}$ of water from public suppliers for commercial useCumberland, Mecklenburg, and Wake Counties. 
Table 7. Commercial water use, in million gallons per day, by county, in North Carolina, 1995

\begin{tabular}{|c|c|c|c|c|c|c|}
\hline \multirow{2}{*}{ County } & \multicolumn{3}{|c|}{ Self-supplied withdrawals } & \multirow{2}{*}{$\begin{array}{l}\text { Deliveries } \\
\text { trom public } \\
\text { suppilers }\end{array}$} & \multicolumn{2}{|c|}{ Total use } \\
\hline & $\begin{array}{l}\text { Ground } \\
\text { water }\end{array}$ & $\begin{array}{l}\text { Surface } \\
\text { water }\end{array}$ & Total & & $\begin{array}{c}\text { Withdrawals } \\
\text { and } \\
\text { dellveries }\end{array}$ & $\begin{array}{c}\text { Consumptive } \\
\text { use }\end{array}$ \\
\hline ALAMANCE & 0.17 & 0.00 & 0.17 & 2.57 & 2.74 & 0.14 \\
\hline ALEXANDER & 0.01 & 0.00 & 0.01 & 0.07 & 0.08 & 0.00 \\
\hline ALLEGHANY & 0.05 & 0.00 & 0.05 & 0.04 & 0.09 & 0.00 \\
\hline ANSON & 0.00 & 0.00 & 0.00 & 0.13 & 0.13 & 0.01 \\
\hline ASHE & 0.07 & 0.00 & 0.07 & 0.11 & 0.18 & 0.01 \\
\hline AVERY & 0.11 & 0.00 & 0.11 & 0.10 & 0.21 & 0.01 \\
\hline BEAUFORT & 0.09 & 0.00 & 0.09 & 0.35 & 0.44 & 0.02 \\
\hline BERTIE & 0.02 & 0.00 & 0.02 & 0.09 & 0.11 & 0.01 \\
\hline BLADEN & 0.25 & 0.00 & 0.25 & 0.13 & 0.38 & 0.02 \\
\hline BRUNSWICK & 0.11 & 0.00 & 0.11 & 0.11 & 0.22 & 0.01 \\
\hline BUNCOMBE & 0.07 & 0.00 & 0.07 & 2.02 & 2.09 & 0.10 \\
\hline BURKE & 0.14 & 0.00 & 0.14 & 1.31 & 1.45 & 0.07 \\
\hline CABARRUS & 0.06 & 0.00 & 0.06 & 1.33 & 1.39 & 0.07 \\
\hline CALDWELL & 0.07 & 0.00 & 0.07 & 0.51 & 0.58 & 0.03 \\
\hline CAMDEN & 0.01 & 0.00 & 0.01 & 0.00 & 0.01 & 0.00 \\
\hline CARTERET & 0.12 & 0.00 & 0.12 & 1.21 & 1.33 & 0.07 \\
\hline CASWELL & 0.02 & 0.00 & 0.02 & 0.09 & 0.11 & 0.01 \\
\hline CATAWBA & 0.15 & 0.00 & 0.15 & 2.42 & 2.57 & 0.13 \\
\hline CHATHAM & 0.03 & 0.04 & 0.07 & 0.18 & 0.25 & 0.01 \\
\hline CHEROKEE & 0.04 & 0.00 & 0.04 & 0.24 & 0.28 & 0.01 \\
\hline CHOWAN & 0.00 & 0.00 & 0.00 & 0.44 & 0.44 & 0.02 \\
\hline CLAY & 0.03 & 0.00 & 0.03 & 0.05 & 0.08 & 0.00 \\
\hline CLEVELAND & 0.05 & 0.00 & 0.05 & 0.70 & 0.75 & 0.04 \\
\hline COLUMBUS & 0.11 & 0.00 & 0.11 & 0.49 & 0.60 & 0.03 \\
\hline CRAVEN & 0.08 & 0.00 & 0.08 & 1.48 & 1.56 & 0.08 \\
\hline CUMBERLAND & 0.18 & 0.00 & 0.18 & 10.53 & 10.71 & 0.54 \\
\hline CURRITUCK & 0.02 & 0.00 & 0.02 & 0.15 & 0.17 & 0.01 \\
\hline DARE & 0.05 & 0.00 & 0.05 & 0.50 & 0.55 & 0.03 \\
\hline DAVIDSON & 0.00 & 0.00 & 0.00 & 3.42 & 3.42 & 0.17 \\
\hline DAVIE & 0.01 & 0.00 & 0.01 & 0.23 & 0.24 & 0.01 \\
\hline DUPLIN & 0.04 & 0.00 & 0.04 & 0.41 & 0.45 & 0.02 \\
\hline DURHAM & 0.05 & 0.00 & 0.05 & 7.10 & 7.15 & 0.36 \\
\hline EDGECOMBE & 0.04 & 0.00 & 0.04 & 0.08 & 0.12 & 0.01 \\
\hline FORSYTH & 0.02 & 0.00 & 0.02 & 0.30 & 0.32 & 0.02 \\
\hline FRANKLIN & 0.06 & 0.00 & 0.06 & 0.30 & 0.36 & 0.02 \\
\hline GASTON & 0.00 & 0.00 & 0.00 & 4.07 & 4.07 & 0.20 \\
\hline GATES & 0.00 & 0.00 & 0.00 & 0.00 & 0.00 & 0.00 \\
\hline GRAHAM & 0.01 & 0.00 & 0.01 & 0.02 & 0.03 & 0.00 \\
\hline GRANVILLE & 0.02 & 0.00 & 0.02 & 0.79 & 0.81 & 0.04 \\
\hline GREENE & 0.00 & 0.00 & 0.00 & 0.17 & 0.17 & 0.01 \\
\hline GUILFORD & 0.38 & 0.00 & 0.38 & 7.47 & 7.85 & 0.39 \\
\hline HALIFAX & 0.05 & 0.00 & 0.05 & 0.80 & 0.85 & 0.04 \\
\hline HARNETT & 0.02 & 0.00 & 0.02 & 1.01 & 1.03 & 0.05 \\
\hline HAYWOOD & 0.08 & 0.00 & 0.08 & 1.00 & 1.08 & 0.05 \\
\hline HENDERSON & 0.21 & 0.00 & 0.21 & 1.14 & 1.35 & 0.07 \\
\hline HERTFORD & 0.04 & 0.00 & 0.04 & 0.28 & 0.32 & 0.02 \\
\hline HOKE & 0.01 & 0.00 & 0.01 & 0.06 & 0.07 & 0.00 \\
\hline HYDE & 0.02 & 0.00 & 0.02 & 0.06 & 0.08 & 0.00 \\
\hline IREDELL & 0.05 & 0.00 & 0.05 & 2.89 & 2.94 & 0.15 \\
\hline JACKSON & 0.11 & 0.00 & 0.11 & 0.65 & 0.76 & 0.04 \\
\hline
\end{tabular}


Table 7. Commercial water use, in million gallons per day, by county, in North Carolina, 1995-Continued

\begin{tabular}{|c|c|c|c|c|c|c|}
\hline & Se & aplied with & & & Tota & use \\
\hline County & $\begin{array}{l}\text { Ground } \\
\text { water }\end{array}$ & $\begin{array}{l}\text { Surface } \\
\text { water }\end{array}$ & Total & $\begin{array}{l}\text { from public } \\
\text { suppliers }\end{array}$ & $\begin{array}{c}\text { Withdrawals } \\
\text { and } \\
\text { deliveries }\end{array}$ & $\begin{array}{c}\text { Consumptive } \\
\text { use }\end{array}$ \\
\hline JOHNSTON & 0.11 & 0.00 & 0.11 & 0.65 & 0.76 & 0.04 \\
\hline JONES & 0.00 & 0.00 & 0.00 & 0.05 & 0.05 & 0.00 \\
\hline LEE & 0.00 & 0.00 & 0.00 & 0.03 & 0.03 & 0.00 \\
\hline LENOIR & 0.03 & 0.00 & 0.03 & 0.47 & 0.50 & 0.03 \\
\hline LINCOLN & 0.05 & 0.00 & 0.05 & 0.63 & 0.68 & 0.03 \\
\hline MCDOWELL & 0.21 & 0.00 & 0.21 & 0.19 & 0.40 & 0.04 \\
\hline MACON & 0.08 & 0.00 & 0.08 & 0.51 & 0.59 & 0.01 \\
\hline MADISON & 0.02 & 0.00 & 0.02 & 0.04 & 0.06 & 0.00 \\
\hline MARTIN & 0.06 & 0.00 & 0.06 & 0.38 & 0.44 & 0.01 \\
\hline MECKLENBURG & 0.34 & 0.00 & 0.34 & 25.52 & 25.86 & 1.29 \\
\hline MITCHELL & 0.04 & 0.00 & 0.04 & 0.34 & 0.38 & 0.02 \\
\hline MONTGOMERY & 0.02 & 0.00 & 0.02 & 0.43 & 0.45 & 0.02 \\
\hline MOORE & 0.13 & 0.00 & 0.13 & 1.17 & 1.30 & 0.06 \\
\hline NASH & 0.15 & 0.00 & 0.15 & 2.56 & 2.71 & 0.14 \\
\hline NEW HANOVER & 0.17 & 0.01 & 0.18 & 2.09 & 2.27 & 0.11 \\
\hline NORTHAMPTON & 0.02 & 0.00 & 0.02 & 0.12 & 0.14 & 0.01 \\
\hline ONSLOW & 0.03 & 0.00 & 0.03 & 0.33 & 0.36 & 0.02 \\
\hline ORANGE & 0.03 & 0.00 & 0.03 & 3.02 & 3.05 & 0.15 \\
\hline PAMLICO & 0.00 & 0.00 & 0.00 & 0.07 & 0.07 & 0.00 \\
\hline PASQUOTANK & 0.00 & 0.00 & 0.00 & 0.54 & 0.54 & 0.03 \\
\hline PENDER & 0.11 & 0.00 & 0.11 & 0.20 & 0.31 & 0.02 \\
\hline PERQUIMANS & 0.01 & 0.00 & 0.01 & 0.05 & 0.06 & 0.00 \\
\hline PERSON & 0.03 & 0.01 & 0.04 & 0.35 & 0.39 & 0.02 \\
\hline PITT & 0.03 & 0.00 & 0.03 & 4.03 & 4.06 & 0.20 \\
\hline POLK & 0.02 & 0.00 & 0.02 & 0.09 & 0.11 & 0.01 \\
\hline RANDOLPH & 0.12 & 0.00 & 0.12 & 0.32 & 0.44 & 0.02 \\
\hline RICHMOND & 0.01 & 0.00 & 0.01 & 1.29 & 1.30 & 0.07 \\
\hline ROBESON & 0.06 & 0.00 & 0.06 & 0.48 & 0.54 & 0.03 \\
\hline ROCKINGHAM & 0.06 & 0.00 & 0.06 & 5.96 & 6.02 & 0.16 \\
\hline ROWAN & 0.13 & 0.10 & 0.23 & 0.71 & 0.94 & 0.19 \\
\hline RUTHERFORD & 0.13 & 0.00 & 0.13 & 0.75 & 0.88 & 0.04 \\
\hline SAMPSON & 0.15 & 0.00 & 0.15 & 0.48 & 0.63 & 0.03 \\
\hline SCOTLAND & 0.01 & 0.00 & 0.01 & 0.63 & 0.64 & 0.03 \\
\hline STANLY & 0.06 & 0.00 & 0.06 & 1.96 & 2.02 & 0.10 \\
\hline STOKES & 0.27 & 0.00 & 0.27 & 0.07 & 0.34 & 0.02 \\
\hline SURRY & 0.18 & 0.00 & 0.18 & 0.88 & 1.06 & 0.05 \\
\hline SWAIN & 0.16 & 0.00 & 0.16 & 0.10 & 0.26 & 0.01 \\
\hline TRANSYLVANIA & 0.13 & 0.10 & 0.23 & 0.05 & 0.28 & 0.01 \\
\hline TYRRELL & 0.00 & 0.00 & 0.00 & 0.03 & 0.03 & 0.00 \\
\hline UNION & 0.03 & 0.00 & 0.03 & 3.60 & 3.63 & 0.18 \\
\hline VANCE & 0.04 & 0.00 & 0.04 & 0.51 & 0.55 & 0.03 \\
\hline WAKE & 0.22 & 0.03 & 0.25 & 11.03 & 11.28 & 0.56 \\
\hline WARREN & 0.02 & 0.00 & 0.02 & 0.04 & 0.06 & 0.00 \\
\hline WASHINGTON & 0.00 & 0.00 & 0.00 & 0.12 & 0.12 & 0.01 \\
\hline WATAUGA & 0.14 & 0.00 & 0.14 & 1.25 & 1.39 & 0.07 \\
\hline WAYNE & 0.04 & 0.00 & 0.04 & 1.64 & 1.68 & 0.08 \\
\hline WILKES & 0.07 & 0.00 & 0.07 & 1.42 & 1.49 & 0.07 \\
\hline WILSON & 0.06 & 0.00 & 0.06 & 0.94 & 1.00 & 0.05 \\
\hline YADKIN & 0.10 & 0.00 & 0.10 & 0.20 & 0.30 & 0.01 \\
\hline YANCEY & 0.10 & 0.00 & 0.10 & 0.08 & 0.18 & 0.01 \\
\hline TOTAL & 7.31 & 0.29 & 7.60 & 138.00 & 145.60 & 7.24 \\
\hline
\end{tabular}




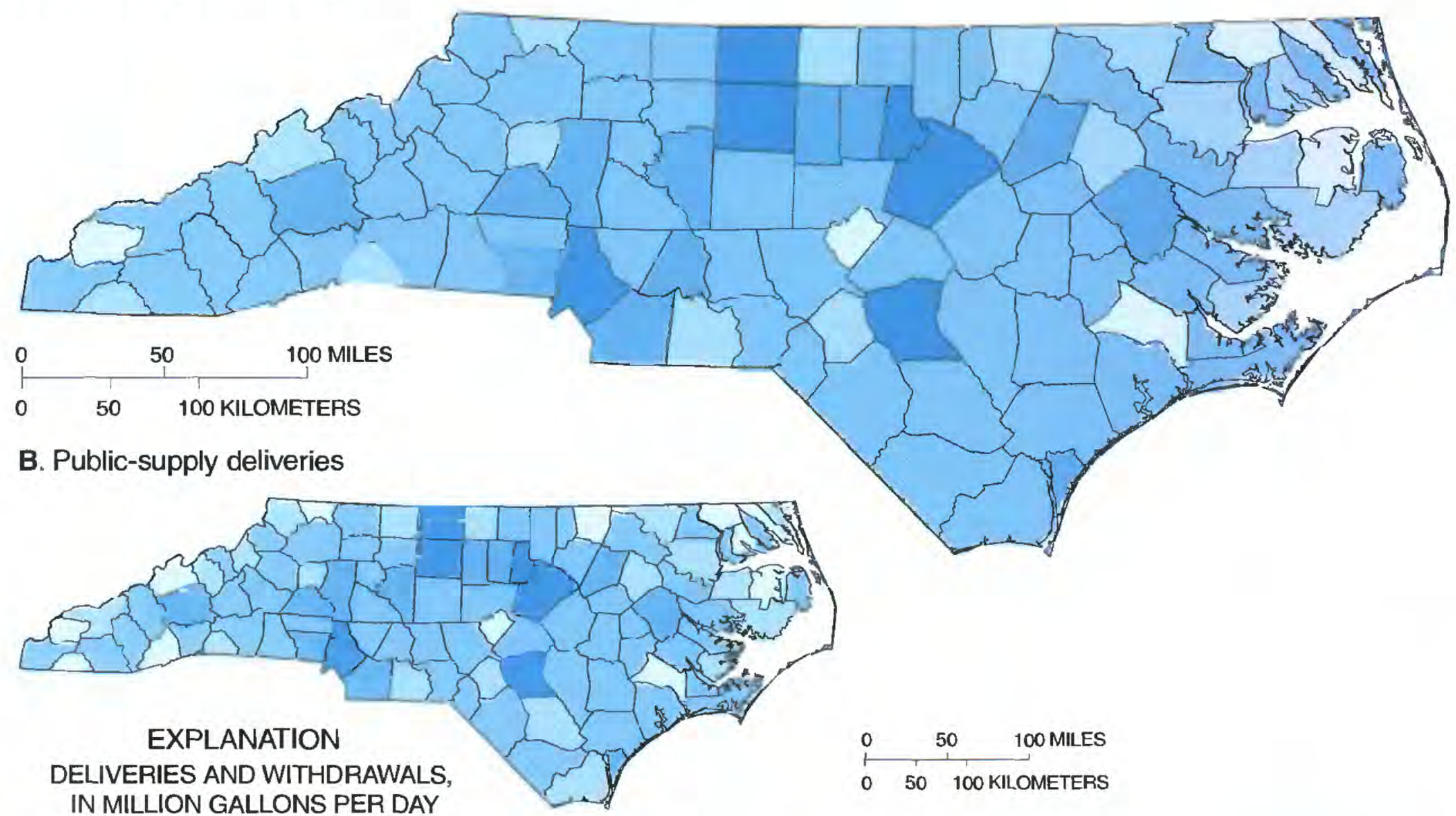

0.0599 OR LESS

C. Withdrawals

$0.06-0.1499$

$0.15-1.99$

$2.00-4.99$

5 OR MORE

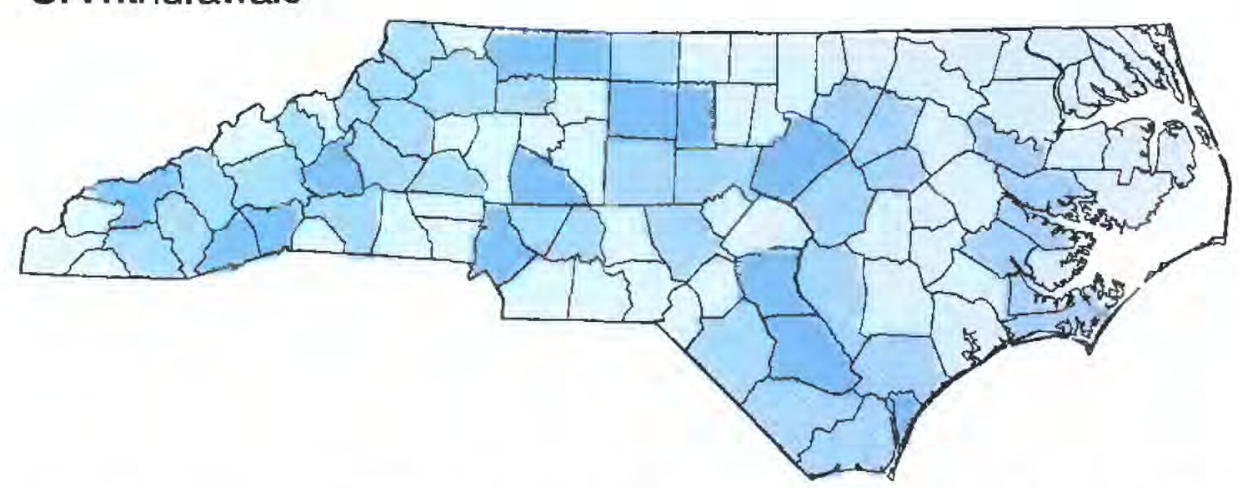

Figure 12. Commercial water use, by county, in North Carolina, 1995. (Data from the U.S. Geological Survey National Data Storage and Retrieval System.)

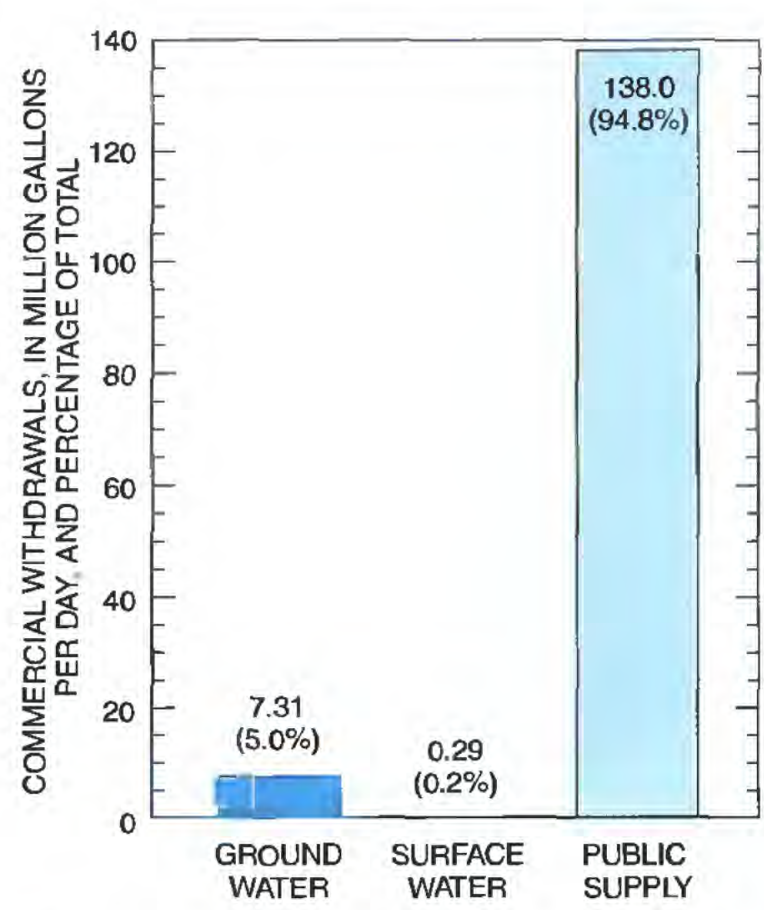

Figure 13. Source of water for commercial use in North Carolina, 1995.

\section{Industrial Water Use}

Industrial water use (including public-supply deliveries) in North Carolina, amounted to 6 percent, or $562 \mathrm{Mgal} / \mathrm{d}$, of all withdrawals in the State (table 8). Withdrawals for industrial water use were approximately $369 \mathrm{Mgal} / \mathrm{d}$. Of the industrial water use, 55 percent was from surface water. Public suppliers delivered about 34 percent of the total water used for industry (fig. 14). Harnett, Gaston, and Guilford Counties accounted for 33 percent of all water used by industries. Withdrawals for these counties accounted for almost 45 percent of the total for this category. Textiles, chemicals, and plastic products were the main industries in these counties. Total water use (deliveries and withdrawals), by county, varied geographically and ranged from 0 to $84 \mathrm{Mgal} / \mathrm{d}$ (table 8 ; fig. 15). 
Table 8. Industrial water use, in million gallons per day, by county, in North Carolina, 1995

\begin{tabular}{|c|c|c|c|c|c|c|}
\hline \multirow[b]{2}{*}{ County } & \multicolumn{3}{|c|}{ Self-supplled withdrawals } & \multirow[b]{2}{*}{$\begin{array}{l}\text { Dellveries } \\
\text { trom public } \\
\text { suppiliters }\end{array}$} & \multicolumn{2}{|c|}{ Total use } \\
\hline & $\begin{array}{l}\text { Ground } \\
\text { water }\end{array}$ & $\begin{array}{l}\text { Surtace } \\
\text { water }\end{array}$ & Total & & $\begin{array}{c}\text { Withdrawals } \\
\text { and } \\
\text { deltuerles }\end{array}$ & $\begin{array}{l}\text { Consump- } \\
\text { thve use }\end{array}$ \\
\hline ALAMANCE & 0.57 & 4.68 & 5.25 & 7.33 & 12.58 & 2.52 \\
\hline ALEXANDER & 0.00 & 0.00 & 0.00 & 0.14 & 0.14 & 0.03 \\
\hline ALLEGHANY & 0.01 & 0.08 & 0.09 & 0.00 & 0.09 & 0.02 \\
\hline ANSON & 2.55 & 2.02 & 4.57 & 1.68 & 6.25 & 1.25 \\
\hline ASHE & 0.00 & 0.00 & 0.00 & 0.05 & 0.05 & 0.01 \\
\hline AVERY & 0.35 & 0.18 & 0.53 & 0.00 & 0.53 & 0.11 \\
\hline BEAUFORT & 0.01 & 0.18 & 0.19 & 0.23 & 0.42 & 0.08 \\
\hline BERTIE & 0.02 & 0.31 & 0.33 & 0.02 & 0.35 & 0.07 \\
\hline BLADEN & 1.23 & 0.93 & 2.16 & 0.25 & 2.41 & 0.48 \\
\hline BRUNSWICK & 0.23 & 0.34 & 0.57 & 6.02 & 6.59 & 1.32 \\
\hline BUNCOMBE & 0.56 & 2.26 & 2.82 & 7.03 & 9.85 & 1.97 \\
\hline BURKE & 2.22 & 5.09 & 7.31 & 11.22 & 18.53 & 3.71 \\
\hline CABARRUS & 2.75 & 14.01 & 16.76 & 1.75 & 18.51 & 3.70 \\
\hline CALDWELL & 0.35 & 1.26 & 1.61 & 1.51 & 3.12 & 0.62 \\
\hline CAMDEN & 0.00 & 0.00 & 0.00 & 0.00 & 0.00 & 0.00 \\
\hline CARTERET & 0.00 & 0.01 & 0.01 & 0.04 & 0.05 & 0.01 \\
\hline CASWELL & 0.00 & 0.00 & 0.00 & 0.03 & 0.03 & 0.01 \\
\hline CATAWBA & 2.89 & 2.69 & 5.58 & 7.74 & 13.32 & 2.66 \\
\hline CHATHAM & 0.06 & 0.36 & 0.42 & 1.52 & 1.94 & 0.39 \\
\hline CHEROKEE & 0.00 & 0.00 & 0.00 & 0.29 & 0.29 & 0.06 \\
\hline CHOWAN & 0.13 & 0.04 & 0.17 & 0.20 & 0.37 & 0.07 \\
\hline CLAY & 0.00 & 0.00 & 0.00 & 0.01 & 0.01 & 0.00 \\
\hline CLEVELAND & 0.61 & 1.15 & 1.76 & 4.99 & 6.75 & 1.35 \\
\hline COLUMBUS & 0.05 & 0.93 & 0.98 & 0.14 & 1.12 & 0.22 \\
\hline CRAVEN & 0.00 & 0.04 & 0.04 & 3.10 & 3.14 & 0.63 \\
\hline CUMBERLAND & 0.38 & 1.17 & 1.55 & 2.77 & 4.32 & 0.86 \\
\hline CURRITUCK & 0.00 & 0.00 & 0.00 & 0.00 & 0.00 & 0.00 \\
\hline DARE & 0.00 & 0.00 & 0.00 & 0.01 & 0.01 & 0.00 \\
\hline DAVIDSON & 0.66 & 3.87 & 4.53 & 1.02 & 5.55 & 1.11 \\
\hline DAVIE & 0.05 & 0.07 & 0.12 & 0.34 & 0.46 & 0.09 \\
\hline DUPLIN & 0.20 & 0.82 & 1.02 & 2.84 & 3.86 & 0.77 \\
\hline DURHAM & 0.25 & 3.75 & 4.00 & 1.30 & 5.30 & 1.06 \\
\hline EDGECOMBE & 0.14 & 1.21 & 1.35 & 1.01 & 2.36 & 0.47 \\
\hline FORSYTH & 0.60 & 7.95 & 8.55 & 24.58 & 33.13 & 6.63 \\
\hline FRANKLIN & 0.02 & 0.01 & 0.03 & 0.32 & 0.35 & 0.07 \\
\hline GASTON & 1.07 & 44.13 & 45.20 & 12.31 & 57.51 & 11.50 \\
\hline GATES & 0.00 & 0.00 & 0.00 & 0.00 & 0.00 & 0.00 \\
\hline GRAHAM & 0.00 & 0.00 & 0.00 & 0.06 & 0.06 & 0.01 \\
\hline GRANVILLE & 0.68 & 0.54 & 1.22 & 0.59 & 1.81 & 0.36 \\
\hline GREENE & 0.00 & 0.00 & 0.00 & 0.09 & 0.09 & 0.02 \\
\hline GUILFORD & 4.36 & 30.08 & 34.44 & 10.01 & 44.45 & 8.89 \\
\hline HALIFAX & 0.19 & 1.57 & 1.76 & 2.23 & 3.99 & 0.80 \\
\hline HARNETT & 2.93 & 81.21 & 84.14 & 0.19 & 84.33 & 16.87 \\
\hline HAYWOOD & 0.13 & 26.04 & 26.17 & 0.00 & 26.17 & 5.23 \\
\hline HENDERSON & 0.31 & 1.69 & 2.00 & 0.96 & 2.96 & 0.59 \\
\hline HERTFORD & 0.00 & 0.00 & 0.00 & 0.10 & 0.10 & 0.02 \\
\hline HOKE & 0.00 & 0.14 & 0.14 & 1.32 & 1.46 & 0.29 \\
\hline HYDE & 0.01 & 0.02 & 0.03 & 0.00 & 0.03 & 0.01 \\
\hline IREDELL & 0.65 & 6.81 & 7.46 & 0.64 & 8.10 & 1.62 \\
\hline JACKSON & 0.03 & 0.08 & 0.11 & 0.11 & 0.22 & 0.04 \\
\hline
\end{tabular}


Table 8. Industrial water use, in million gallons per day, by county, in North Carolina, 1995-Continued

\begin{tabular}{|c|c|c|c|c|c|c|}
\hline \multirow[b]{2}{*}{ County } & \multicolumn{3}{|c|}{ Sell-supplied withdrawals } & \multirow{2}{*}{$\begin{array}{l}\text { Dellverles } \\
\text { trom public } \\
\text { stuppllers }\end{array}$} & \multicolumn{2}{|c|}{ Total wase } \\
\hline & $\begin{array}{l}\text { Ground } \\
\text { water }\end{array}$ & $\begin{array}{l}\text { Surface } \\
\text { water }\end{array}$ & Total & & $\begin{array}{c}\text { Withdrawale } \\
\text { and } \\
\text { dellveries }\end{array}$ & $\begin{array}{l}\text { Consump } \\
\text { tive use }\end{array}$ \\
\hline JOHNSTON & 0.04 & 0.02 & 0.06 & 0.55 & 0.61 & 0.12 \\
\hline JONES & 0.67 & 2.15 & 2.82 & 0.00 & 2.82 & 0.56 \\
\hline LEE & 0.00 & 0.00 & 0.00 & 1.53 & 1.53 & 0.31 \\
\hline LENOIR & 0.07 & 0.18 & 0.25 & 1.23 & 1.48 & 0.30 \\
\hline LINCOLN & 0.01 & 0.51 & 0.52 & 1.67 & 2.19 & 0.44 \\
\hline MCDOWELL & 1.46 & 0.19 & 1.65 & 0.69 & 2.34 & 0.35 \\
\hline MACON & 0.03 & 0.16 & 0.19 & 0.08 & 0.27 & 0.04 \\
\hline MADISON & 0.01 & 0.01 & 0.02 & 0.00 & 0.02 & 0.00 \\
\hline MARTIN & 0.00 & 0.00 & 0.00 & 1.16 & 1.16 & 0.14 \\
\hline MECKLENBURG & 0.86 & 4.57 & 5.43 & 0.63 & 6.06 & 1.21 \\
\hline MITCHELL & 0.00 & 0.00 & 0.00 & 0.03 & 0.03 & 0.01 \\
\hline MONTGOMERY & 0.01 & 0.03 & 0.04 & 0.62 & 0.66 & 0.13 \\
\hline MOORE & 0.01 & 0.00 & 0.01 & 0.96 & 0.97 & 0.19 \\
\hline $\mathrm{NASH}$ & 0.19 & 1.57 & 1.76 & 5.31 & 7.07 & 1.41 \\
\hline NEW HANOVER & 0.13 & 0.30 & 0.43 & 0.44 & 0.87 & 0.17 \\
\hline NORTHAMPTON & 0.00 & 0.02 & 0.02 & 0.34 & 0.36 & 0.07 \\
\hline ONSLOW & 0.00 & 0.00 & 0.00 & 0.05 & 0.05 & 0.01 \\
\hline ORANGE & 0.00 & 0.00 & 0.00 & 0.75 & 0.75 & 0.15 \\
\hline PAMLICO & 0.00 & 0.00 & 0.00 & 0.00 & 0.00 & 0.00 \\
\hline PASQUOTANK & 0.03 & 0.03 & 0.06 & 0.16 & 0.22 & 0.04 \\
\hline PENDER & 0.00 & 0.00 & 0.00 & 0.00 & 0.00 & 0.00 \\
\hline PERQUIMANS & 0.00 & 0.00 & 0.00 & 0.00 & 0.00 & 0.00 \\
\hline PERSON & 0.21 & 0.50 & 0.71 & 2.84 & 3.55 & 0.71 \\
\hline PITT & 3.76 & 2.09 & 5.85 & 2.35 & 8.20 & 1.64 \\
\hline POLK & 0.00 & 0.01 & 0.01 & 0.23 & 0.24 & 0.05 \\
\hline RANDOLPH & 1.70 & 1.51 & 3.21 & 3.60 & 6.81 & 1.36 \\
\hline RICHMOND & 1.99 & 0.21 & 2.20 & 1.38 & 3.58 & 0.72 \\
\hline ROBESON & 1.48 & 4.11 & 5.59 & 8.80 & 14.39 & 2.88 \\
\hline ROCKINGHAM & 0.02 & 0.77 & 0.79 & 9.46 & 10.25 & 1.60 \\
\hline ROWAN & 0.07 & 1.95 & 2.02 & 5.85 & 7.87 & 2.03 \\
\hline RUTHERFORD & 0.26 & 10.71 & 10.97 & 6.11 & 17.08 & 3.42 \\
\hline SAMPSON & 0.00 & 0.05 & 0.05 & 0.25 & 0.30 & 0.06 \\
\hline SCOTLAND & 2.92 & 0.03 & 2.95 & 0.55 & 3.50 & 0.70 \\
\hline STANLY & 0.08 & 0.53 & 0.61 & 2.50 & 3.11 & 0.62 \\
\hline STOKES & 0.00 & 0.00 & 0.00 & 0.00 & 0.00 & 0.00 \\
\hline SURRY & 2.96 & 7.30 & 10.26 & 4.10 & 14.36 & 2.87 \\
\hline SWAIN & 0.00 & 0.00 & 0.00 & 0.02 & 0.02 & 0.00 \\
\hline TRANSYLVANIA & 0.00 & 0.02 & 0.02 & 0.00 & 0.02 & 0.00 \\
\hline TYRRELL & 0.00 & 0.00 & 0.00 & 0.00 & 0.00 & 0.00 \\
\hline UNION & 0.19 & 4.83 & 5.02 & 1.24 & 6.26 & 1.23 \\
\hline VANCE & 11.48 & 2.25 & 13.73 & 0.42 & 14.15 & 2.83 \\
\hline WAKE & 1.63 & 6.28 & 7.91 & 2.48 & 10.39 & 2.08 \\
\hline WARREN & 0.01 & 0.05 & 0.06 & 0.02 & 0.08 & 0.02 \\
\hline WASHINGTON & 0.00 & 0.00 & 0.00 & 0.01 & 0.01 & 0.00 \\
\hline WATAUGA & 0.00 & 0.00 & 0.00 & 0.06 & 0.06 & 0.01 \\
\hline WAYNE & 0.89 & 5.37 & 6.26 & 1.57 & 7.83 & 1.57 \\
\hline WILKES & 0.00 & 0.00 & 0.00 & 2.84 & 2.84 & 0.57 \\
\hline WILSON & 0.68 & 1.91 & 2.59 & 2.01 & 4.60 & 0.92 \\
\hline YADKIN & 0.00 & 0.00 & 0.00 & 0.30 & 0.30 & 0.06 \\
\hline YANCEY & 0.00 & 0.00 & 0.00 & 0.09 & 0.09 & 0.02 \\
\hline TOTAL & 61.10 & 307.94 & 369.04 & 193.37 & 562.41 & 112.22 \\
\hline
\end{tabular}


Industrial water use is difficult to estimate because coefficients vary by product, plant facility, and process. For 1995, water use for the industrial category was estimated as follows. Surveys were received from 200 randomly selected facilities of the more than 2,200 water-using industrial facilities listed in the North Carolina Manufacturing Firms, 1995 Directory. Data from the survey were incorporated into a statistical model relating water use to the number of employees, facility SIC (standard industrial classification) code, and county population density (Gregory E. Schwarz, U.S. Geological Survey, written commun., 1997). Results of the analysis indicate that total water use is greater in facilities with a large employment base, but is lower in facilities with a comparable employment base that produce metal or plastic products. A large

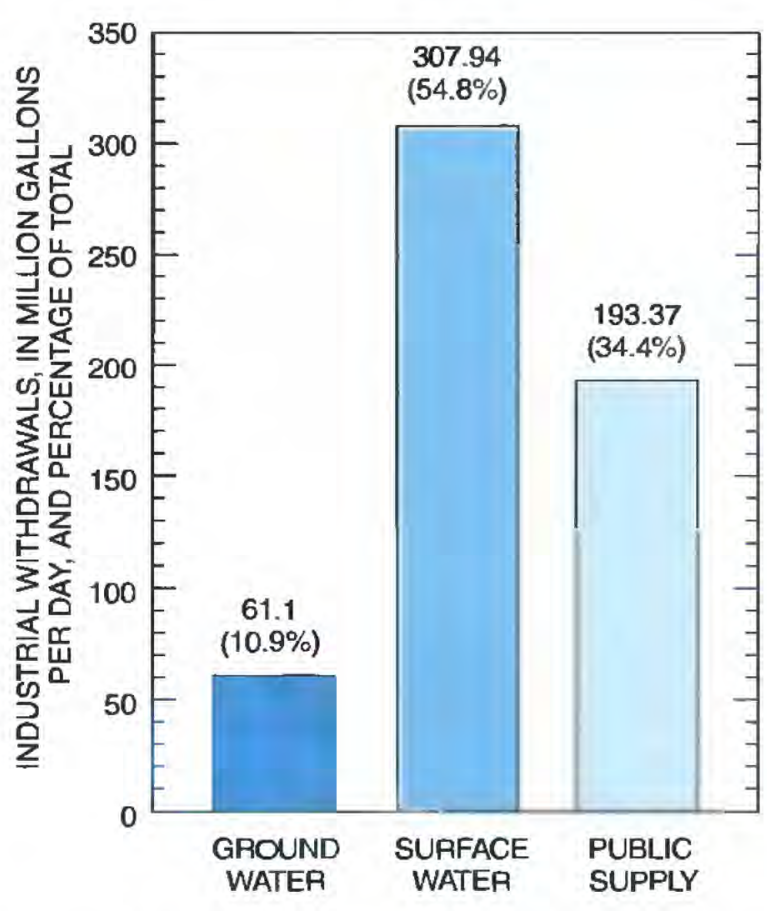

Figure 14. Source of water for industrial use in North Carolina, 1995.

A. Total use (public-supply deliveries and withdrawals)

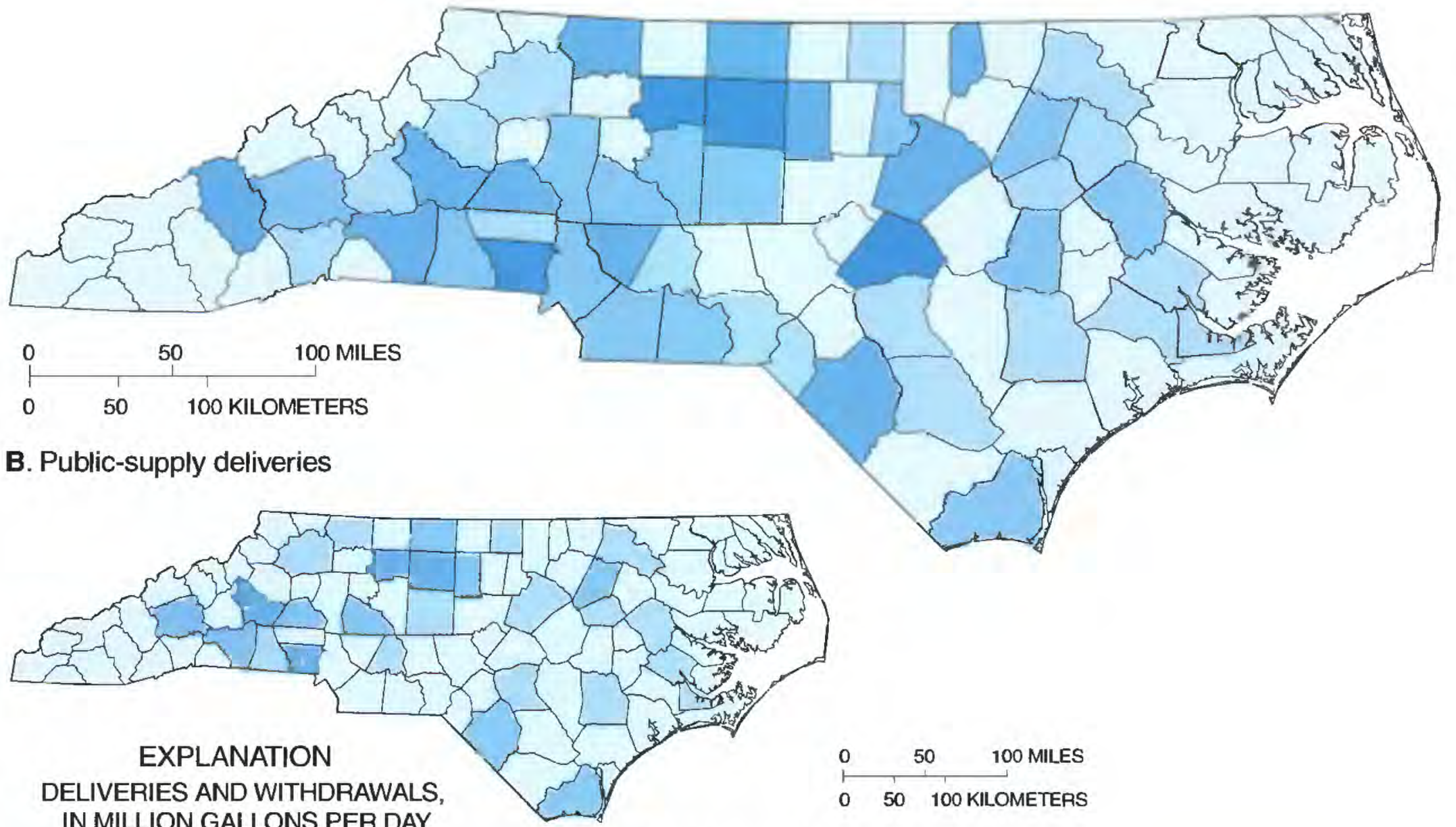

IN MILLION GALLONS PER DAY
1.99 OR LESS
$2.00-4.99$
$5.00-9.99$
$10.00-29.99$
30 OR MORE

\section{Withdrawals}

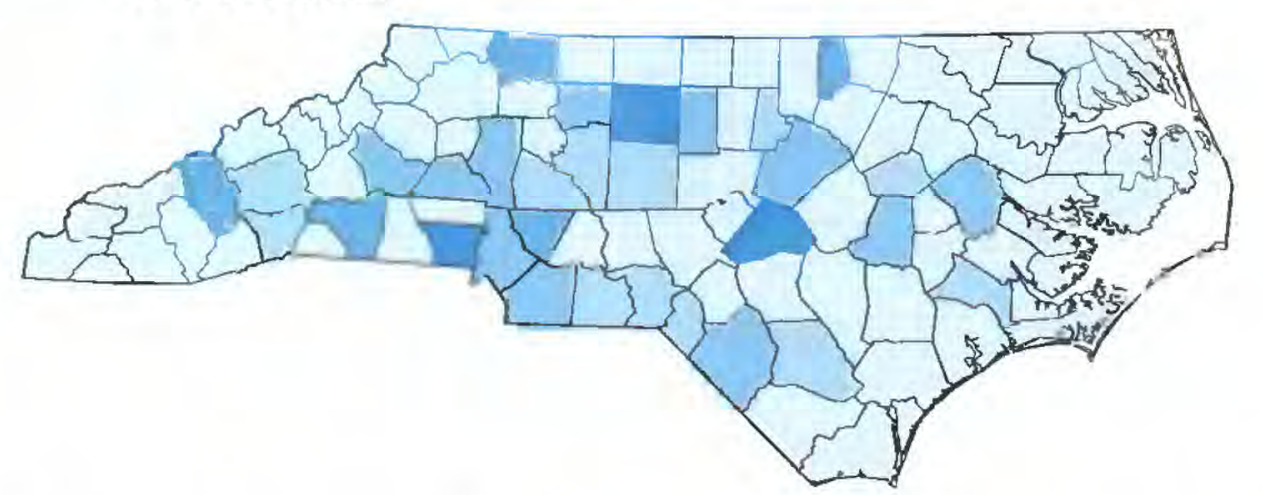

Figure 15. Industrial water use, by county, in North Carolina, 1995. (Data from the U.S. Geological Survey National Data Storage and Retrieval System.) 
proportion of water delivered to a facility comes from public supplies if the facility (1) has a large employment base but relatively small water needs, (2) produces fabric or food products, or (3) is in a county with high population density. For self-supplied facilities, withdrawals are more likely to come from surface water where large amounts of water are needed. Finally, the percentage of water consumed is positively related to the facility employment and if the facility produces cement, petrol, or wood products. Overall, using employment and other readily obtained facility characteristics as a predictor of water use is a viable method of computing industrial water-use estimates.

\section{Mining Water Use}

Water withdrawals for mining accounted for less than 0.2 percent of all water withdrawn in North Carolina in 1995. Mining operations are generally self supplied. Total withdrawals for mining were about $16 \mathrm{Mgal} / \mathrm{d}$, of which 73 percent, or $12 \mathrm{Mgal} / \mathrm{d}$, was from ground water (table 9; fig. 16). Mining operations in Beaufort County accounted for the largest withdrawals in the State with withdrawals of $9 \mathrm{Mgal} / \mathrm{d}$, or 57 percent of all withdrawals for this category (table 9; fig. 17). Some of the ground water withdrawn was from wells, but most was from ground-water discharge into excavation pits from which the water was later withdrawn for use. Estimates published in this report do not include dewatering. However, the dewatering discharges large amounts of water to streams and rivers. In Beaufort County, mining operations discharged nearly $22 \mathrm{Mgal} / \mathrm{d}$ in 1995 from dewatering.

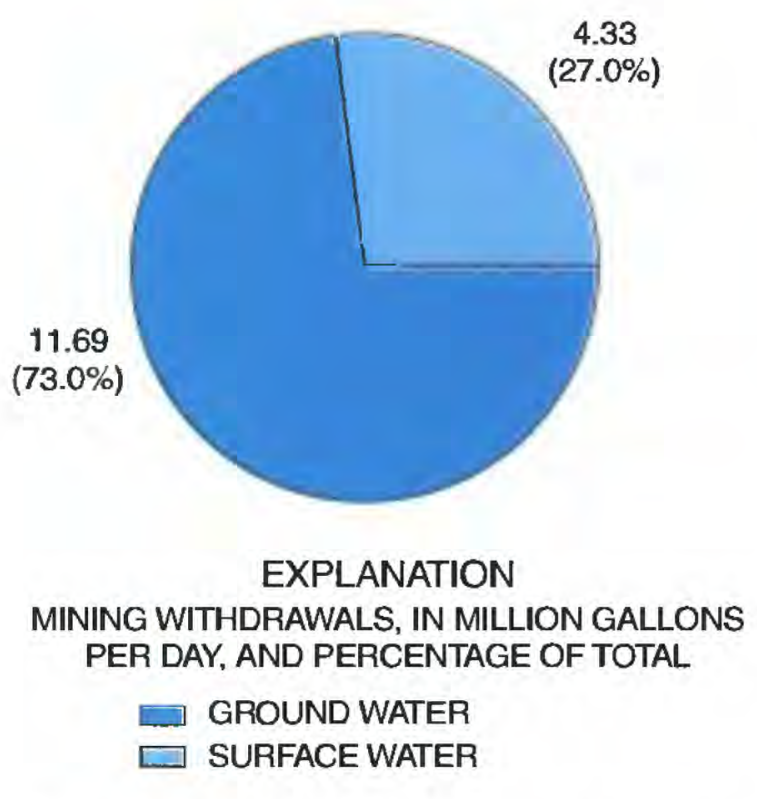

Figure 16. Source of water for mining use in North Carolina, 1995.

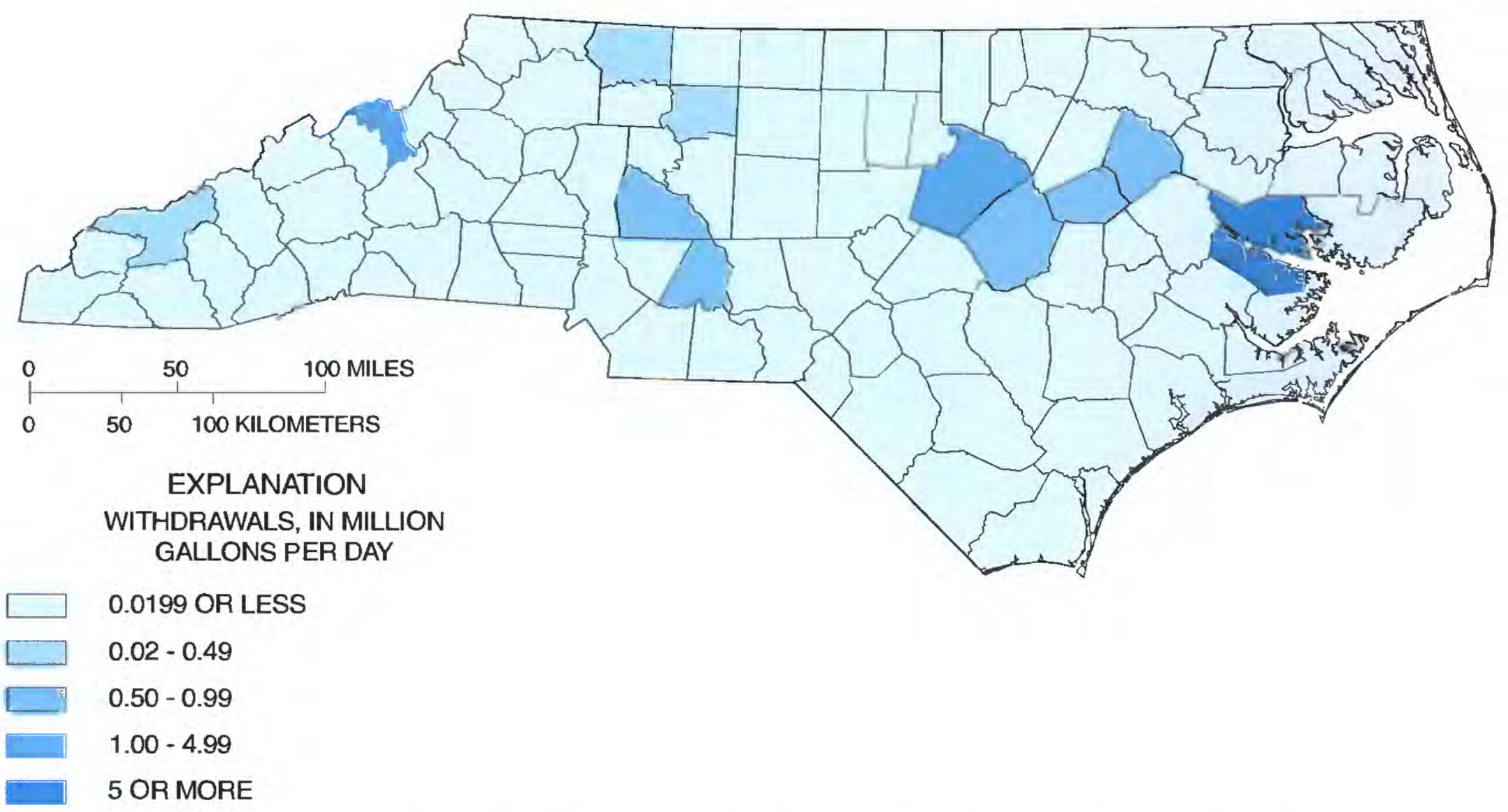

Figure 17. Total withdrawals for mining water use, by county, in North Carolina, 1995. (Data from the U.S. Geological Survey National Data Storage and Retrieval System.) 
Table 9. Mining water use, in million gallons per day, by county, in North Carolina, 1995

\begin{tabular}{|c|c|c|c|c|}
\hline County & $\begin{array}{l}\text { Ground } \\
\text { water }\end{array}$ & $\begin{array}{l}\text { Surtace } \\
\text { water }\end{array}$ & Total & $\begin{array}{c}\text { Consumptive } \\
\text { use }\end{array}$ \\
\hline ALAMANCE & 0.00 & 0.00 & 0.00 & 0.00 \\
\hline ALEXANDER & 0.00 & 0.00 & 0.00 & 0.00 \\
\hline ALLEGHANY & 0.00 & 0.00 & 0.00 & 0.00 \\
\hline ANSON & 0.00 & 0.00 & 0.00 & 0.00 \\
\hline ASHE & 0.00 & 0.00 & 0.00 & 0.00 \\
\hline AVERY & 0.00 & 0.00 & 0.00 & 0.00 \\
\hline BEAUFORT & 9.10 & 0.00 & 9.10 & 7.70 \\
\hline BERTIE & 0.00 & 0.00 & 0.00 & 0.00 \\
\hline BLADEN & 0.00 & 0.00 & 0.00 & 0.00 \\
\hline BRUNSWICK & 0.00 & 0.00 & 0.00 & 0.00 \\
\hline BUNCOMBE & 0.00 & 0.00 & 0.00 & 0.00 \\
\hline BURKE & 0.00 & 0.00 & 0.00 & 0.00 \\
\hline CABARRUS & 0.00 & 0.00 & 0.00 & 0.00 \\
\hline CALDWELL & 0.00 & 0.00 & 0.00 & 0.00 \\
\hline CAMDEN & 0.00 & 0.00 & 0.00 & 0.00 \\
\hline CARTERET & 0.00 & 0.00 & 0.00 & 0.00 \\
\hline CASWELL & 0.00 & 0.00 & 0.00 & 0.00 \\
\hline CATAWBA & 0.00 & 0.00 & 0.00 & 0.00 \\
\hline CHATHAM & 0.00 & 0.00 & 0.00 & 0.00 \\
\hline CHEROKEE & 0.00 & 0.00 & 0.00 & 0.00 \\
\hline CHOWAN & 0.00 & 0.00 & 0.00 & 0.00 \\
\hline CLAY & 0.00 & 0.00 & 0.00 & 0.00 \\
\hline CLEVELAND & 0.00 & 0.00 & 0.00 & 0.00 \\
\hline COLUMBUS & 0.00 & 0.00 & 0.00 & 0.00 \\
\hline CRAVEN & 0.00 & 0.00 & 0.00 & 0.00 \\
\hline CUMBERLAND & 0.00 & 0.00 & 0.00 & 0.00 \\
\hline CURRITUCK & 0.00 & 0.00 & 0.00 & 0.00 \\
\hline DARE & 0.00 & 0.00 & 0.00 & 0.00 \\
\hline DAVIDSON & 0.00 & 0.00 & 0.00 & 0.00 \\
\hline DAVIE & 0.01 & 0.00 & 0.01 & 0.01 \\
\hline DUPLIN & 0.00 & 0.00 & 0.00 & 0.00 \\
\hline DURHAM & 0.00 & 0.00 & 0.00 & 0.00 \\
\hline EDGECOMBE & 0.50 & 0.00 & 0.50 & 0.20 \\
\hline FORSYTH & 0.03 & 0.00 & 0.03 & 0.03 \\
\hline FRANKLIN & 0.00 & 0.00 & 0.00 & 0.00 \\
\hline GASTON & 0.00 & 0.00 & 0.00 & 0.00 \\
\hline GATES & 0.00 & 0.00 & 0.00 & 0.00 \\
\hline GRAHAM & 0.00 & 0.00 & 0.00 & 0.00 \\
\hline GRANVILLE & 0.00 & 0.00 & 0.00 & 0.00 \\
\hline GREENE & 0.00 & 0.00 & 0.00 & 0.00 \\
\hline GUILFORD & 0.00 & 0.00 & 0.00 & 0.00 \\
\hline HALIFAX & 0.00 & 0.00 & 0.00 & 0.00 \\
\hline HARNETT & 0.00 & 0.00 & 0.00 & 0.00 \\
\hline HAYWOOD & 0.00 & 0.00 & 0.00 & 0.00 \\
\hline HENDERSON & 0.00 & 0.00 & 0.00 & 0.00 \\
\hline HERTFORD & 0.00 & 0.00 & 0.00 & 0.00 \\
\hline HOKE & 0.00 & 0.00 & 0.00 & 0.00 \\
\hline HYDE & 0.00 & 0.00 & 0.00 & 0.00 \\
\hline IREDELL & 0.00 & 0.00 & 0.00 & 0.00 \\
\hline JACKSON & 0.00 & 0.00 & 0.00 & 0.00 \\
\hline
\end{tabular}


Table 9. Mining water use, in million gallons per day, by county, in North Carolina, 1995-Continued

\begin{tabular}{|c|c|c|c|c|}
\hline County & $\begin{array}{l}\text { Ground } \\
\text { water }\end{array}$ & $\begin{array}{l}\text { Surface } \\
\text { water }\end{array}$ & Total & $\begin{array}{c}\text { Consumptive } \\
\text { use }\end{array}$ \\
\hline JOHNSTON & 0.50 & 0.00 & 0.50 & 0.20 \\
\hline JONES & 0.00 & 0.00 & 0.00 & 0.00 \\
\hline LEE & 0.00 & 0.00 & 0.00 & 0.00 \\
\hline LENOIR & 0.00 & 0.00 & 0.00 & 0.00 \\
\hline LINCOLN & 0.00 & 0.00 & 0.00 & 0.00 \\
\hline MCDOWELL & 0.00 & 0.01 & 0.01 & 0.00 \\
\hline MACON & 0.00 & 0.00 & 0.00 & 0.00 \\
\hline MADISON & 0.00 & 0.00 & 0.00 & 0.00 \\
\hline MARTIN & 0.00 & 0.00 & 0.00 & 0.00 \\
\hline MECKLENBURG & 0.01 & 0.00 & 0.01 & 0.01 \\
\hline MITCHELL & 0.00 & 2.00 & 2.00 & 0.01 \\
\hline MONTGOMERY & 0.00 & 0.00 & 0.00 & 0.00 \\
\hline MOORE & 0.00 & 0.00 & 0.00 & 0.00 \\
\hline NASH & 0.00 & 0.00 & 0.00 & 0.00 \\
\hline NEW HANOVER & 0.00 & 0.00 & 0.00 & 0.00 \\
\hline NORTHAMPTON & 0.00 & 0.00 & 0.00 & 0.00 \\
\hline ONSLOW & 0.00 & 0.00 & 0.00 & 0.00 \\
\hline ORANGE & 0.00 & 0.00 & 0.00 & 0.00 \\
\hline PAMLICO & 0.00 & 0.00 & 0.00 & 0.00 \\
\hline PASQUOTANK & 0.00 & 0.00 & 0.00 & 0.00 \\
\hline PENDER & 0.00 & 0.00 & 0.00 & 0.00 \\
\hline PERQUIMANS & 0.00 & 0.00 & 0.00 & 0.00 \\
\hline PERSON & 0.00 & 0.00 & 0.00 & 0.00 \\
\hline PITT & 0.00 & 0.00 & 0.00 & 0.00 \\
\hline POLK & 0.00 & 0.00 & 0.00 & 0.00 \\
\hline RANDOLPH & 0.00 & 0.00 & 0.00 & 0.00 \\
\hline RICHMOND & 0.01 & 0.00 & 0.01 & 0.01 \\
\hline ROBESON & 0.00 & 0.00 & 0.00 & 0.00 \\
\hline ROCKINGHAM & 0.00 & 0.00 & 0.00 & 0.00 \\
\hline ROWAN & 0.02 & 0.86 & 0.88 & 0.14 \\
\hline RUTHERFORD & 0.00 & 0.00 & 0.00 & 0.00 \\
\hline SAMPSON & 0.00 & 0.00 & 0.00 & 0.00 \\
\hline SCOTLAND & 0.00 & 0.00 & 0.00 & 0.00 \\
\hline STANLY & 0.00 & 0.84 & 0.84 & 0.00 \\
\hline STOKES & 0.00 & 0.00 & 0.00 & 0.00 \\
\hline SURRY & 0.01 & 0.23 & 0.24 & 0.01 \\
\hline SWAIN & 0.00 & 0.39 & 0.39 & 0.39 \\
\hline TRANSYLVANIA & 0.00 & 0.00 & 0.00 & 0.00 \\
\hline TYRRELL & 0.00 & 0.00 & 0.00 & 0.00 \\
\hline UNION & 0.00 & 0.00 & 0.00 & 0.00 \\
\hline VANCE & 0.00 & 0.00 & 0.00 & 0.00 \\
\hline WAKE & 1.00 & 0.00 & 1.00 & 0.40 \\
\hline WARREN & 0.00 & 0.00 & 0.00 & 0.00 \\
\hline WASHINGTON & 0.00 & 0.00 & 0.00 & 0.00 \\
\hline WATAUGA & 0.00 & 0.00 & 0.00 & 0.00 \\
\hline WAYNE & 0.00 & 0.00 & 0.00 & 0.00 \\
\hline WILKES & 0.00 & 0.00 & 0.00 & 0.00 \\
\hline WILSON & 0.50 & 0.00 & 0.50 & 0.20 \\
\hline YADKIN & 0.00 & 0.00 & 0.00 & 0.00 \\
\hline YANCEY & 0.00 & 0.00 & 0.00 & 0.00 \\
\hline TOTAL & 11.69 & 4.33 & 16.02 & 9.31 \\
\hline
\end{tabular}




\section{Livestock Water Use}

The livestock category of water use is divided into two subcategories: (1) water associated with the production of "stock," such as beef and dairy cows, cattle, sheep, milk, poultry, and eggs; and (2) water associated with "animal specialties," such as horses and aquaculture of trout, bass, catfish, and crawfish. In most cases, the amount of water used for maintaining animals is relatively small compared to water used in other categories. Water used for all livestock was only 3 percent of all water withdrawn in North Carolina in 1995. Of the $297 \mathrm{Mgal} / \mathrm{d}$ used for all livestock, approximately 30 percent was from ground water (table 10; fig. 18).
Water used for stock accounted for 41 percent, or $121 \mathrm{Mgal} / \mathrm{d}$, of the water withdrawn for all livestock in the State in 1995 (table 10). Approximately 71 percent of the water stock was from ground water (fig. 18). Seven predominately rural counties, Chatham, Duplin, Moore, Randolph, Sampson, Union, and Wilkes Counties, had withdrawals of more than $5 \mathrm{Mgal} / \mathrm{d}$ for stock populations. Duplin County had the largest withdrawals for stock, $11 \mathrm{Mgal} / \mathrm{d}$ (fig. 19).

Withdrawals for the stock subcategory have shown substantial increases in the last decade and may be attributed to the increase in hog production in the State. The hog industry has historically been an important part of North Carolina agriculture. The
A.

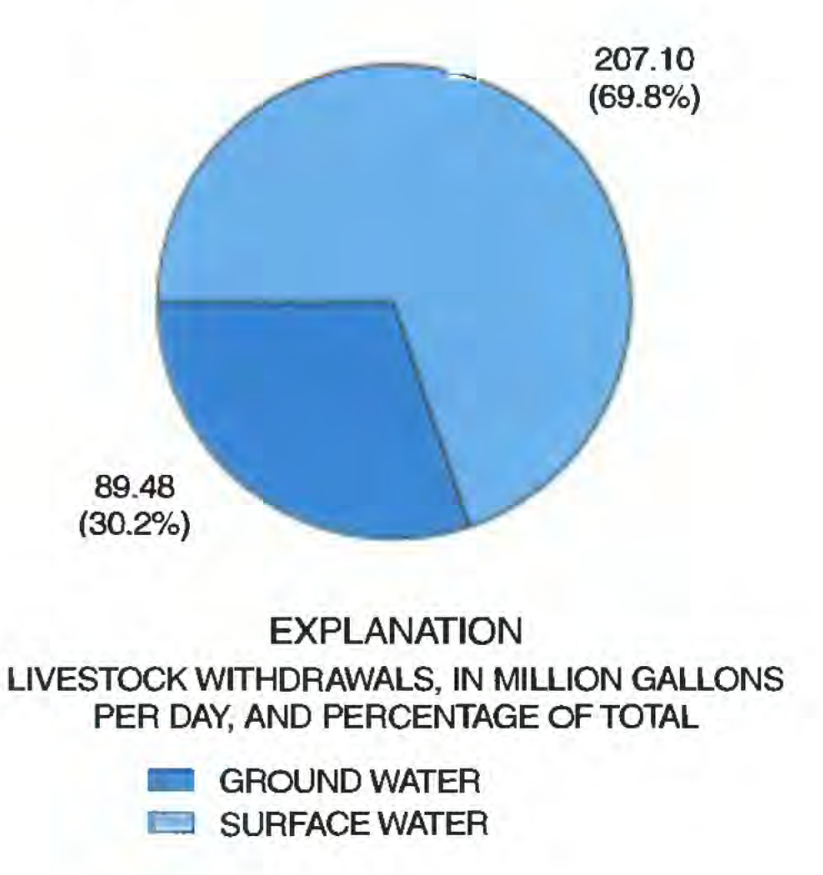

B.

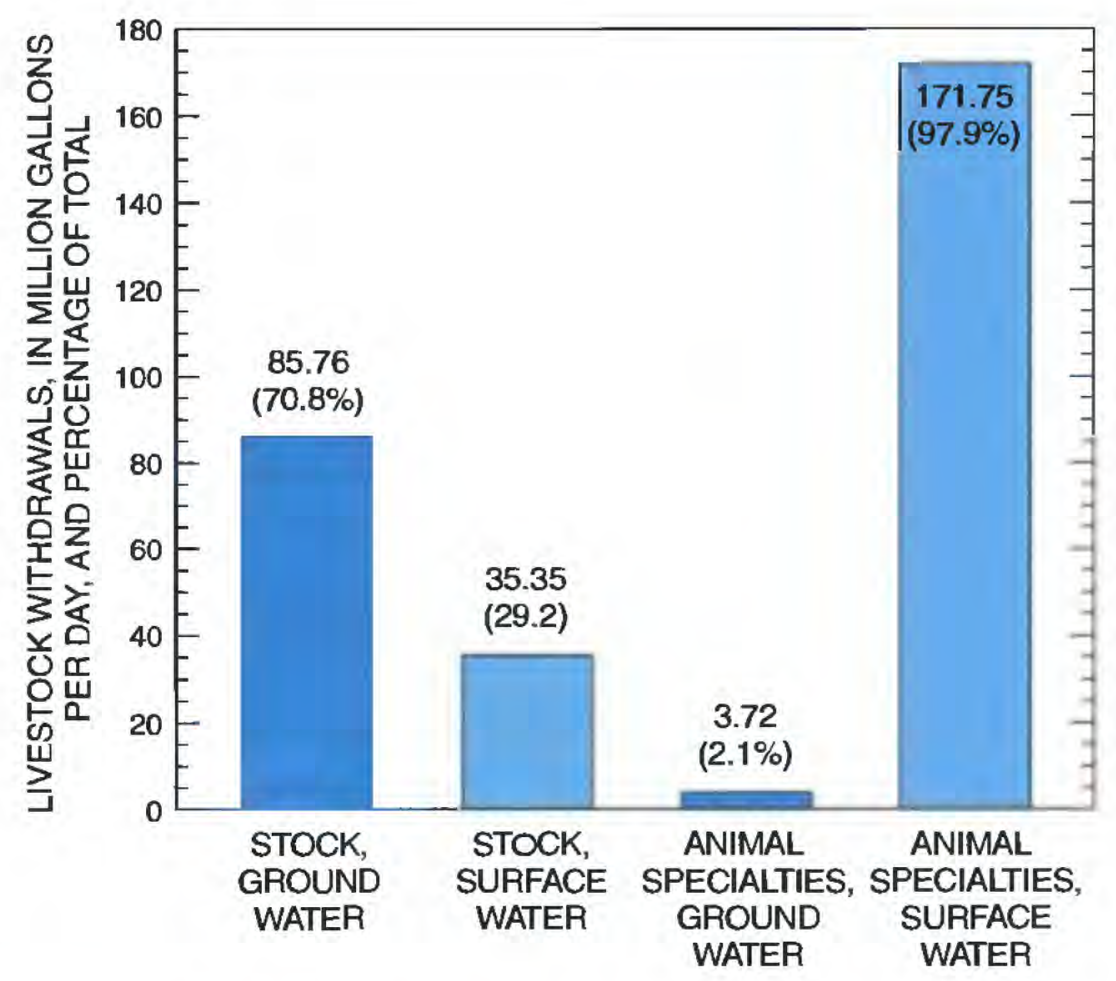

Figure 18. Source of water for livestock use in North Carolina, 1995. (A) Total and (B) Stock and animal specialties. 


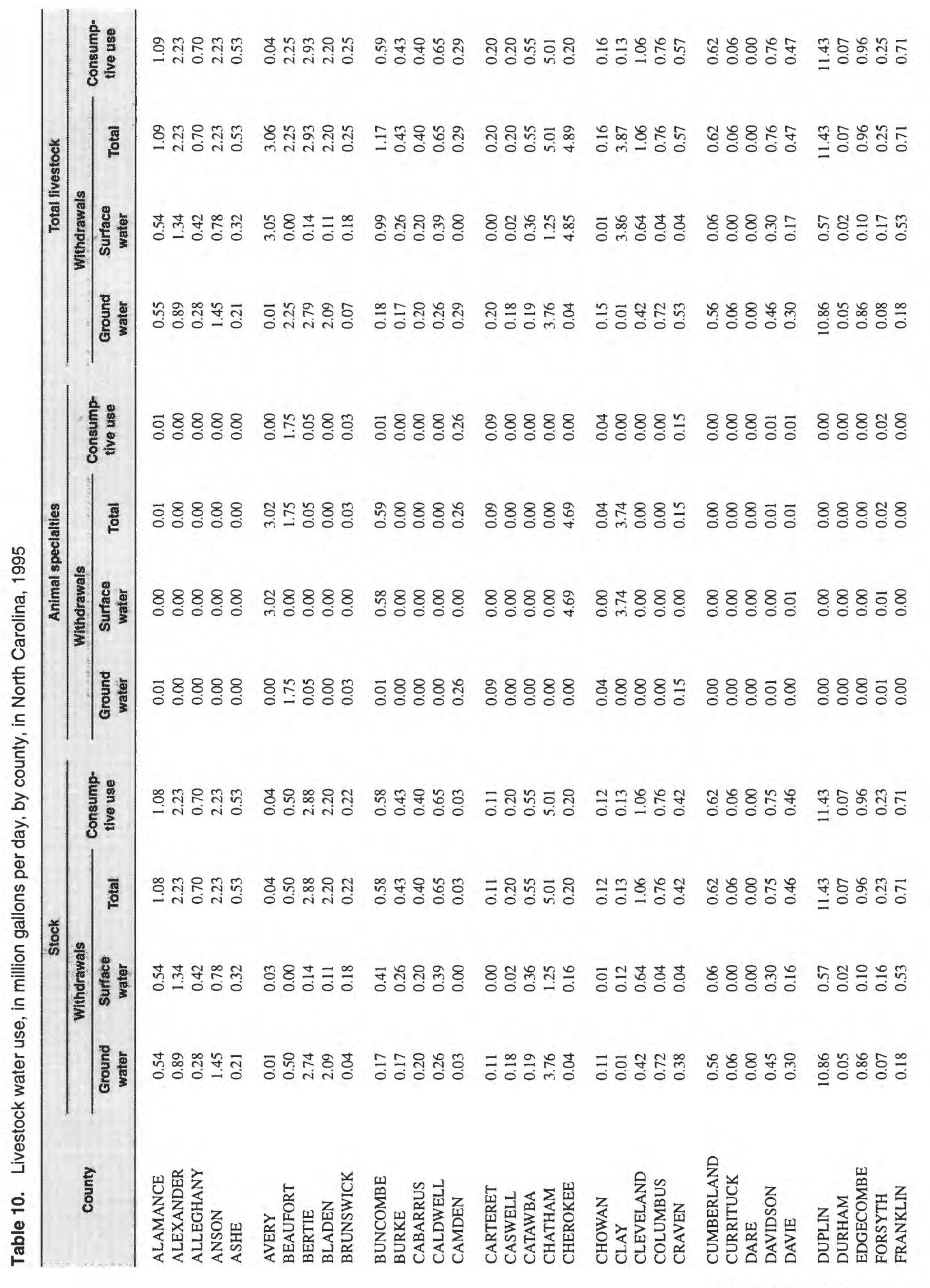




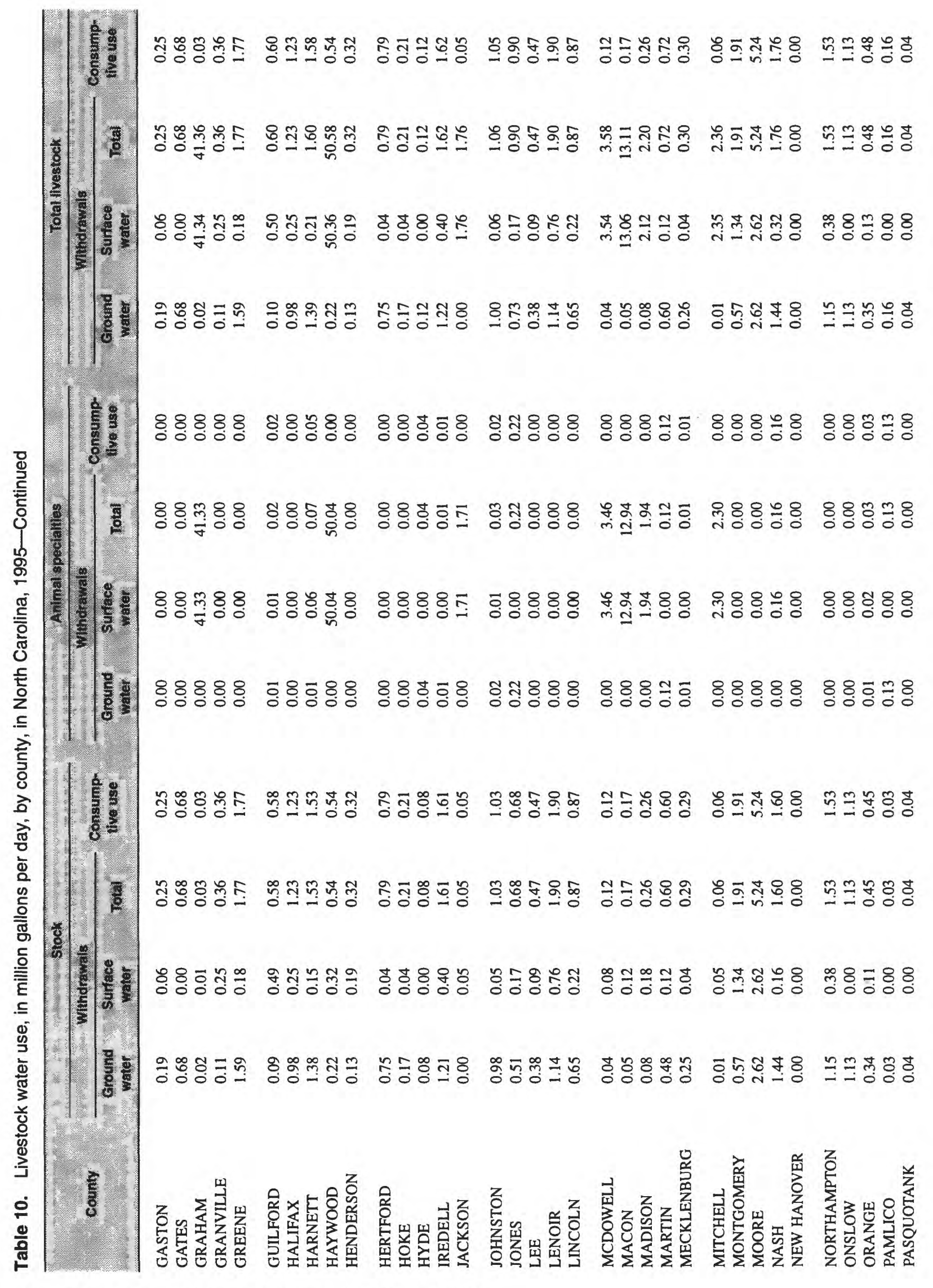




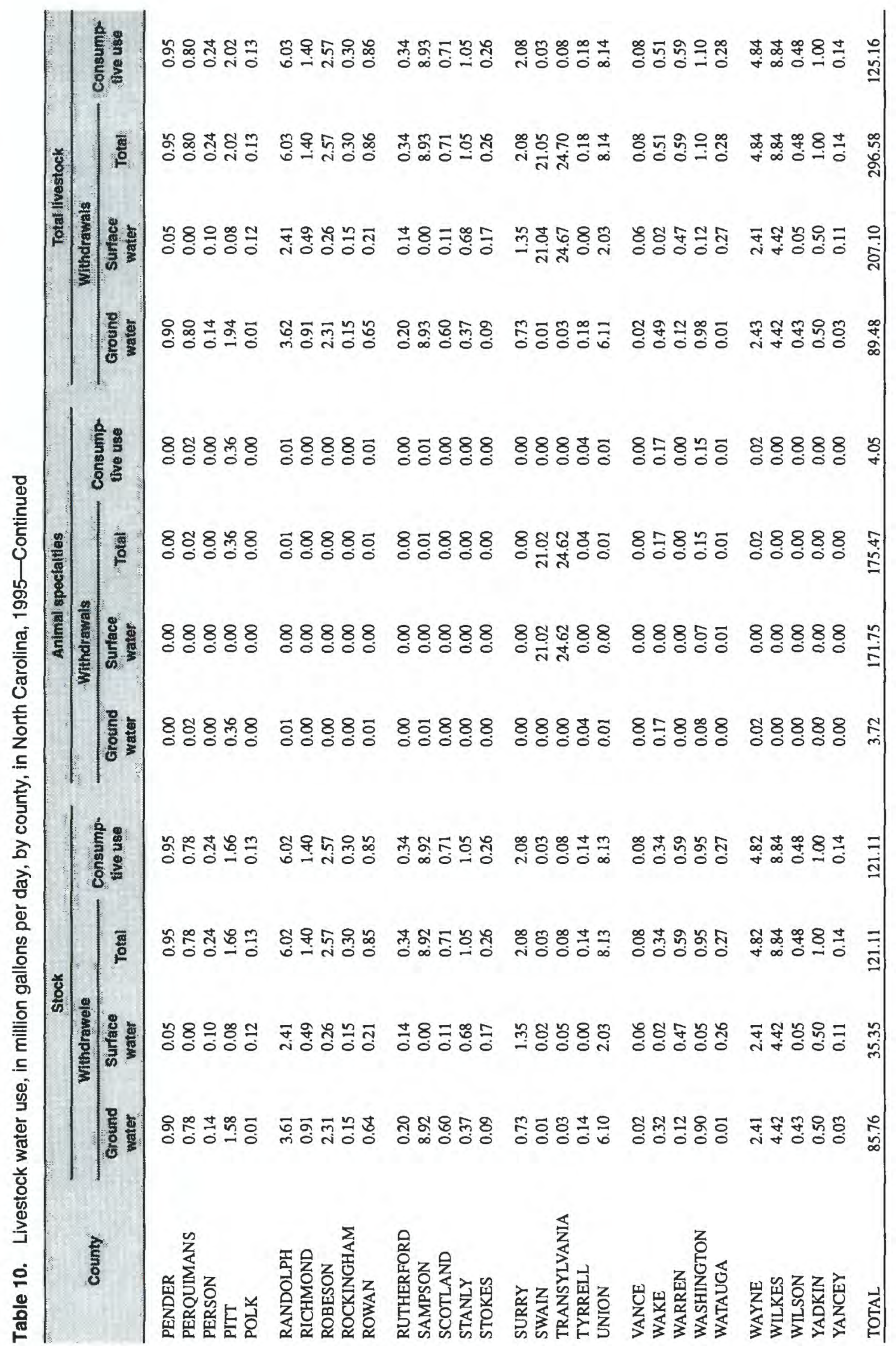




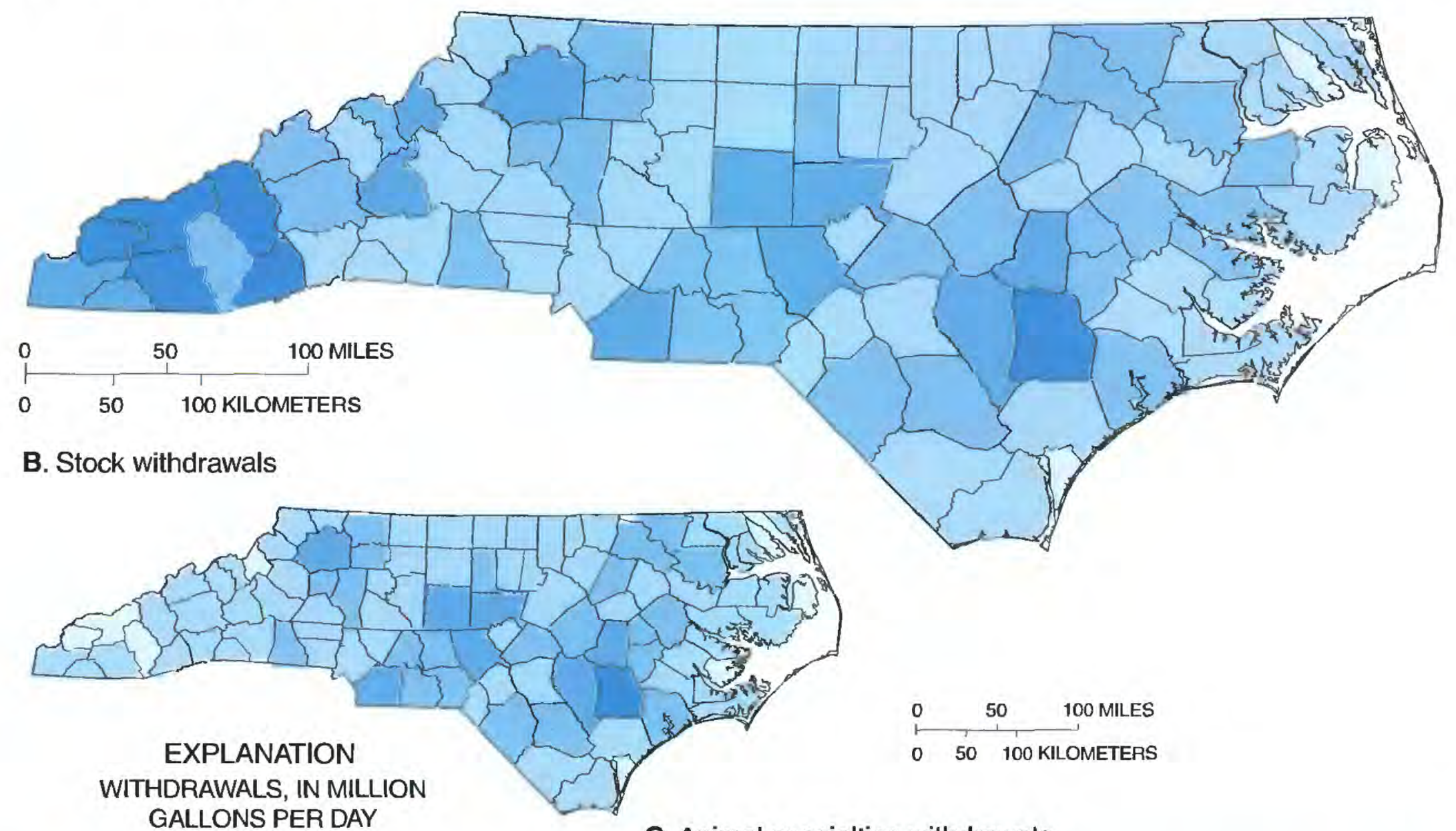

GALLONS PER DAY

0.0499 OR LESS

$0.05-0.99$

$1.00-2.99$

$3.00-9.99$

10 OR MORE

C. Animal specialties withdrawals

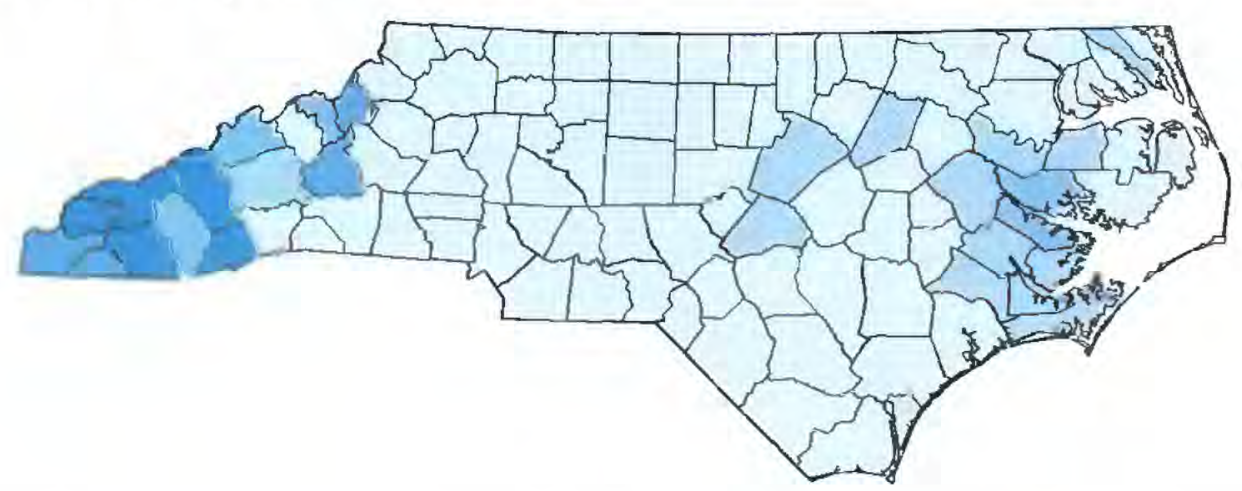

Figure 19. Livestock water use, by county, in North Carolina, 1995. (Data from the U.S. Geological Survey National Data Storage and Retrieval System.)

industry has changed from the small farms raising few hogs to the large confinement type operations of today. This shift is apparent when reviewing the change in the number and size of operations. North Carolina's hog population has increased almost 300 percent since 1991 (North Carolina Department of Agriculture, 1995a).

Animal specialties accounted for about 59 percent, or $176 \mathrm{Mgal} / \mathrm{d}$, of the livestock withdrawals in North Carolina in 1995 (table 10; fig. 18). About 98 percent of the water withdrawn for animal specialties was from surface water. Aquaculture accounted for more than 99 percent of the withdrawals for animal specialties. Aquaculture in the western part of the State is primarily trout farming. Because water is diverted from and returned to the original stream, consumptive use is minimal. Aquaculture in the eastern part of the State is primarily bass, catfish, and crawfish farming. Ground water is the source for about 92 percent of all the water used for eastern aquaculture.

\section{Irrigation Water Use}

In 1995, about $239 \mathrm{Mgal} / \mathrm{d}$ of water was used to irrigate more than 167,000 acres on crops, nurseries, and golf courses in the State (table 11). Seventy-six 
percent, or $181 \mathrm{Mgal} / \mathrm{d}$, of the withdrawals was from surface water (fig. 20). Four counties (Guilford, Mecklenburg, Moore, and Wake Counties) irrigated a total of 22,000 acres and had withdrawals of more than $10 \mathrm{Mgal} / \mathrm{d}$ each which accounted for 20 percent of all withdrawals for irrigation (table 11; fig. 21). Irrigation activities are influenced by the weather during the growing season. Because 1995 rainfall amounts were above average throughout the State, irrigation activities may have been less compared to years with average precipitation.

Crops and nurseries accounted for 72 percent, or 121,000 acres, of the irrigated acreage in the State. Land irrigated to grow tobacco accounted for 34 percent of this number. Crop and nursery irrigation accounted for about 40 percent, or $96 \mathrm{Mgal} / \mathrm{d}$, of the total withdrawals for irrigation (fig. 20). Surface water was the source for 76 percent, or $73 \mathrm{Mgal} / \mathrm{d}$, of these withdrawals.

A.

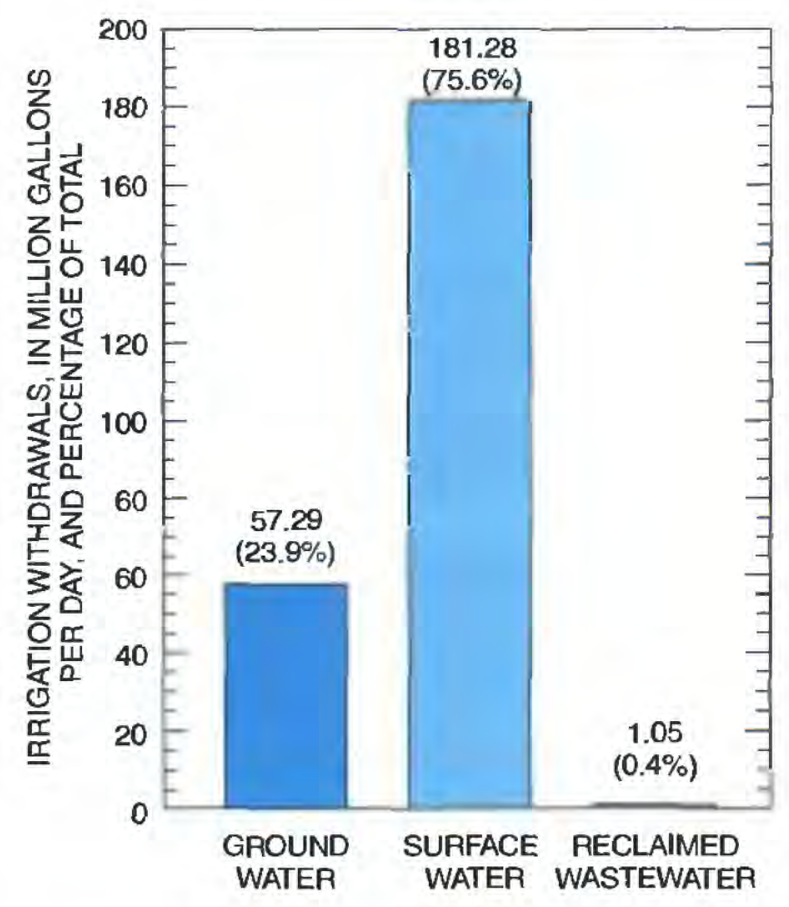

Golf is a popular recreational sport in North Carolina. There are approximately 495 public, private, and military golf courses across the State.

Approximately 46,000 acres were irrigated on golf courses, which was 38 percent of irrigated land in the State in 1995. Golf-course irrigation accounted for about 60 percent, or $144 \mathrm{Mgal} / \mathrm{d}$, of the total water withdrawals for irrigation in 1995 (fig. 20). About 76 percent of golf-course irrigation withdrawals was from surface water because most irrigation water comes from on-site runoff-collection ponds. However, during periods of below-average rainfall, ground water is sometimes used to maintain pond water levels.

Brunswick, Mecklenburg, and Wake Counties had the largest number of golf courses and withdrew more than 18 percent of all the withdrawals for golf course irrigation. A total of $1 \mathrm{Mgal} / \mathrm{d}$ of reclaimed wastewater was applied to golf courses in Brunswick, Carteret, Chatham, and Henderson Counties.
B.

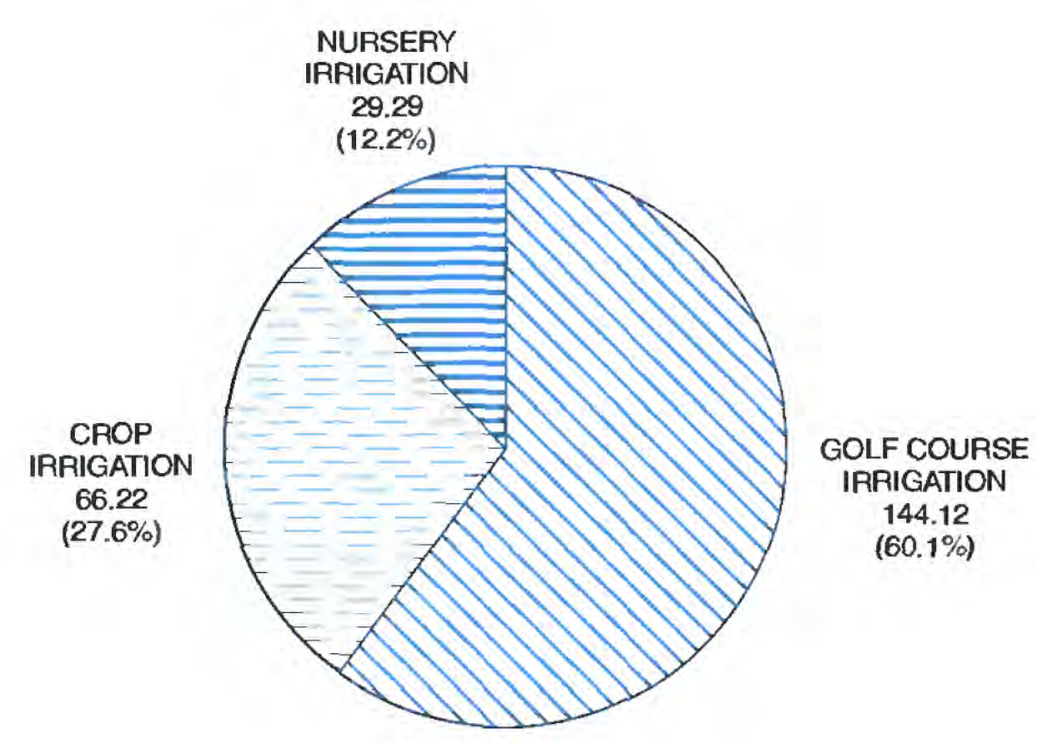

Figure 20. Irrigation water use, in million gallons per day, and percentage of total, by (A) source and (B) irrigation type, in North Carolina, 1995. 
Table 11. Irrigation water use, by county, in North Carolina, 1995

[Mgal/d, million gallons per day]

\begin{tabular}{|c|c|c|c|c|c|c|c|c|}
\hline \multirow[b]{2}{*}{ Counity } & \multicolumn{3}{|c|}{ Withdrawals, in Mgal/d } & \multirow{2}{*}{$\begin{array}{l}\text { Aeclaimad } \\
\text { wastewater, } \\
\text { In Mgalld }\end{array}$} & \multirow{2}{*}{$\begin{array}{l}\text { Consumptlve } \\
\text { use; } \\
\text { in Mgal/d }\end{array}$} & \multicolumn{3}{|c|}{ Irrigated land, by type, in thousand acre } \\
\hline & $\begin{array}{c}\text { Ground } \\
\text { water }\end{array}$ & $\begin{array}{l}\text { Surface } \\
\text { water }\end{array}$ & Total & & & Sprinkler & Drip & Total \\
\hline ALAMANCE & 0.00 & 3.81 & 3.81 & 0.00 & 3.81 & 2.12 & 0.00 & 2.12 \\
\hline ALEXANDER & 0.04 & 0.36 & 0.40 & 0.00 & 0.40 & 0.27 & 0.00 & 0.27 \\
\hline ALLEGHANY & 0.10 & 1.02 & 1.12 & 0.00 & 1.12 & 0.45 & 0.00 & 0.45 \\
\hline ANSON & 0.05 & 0.35 & 0.40 & 0.00 & 0.40 & 0.16 & 0.07 & 0.23 \\
\hline ASHE & 0.12 & 1.11 & 1.23 & 0.00 & 1.23 & 0.53 & 0.01 & 0.54 \\
\hline AVERY & 0.18 & 3.47 & 3.65 & 0.00 & 3.65 & 1.46 & 0.02 & 1.48 \\
\hline BEAUFORT & 0.68 & 0.17 & 0.85 & 0.00 & 0.85 & 0.55 & 0.01 & 0.56 \\
\hline BERTIE & 0.16 & 2.97 & 3.13 & 0.00 & 3.13 & 4.83 & 0.00 & 4.83 \\
\hline BLADEN & 0.41 & 0.95 & 1.36 & 0.00 & 1.36 & 1.22 & 0.02 & 1.24 \\
\hline BRUNSWICK & 1.61 & 6.65 & 8.26 & 0.31 & 8.26 & 2.77 & 0.00 & 2.77 \\
\hline BUNCOMBE & 0.09 & 2.09 & 2.18 & 0.00 & 2.18 & 1.43 & 0.01 & 1.44 \\
\hline BURKE & 0.13 & 2.55 & 2.68 & 0.00 & 2.68 & 1.28 & 0.05 & 1.33 \\
\hline CABARRUS & 0.11 & 1.43 & 1.54 & 0.00 & 1.54 & 0.66 & 0.02 & 0.68 \\
\hline CALDWELL & 0.32 & 3.06 & 3.38 & 0.00 & 3.38 & 1.70 & 0.02 & 1.72 \\
\hline CAMDEN & 0.02 & 0.02 & 0.04 & 0.00 & 0.04 & 0.07 & 0.00 & 0.07 \\
\hline CARTERET & 1.90 & 0.45 & 2.35 & 0.20 & 2.35 & 0.76 & 0.01 & 0.77 \\
\hline CASWELL & 1.55 & 0.00 & 1.55 & 0.00 & 1.55 & 2.15 & 0.00 & 2.15 \\
\hline CATAWBA & 0.72 & 2.82 & 3.54 & 0.00 & 3.54 & 1.32 & 0.10 & 1.42 \\
\hline CHATHAM & 0.05 & 0.39 & 0.44 & 0.30 & 0.44 & 0.44 & 0.19 & 0.63 \\
\hline CHEROKEE & 0.06 & 0.24 & 0.30 & 0.00 & 0.30 & 0.23 & 0.00 & 0.23 \\
\hline CHOWAN & 0.00 & 1.38 & 1.38 & 0.00 & 1.38 & 1.79 & 0.03 & 1.82 \\
\hline CLAY & 0.22 & 0.00 & 0.22 & 0.00 & 0.22 & 0.20 & 0.00 & 0.20 \\
\hline CLEVELAND & 0.25 & 2.16 & 2.41 & 0.00 & 2.41 & 1.13 & 0.01 & 1.14 \\
\hline COLUMBUS & 0.38 & 0.92 & 1.30 & 0.00 & 1.30 & 0.68 & $0.0 !$ & 0.69 \\
\hline CRAVEN & 2.31 & 0.58 & 2.89 & 0.00 & 2.89 & 1.23 & 0.01 & 1.24 \\
\hline CUMBERLAND & 0.92 & 3.66 & 4.58 & 0.00 & 4.58 & 3.12 & 0.04 & 3.16 \\
\hline CURRITUCK & 0.58 & 0.58 & 1.16 & 0.00 & 1.16 & 0.83 & 0.02 & 0.85 \\
\hline DARE & 1.31 & 0.30 & 1.61 & 0.00 & 1.61 & 0.45 & 0.00 & 0.45 \\
\hline DAVIDSON & 0.18 & 1.60 & 1.78 & 0.00 & 1.78 & 1.07 & 0.01 & 1.08 \\
\hline DAVIE & 0.56 & 1.27 & 1.83 & 0.00 & 1.83 & 0.75 & 0.03 & 0.78 \\
\hline DUPLIN & 1.06 & 2.49 & 3.55 & 0.00 & 3.55 & 3.49 & 0.06 & 3.55 \\
\hline DURHAM & 0.41 & 2.27 & 2.68 & 0.00 & 2.68 & 0.77 & 0.00 & 0.77 \\
\hline EDGECOMBE & 0.50 & 2.82 & 3.32 & 0.00 & 3.32 & 4.37 & 0.00 & 4.37 \\
\hline FORSYTH & 0.38 & 5.85 & 6.23 & 0.00 & 6.23 & 2.27 & 0.03 & 2.30 \\
\hline FRANKLIN & 0.09 & 3.99 & 4.08 & 0.00 & 4.08 & 3.85 & 0.04 & 3.89 \\
\hline GASTON & 0.35 & 1.91 & 2.26 & 0.00 & 2.26 & 0.77 & 0.00 & 0.77 \\
\hline GATES & 0.48 & 0.48 & 0.96 & 0.00 & 0.96 & 1.57 & 0.03 & 1.60 \\
\hline GRAHAM & 0.05 & 0.20 & 0.25 & 0.00 & 0.25 & 0.10 & 0.00 & 0.10 \\
\hline GRANVILLE & 0.23 & 2.07 & 2.30 & 0.00 & 2.30 & 3.08 & 0.06 & 3.14 \\
\hline GREENE & 0.30 & 0.46 & 0.76 & 0.00 & 0.76 & 1.26 & 0.00 & 1.26 \\
\hline GUILFORD & 1.06 & 9.59 & 10.65 & 0.00 & 10.65 & 5.30 & $0.3 i$ & 5.61 \\
\hline HALIFAX & 0.26 & 2.34 & 2.60 & 0.00 & 2.60 & 3.05 & 0.06 & 3.11 \\
\hline HARNETT & 0.59 & 2.33 & 2.92 & 0.00 & 2.92 & 3.08 & 0.06 & 3.14 \\
\hline HAYWOOD & 0.11 & 0.66 & 0.77 & 0.00 & 0.77 & 0.61 & 0.29 & 0.90 \\
\hline HENDERSON & 0.19 & 3.63 & 3.82 & 0.14 & 3.82 & 2.47 & 0.29 & 2.76 \\
\hline HERTFORD & 0.95 & 0.95 & 1.90 & 0.00 & 1.90 & 2.23 & 0.04 & 2.27 \\
\hline HOKE & 0.31 & 0.74 & 1.05 & 0.00 & 1.05 & 0.59 & 0.00 & 0.59 \\
\hline HYDE & 0.00 & 0.00 & 0.00 & 0.00 & 0.00 & 0.00 & 0.00 & 0.00 \\
\hline IREDELL & 0.25 & 2.17 & 2.42 & 0.00 & 2.42 & 1.30 & 0.04 & 1.34 \\
\hline JACKSON & 0.19 & 0.75 & 0.94 & 0.00 & 0.94 & 0.40 & 0.17 & 0.57 \\
\hline
\end{tabular}


Table 11. Irrigation water use, by county, in North Carolina, 1995-Continued

[Mgal/d. million gallons per day]

\begin{tabular}{|c|c|c|c|c|c|c|c|c|}
\hline \multirow[b]{2}{*}{ County } & \multicolumn{3}{|c|}{ Whithdrawals, in Mgat/d } & \multirow{2}{*}{$\begin{array}{l}\text { Reciaimed } \\
\text { westewater; } \\
\text { In Mgel/d }\end{array}$} & \multirow{2}{*}{$\begin{array}{c}\text { Consumptlve } \\
\text { use, } \\
\text { In Agal/d }\end{array}$} & \multicolumn{3}{|c|}{ Irrigatad land, by type, in thousand acres } \\
\hline & $\begin{array}{l}\text { Ground } \\
\text { water }\end{array}$ & $\begin{array}{c}\text { Surface } \\
\text { water }\end{array}$ & Total & & & Sprinkler & Drip & Totel \\
\hline JOHNSTON & 1.13 & 3.43 & 4.56 & 0.00 & 4.56 & 4.29 & 0.08 & 4.37 \\
\hline JONES & 0.50 & 0.13 & 0.63 & 0.00 & 0.63 & 0.61 & 0.01 & 0.62 \\
\hline LEE & 0.37 & 2.11 & 2.48 & 0.00 & 2.48 & 1.89 & 0.03 & 1.92 \\
\hline LENOIR & 0.52 & 0.94 & 1.46 & 0.00 & 1.46 & 1.06 & 0.02 & 1.08 \\
\hline LINCOLN & 0.08 & 0.70 & 0.78 & 0.00 & 0.78 & 0.44 & 0.01 & 0.45 \\
\hline MCDOWELL & 0.04 & 0.80 & 0.84 & 0.00 & 0.84 & 0.50 & 0.01 & 0.51 \\
\hline MACON & 0.04 & 1.00 & 1.04 & 0.00 & 1.04 & 0.58 & 0.08 & 0.66 \\
\hline MADISON & 0.11 & 0.40 & 0.51 & 0.00 & 0.51 & 0.38 & 0.01 & 0.39 \\
\hline MARTIN & 0.24 & 0.97 & 1.21 & 0.00 & 1.21 & 0.71 & 0.01 & 0.72 \\
\hline MECKLENBURG & 3.10 & 8.99 & 12.09 & 0.00 & 12.09 & 3.84 & 0.07 & 3.91 \\
\hline MITCHELL & 0.02 & 0.36 & 0.38 & 0.00 & 0.38 & 0.18 & 0.00 & 0.18 \\
\hline MONTGOMERY & 0.27 & 0.73 & 1.00 & 0.10 & 1.00 & 0.58 & 0.21 & 0.79 \\
\hline MOORE & 3.49 & 10.36 & 13.85 & 0.00 & 13.85 & 7.40 & 0.09 & 7.49 \\
\hline NASH & 0.58 & 5.23 & 5.81 & 0.00 & 5.81 & 7.68 & 0.15 & 7.83 \\
\hline NEW HANOVER & 1.90 & 1.78 & 3.68 & 0.00 & 3.68 & 1.13 & 0.04 & 1.17 \\
\hline NORTHAMPTON & 0.58 & 0.58 & 1.16 & 0.00 & 1.16 & 1.86 & 0.02 & 1.88 \\
\hline ONSLOW & 1.75 & 0.43 & 2.18 & 0.00 & 2.18 & 1.18 & 0.01 & 1.19 \\
\hline ORANGE & 0.76 & 2.28 & 3.04 & 0.00 & 3.04 & 1.64 & 0.02 & 1.66 \\
\hline PAMLICO & 1.84 & 0.00 & 1.84 & 0.00 & 1.84 & 2.23 & 0.00 & 2.23 \\
\hline PASQUOTANK & 0.33 & 0.33 & 0.66 & 0.00 & 0.66 & 0.19 & 0.00 & 0.19 \\
\hline PENDER & 0.54 & 1.60 & 2.14 & 0.00 & 2.14 & 0.94 & 0.04 & 0.98 \\
\hline PERQUIMANS & 0.22 & 0.09 & 0.31 & 0.00 & 0.31 & 0.50 & 0.03 & 0.53 \\
\hline PERSON & 0.17 & 1.56 & 1.73 & 0.00 & 1.73 & 2.39 & 0.05 & 2.44 \\
\hline PITT & 2.17 & 0.93 & 3.10 & 0.00 & 3.10 & 1.85 & 0.01 & 1.86 \\
\hline POLK & 0.03 & 0.57 & 0.60 & 0.00 & 0.60 & 0.27 & 0.07 & 0.34 \\
\hline RANDOLPH & 0.32 & 2.14 & 2.46 & 0.00 & 2.46 & 1.37 & 0.02 & 1.39 \\
\hline RICHMOND & 0.35 & 1.97 & 2.32 & 0.00 & 2.32 & 2.86 & 0.05 & 2.91 \\
\hline ROBESON & 0.87 & 2.08 & 2.95 & 0.00 & 2.95 & 2.25 & 0.04 & 2.29 \\
\hline ROCKINGHAM & 0.47 & 4.29 & 4.76 & 0.00 & 4.76 & 4.66 & 0.08 & 4.74 \\
\hline ROWAN & 0.18 & 1.58 & 1.76 & 0.00 & 1.76 & 1.16 & 0.02 & 1.18 \\
\hline RUTHERFORD & 0.12 & 2.33 & 2.45 & 0.00 & 2.45 & 1.02 & 0.00 & 1.02 \\
\hline SAMPSON & 1.16 & 4.62 & 5.78 & 0.00 & 5.78 & 8.06 & 0.41 & 8.47 \\
\hline SCOTLAND & 0.32 & 0.92 & 1.24 & 0.00 & 1.24 & 0.37 & 0.00 & 0.37 \\
\hline STANLY & 1.54 & 0.10 & 1.64 & 0.00 & 1.64 & 0.91 & 0.01 & 0.92 \\
\hline STOKES & 0.08 & 0.70 & 0.78 & 0.00 & 0.78 & 0.64 & 0.01 & 0.65 \\
\hline SURRY & 0.00 & 2.05 & 2.05 & 0.00 & 2.05 & 1.30 & 0.00 & 1.30 \\
\hline SWAIN & 0.02 & 0.10 & 0.12 & 0.00 & 0.12 & 0.11 & 0.00 & 0.11 \\
\hline TRANSYLVANIA & 0.38 & 1.66 & 2.04 & 0.00 & 2.04 & 0.87 & 0.00 & 0.87 \\
\hline TYRRELL & 0.00 & 0.00 & 0.00 & 0.00 & 0.00 & 0.00 & 0.00 & 0.00 \\
\hline UNION & 0.55 & 0.68 & 1.23 & 0.00 & 1.23 & 0.63 & 0.05 & 0.68 \\
\hline VANCE & 0.00 & 1.43 & 1.43 & 0.00 & 1.43 & 1.46 & 0.01 & 1.47 \\
\hline WAKE & 2.57 & 7.65 & 10.22 & 0.00 & 10.22 & 5.10 & 0.06 & 5.16 \\
\hline WARREN & 0.13 & 1.14 & 1.27 & 0.00 & 1.27 & 1.40 & 0.00 & 1.40 \\
\hline WASHINGTON & 1.01 & 0.34 & 1.35 & 0.00 & 1.35 & 2.02 & 0.04 & 2.06 \\
\hline WATAUGA & 0.07 & 1.40 & 1.47 & 0.00 & 1.47 & 0.61 & 0.00 & 0.61 \\
\hline WAYNE & 0.99 & 2.12 & 3.11 & 0.00 & 3.11 & 1.75 & 0.28 & 2.03 \\
\hline WILKES & 0.07 & 0.74 & 0.81 & 0.00 & 0.81 & 0.38 & 0.00 & 0.38 \\
\hline WILSON & 1.01 & 2.33 & 3.34 & 0.00 & 3.34 & 1.82 & 0.03 & 1.85 \\
\hline YADKIN & 1.35 & 0.01 & 1.36 & 0.00 & 1.36 & 1.30 & 0.01 & 1.31 \\
\hline YANCEY & 0.18 & 0.54 & 0.72 & 0.00 & 0.72 & 0.32 & 0.01 & 0.33 \\
\hline TOTAL & 57.29 & 181.28 & 238.57 & 1.05 & 238.57 & 162.90 & 4.37 & 167.27 \\
\hline
\end{tabular}




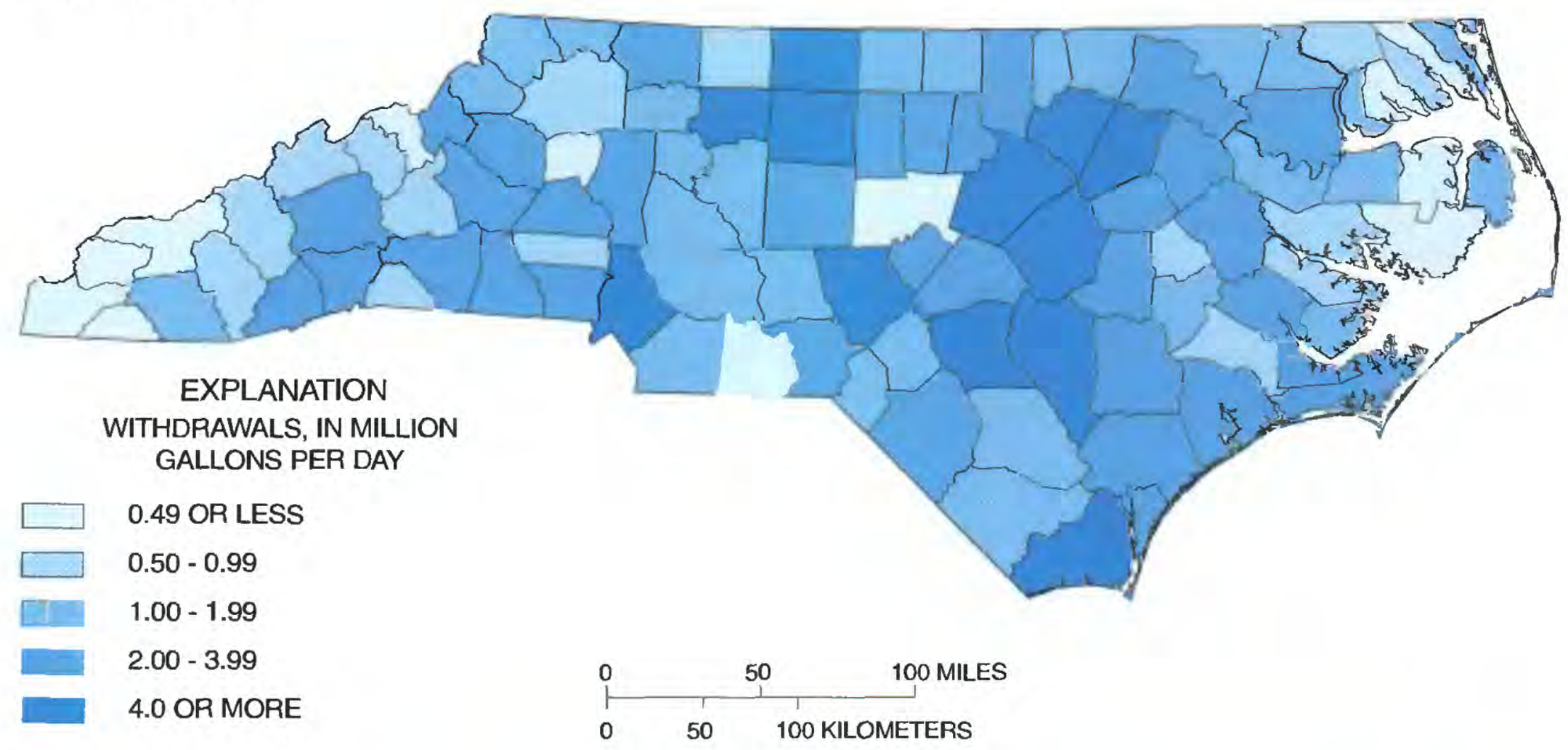

B. Total acres irrigated

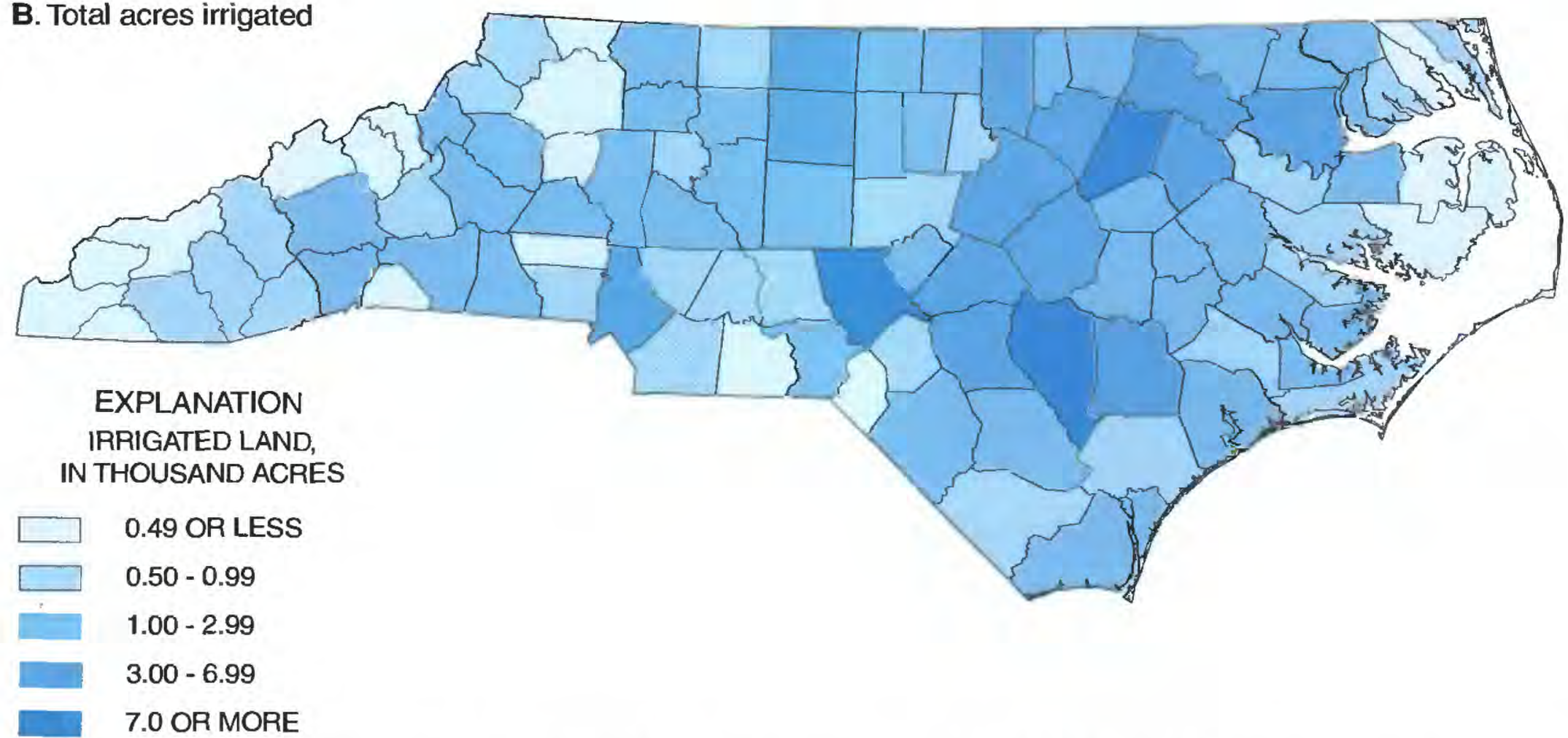

Figure 21. Irrigation water use, by county, in North Carolina, 1995. (Data from the U.S. Geological Survey National Data Storage and Retrieval System.)

\section{Thermoelectric-Power Generation Water Use}

Thermoelectric-power generation includes water use associated with the generation of power by fossil fuel and nuclear energy. Thermoelectric-power generation facilities are the largest water users in the State. These facilities withdrew 80 percent, or
7,417 Mgal/d, of the total (table 12). North Carolina had 21 thermoelectric powerplants operating in 1995; 18 fossil-fuel facilities withdrew about 43 percent, or $3,207 \mathrm{Mgal} / \mathrm{d}$, of the thermoelectric withdrawals. Nuclear facilities in Mecklenburg and Wake Counties withdrew 36 percent, or 2,656 Mgal/d, of the 


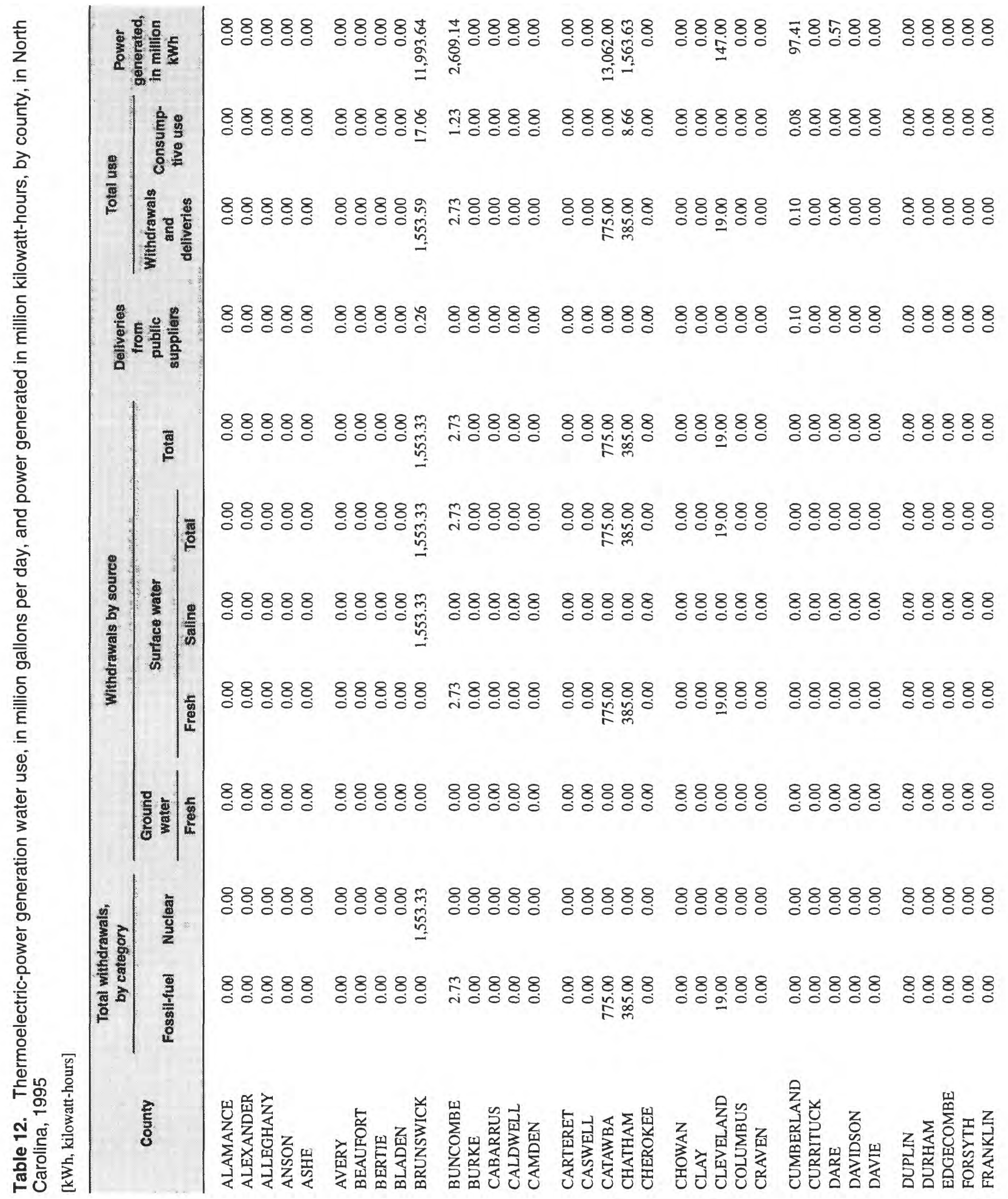




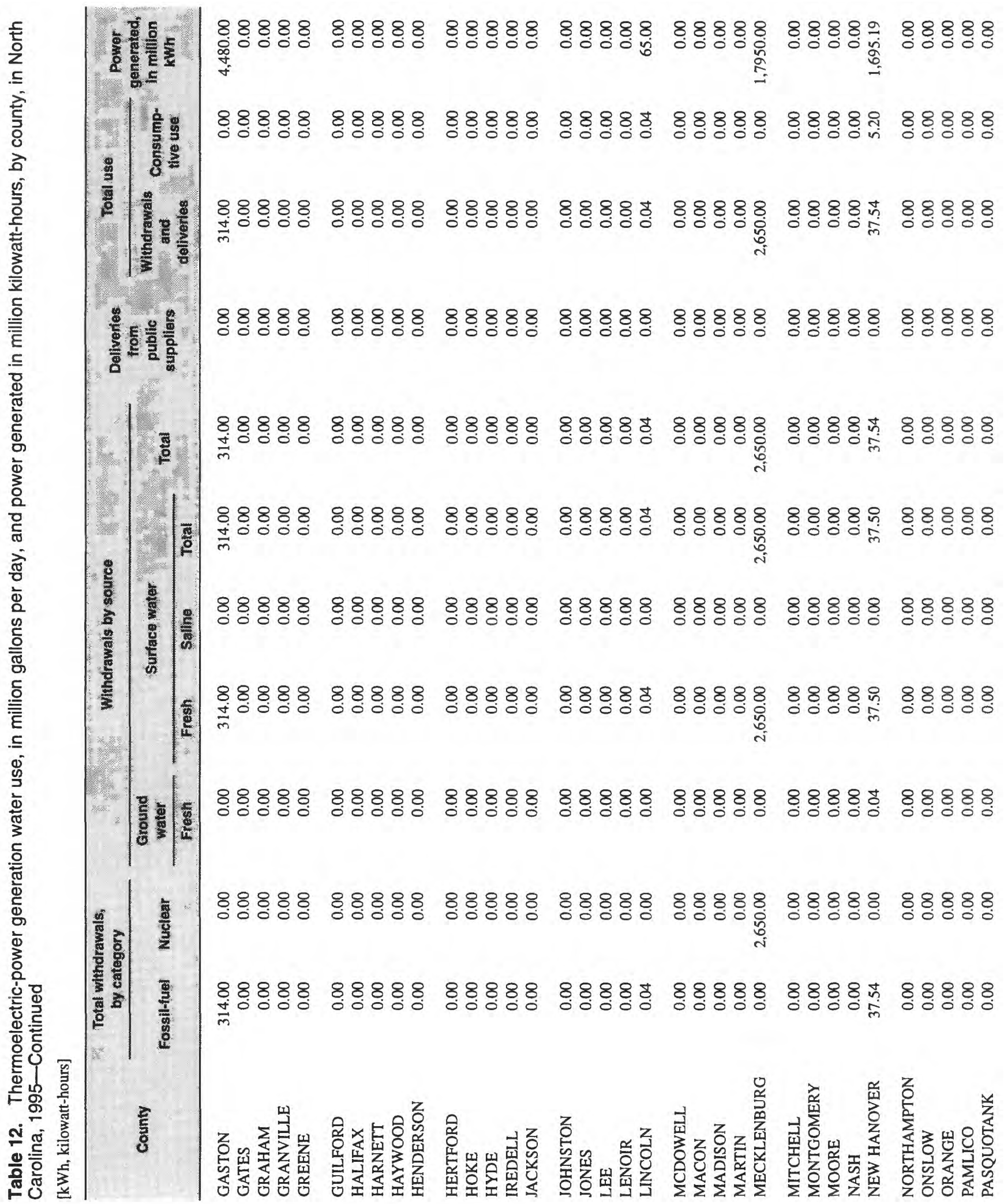




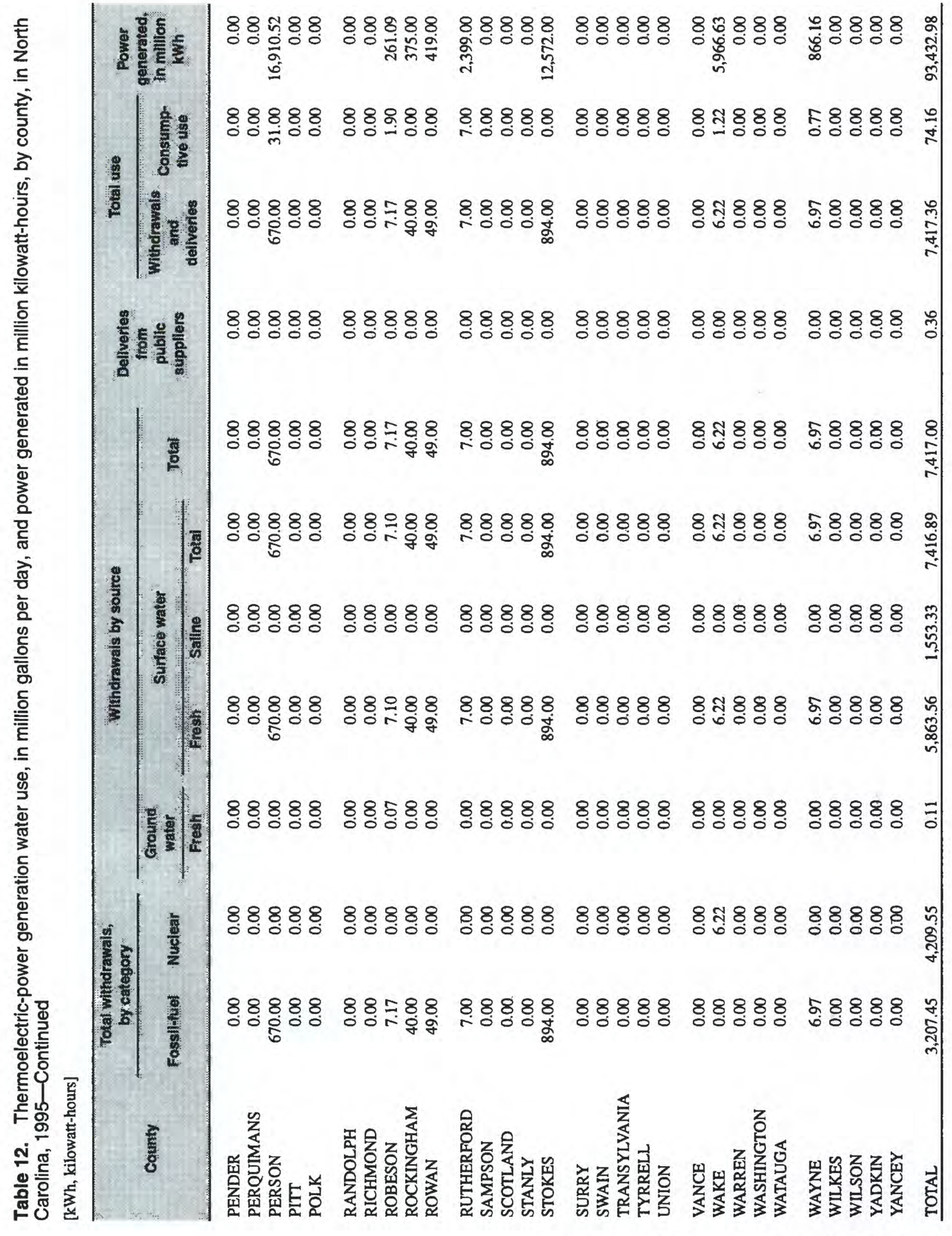


thermoelectric withdrawals (figs. 22 and 23). A nuclear facility in Brunswick County withdrew 1,553 Mgal/d of saline water from the Cape Fear estuary for cooling. This amount is 21 percent of the total water used for this category. Of the water withdrawn, 99 percent was returned to surface water.

The water source for thermoelectric-power production was almost exclusively from surface water;
$0.11 \mathrm{Mgal} / \mathrm{d}$ was from ground water (table 12). Public suppliers delivered $0.36 \mathrm{Mgal} / \mathrm{d}$ to thermoelectric plants. Thermoelectric-powerplants produced about 93,433 million kilowatt-hours ( $\mathrm{kWh}$ ) of electricity in 1995. Fossil-fuel plants supplied 62 percent of this total, or 57,523 million kWh of electricity, and the remainder was produced by nuclear plants (table 12; fig. 23).

\section{A. Total withdrawals}

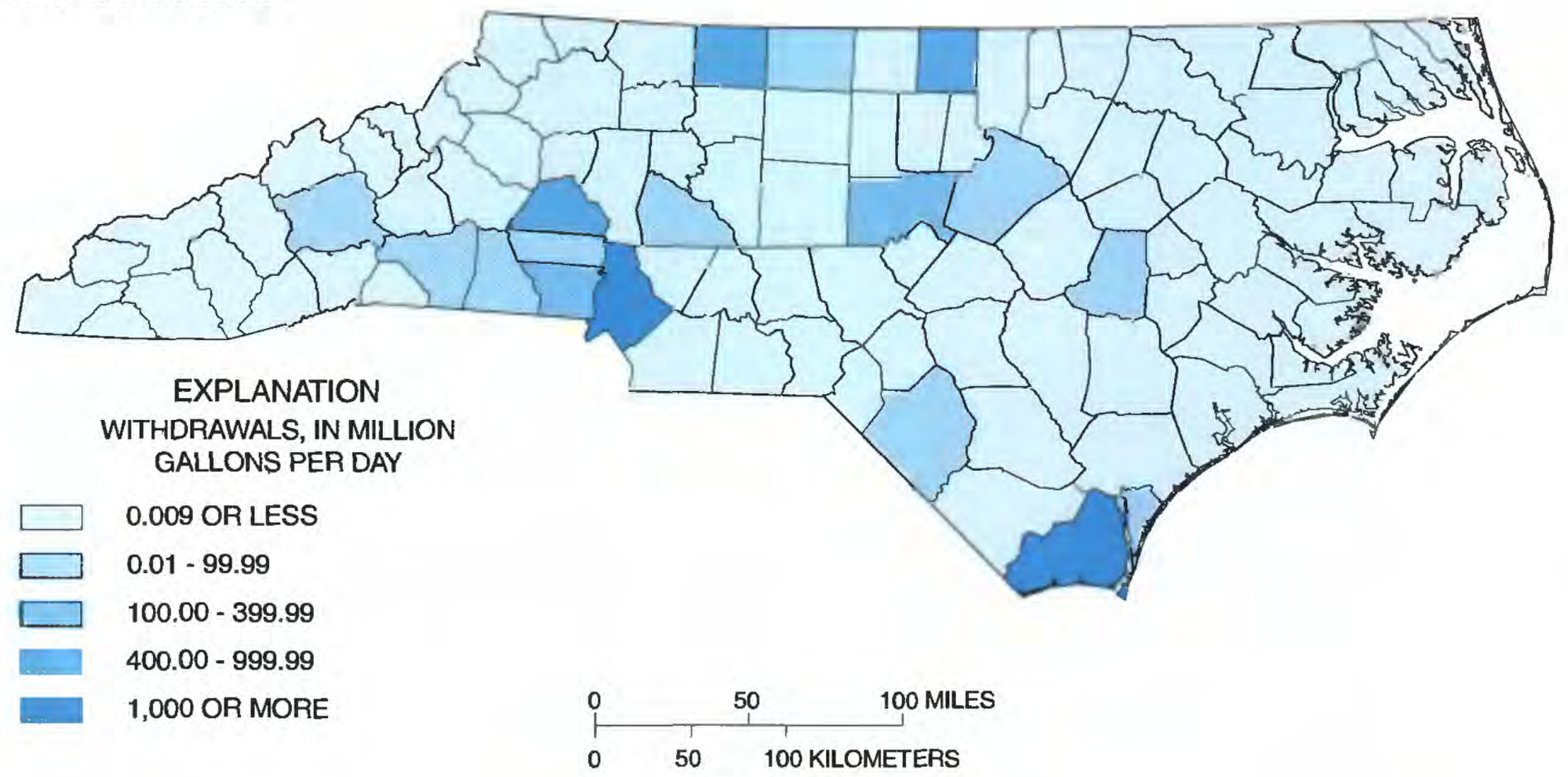

B. Total thermoelectric-power generation

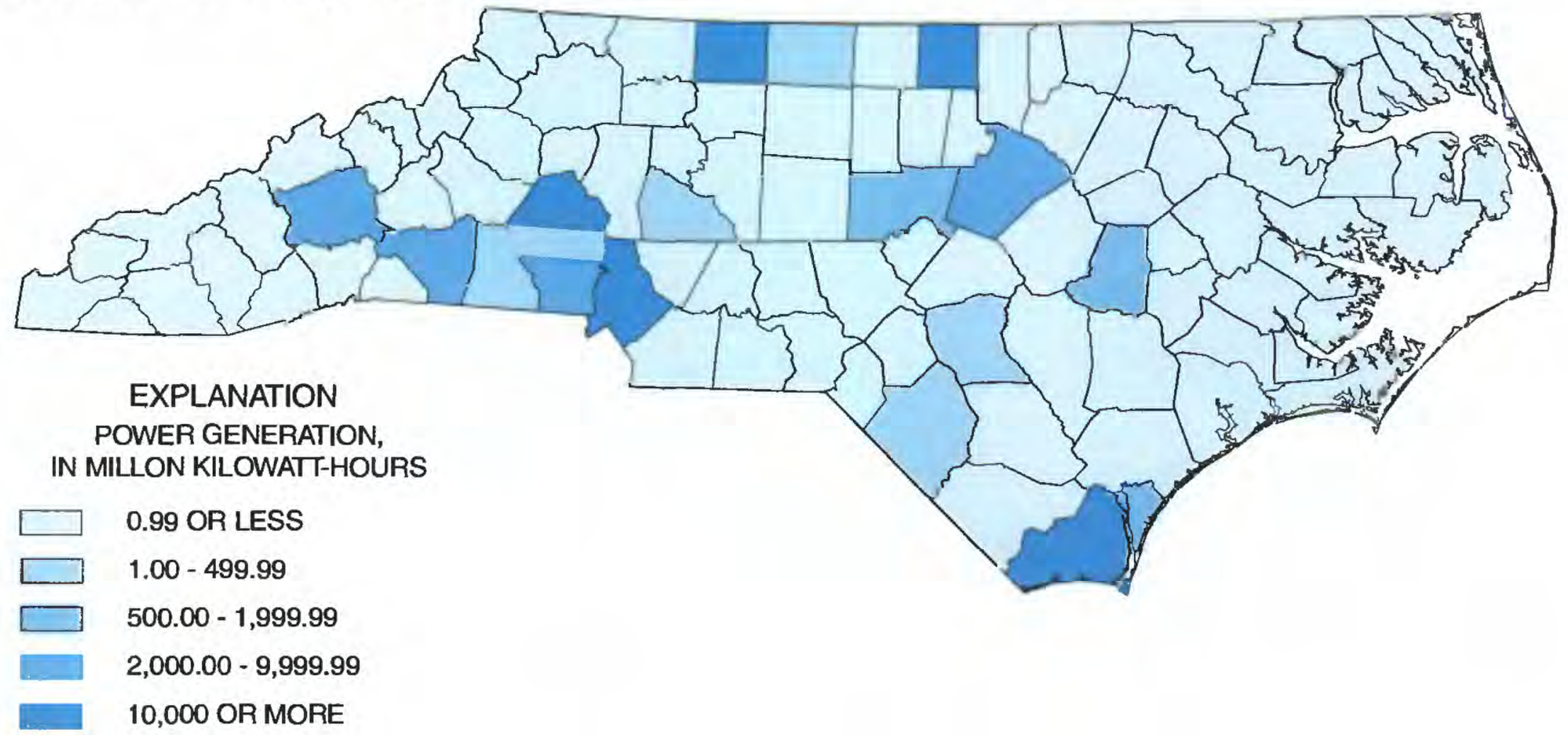

Figure 22. (A) Total water withdrawals for thermoelectric-power generation and (B) power generated, by county, in North Carolina, 1995. (Data from the U.S. Geological Survey National Data Storage and Retrieval System.) 
A.

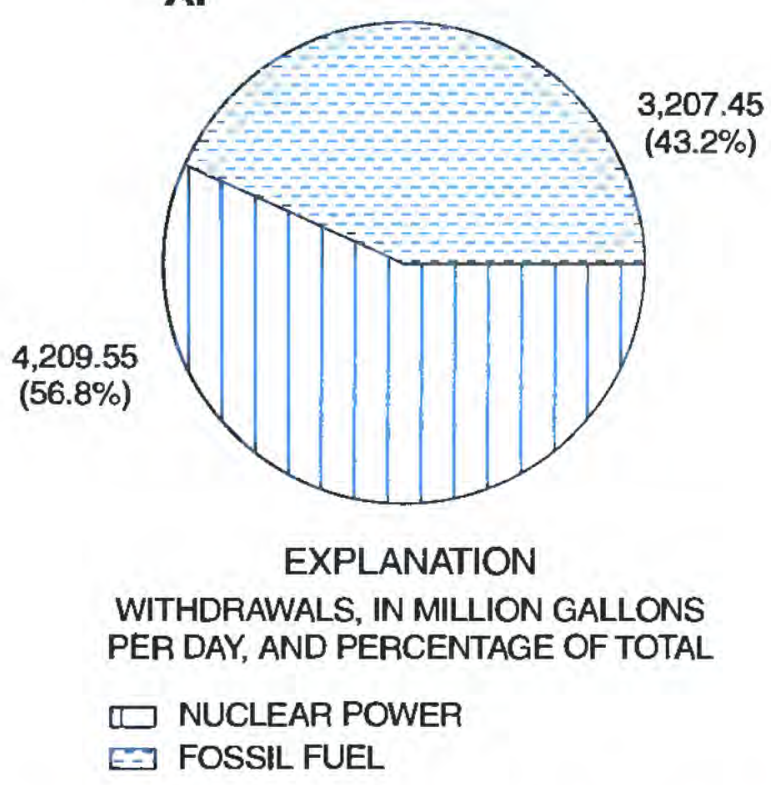

B.

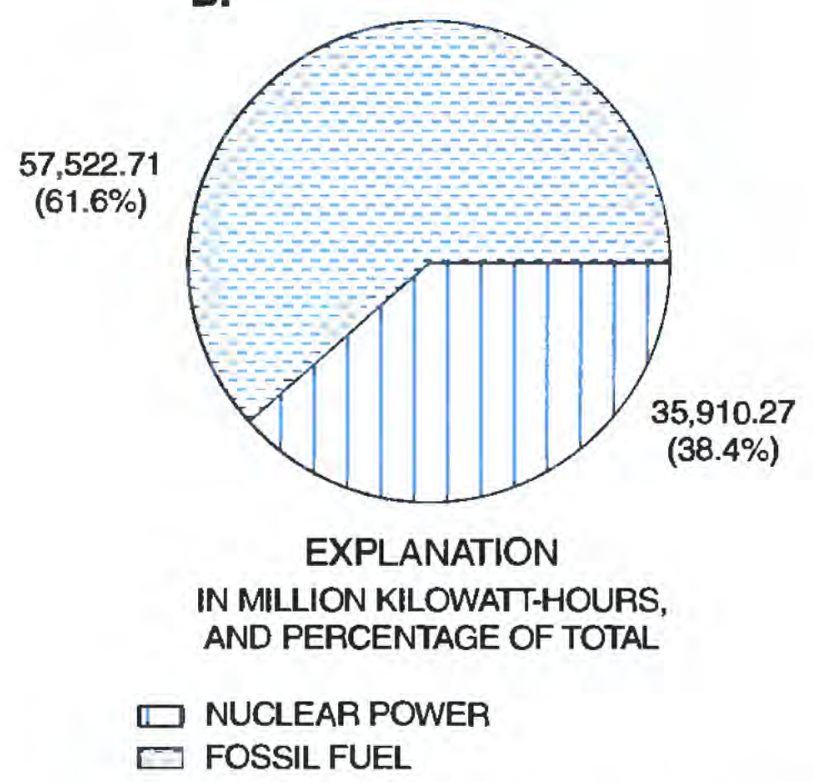

Figure 23. Thermoelectric-power generation, by power type, in North Carolina, 1995. (A) Water withdrawals and (B) Power generated.

\section{Instream Water Use for Hydroelectric- Power Generation}

In this report, instream water use is limited to water that is used in the generation of hydroelectric power. These data were collected from power companies that operate the 38 hydroelectric-power generation facilities in North Carolina. Even though water is not actually removed from the source, it is necessary to record the amount of water used by these facilities. Water budget planners must ensure that the amount of water needed to produce electricity is readily available to these facilities throughout the year, especially during drought conditions.

Hydroelectric-power generation occurs in the Blue Ridge and Piedmont Provinces of the State where topographic relief generally provides sufficient elevation drop essential for hydroelectric-power generation. Hydroelectric-power generation facilities require large amounts of water to drive turbines for the generation of electricity. The amount of water that passed through hydroelectric turbines in the State in 1995 was about $56,400 \mathrm{Mgal} / \mathrm{d}$ (table 13). This volume is more than six times the amount of all offstream water use in the State. Hydroelectric facilities in Halifax and Montgomery Counties, which have facilities located on major rivers, used 26,200 Mgal/d of water to produce electricity. This accounts for more than 46 percent of all water used by the 38 hydroelectric facilities in the State (table 13; fig. 24). Power generated by hydroelectric facilities totaled about 5,813 million $\mathrm{kWh}$ (table 13) or about 6 percent of all the electricity produced in the State. 
Table 13. Hydroelectric-power generation water use, by county, in North Carolina, 1995

[Mgal/d, million gallons per day; $\mathrm{kWh}$, kilowatt-hours]

\begin{tabular}{|c|c|c|}
\hline County & $\begin{array}{l}\text { Water uae, } \\
\text { in Mgal/d }\end{array}$ & $\begin{array}{l}\text { Power } \\
\text { generated, in } \\
\text { million kWh }\end{array}$ \\
\hline ALAMANCE & 0.00 & 0.00 \\
\hline ALEXANDER & 0.00 & 0.00 \\
\hline ALLEGHANY & 0.00 & 0.00 \\
\hline ANSON & $6,022.41$ & 151.30 \\
\hline ASHE & 0.00 & 0.00 \\
\hline AVERY & 0.00 & 0.00 \\
\hline BEAUFORT & 0.00 & 0.00 \\
\hline BERTIE & 0.00 & 0.00 \\
\hline BLADEN & 0.00 & 0.00 \\
\hline BRUNSWICK & 0.00 & 0.00 \\
\hline BUNCOMBE & 0.00 & 0.00 \\
\hline BURKE & 568.00 & 74.00 \\
\hline CABARRUS & 0.00 & 0.00 \\
\hline CALDWELL & $1,380.00$ & 80.00 \\
\hline CAMDEN & 0.00 & 0.00 \\
\hline CARTERET & 0.00 & 0.00 \\
\hline CASWELL & 0.00 & 0.00 \\
\hline CATAWBA & $1,400.00$ & 127.00 \\
\hline CHATHAM & 0.00 & 0.00 \\
\hline CHEROKEE & $1,441.29$ & 318.56 \\
\hline CHOWAN & 0.00 & 0.00 \\
\hline CLAY & 513.88 & 57.88 \\
\hline CLEVELAND & 164.00 & 2.00 \\
\hline COLUMBUS & 0.00 & 0.00 \\
\hline CRAVEN & 0.00 & 0.00 \\
\hline CUMBERLAND & 0.00 & 0.00 \\
\hline CURRITUCK & 0.00 & 0.00 \\
\hline DARE & 0.00 & 0.00 \\
\hline DAVIDSON & $3,113.72$ & 159.41 \\
\hline DAVIE & 0.00 & 0.00 \\
\hline DUPLIN & 0.00 & 0.00 \\
\hline DURHAM & 0.00 & 0.00 \\
\hline EDGECOMBE & 0.00 & 0.00 \\
\hline FORSYTH & 175.00 & 4.00 \\
\hline FRANKLIN & 0.00 & 0.00 \\
\hline GASTON & $1,773.00$ & 145.00 \\
\hline GATES & 0.00 & 0.00 \\
\hline GRAHAM & $6,079.30$ & $1,725.80$ \\
\hline GRANVILLE & 0.00 & 0.00 \\
\hline GREENE & 0.00 & 0.00 \\
\hline GUILFORD & 0.00 & 0.00 \\
\hline HALIFAX & $10,477.26$ & 646.51 \\
\hline HARNETT & 0.00 & 0.00 \\
\hline HAYWOOD & 499.66 & 399.05 \\
\hline HENDERSON & 72.00 & 23.00 \\
\hline HERTFORD & 0.00 & 0.00 \\
\hline HOKE & 0.00 & 0.00 \\
\hline HYDE & 0.00 & 0.00 \\
\hline IREDELL & $1,780.00$ & 111.00 \\
\hline JACKSON & 656.72 & 208.32 \\
\hline
\end{tabular}

Table 13. Hydroelectric-power generation water use, by county, in North Carolina, 1995-Continued

[Mgal/d, million gallons per day; $\mathrm{kWh}$, kilowatt-hours]

\begin{tabular}{|c|c|c|}
\hline County & $\begin{array}{l}\text { Water use, } \\
\text { in Mgal/d }\end{array}$ & $\begin{array}{l}\text { Power } \\
\text { generated; in } \\
\text { million kWh }\end{array}$ \\
\hline JOHNSTON & 0.00 & 0.00 \\
\hline JONES & 0.00 & 0.00 \\
\hline LEE & 0.00 & 0.00 \\
\hline LENOIR & 0.00 & 0.00 \\
\hline LINCOLN & $1,770.00$ & 205.00 \\
\hline MCDOWELL & 0.00 & 0.00 \\
\hline MACON & 742.02 & 213.28 \\
\hline MADISON & $1,615.00$ & 33.75 \\
\hline MARTIN & 0.00 & 0.00 \\
\hline MECKLENBURG & 0.00 & 0.00 \\
\hline MITCHELL & 0.00 & 0.00 \\
\hline MONTGOMERY & $15,707.24$ & $1,100.00$ \\
\hline MOORE & 0.00 & 0.00 \\
\hline NASH & 0.00 & 0.00 \\
\hline NEW HANOVER & 0.00 & 0.00 \\
\hline NORTHAMPTON & 0.00 & 0.00 \\
\hline ONSLOW & 0.00 & 0.00 \\
\hline ORANGE & 0.00 & 0.00 \\
\hline PAMLICO & 0.00 & 0.00 \\
\hline PASQUOTANK & 0.00 & 0.00 \\
\hline PENDER & 0.00 & 0.00 \\
\hline PERQUIMANS & 0.00 & 0.00 \\
\hline PERSON & 0.00 & 0.00 \\
\hline PITT & 0.00 & 0.00 \\
\hline POLK & 95.00 & 17.00 \\
\hline RANDOLPH & 0.00 & 0.00 \\
\hline RICHMOND & 0.00 & 0.00 \\
\hline ROBESON & 0.00 & 0.00 \\
\hline ROCKINGHAM & 0.00 & 0.00 \\
\hline ROWAN & 0.00 & 0.00 \\
\hline RUTHERFORD & 0.00 & 0.00 \\
\hline SAMPSON & 0.00 & 0.00 \\
\hline SCOTLAND & 0.00 & 0.00 \\
\hline STANLY & 0.00 & 0.00 \\
\hline STOKES & 0.00 & 0.00 \\
\hline SURRY & 0.00 & 0.00 \\
\hline SWAIN & 302.05 & 5.46 \\
\hline TRANSYLVANIA & 54.20 & 5.40 \\
\hline TYRRELL & 0.00 & 0.00 \\
\hline UNION & 0.00 & 0.00 \\
\hline VANCE & 0.00 & 0.00 \\
\hline WAKE & 0.00 & 0.00 \\
\hline WARREN & 0.00 & 0.00 \\
\hline WASHINGTON & 0.00 & 0.00 \\
\hline WATAUGA & 0.00 & 0.00 \\
\hline WAYNE & 0.00 & 0.00 \\
\hline WILKES & 0.00 & 0.00 \\
\hline WILSON & 0.00 & 0.00 \\
\hline YADKIN & 0.00 & 0.00 \\
\hline YANCEY & 0.00 & 0.00 \\
\hline TOTAL & $56,401.75$ & $5,812.72$ \\
\hline
\end{tabular}




\section{A. Total water use}

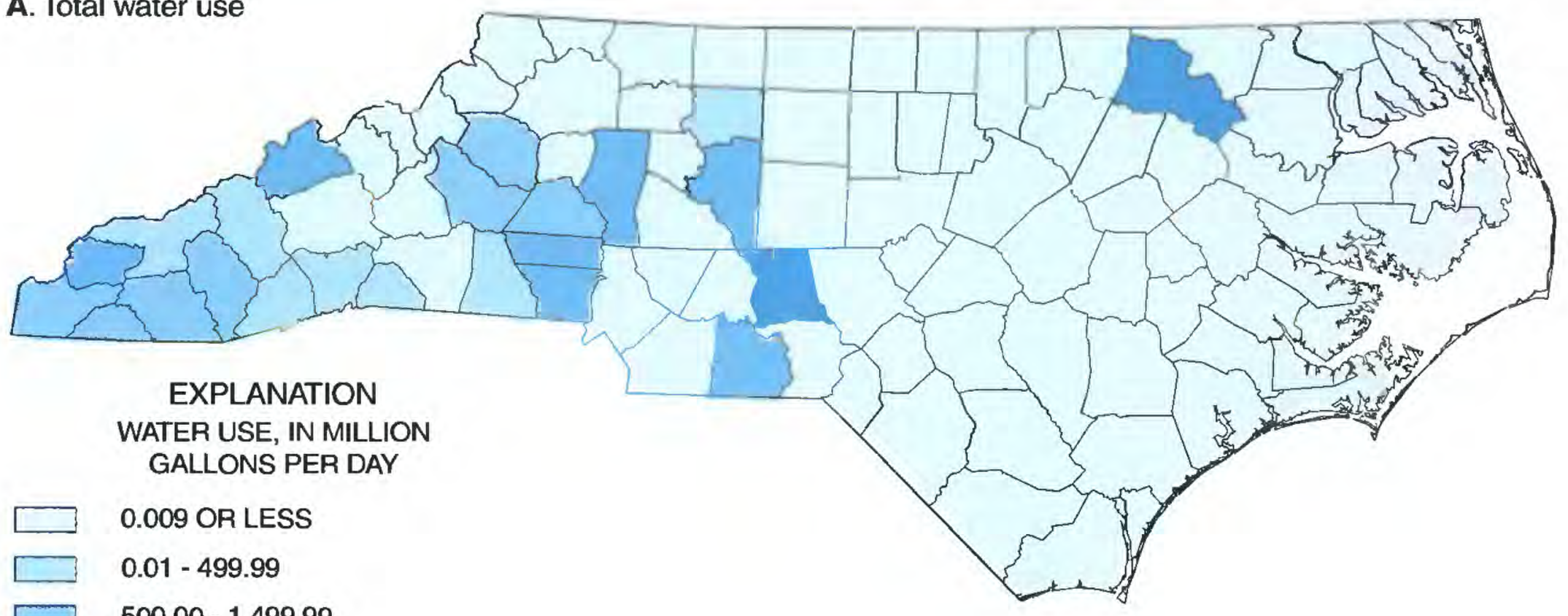

$500.00-1,499.99$

$1,500.00-9,999.99$

10,000 OR MORE

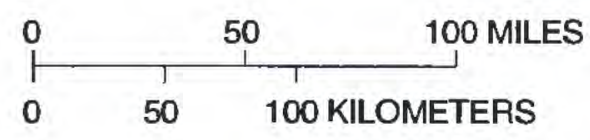

B. Total hydroelectric-power generation

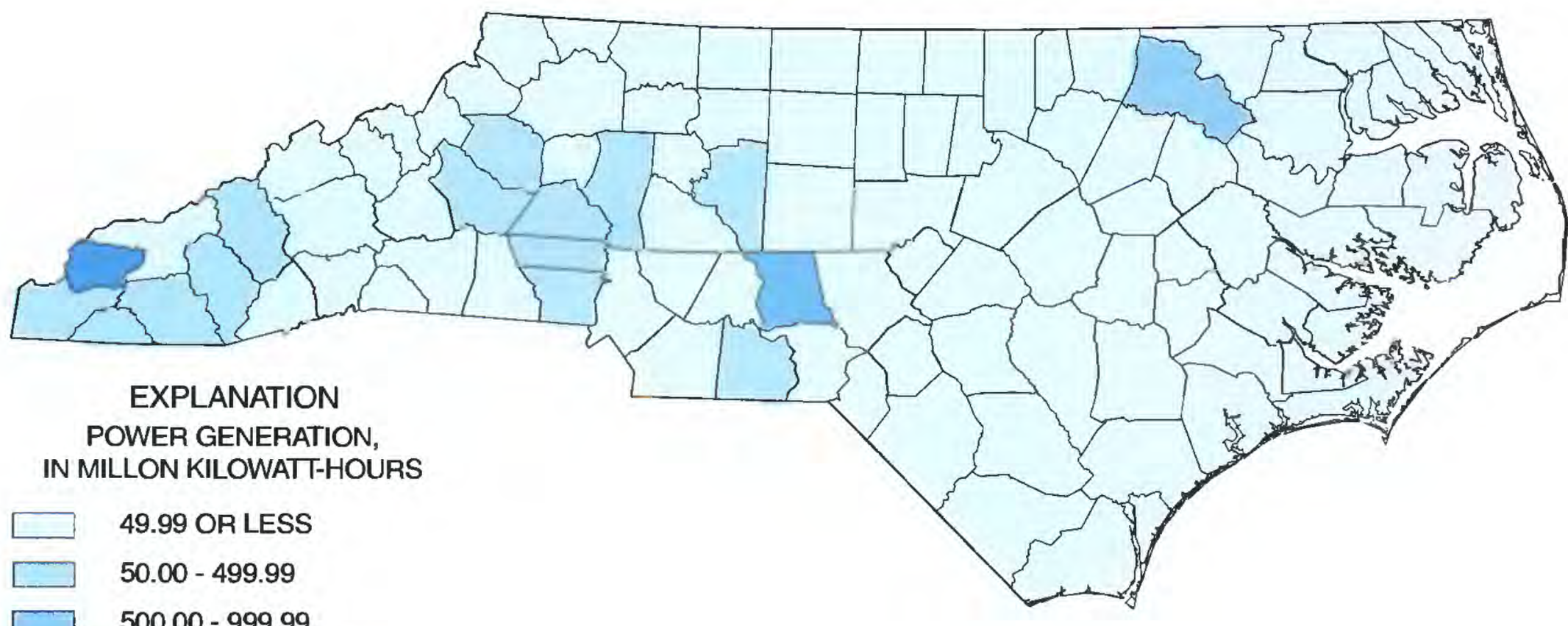

$500.00-999.99$

$1,000.00-1,499.99$

1,500 OR MORE

Figure 24. (A) Total instream water use for hydroelectric-power generation and (B) power generated, by county, in North Carolina, 1995. (Data from the U.S. Geological Survey National Data Storage and Retrieval System.) 


\section{SUMMARY}

In a cooperative effort between the U.S. Geological Survey and the Division of Water Resources of the North Carolina Department of Environment and Natural Resources, water use data for 1995 in North Carolina were collected for several categories. Data also were compiled from a number of other Federal, State, and private sources for the offstream water-use categories of public supply, domestic, commercial, industrial, mining, livestock, irrigation, and thermoelectric-power generation. Data for instream water use for hydroelectric-power generation were collected.

In 1995, an estimated 9,286 million gallons of water per day were withdrawn from surface- and ground-water sources in the State. Of this total, 94 percent came from surface water. Thermoelectricpower generation accounted for 80 percent of the total water withdrawn in the State, almost all of which was from surface water. Public-supply facilities delivered water to approximately 4.7 million consumers or 66 percent of the State's population. These facilities withdrew about $769 \mathrm{Mgal} / \mathrm{d}, 82$ percent of which was from surface water. Domestic users withdrew about $172 \mathrm{Mgal} / \mathrm{d}$, all from ground water. Commercial establishments withdrew only $7.60 \mathrm{Mgal} / \mathrm{d}$, mainly from ground water. Industries withdrew about $369 \mathrm{Mgal} / \mathrm{d}, 83$ percent of which was from surface water. Mining operations withdrew approximately $16 \mathrm{Mgal} / \mathrm{d}, 73$ percent of which was from ground water and does not include water withdrawn for dewatering of excavation pits. Water use for livestock was about $297 \mathrm{Mgal} / \mathrm{d}$; approximately 70 percent was from surface water. Water withdrawals to irrigate approximately 167,000 acres in the State was about $239 \mathrm{Mgal} / \mathrm{d} ; 76$ percent was from surface water. Thermoelectric-power generation facilities were the largest water users in the State and withdrew 80 percent, or $7,417 \mathrm{Mgal} / \mathrm{d}$, of all water withdrawn in North Carolina. Instream use for hydroelectricpower generation was about $56,400 \mathrm{Mgal} / \mathrm{d}$.

\section{SELECTED REFERENCES}

Crompton, E.J., and Solley, W.B., comps., 1995, Guidelines for preparing U.S. Geological Survey water-use estimates in the United States for 1995: U.S. Geological Survey home page on the World Wide Web, $<$ http://water.usgs.gov/public/watuse/guidelines/>.
Holland, T.W., 1992, Water-use data collection techniques in the Southeastern United States, Puerto Rico, and the U.S. Virgin Islands: U.S. Geological Survey WaterResources Investigations Report 92-4028, 76 p.

National Oceanic and Atmospheric Administration, 1996, Monthly and annual averages of temperature and precipitation: Asheville, N.C., Climatological Data Annual Summary, North Carolina, 1995 v. 100, p. 2-7 and reference notes.

North Carolina Department of Agriculture, 1995a, North Carolina agricultural statistics, 1995: Agricultural Statistics Division, North Carolina Department of Agriculture Number 180, 9-95-9000, 132 p.

$-1995 \mathrm{~b}$, Updated list of certified nurseries and plant collectors through September 30, 1995: Plant Industry Division, 149 p.

North Carolina Department of Commerce, 1995, North Carolina manufacturing firms, 1995 directory: Business/Industry Development Division.

North Carolina Department of Environment, Health, and Natural Resources, 1994, Permitted active and inactive mining operations in North Carolina as of June 1994: North Carolina Geological Survey, Land Quality Section, Division of Land Resources, North Carolina Geological Survey Open-File Report 94-4, 51 p.

Solley, W.B., Pierce, R.R., and Perlman, H.A., 1993, Estimated use of water in the United States in 1990: U.S. Geological Survey Circular 1081, 76 p.

Terziotti, Silvia, Schrader, T.P., and Treece, M.W., Jr., 1994, Estimated water use, by county, in North Carolina, 1990: U.S. Geological Survey Open-File Report 94-522, 102 p.

Treece, M.W., Jr., Bales, J.D., and Moreau, D.H., 1990, North Carolina water supply and use, in National Water Summary, 1987-Hydrologic events and water supply and use: U.S. Geological Survey Water-Supply Paper 2350 , p. 393-400.

U.S. Bureau of the Census, 1992, Census of agriculture, 1992: U.S. Department of Commerce, Economics and Statistics Administration, Bureau of the Census, September 1994, 556 p.

1996, Product announcement, CB 96-32: Population estimates for counties: Department of Commerce, U.S. Bureau of the Census, July 1, 1995, paperless product listing (PPL-39), on the World Wide Web, $<$ http://www.census.gov/>.

U.S. Department of Energy, Energy Information Administration, 1990, Monthly Power Plant Report, EIA-759.

Winner, M.D., Jr., and Simmons, C.E., 1977, Hydrology of the Creeping Swamp watershed, North Carolina, with reference to potential effects of stream channelization: U.S. Geological Survey Water-Resources Investigations Report 77-26, 54 p. 
APPENDI\& 
52 Estimated Water Use, by County, in North Carolina, 1995 


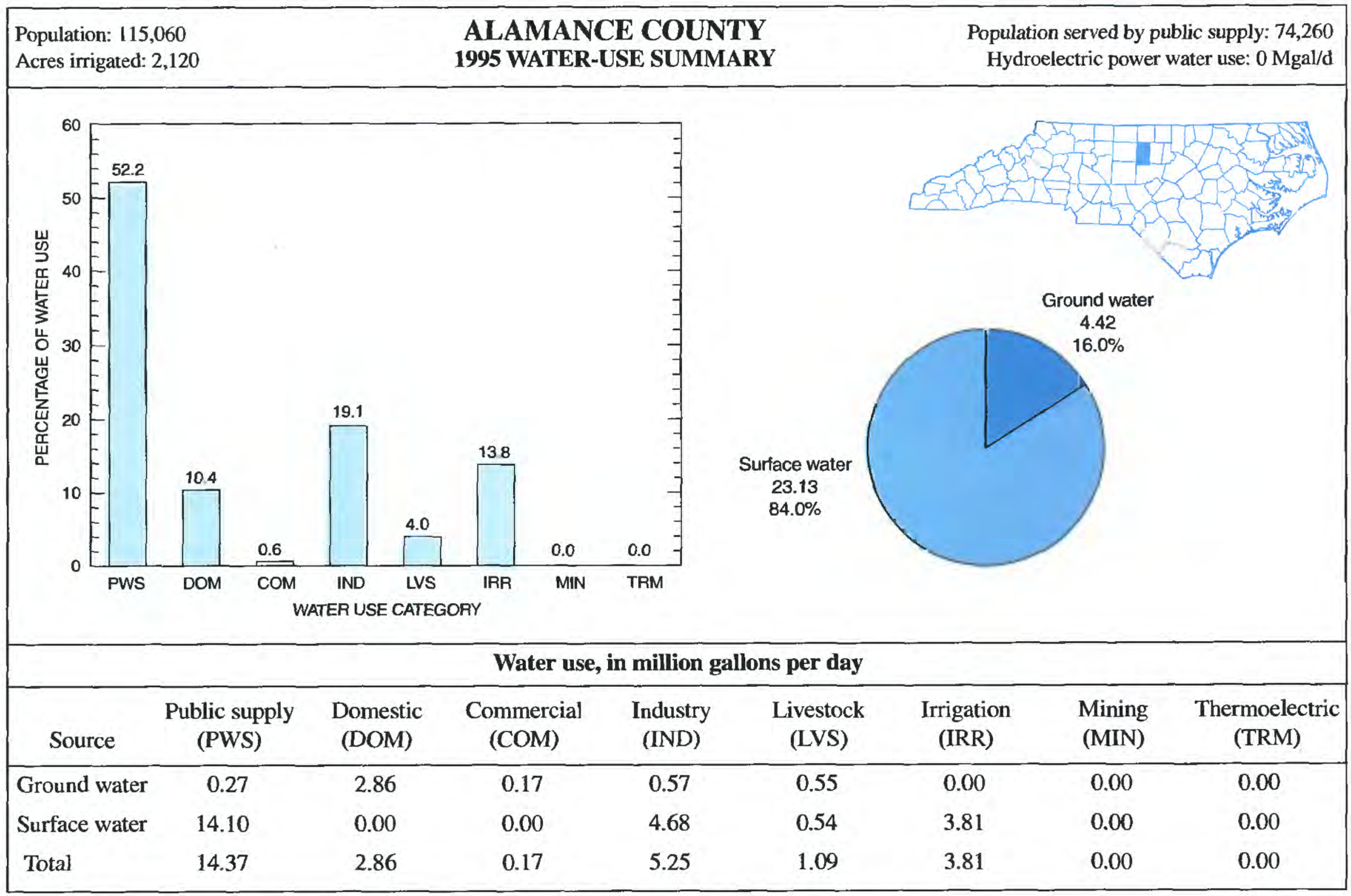

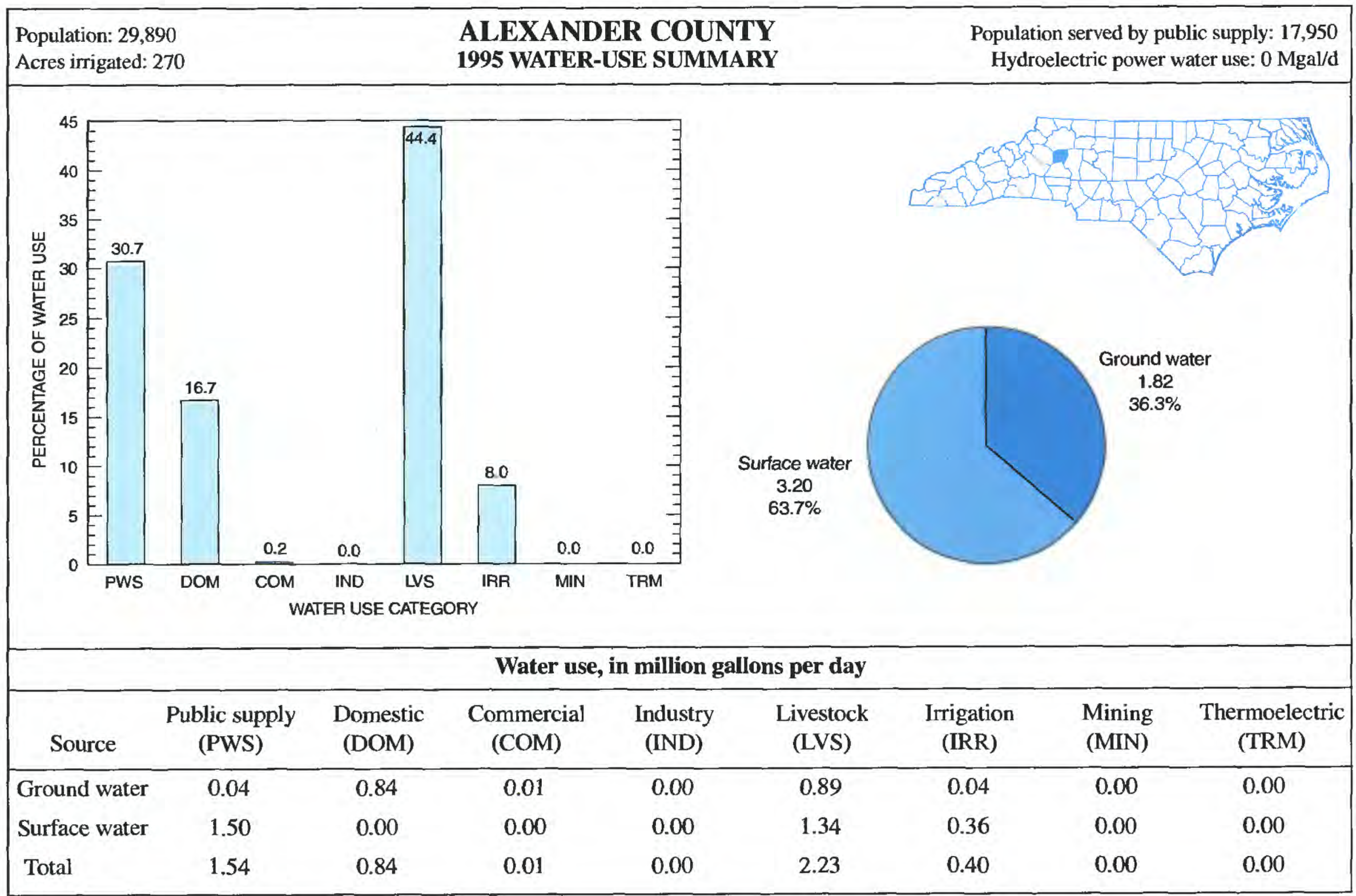




\begin{tabular}{|c|c|c|c|c|c|c|c|c|c|}
\hline \multicolumn{4}{|c|}{$\begin{array}{l}\text { Population: } 9,890 \\
\text { Acres irrigated: } 450\end{array}$} & \multicolumn{3}{|c|}{$\begin{array}{l}\text { ALLEGHANY COUNTY } \\
\text { 1995 WATER-USE SUMMARY }\end{array}$} & \multicolumn{3}{|c|}{$\begin{array}{r}\text { Population served by public supply: } 3,020 \\
\text { Hydroelectric power water use: } 0 \mathrm{Mgal} / \mathrm{d}\end{array}$} \\
\hline 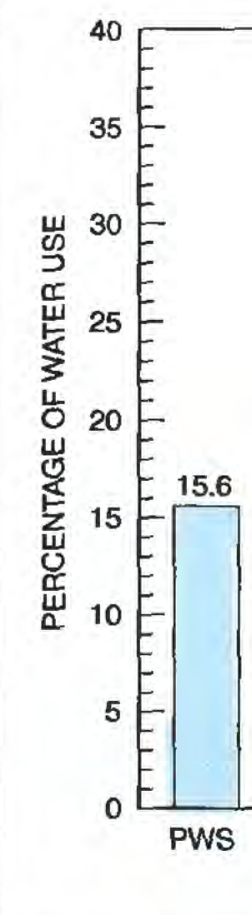 & $\underbrace{}_{\text {DOM }} \Gamma_{\mathrm{COM}}^{1.7}$ & $\frac{\prod_{\text {IND }}^{3.1}}{\prod_{\text {TER USE CA }}}$ & $\frac{\mid}{\text { LVS }}$ & $\frac{|1|}{\text { IRR }}$ & 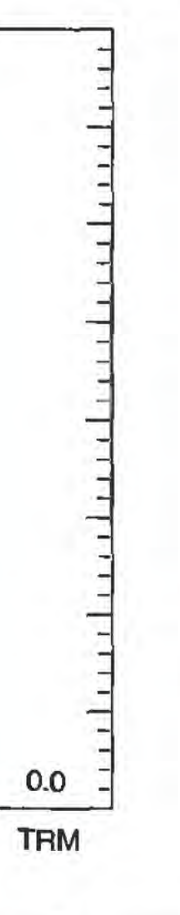 & $\begin{array}{c}\text { Surface water } \\
1.52 \\
52.6 \%\end{array}$ & & & $\begin{array}{l}d \text { water } \\
37 \\
4 \%\end{array}$ \\
\hline \multicolumn{10}{|c|}{ Water use, in million gallons per day } \\
\hline Source & $\begin{array}{l}\text { Public supply } \\
\text { (PWS) }\end{array}$ & $\begin{array}{l}\text { Domestic } \\
\text { (DOM) }\end{array}$ & & $\begin{array}{l}\text { Commercial } \\
\text { (COM) }\end{array}$ & $\begin{array}{l}\text { Industry } \\
\text { (IND) }\end{array}$ & $\begin{array}{l}\text { Livestock } \\
\text { (LVS) }\end{array}$ & $\begin{array}{l}\text { Irrigation } \\
\text { (IRR) }\end{array}$ & $\begin{array}{l}\text { Mining } \\
\text { (MIN) }\end{array}$ & $\begin{array}{l}\text { Thermoelectric } \\
\text { (TRM) }\end{array}$ \\
\hline Ground water & 0.45 & 0.48 & & 0.05 & 0.01 & 0.28 & 0.10 & 0.00 & 0.00 \\
\hline Surface water & 0.00 & 0.00 & & 0.00 & 0.08 & 0.42 & 1.02 & 0.00 & 0.00 \\
\hline Total & 0.45 & 0.48 & & 0.05 & 0.09 & 0.70 & 1.12 & 0.00 & 0.00 \\
\hline
\end{tabular}

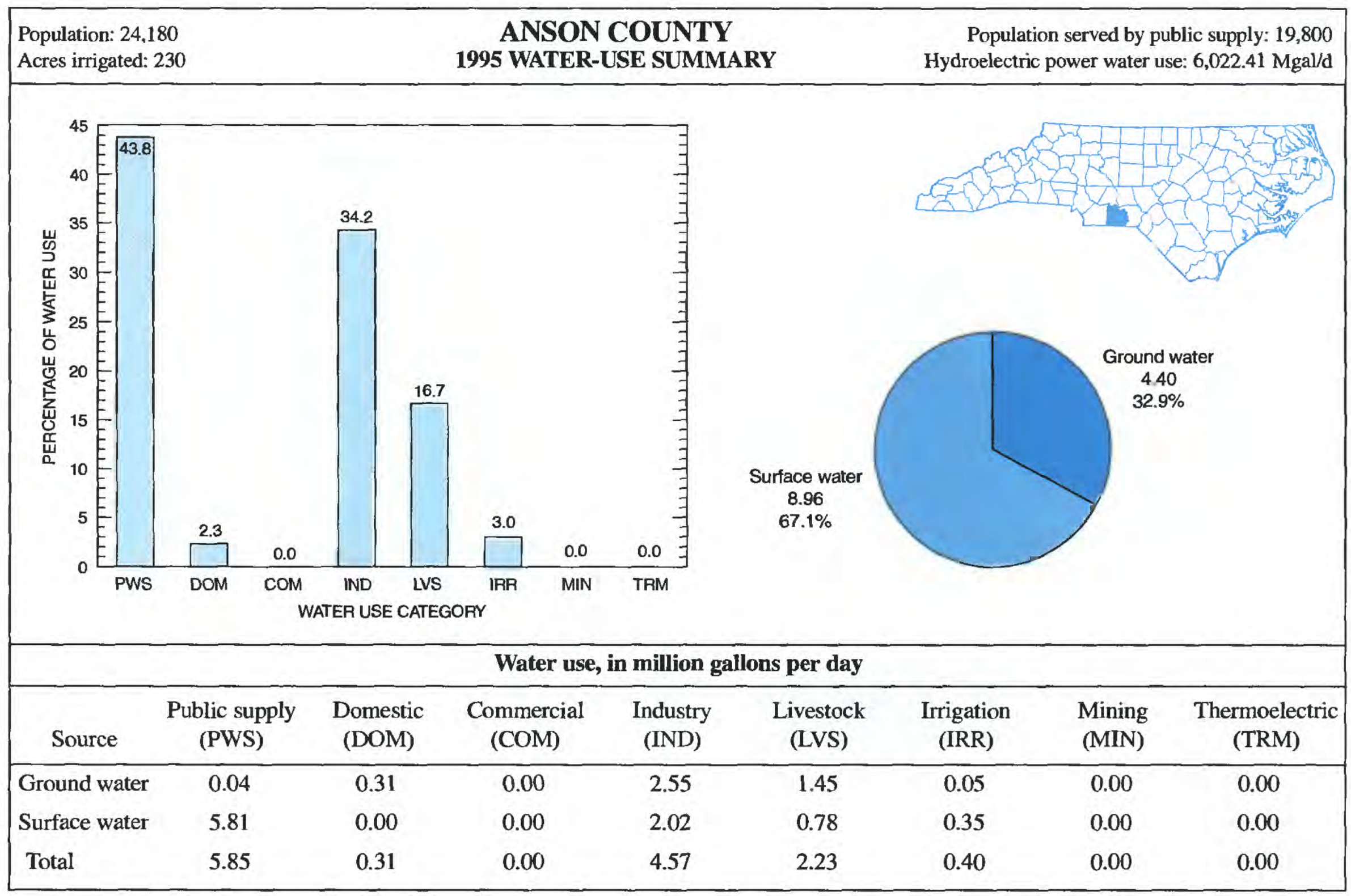




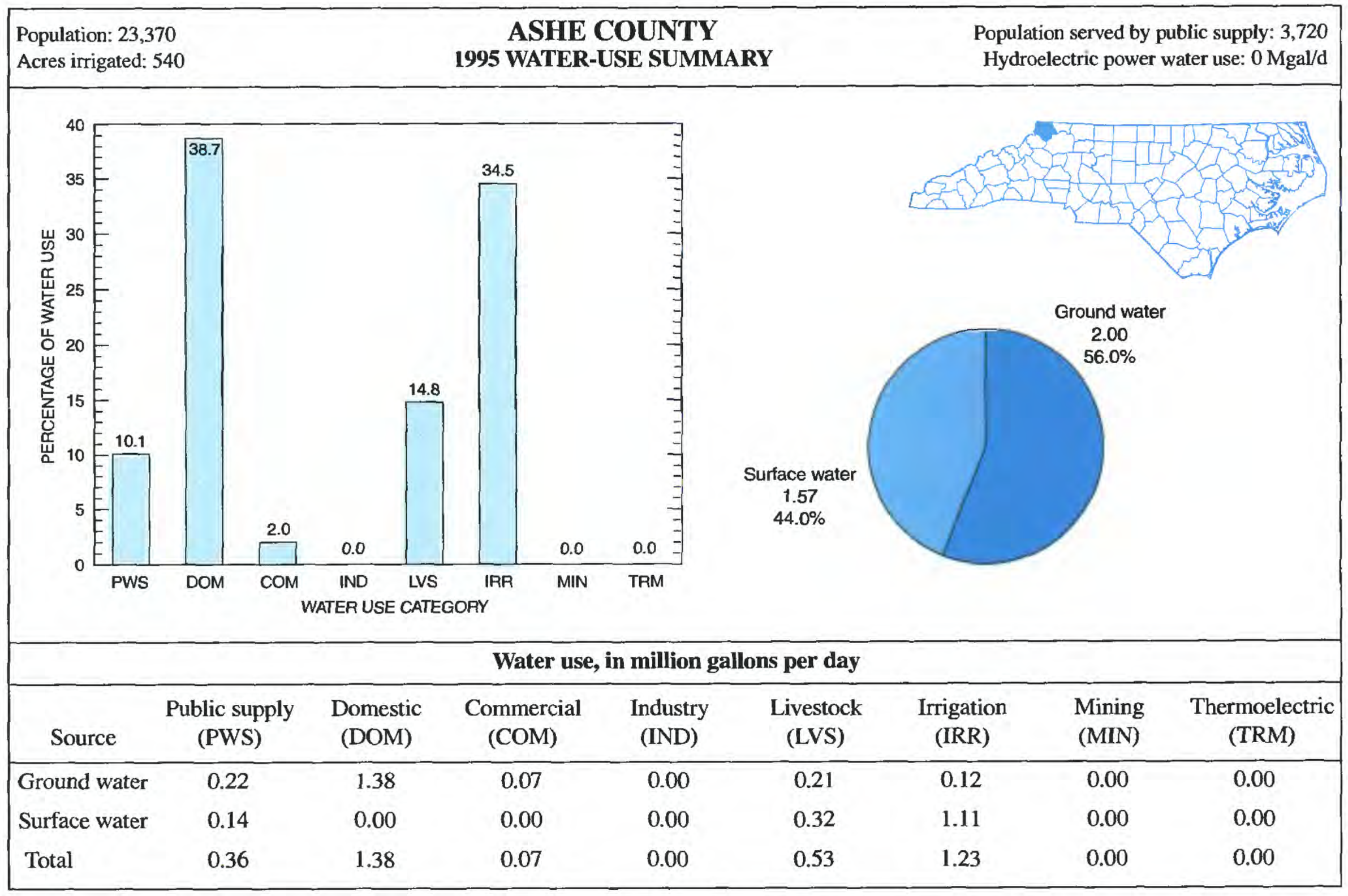

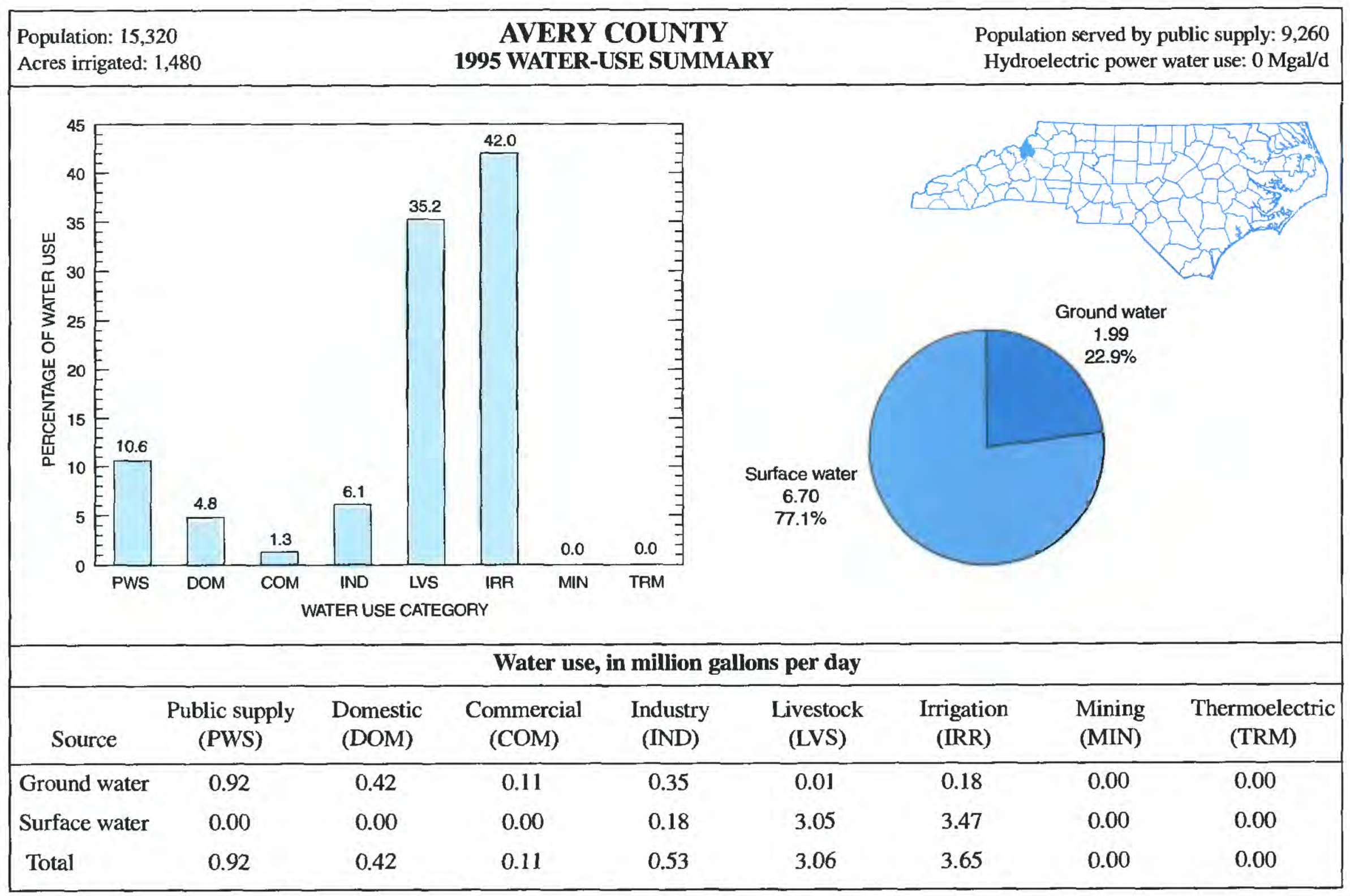




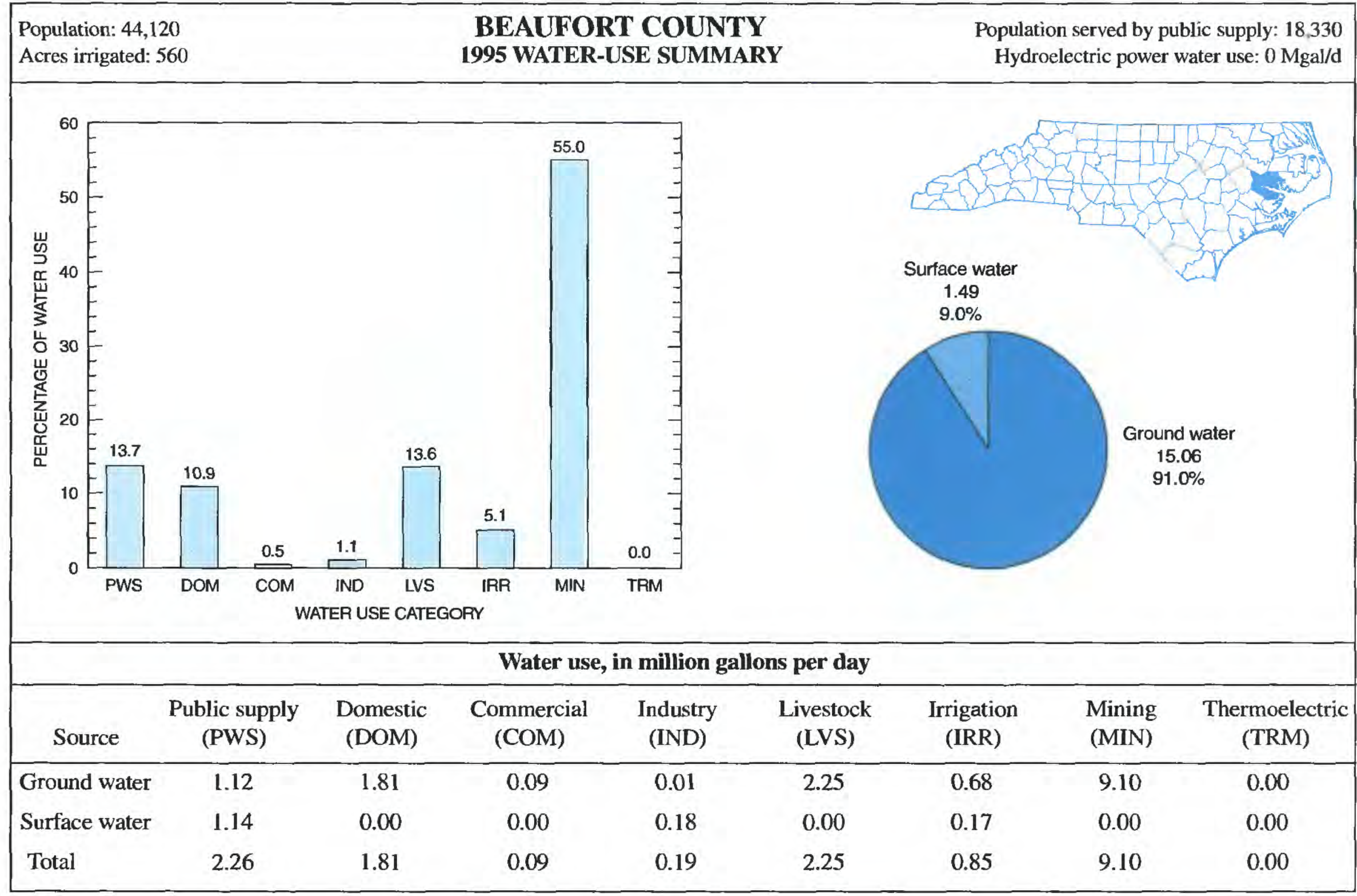

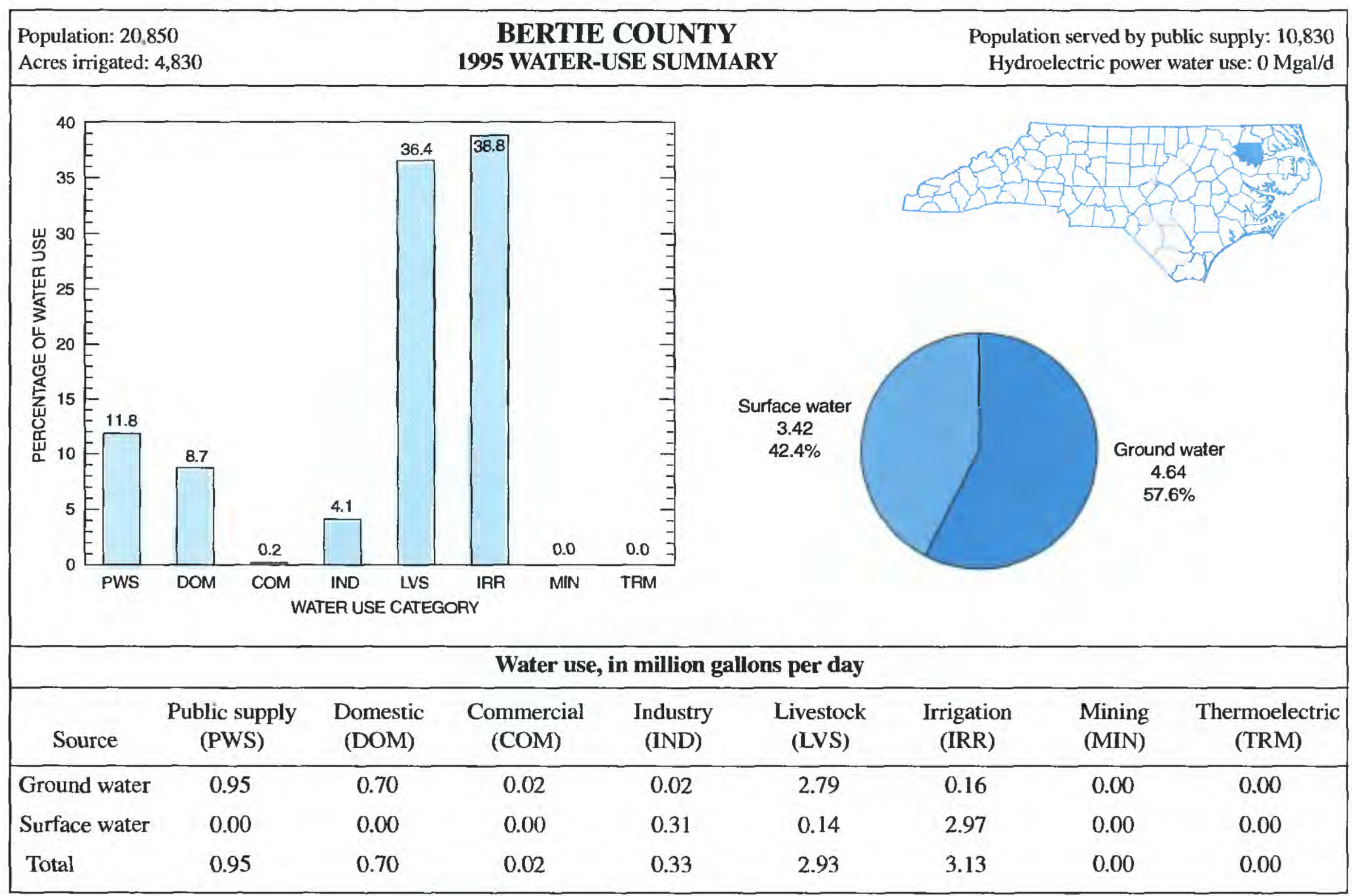




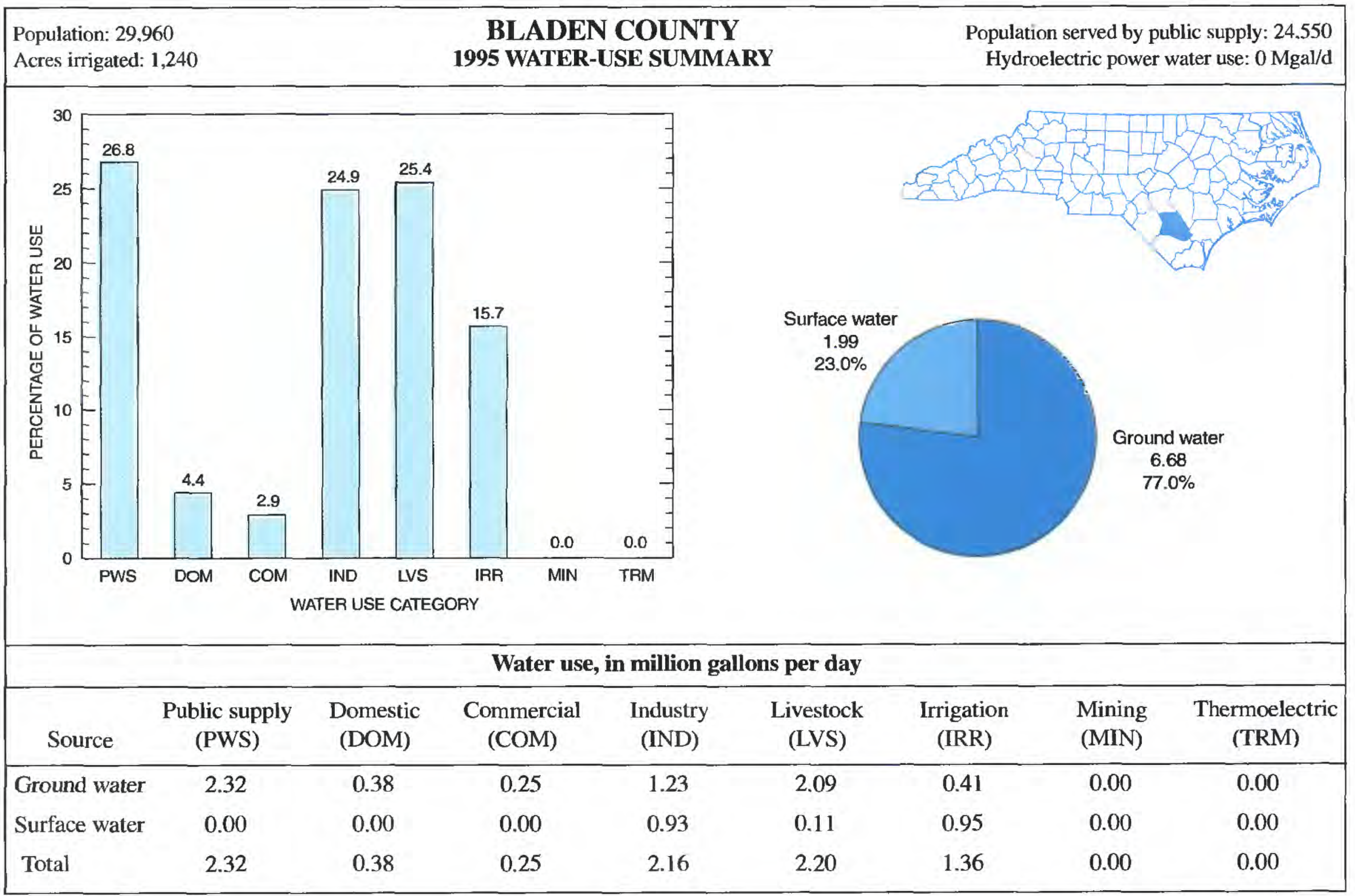

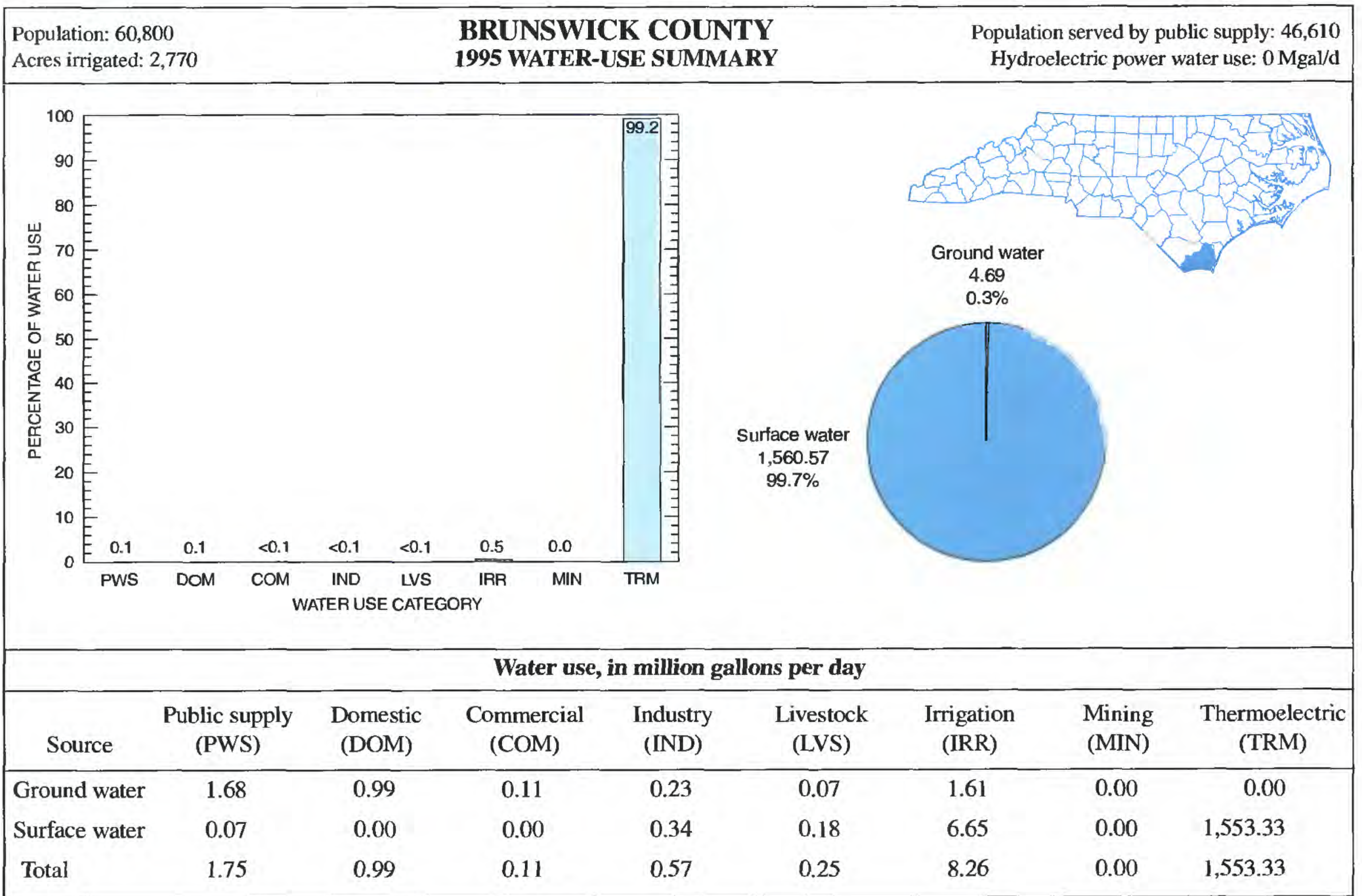




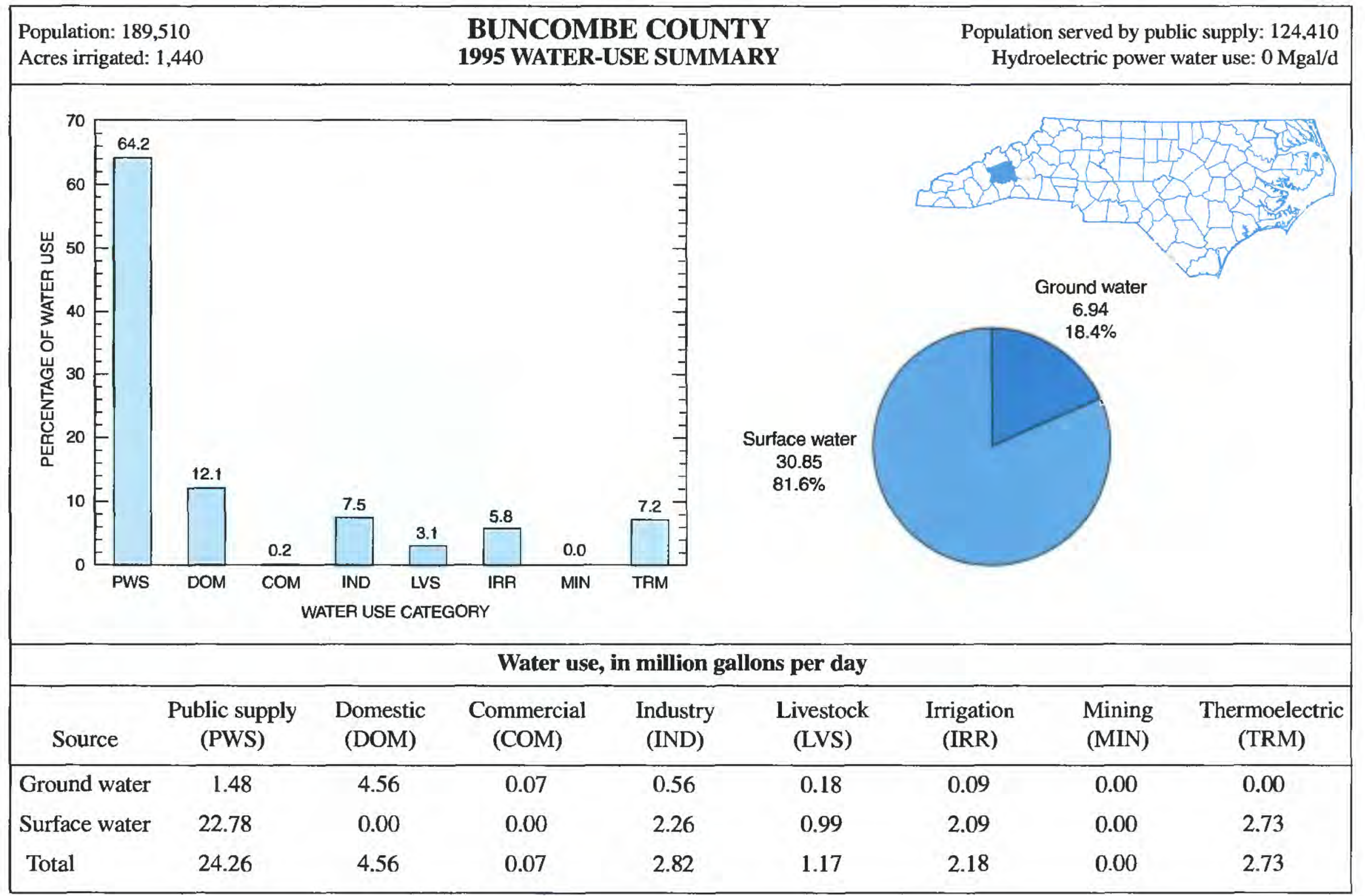

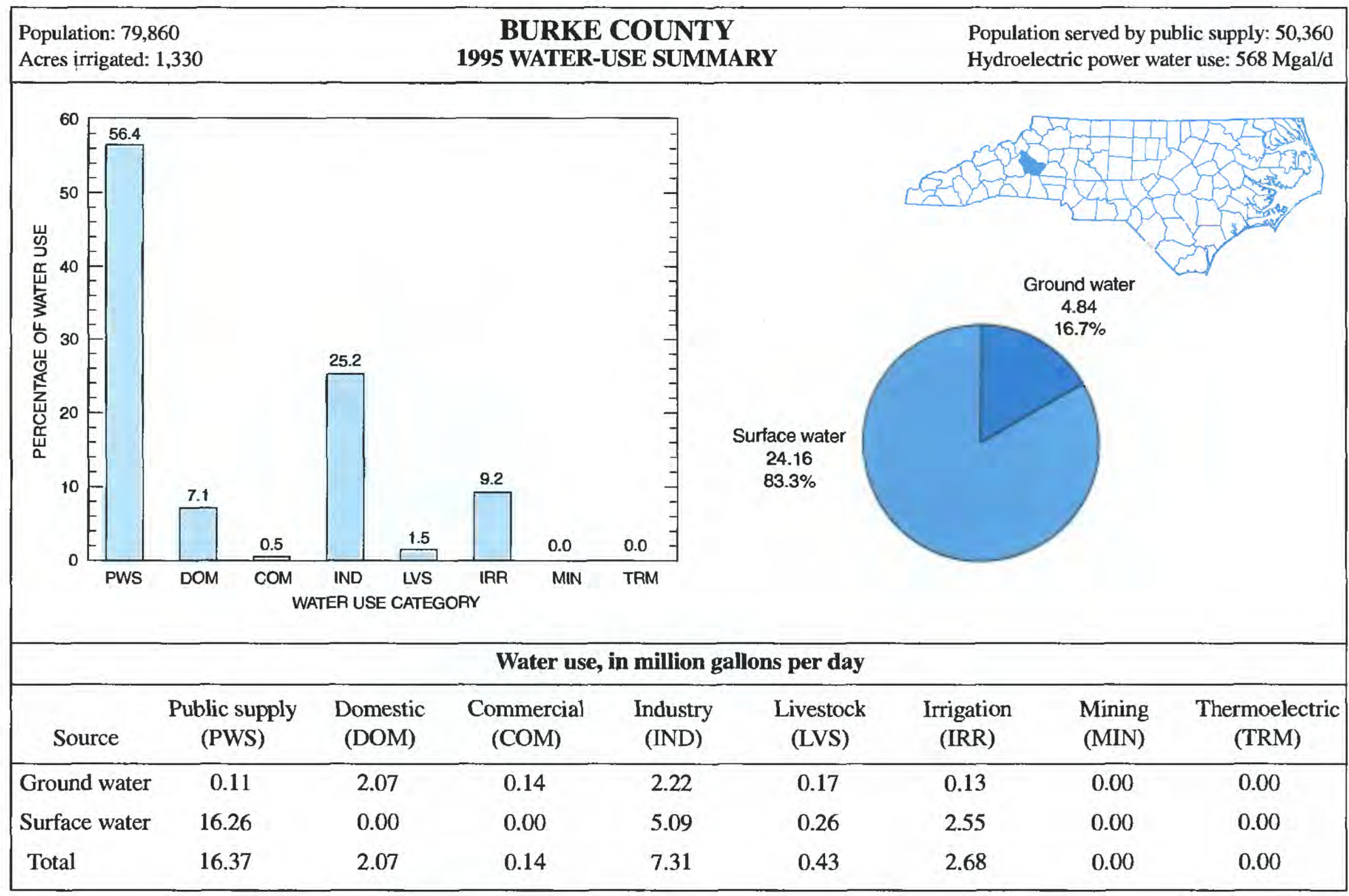




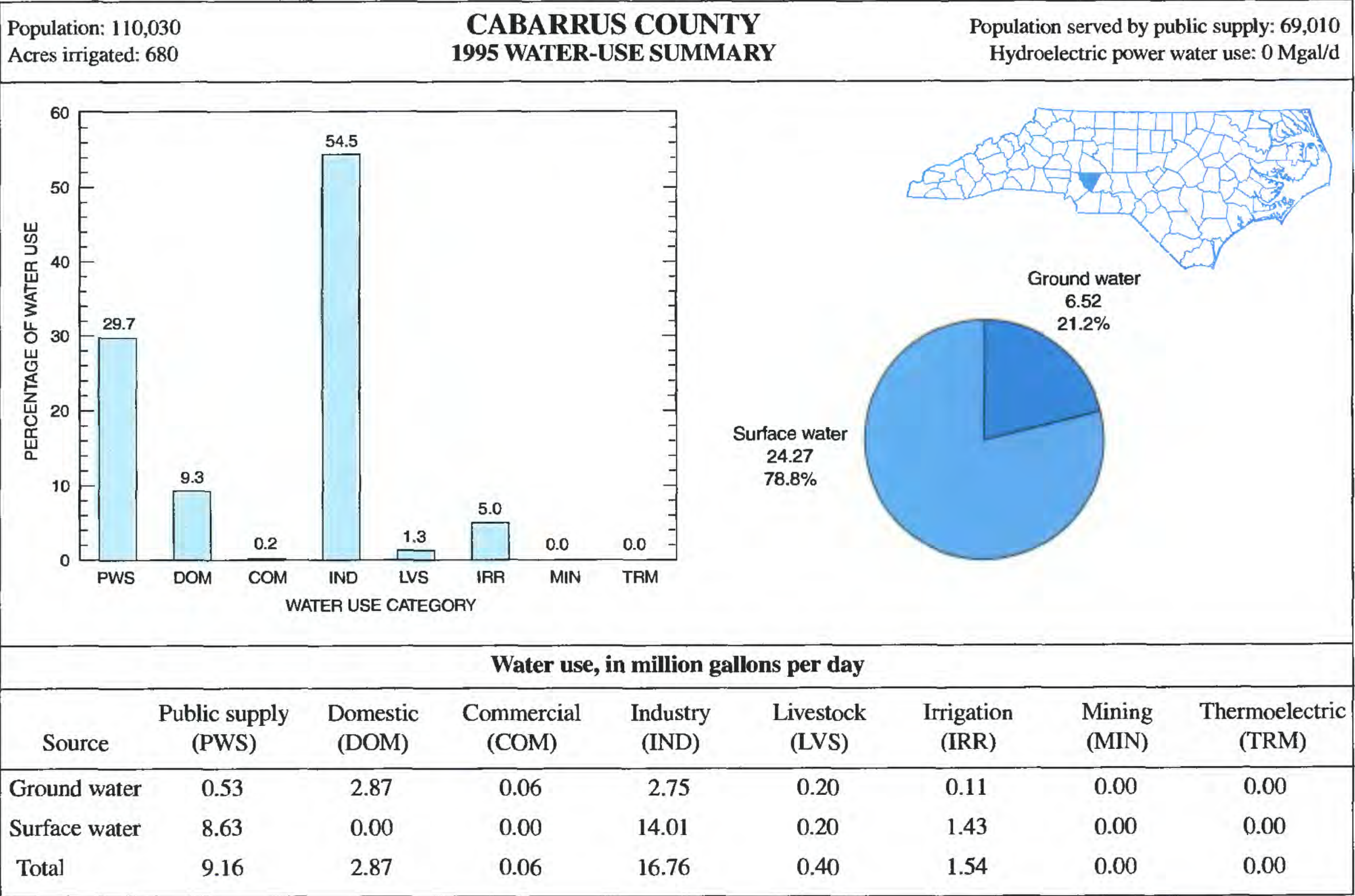

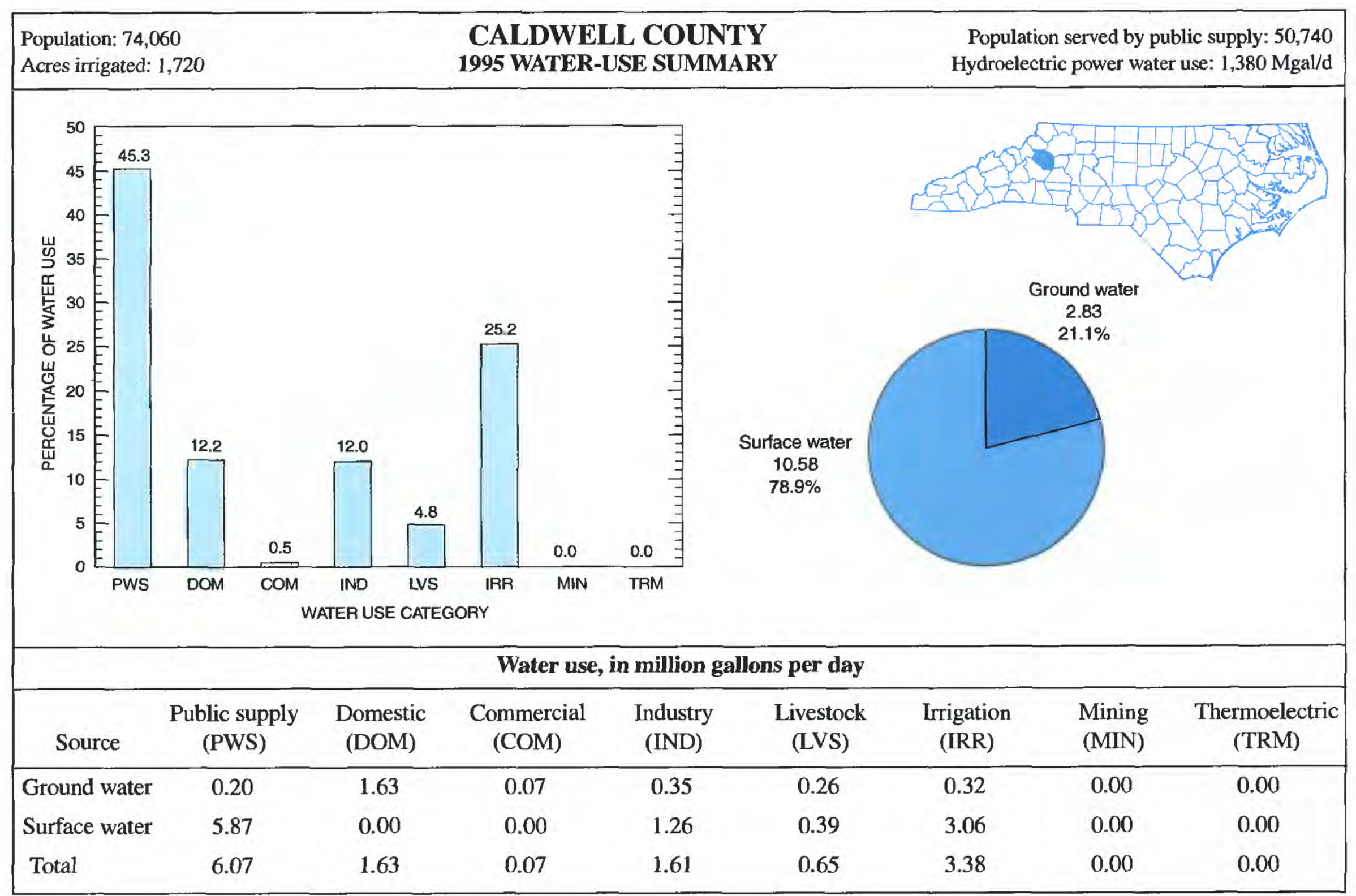




\begin{tabular}{|c|c|c|c|c|c|c|c|c|}
\hline \multicolumn{2}{|c|}{$\begin{array}{l}\text { Population: } 6,450 \\
\text { Acres irrigated: } 70\end{array}$} & \multicolumn{4}{|c|}{$\begin{array}{c}\text { CAMDEN COUNTY } \\
\text { 1995 WATER-USE SUMMARY }\end{array}$} & \multicolumn{3}{|c|}{$\begin{array}{r}\text { Population served by public supply: } 5,510 \\
\text { Hydroelectric power water use: } 0 \text { Mgal/d }\end{array}$} \\
\hline \multicolumn{9}{|l|}{ 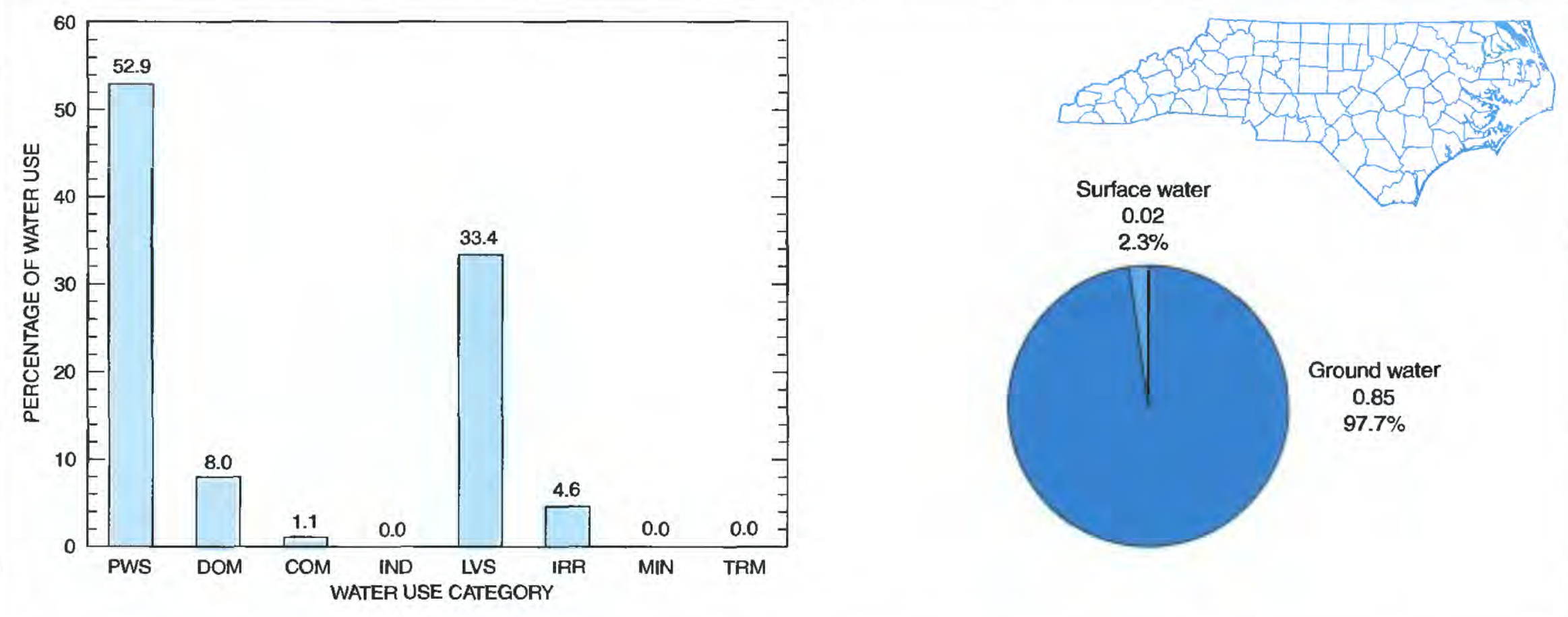 } \\
\hline \multicolumn{9}{|c|}{ Water use, in million gallons per day } \\
\hline Source & $\begin{array}{l}\text { Public supply } \\
\text { (PWS) }\end{array}$ & $\begin{array}{l}\text { Domestic } \\
\text { (DOM) }\end{array}$ & $\begin{array}{c}\text { Commercial } \\
\text { (COM) }\end{array}$ & $\begin{array}{l}\text { Industry } \\
\text { (IND) }\end{array}$ & $\begin{array}{l}\text { Livestock } \\
\text { (LVS) }\end{array}$ & $\begin{array}{l}\text { Irrigation } \\
\text { (IRR) }\end{array}$ & $\begin{array}{l}\text { Mining } \\
\text { (MIN) }\end{array}$ & $\begin{array}{l}\text { Thermoelectric } \\
\text { (TRM) }\end{array}$ \\
\hline Ground water & 0.46 & 0.07 & 0.01 & 0.00 & 0.29 & 0.02 & 0.00 & 0.00 \\
\hline Surface water & 0.00 & 0.00 & 0.00 & 0.00 & 0.00 & 0.02 & 0.00 & 0.00 \\
\hline Total & 0.46 & 0.07 & 0.01 & 0.00 & 0.29 & 0.04 & 0.00 & 0.00 \\
\hline
\end{tabular}

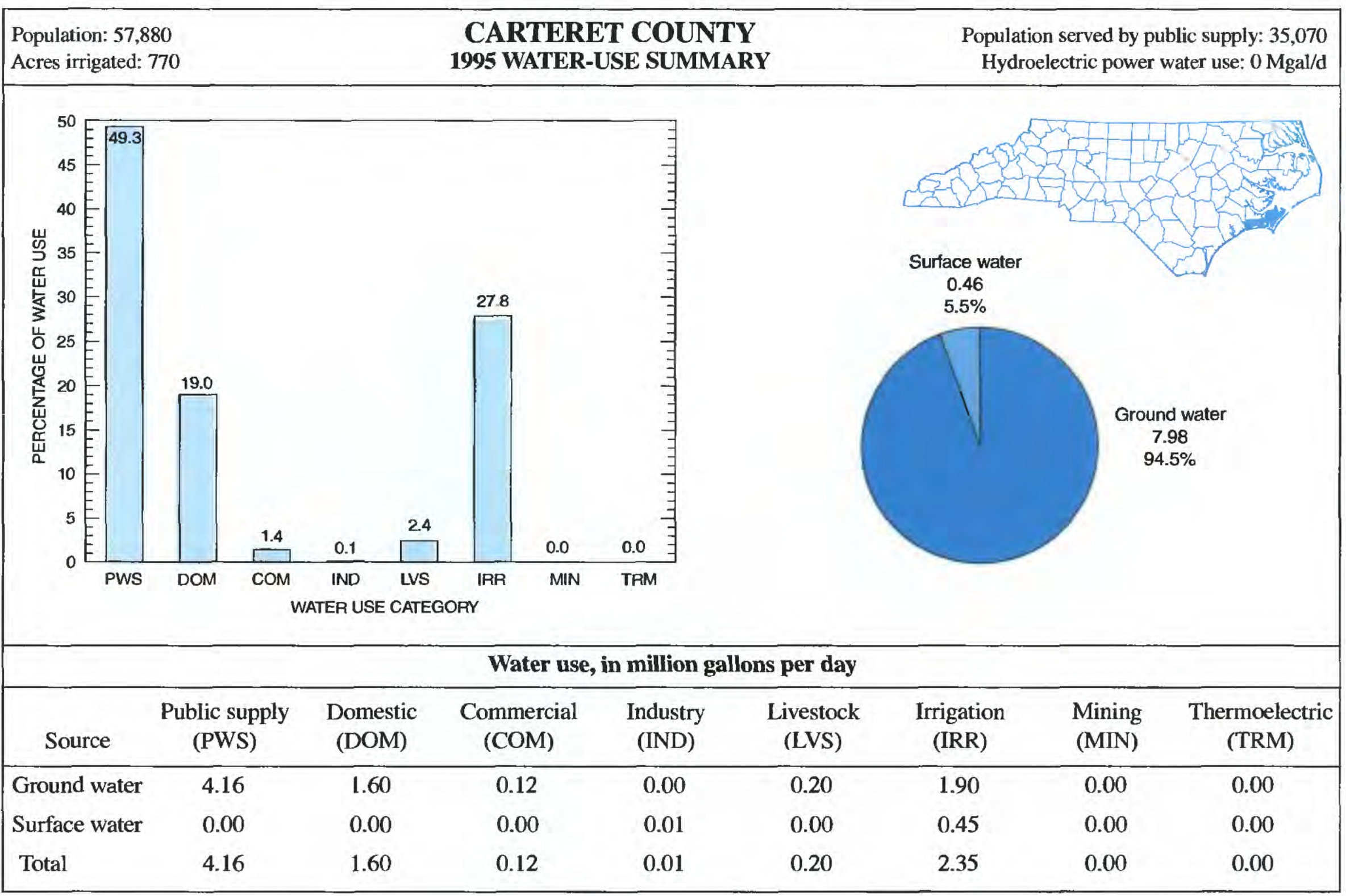




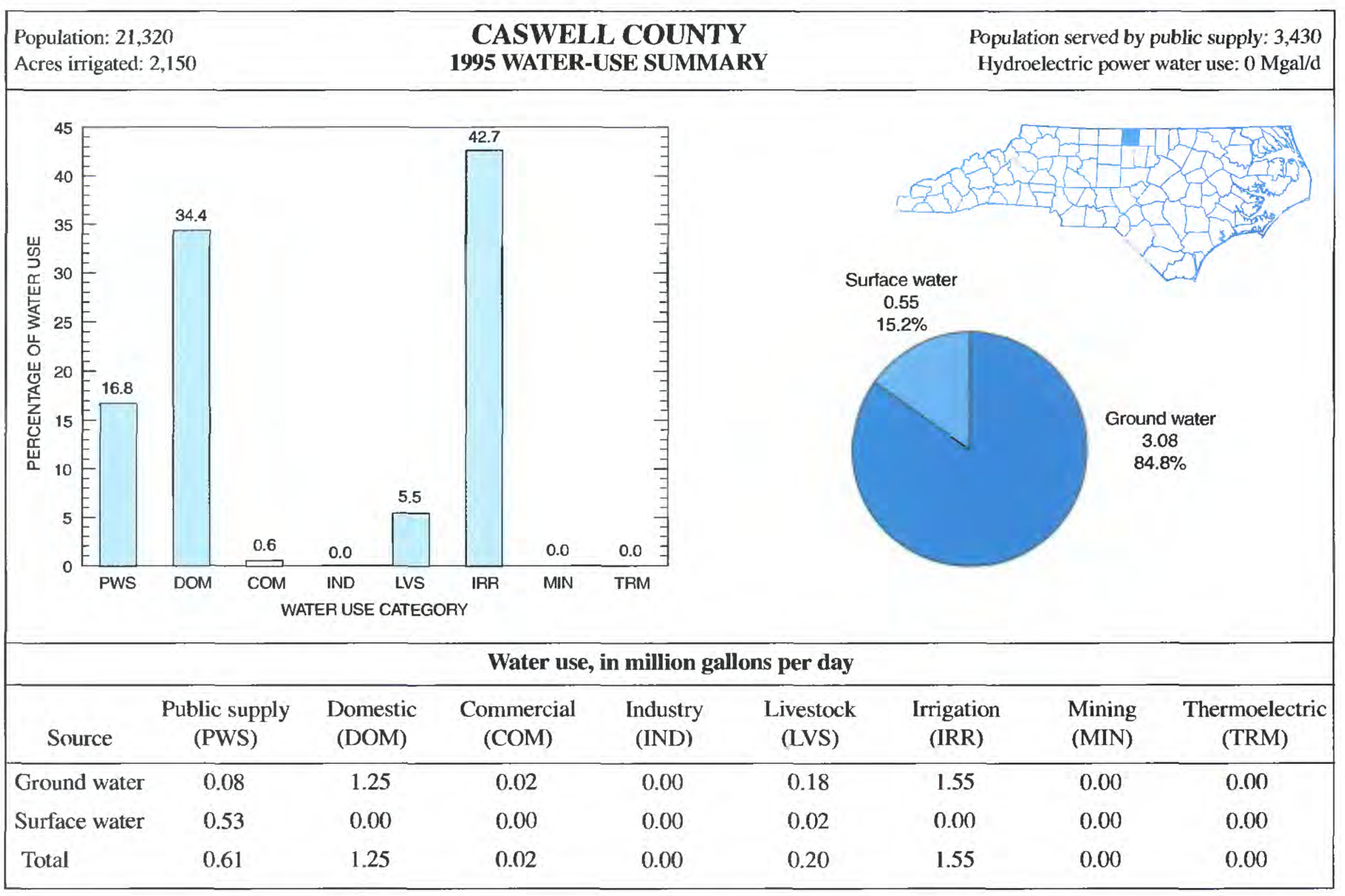

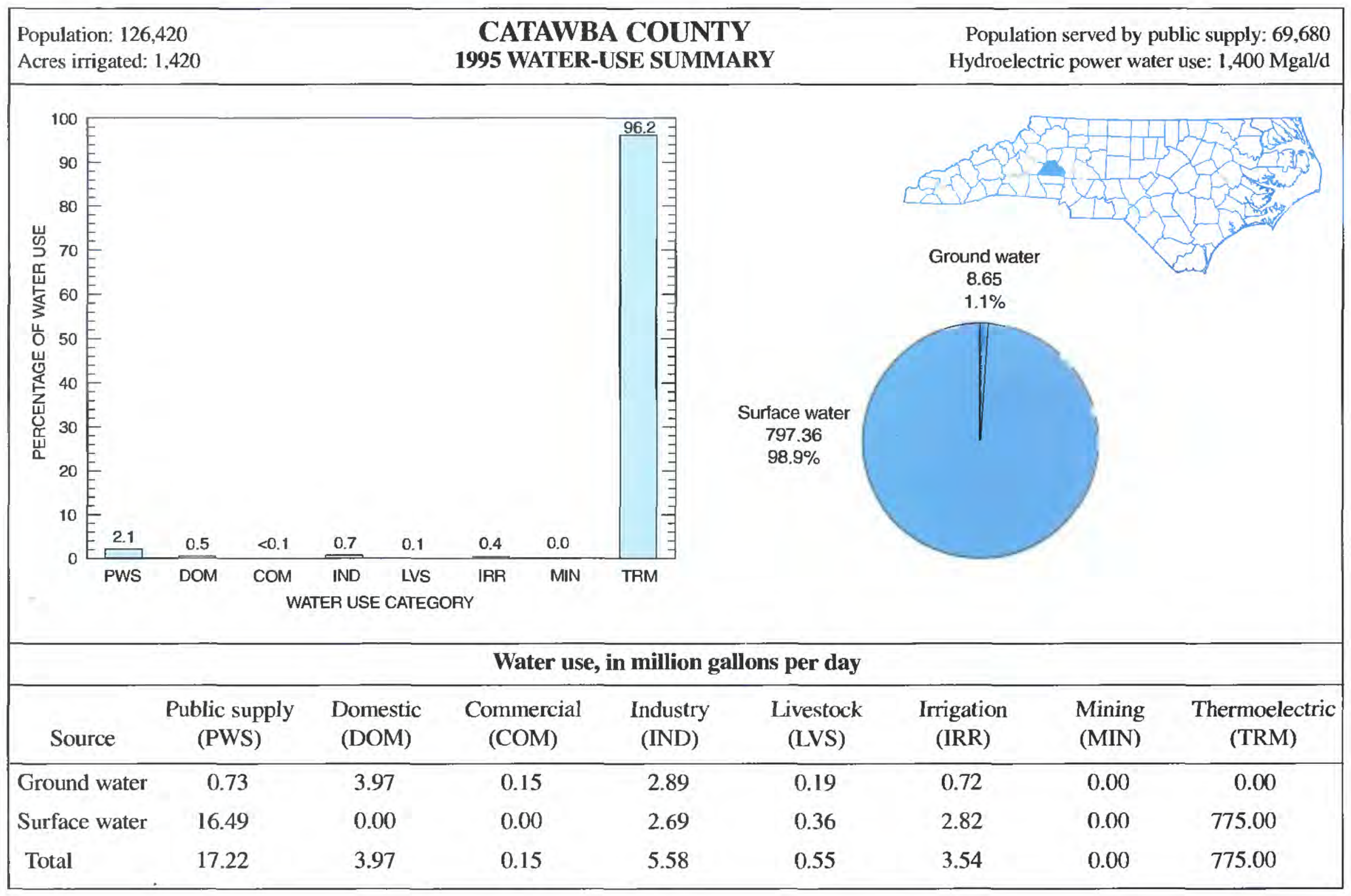




\begin{tabular}{|c|c|c|c|c|c|c|c|c|c|c|c|}
\hline \multicolumn{3}{|c|}{$\begin{array}{l}\text { Population: } 42,540 \\
\text { Acres irrigated: } 630\end{array}$} & \multicolumn{6}{|c|}{$\begin{array}{l}\text { CHATHAM COUNTY } \\
\text { 1995 WATER-USE SUMMARY }\end{array}$} & \multicolumn{3}{|c|}{$\begin{array}{l}\text { Population served by public supply: } 17,580 \\
\text { Hydroelectric power water use: } 0 \mathrm{Mgal} / \mathrm{d}\end{array}$} \\
\hline 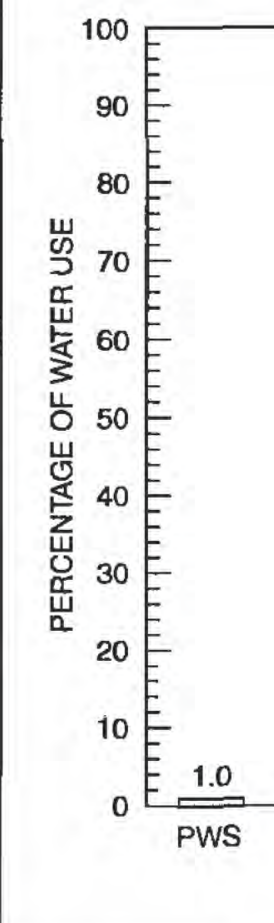 & 0.4 & $\begin{array}{l}<0.1 \\
\mathrm{COM}\end{array}$ & $\begin{array}{r}0.1 \\
\text { IND } \\
\text { HER U: }\end{array}$ & $\begin{array}{l}\frac{1.3}{\text { LVS }} \\
\text { CATE }\end{array}$ & $\begin{array}{c}0.1 \\
\text { IRR } \\
\text { ORY }\end{array}$ & $\frac{0.0}{\mathrm{MIN}}$ & 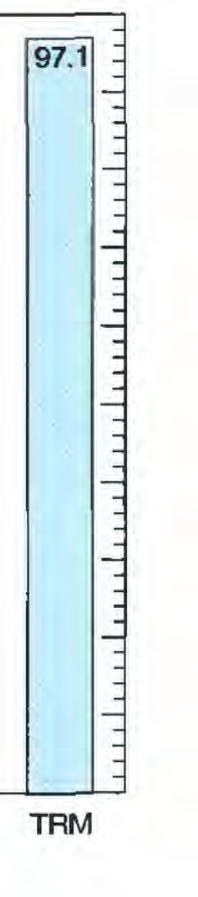 & \multirow[t]{2}{*}{$\begin{array}{c}\text { Surface water } \\
390.35 \\
98.5 \% \\
\\
\\
\\
\text { gallons per day }\end{array}$} & $\begin{array}{c}\text { Ground w } \\
6.14 \\
1.5 \%\end{array}$ & & \\
\hline \multicolumn{11}{|c|}{ Water use, in million gallons per day } & \\
\hline Source & \multicolumn{2}{|c|}{$\begin{array}{l}\text { Public supply } \\
\quad \text { (PWS) }\end{array}$} & \multicolumn{2}{|c|}{$\begin{array}{l}\text { Domestic } \\
\text { (DOM) }\end{array}$} & \multicolumn{2}{|c|}{$\begin{array}{l}\text { Commercial } \\
(\mathrm{COM})\end{array}$} & $\begin{array}{l}\text { Industry } \\
\text { (IND) }\end{array}$ & $\begin{array}{l}\text { Livestock } \\
\text { (LVS) }\end{array}$ & $\begin{array}{l}\text { Irrigation } \\
\text { (IRR) }\end{array}$ & $\begin{array}{l}\text { Mining } \\
(\mathrm{MIN})\end{array}$ & $\begin{array}{l}\text { Thermoelectric } \\
\text { (TRM) }\end{array}$ \\
\hline Ground water & \multicolumn{2}{|c|}{0.49} & \multicolumn{2}{|c|}{1.75} & \multicolumn{2}{|c|}{0.03} & 0.06 & 3.76 & 0.05 & 0.00 & 0.00 \\
\hline Surface water & \multicolumn{2}{|c|}{3.31} & 0. & & \multicolumn{2}{|c|}{0.04} & 0.36 & 1.25 & 0.39 & 0.00 & 385.00 \\
\hline Total & \multicolumn{2}{|c|}{3.80} & 1. & & \multicolumn{2}{|c|}{0.07} & 0.42 & 5.01 & 0.44 & 0.00 & 385.00 \\
\hline
\end{tabular}

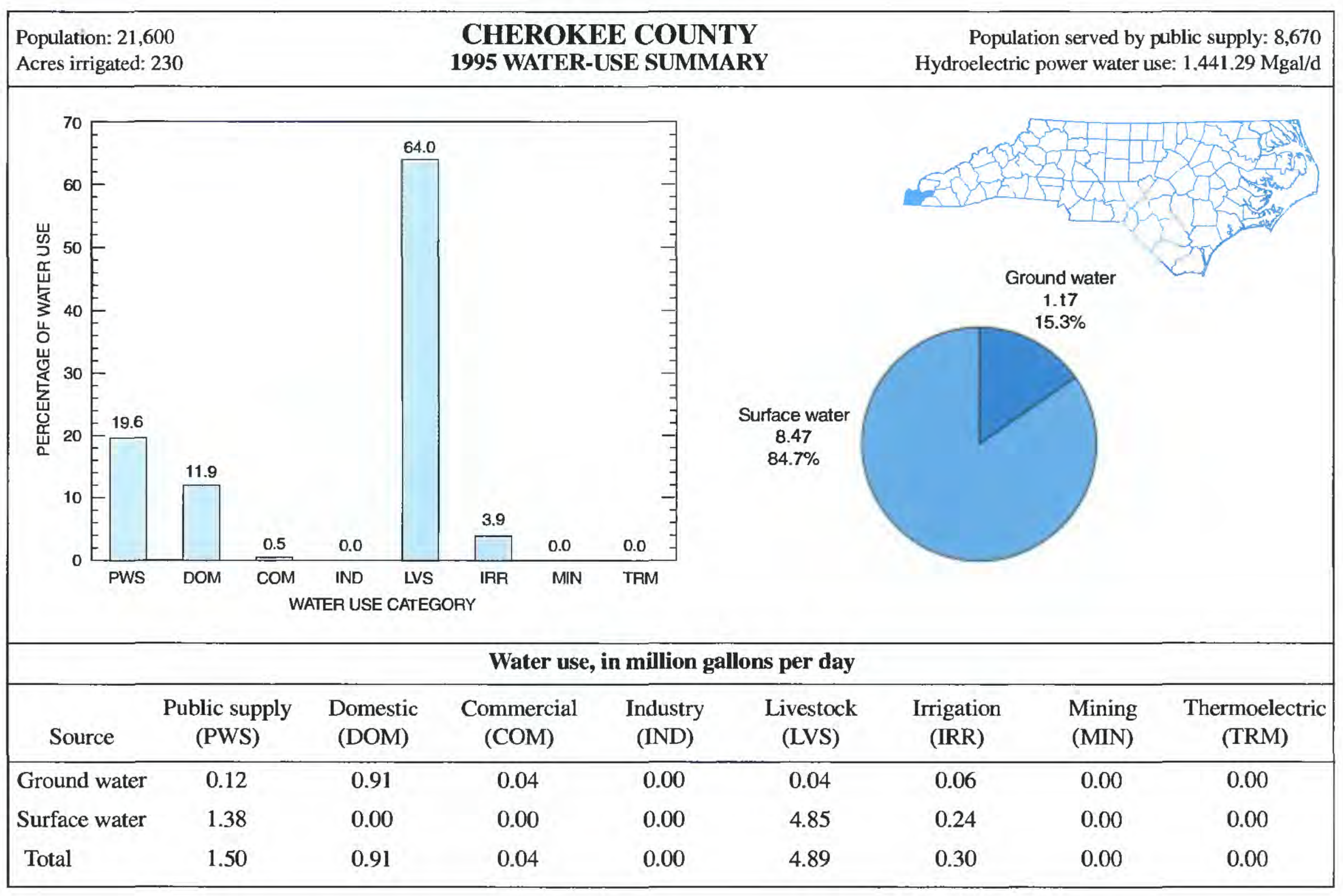




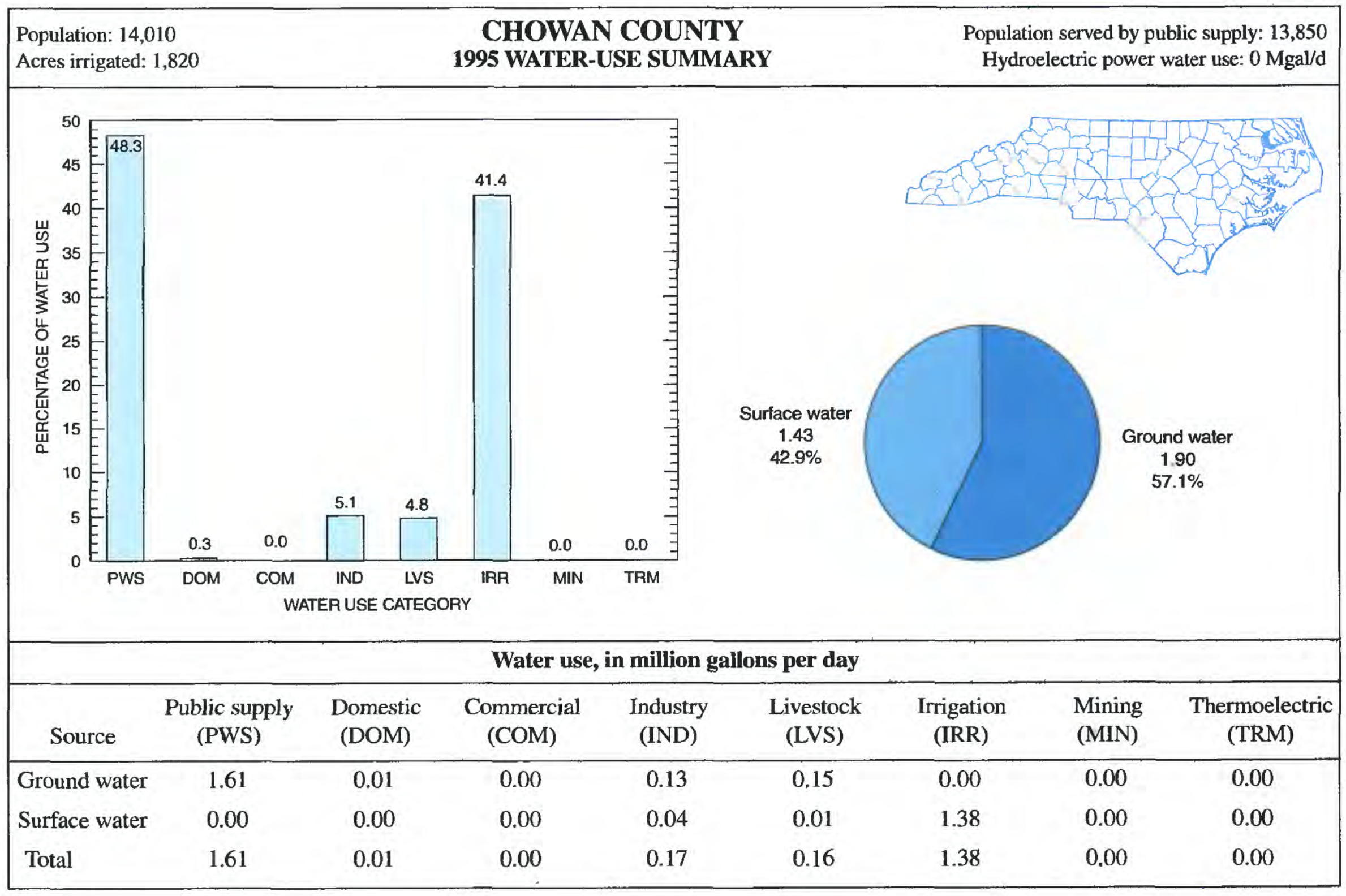

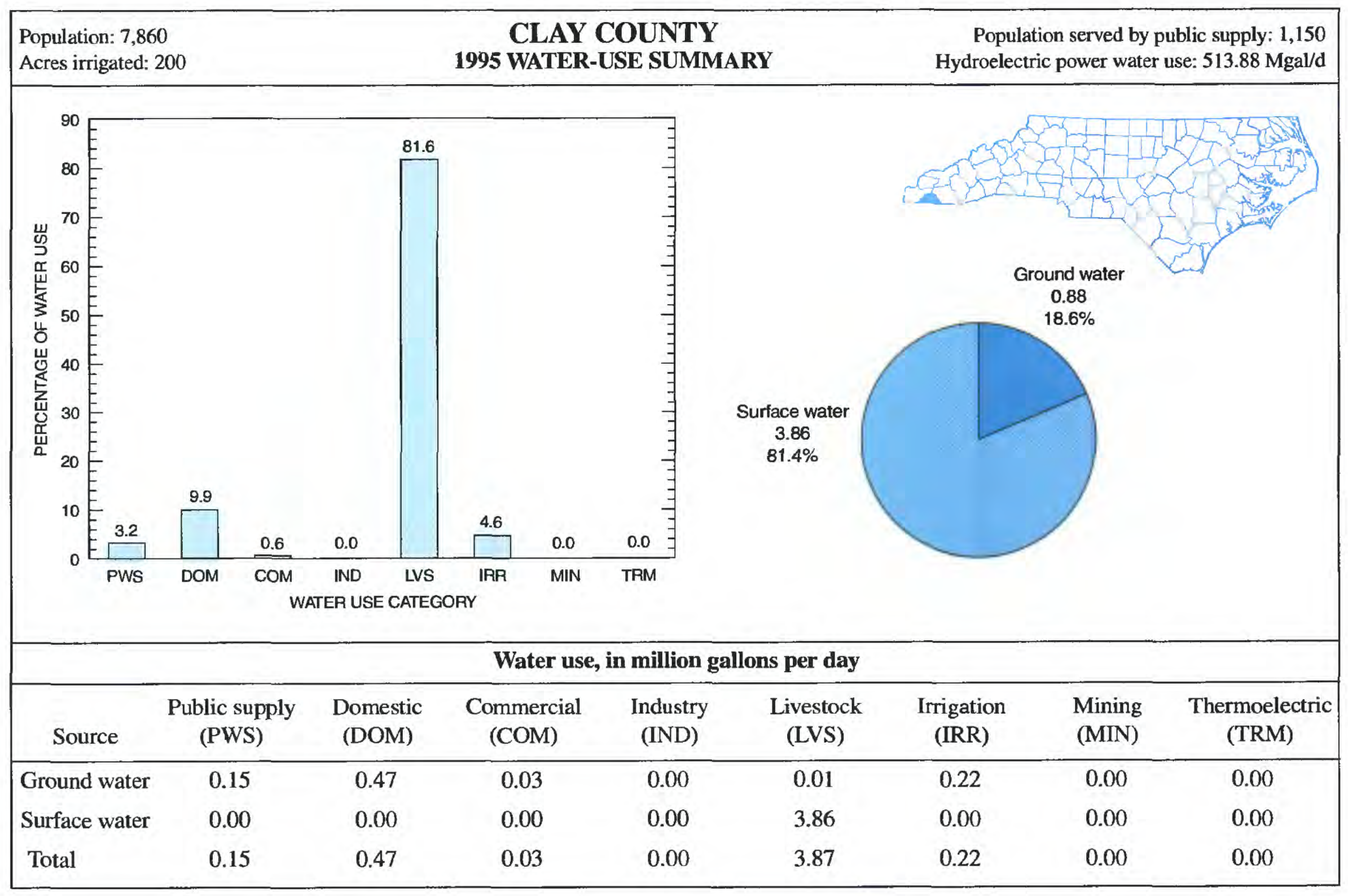




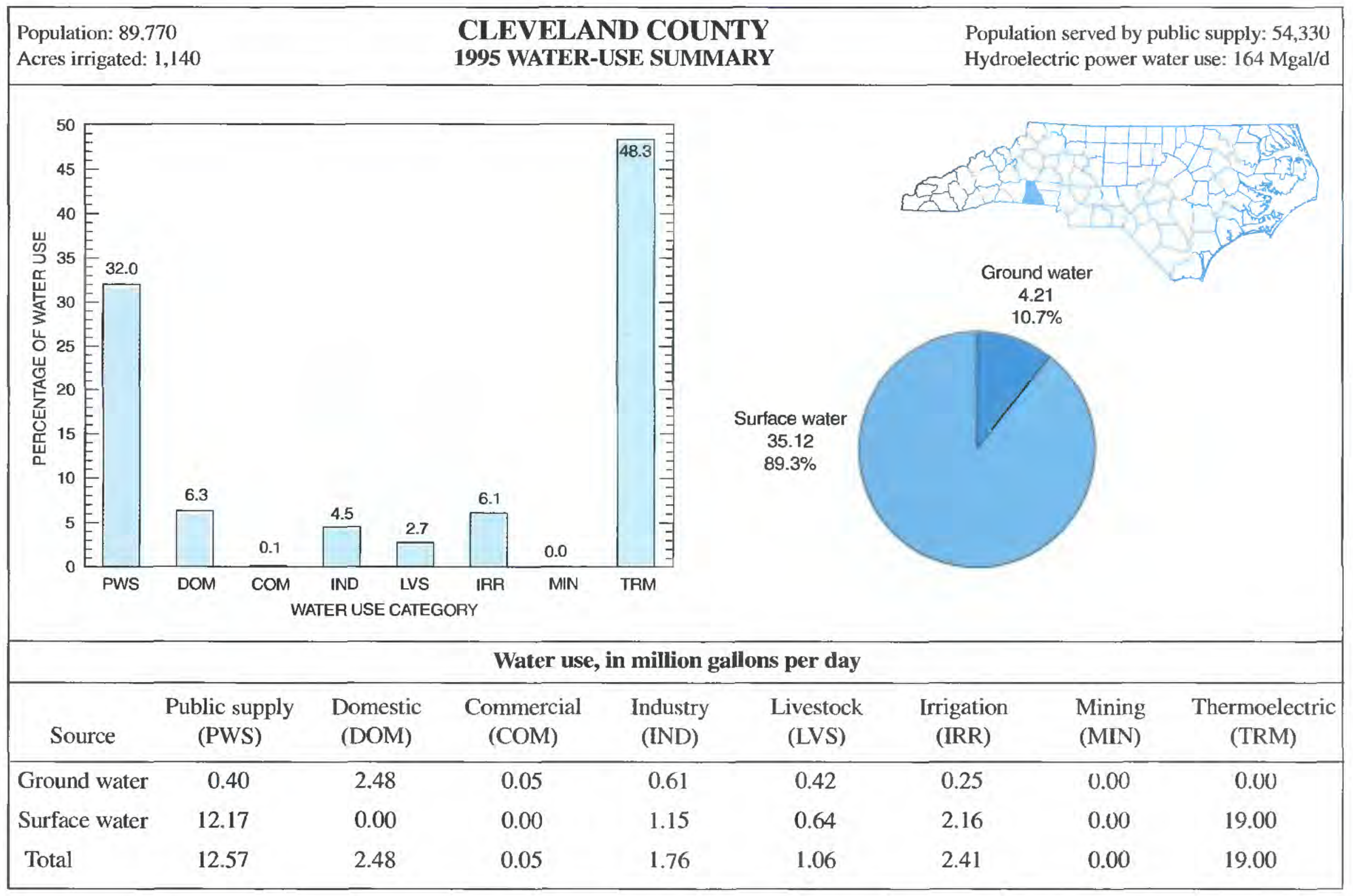

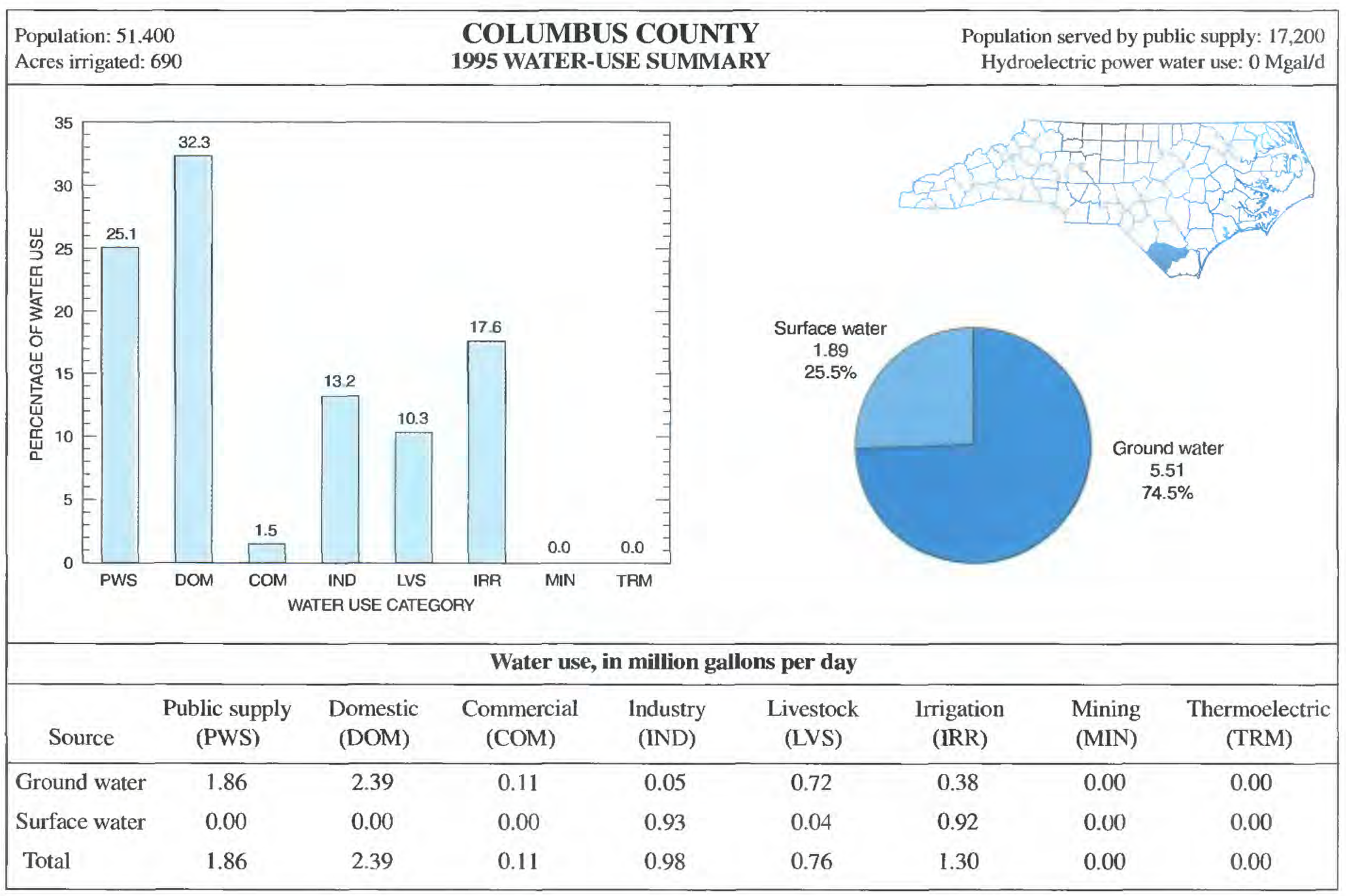




\begin{tabular}{|c|c|c|c|c|c|c|c|c|c|}
\hline \multicolumn{3}{|c|}{$\begin{array}{l}\text { Population: } 85,520 \\
\text { Acres irrigated: } 1,240\end{array}$} & \multicolumn{4}{|c|}{$\begin{array}{c}\text { CRAVEN COUNTY } \\
\text { 1995 WATER-USE SUMMARY }\end{array}$} & \multicolumn{3}{|c|}{$\begin{array}{l}\text { Population served by public supply: } 81.190 \\
\text { Hydroelectric power water use: } 0 \mathrm{Mgal} / \mathrm{d}\end{array}$} \\
\hline \multicolumn{10}{|l|}{ 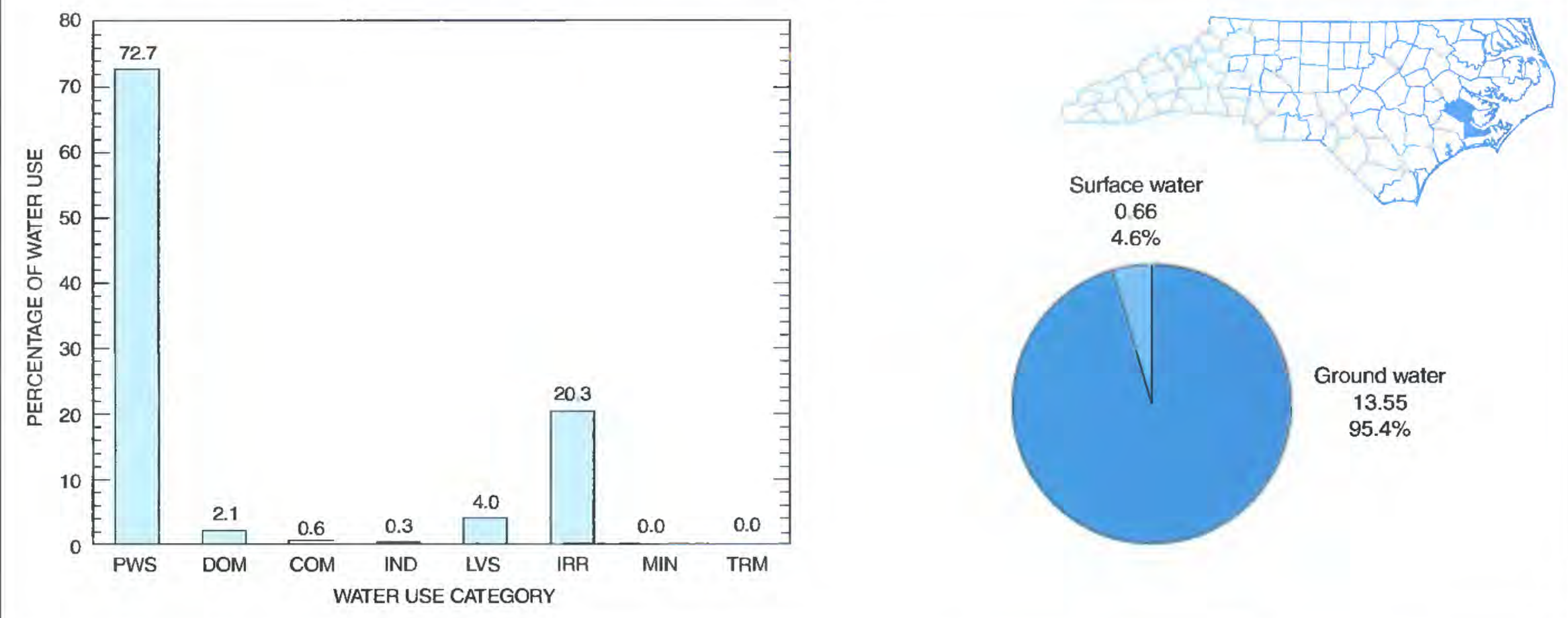 } \\
\hline \multicolumn{10}{|c|}{ Water use, in million gallons per day } \\
\hline Source & $\begin{array}{r}\text { Public } \\
\quad \text { (PV }\end{array}$ & $\begin{array}{l}\text { upply } \\
\text { S) }\end{array}$ & $\begin{array}{l}\text { Domestic } \\
\text { (DOM) }\end{array}$ & $\begin{array}{l}\text { Commercial } \\
\text { (COM) }\end{array}$ & $\begin{array}{l}\text { Industry } \\
\text { (IND) }\end{array}$ & $\begin{array}{l}\text { Livestock } \\
\text { (LVS) }\end{array}$ & $\begin{array}{l}\text { Irrigation } \\
\text { (IRR) }\end{array}$ & $\begin{array}{l}\text { Mining } \\
(\mathrm{MIN})\end{array}$ & $\begin{array}{l}\text { Thermoelectric } \\
\text { (TRM) }\end{array}$ \\
\hline Ground water & 10.3 & & 0.30 & 0.08 & 0.00 & 0.53 & 2.31 & 0.00 & 0.00 \\
\hline Surface water & 0.8 & & 0.00 & 0.00 & 0.04 & 0.04 & 0.58 & 0.00 & 0.00 \\
\hline Total & 10.3 & & 0.30 & 0.08 & 0.04 & 0.57 & 2.89 & 0.00 & 0.00 \\
\hline
\end{tabular}

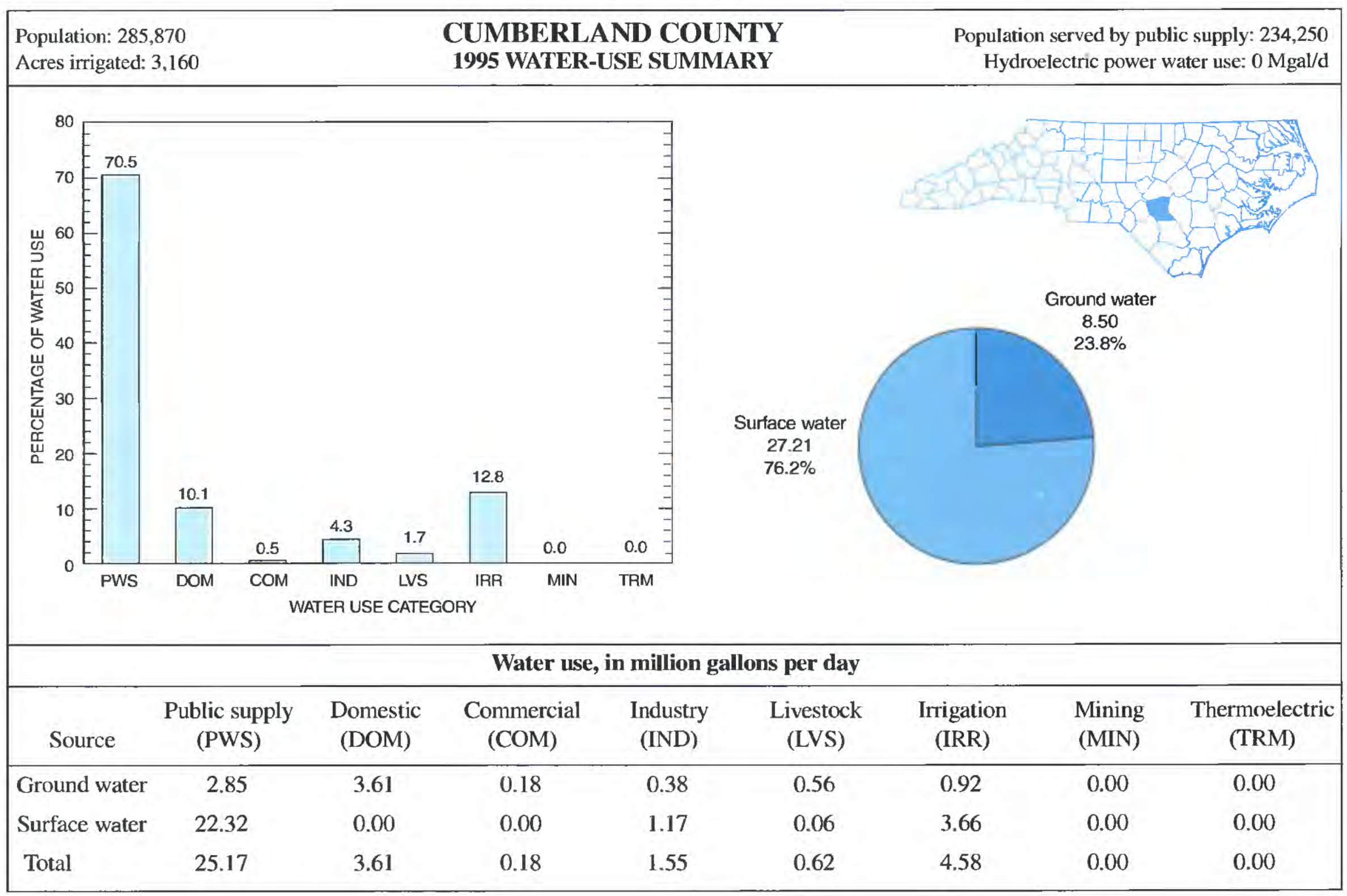




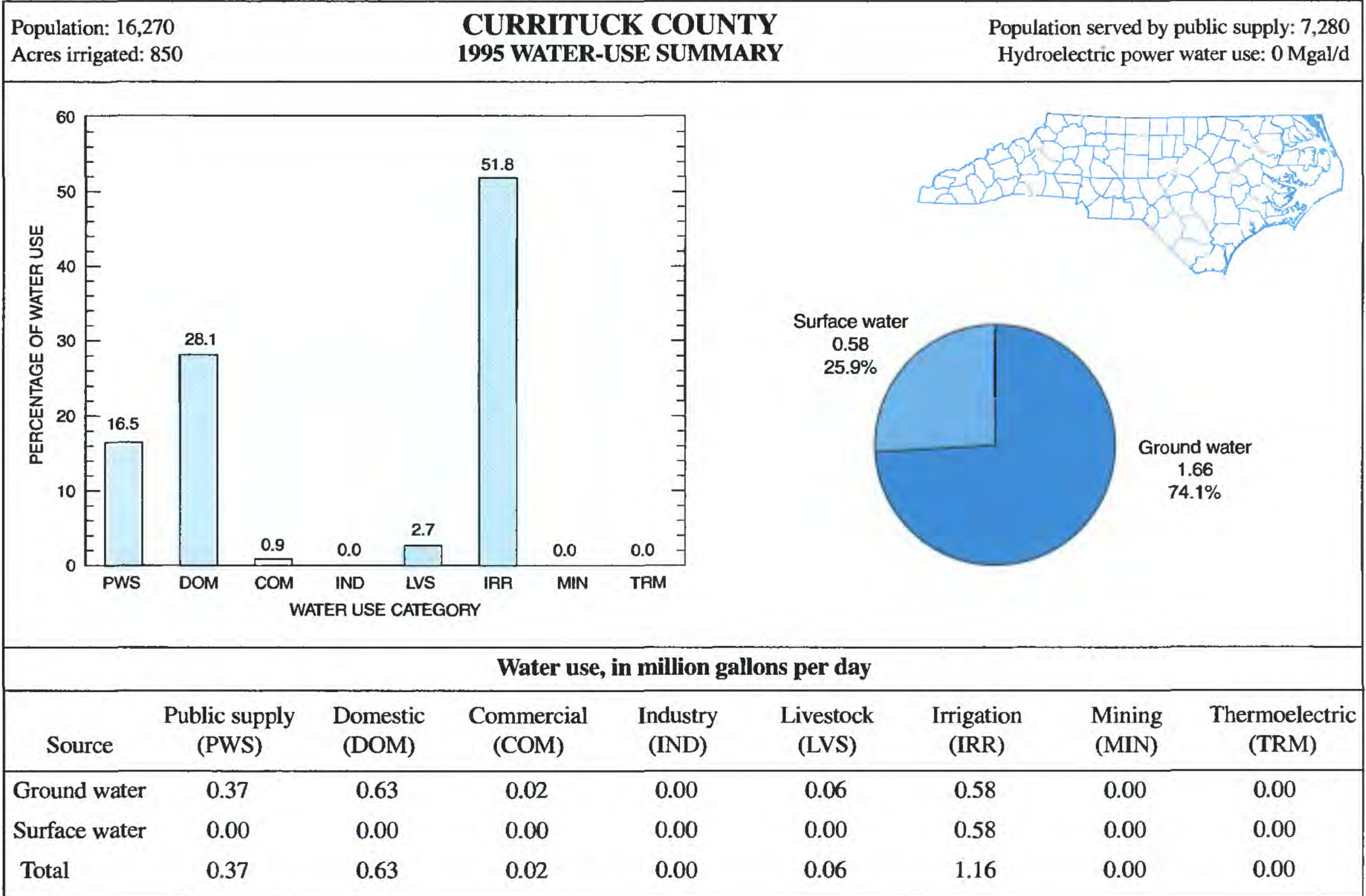

\begin{tabular}{|l|l}
$\begin{array}{l}\text { Population: } 26,050 \\
\text { Acres irrigated: } 450\end{array}$ & DARE COUNTY \\
\hline
\end{tabular}




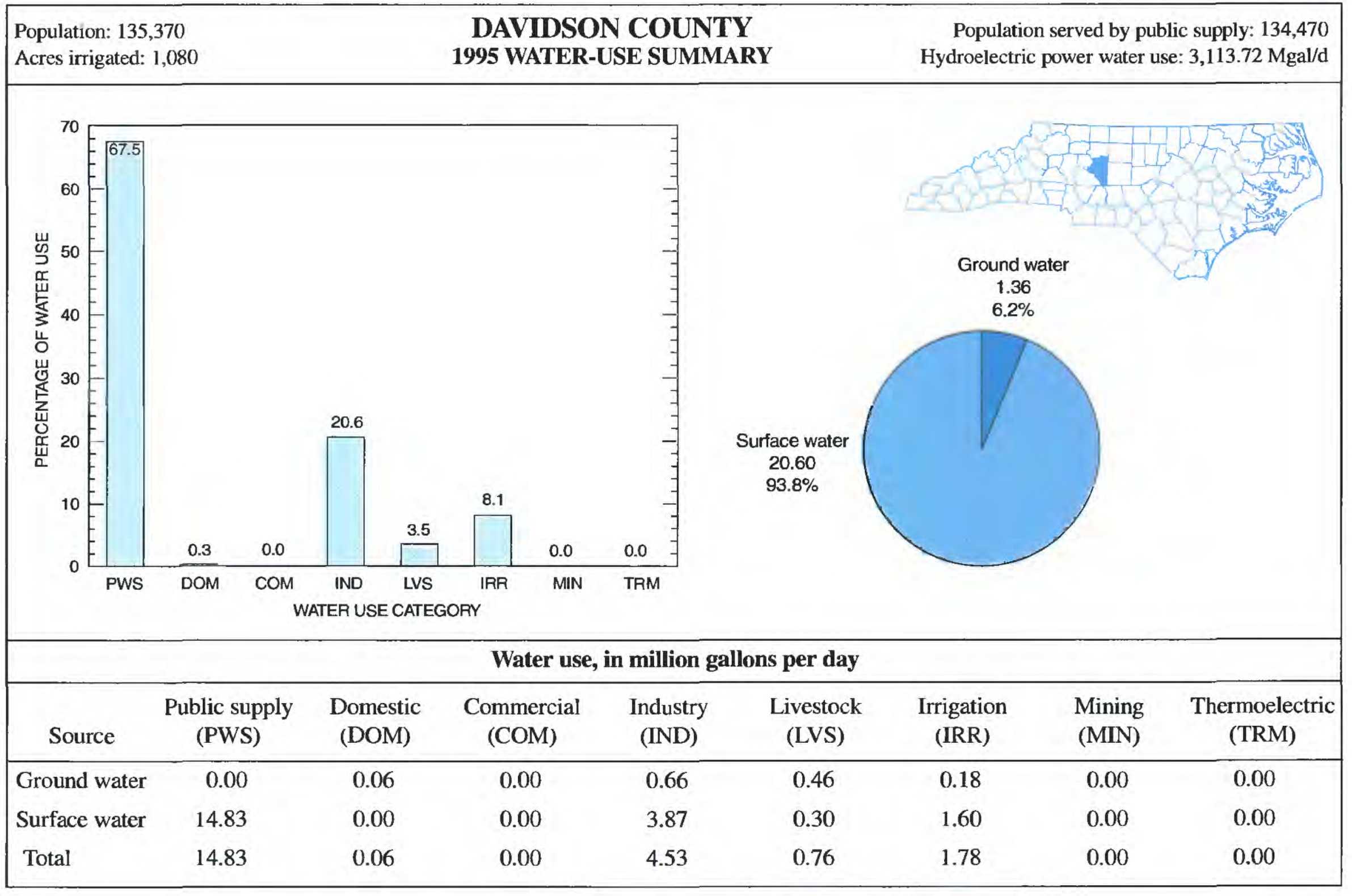

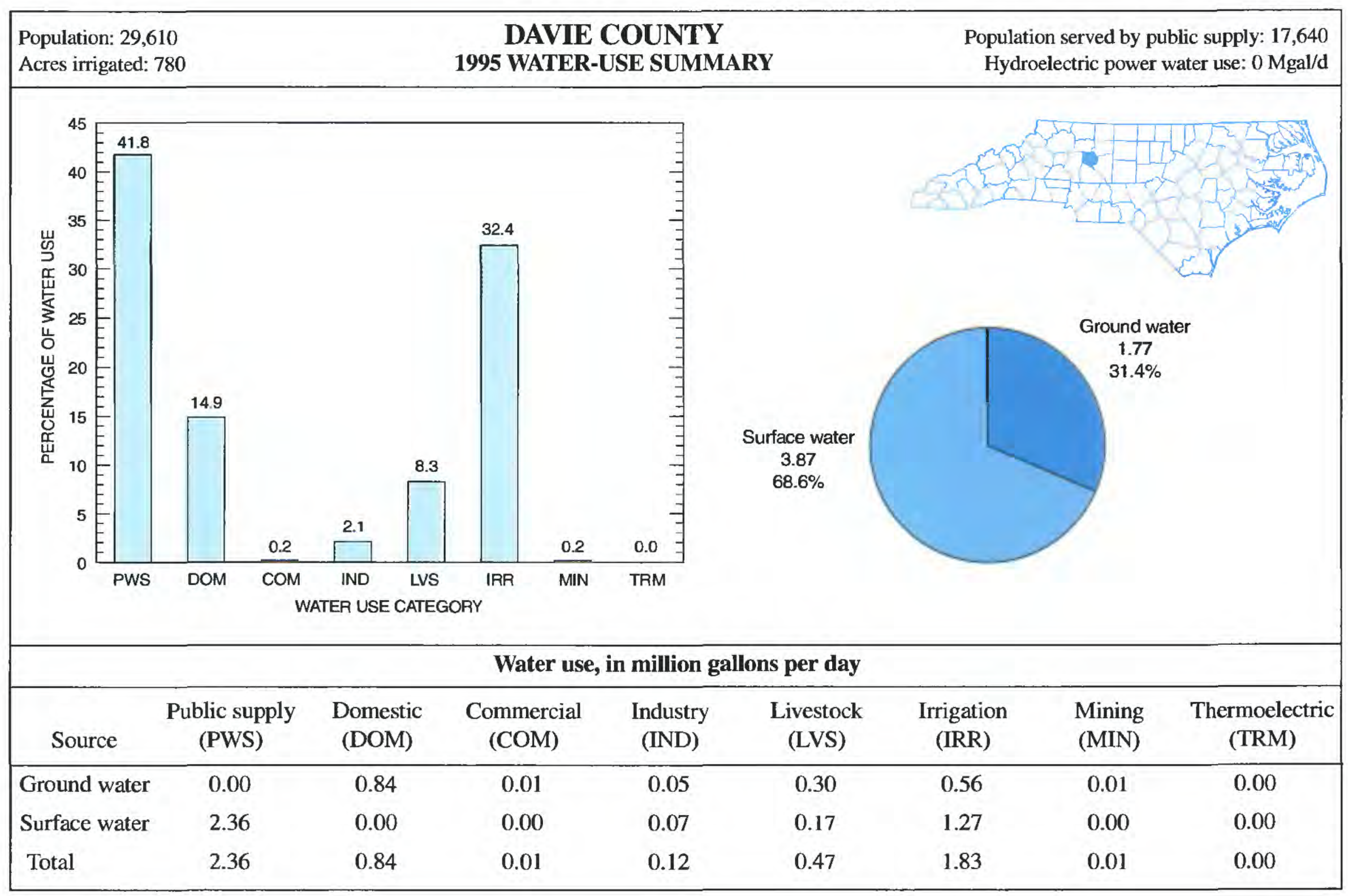




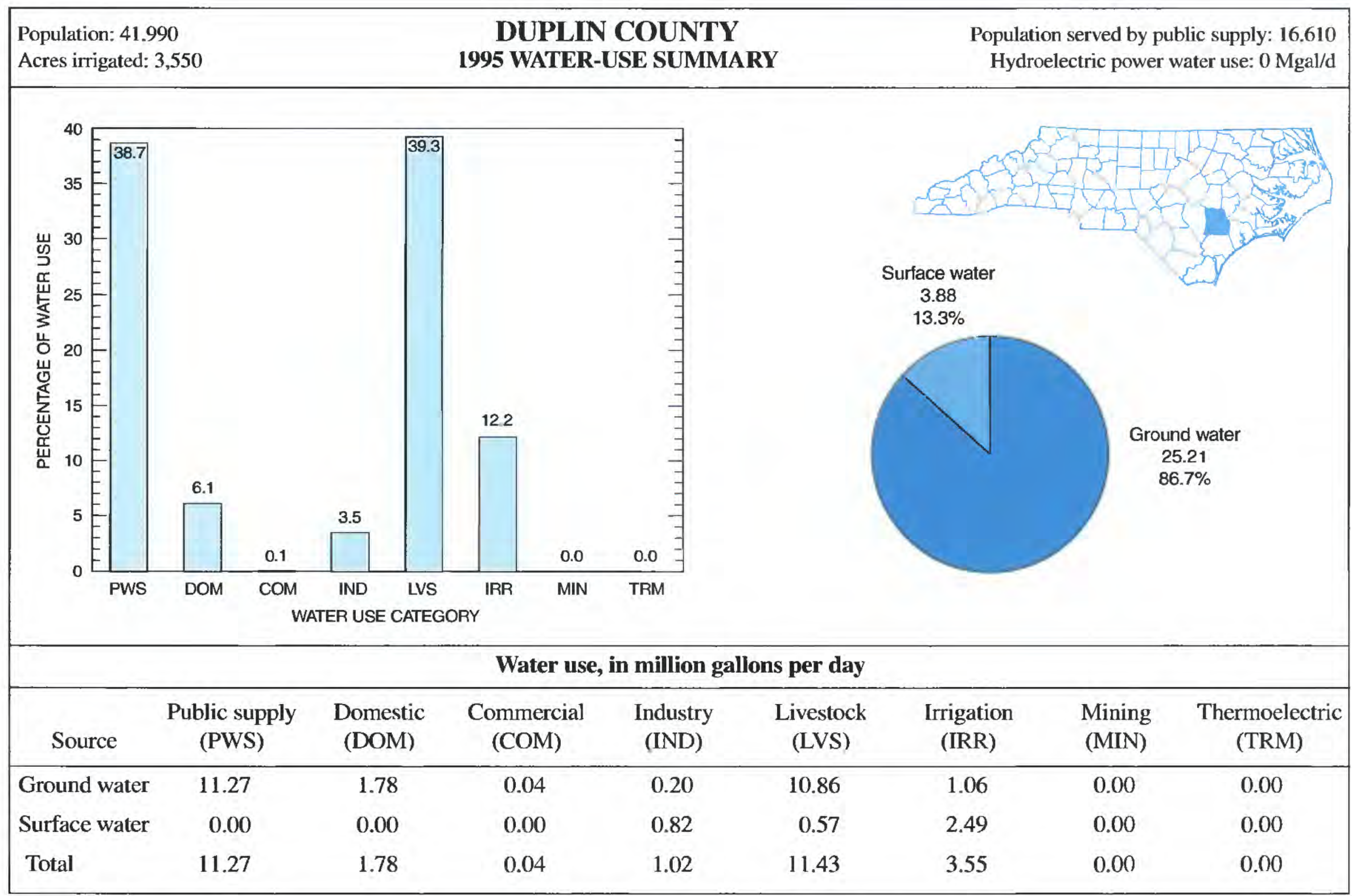

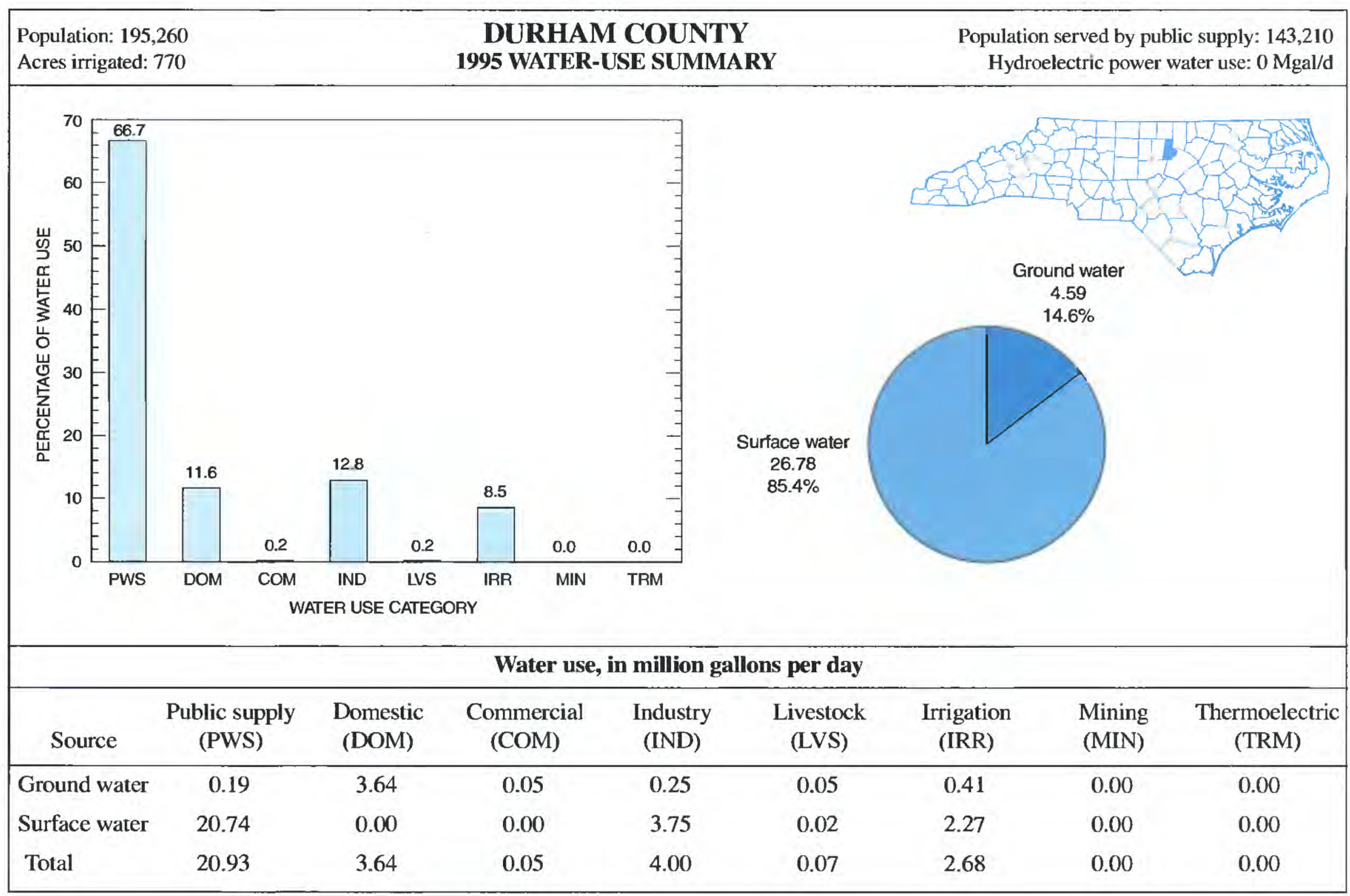




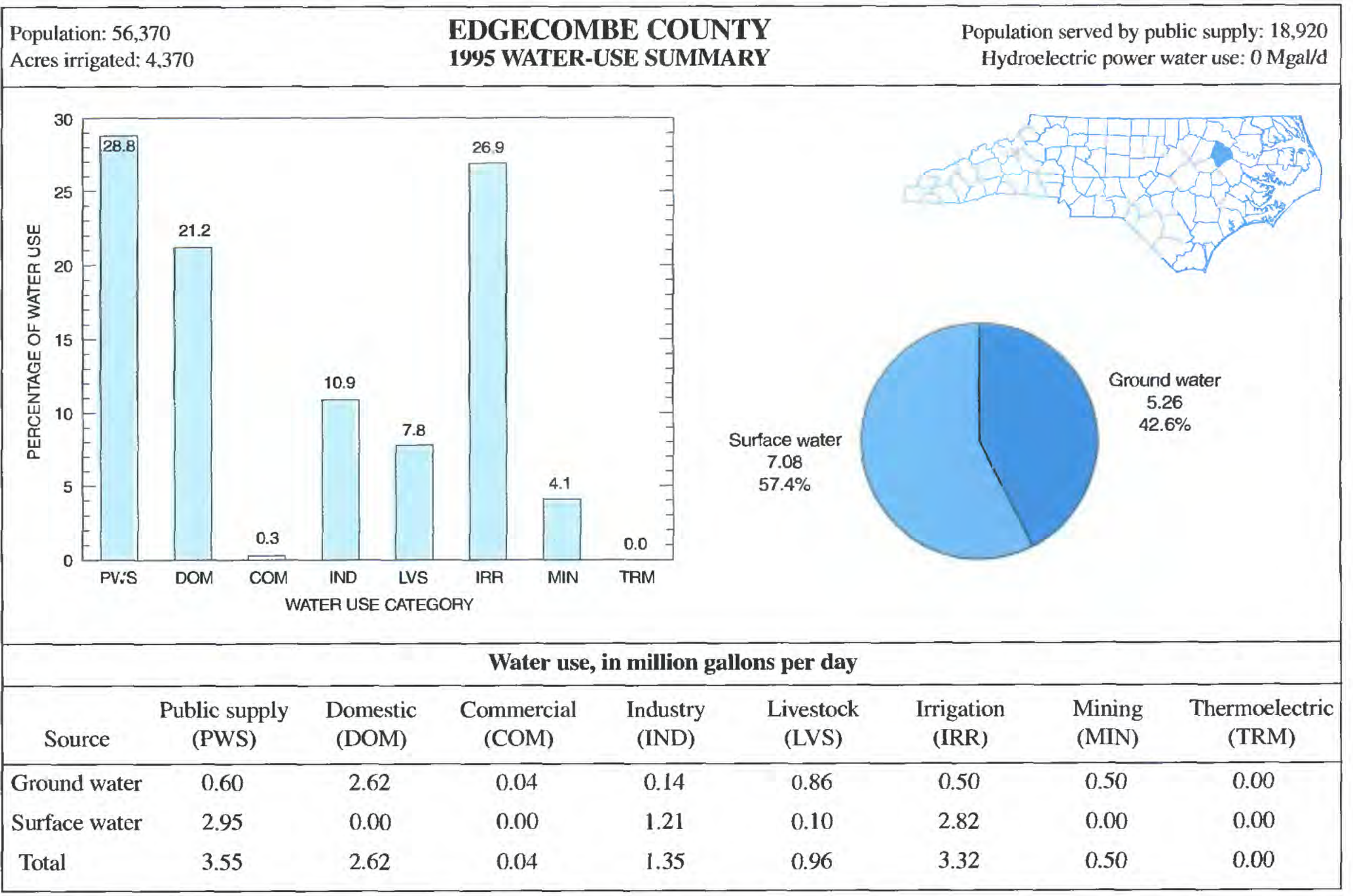

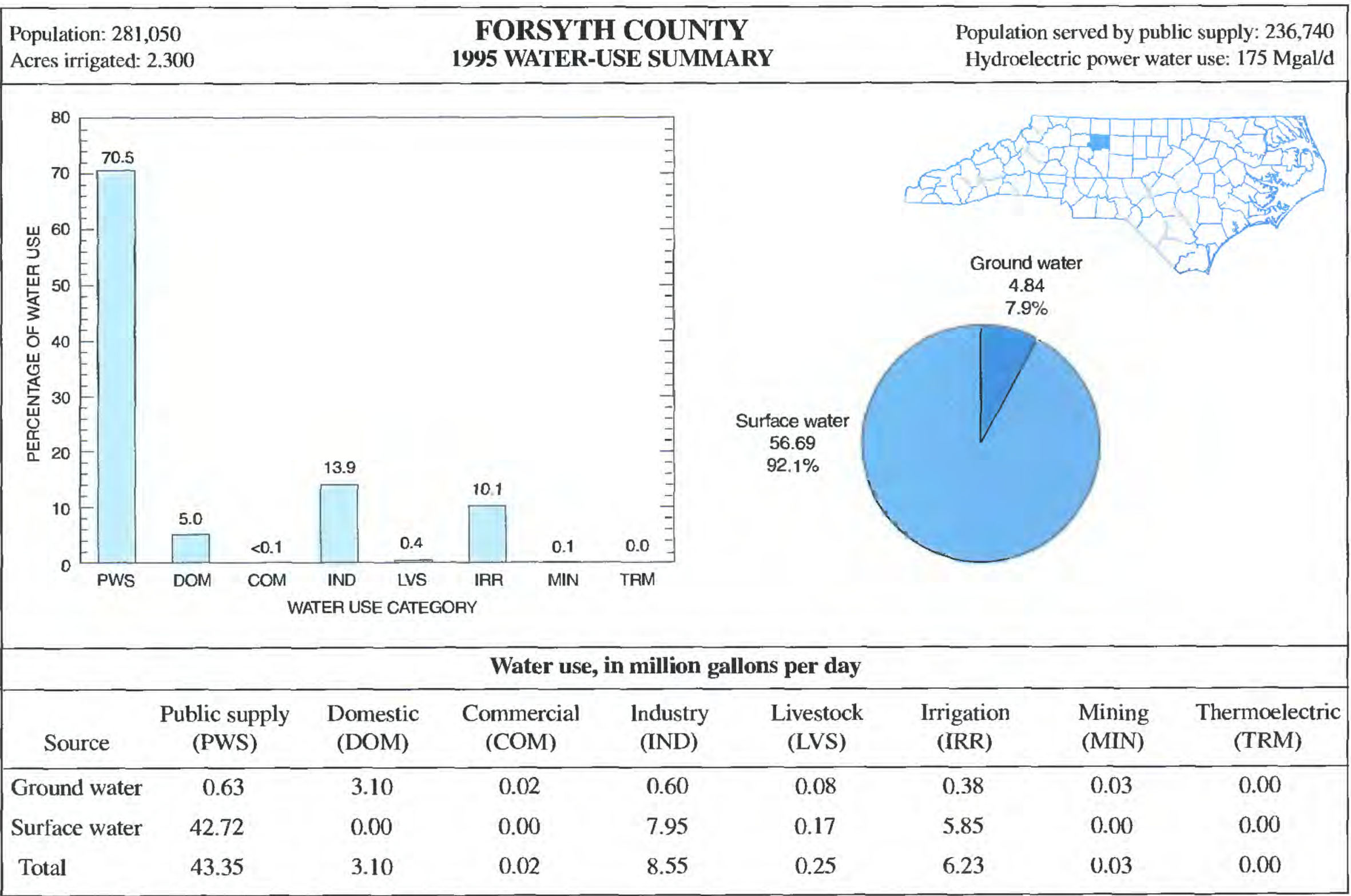




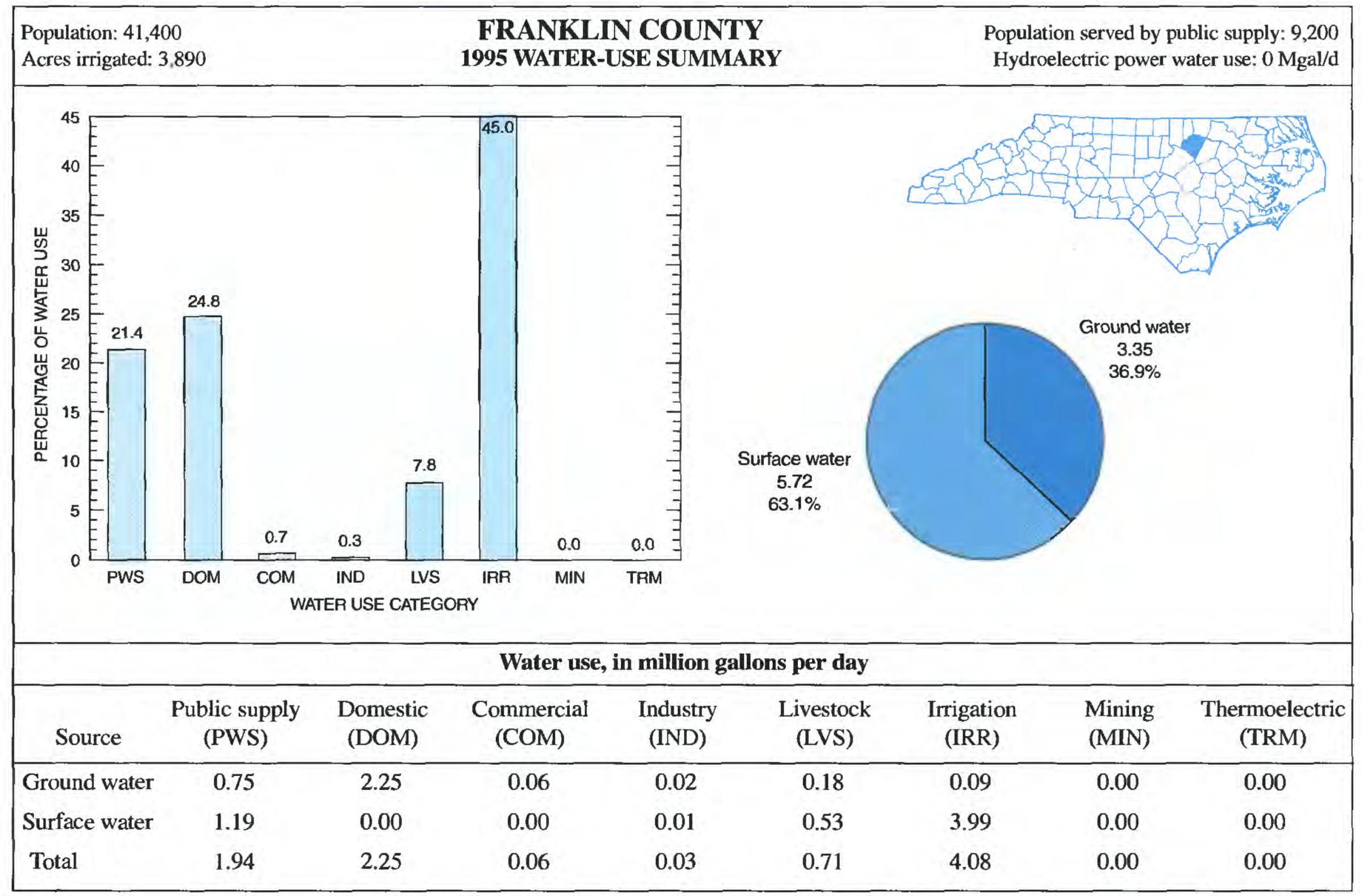

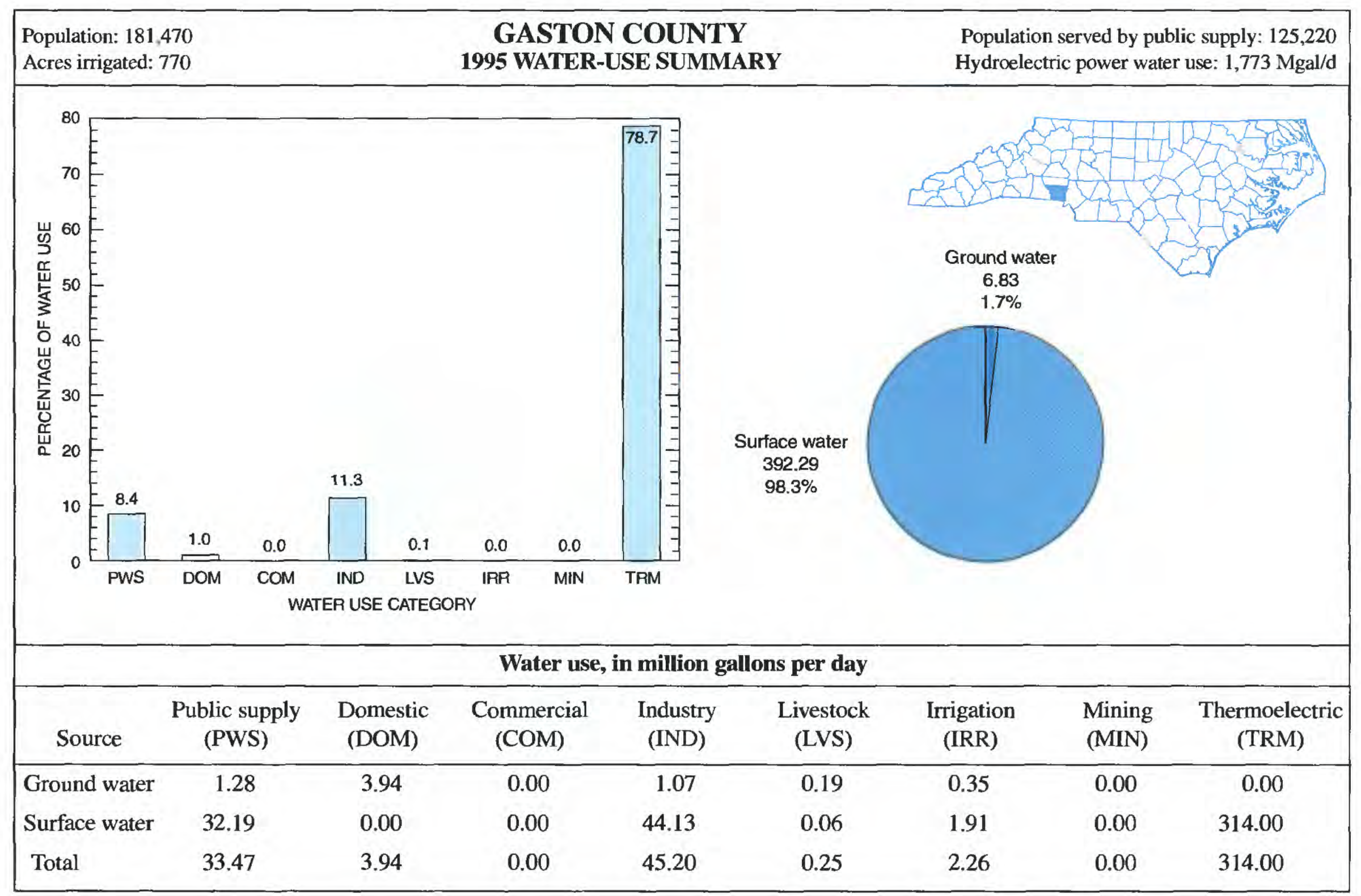




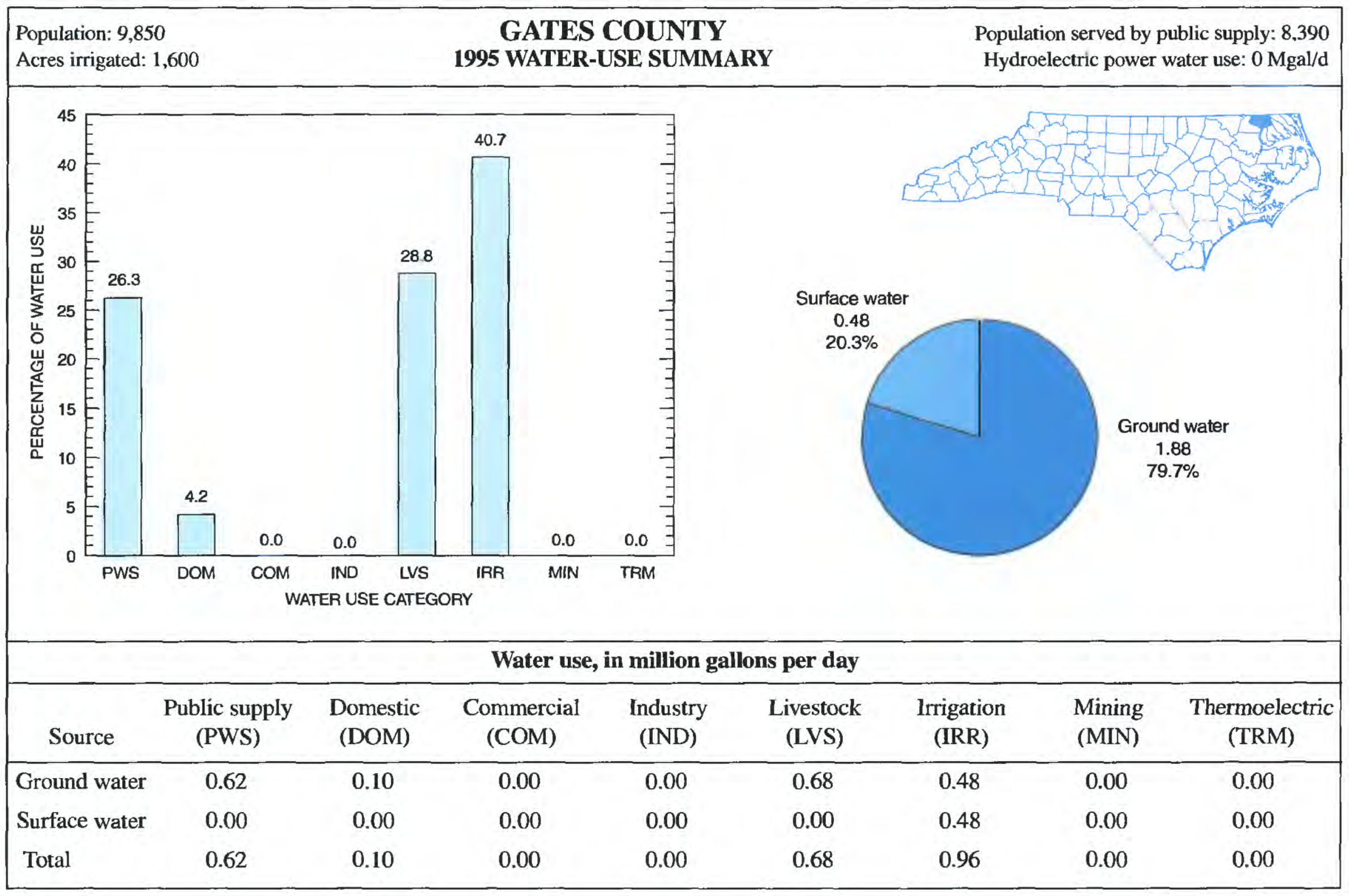

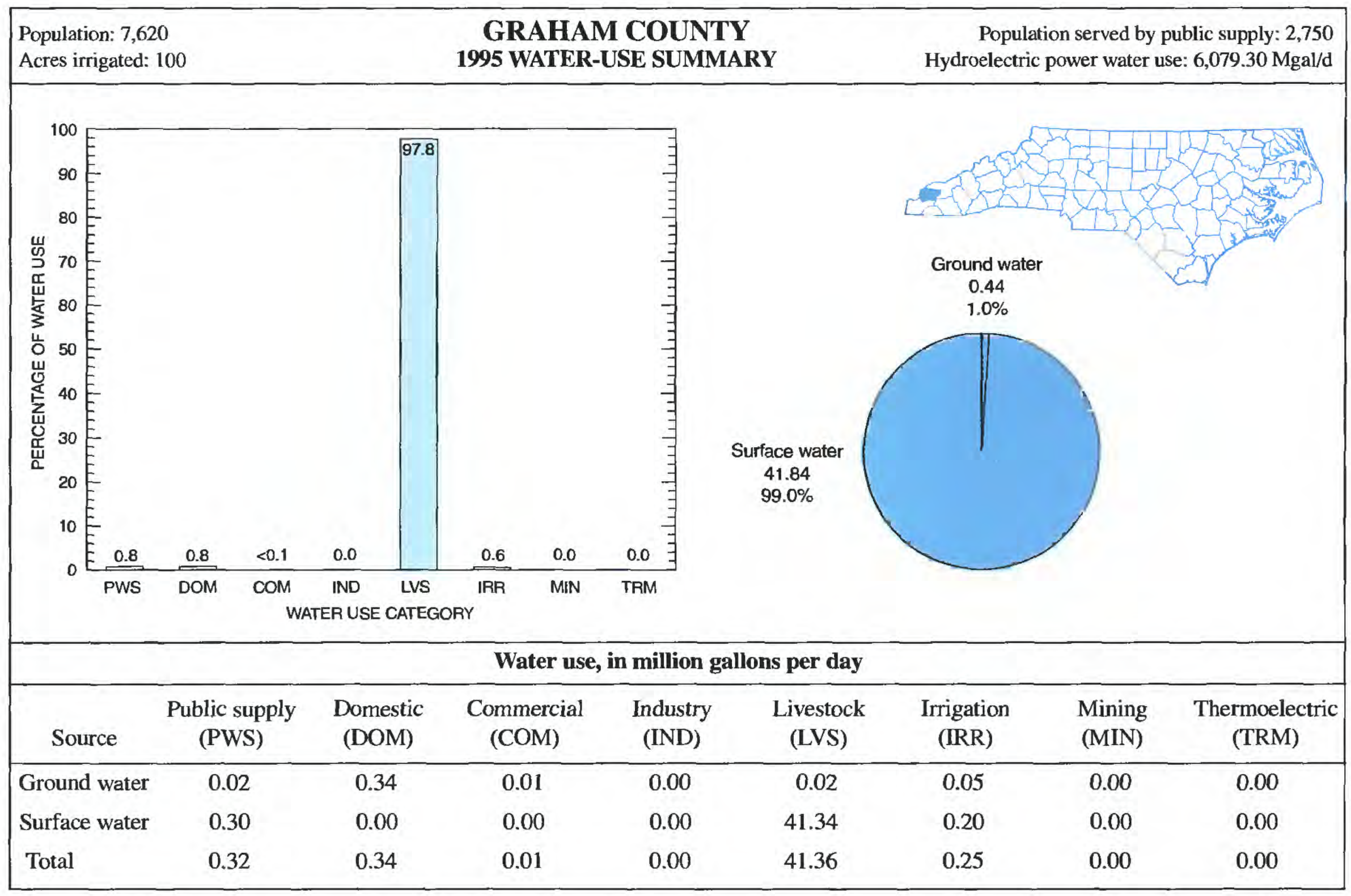




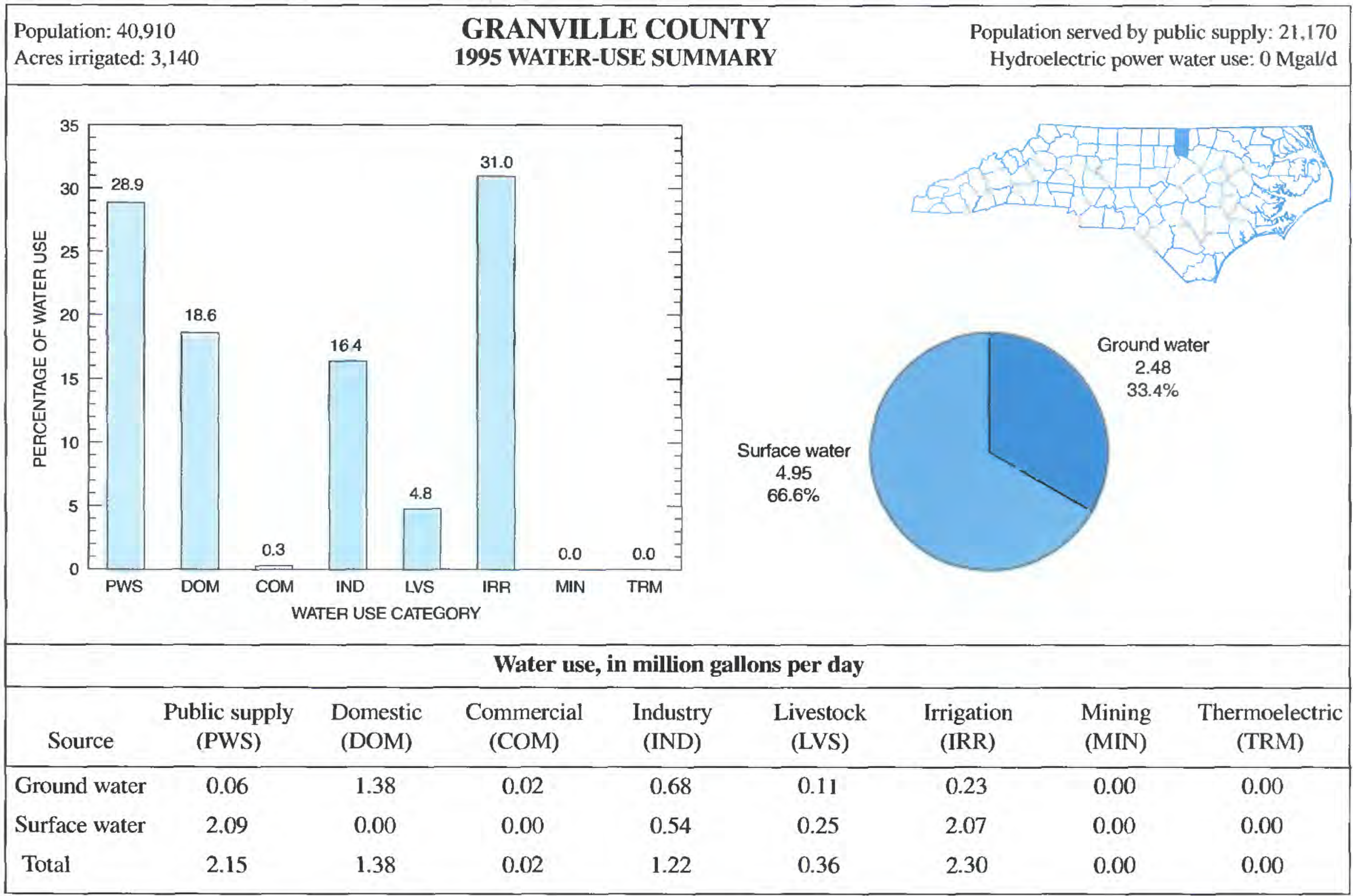

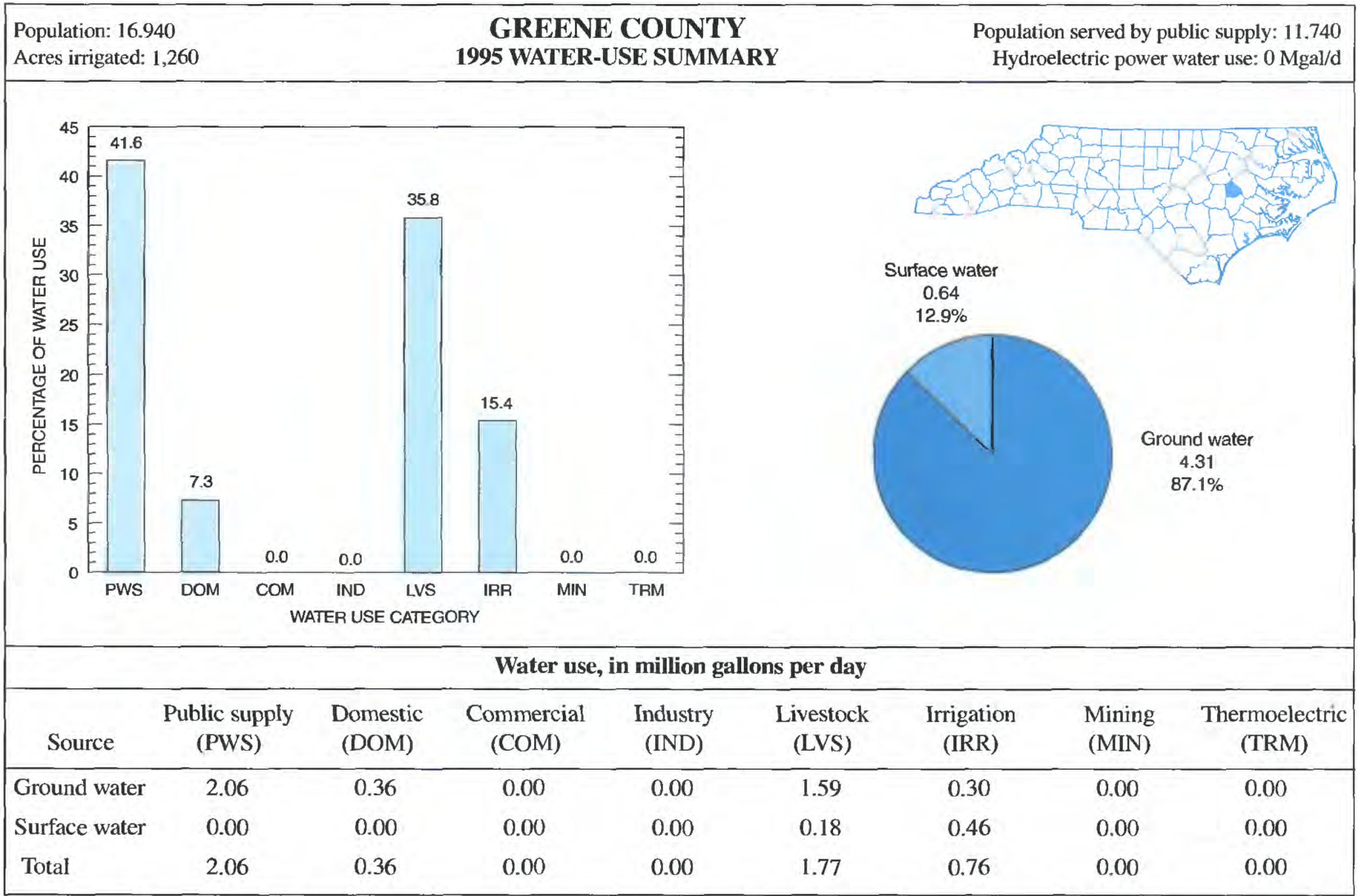




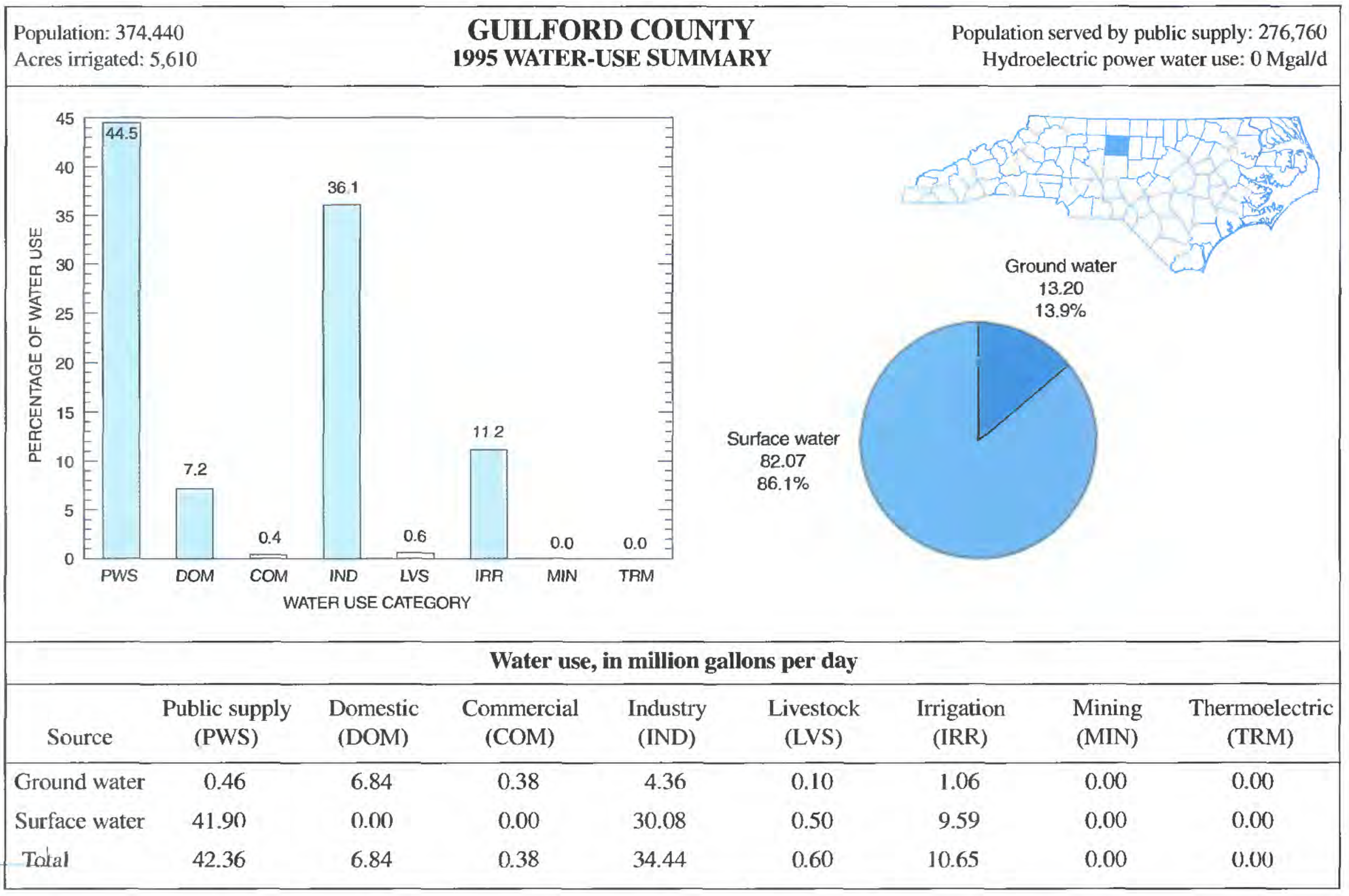

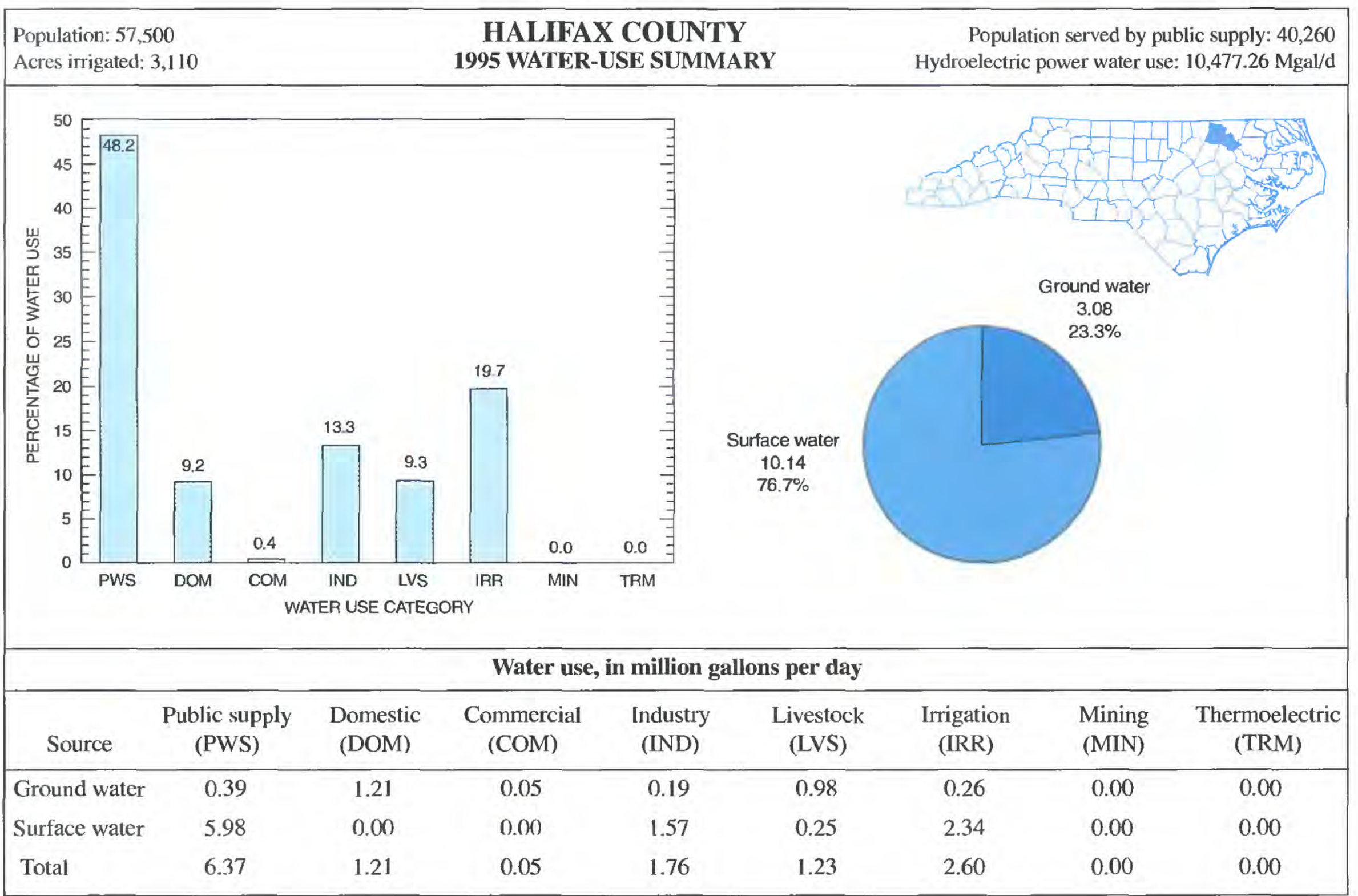




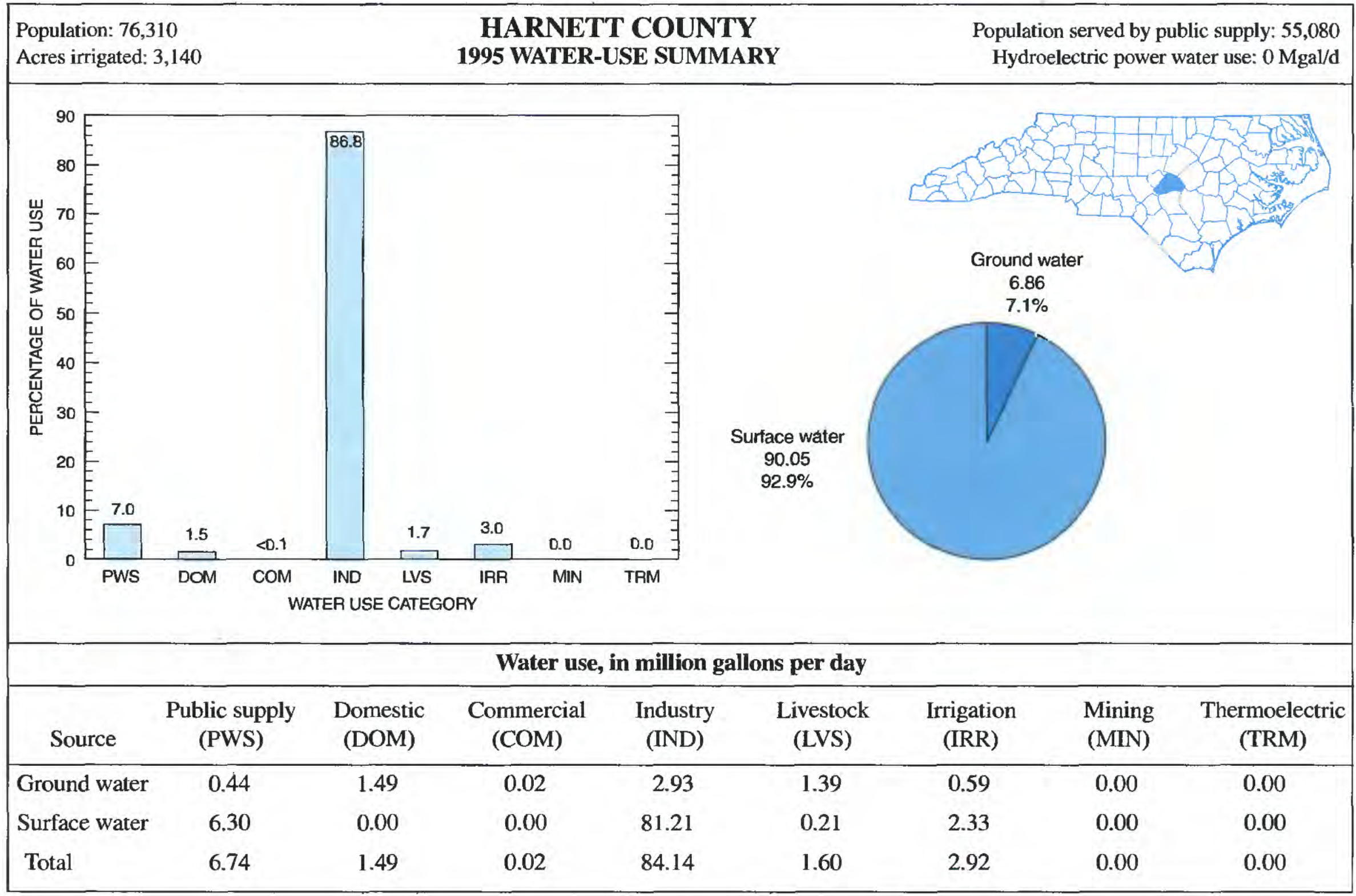

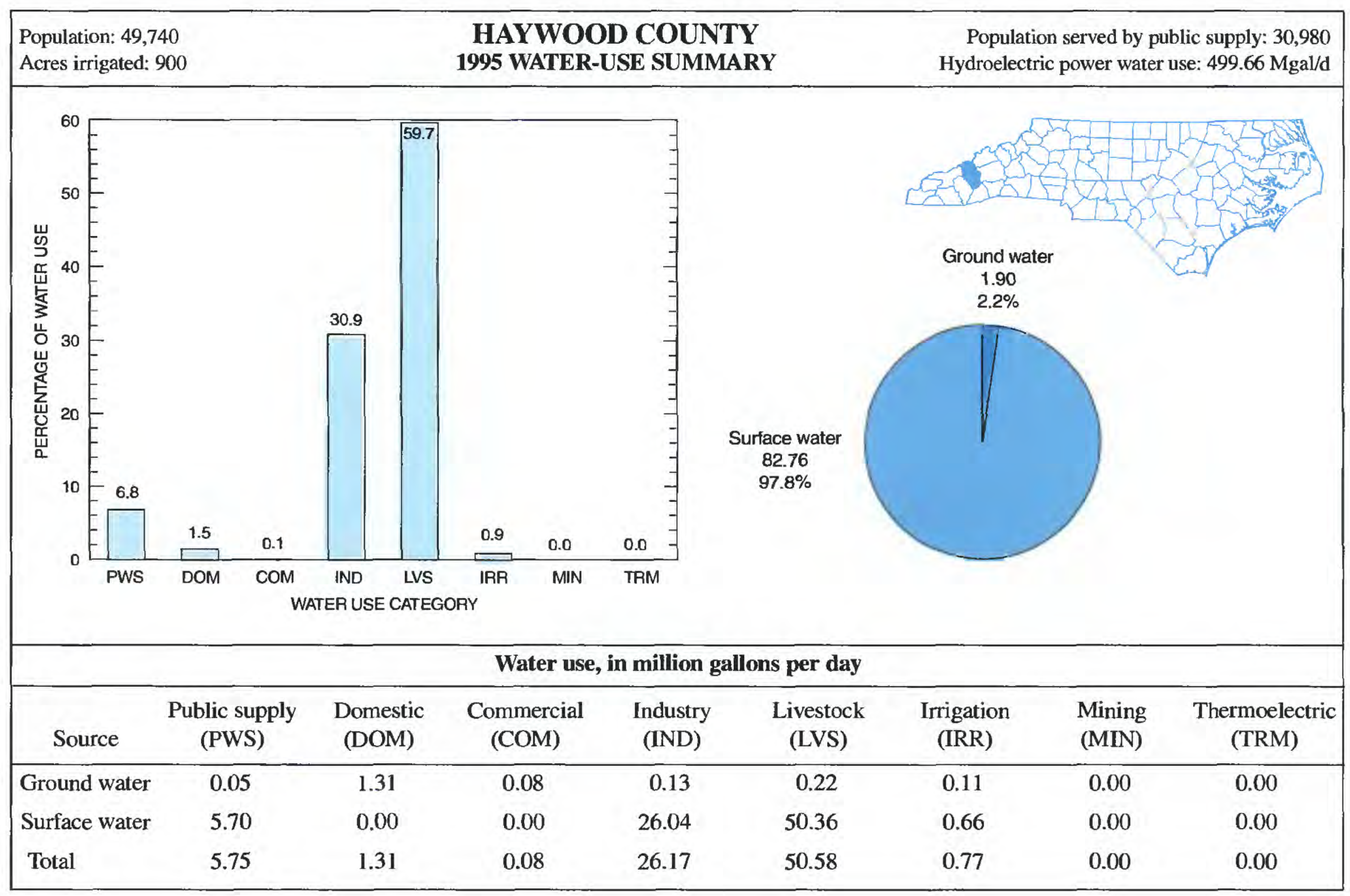




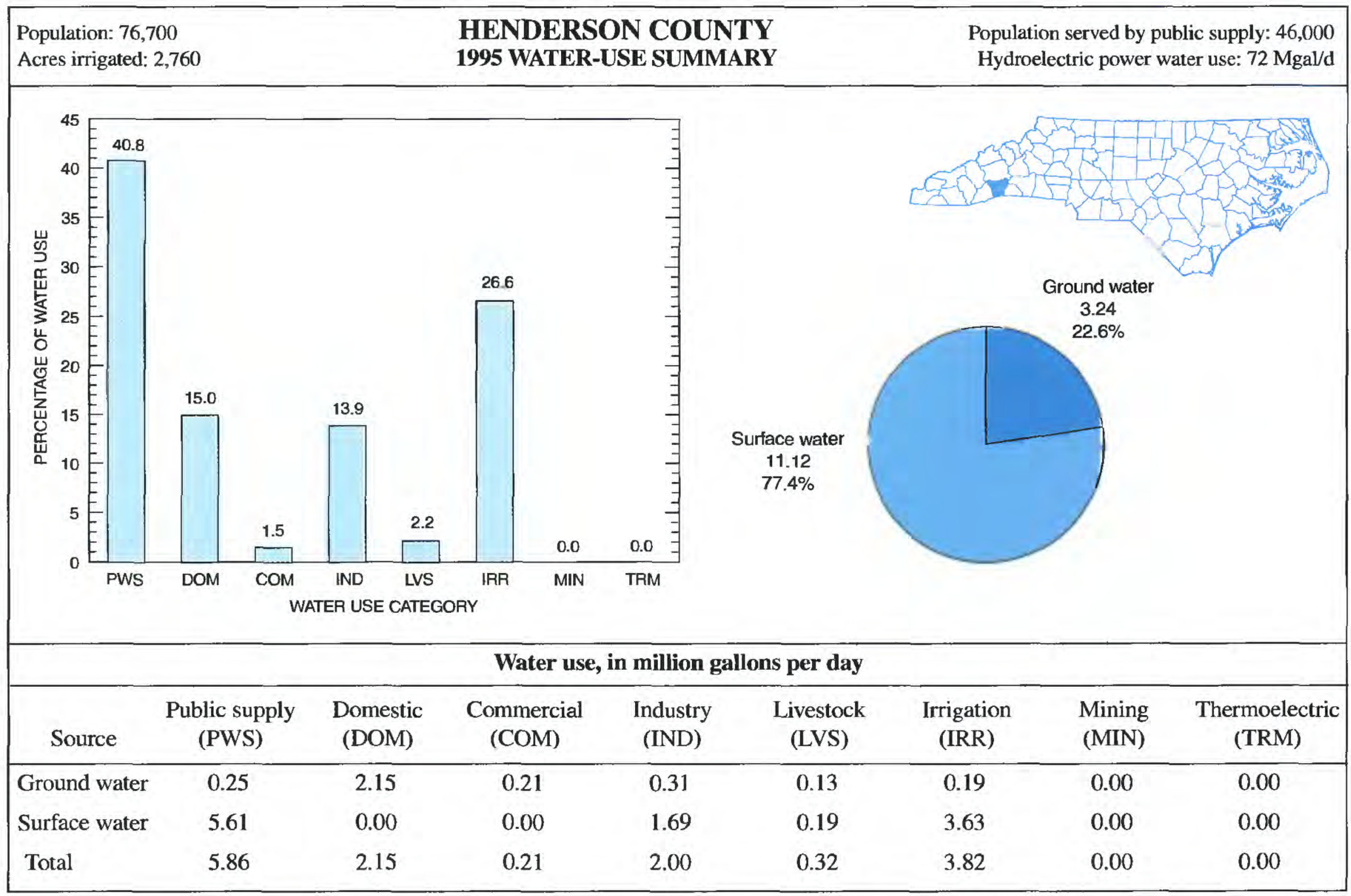

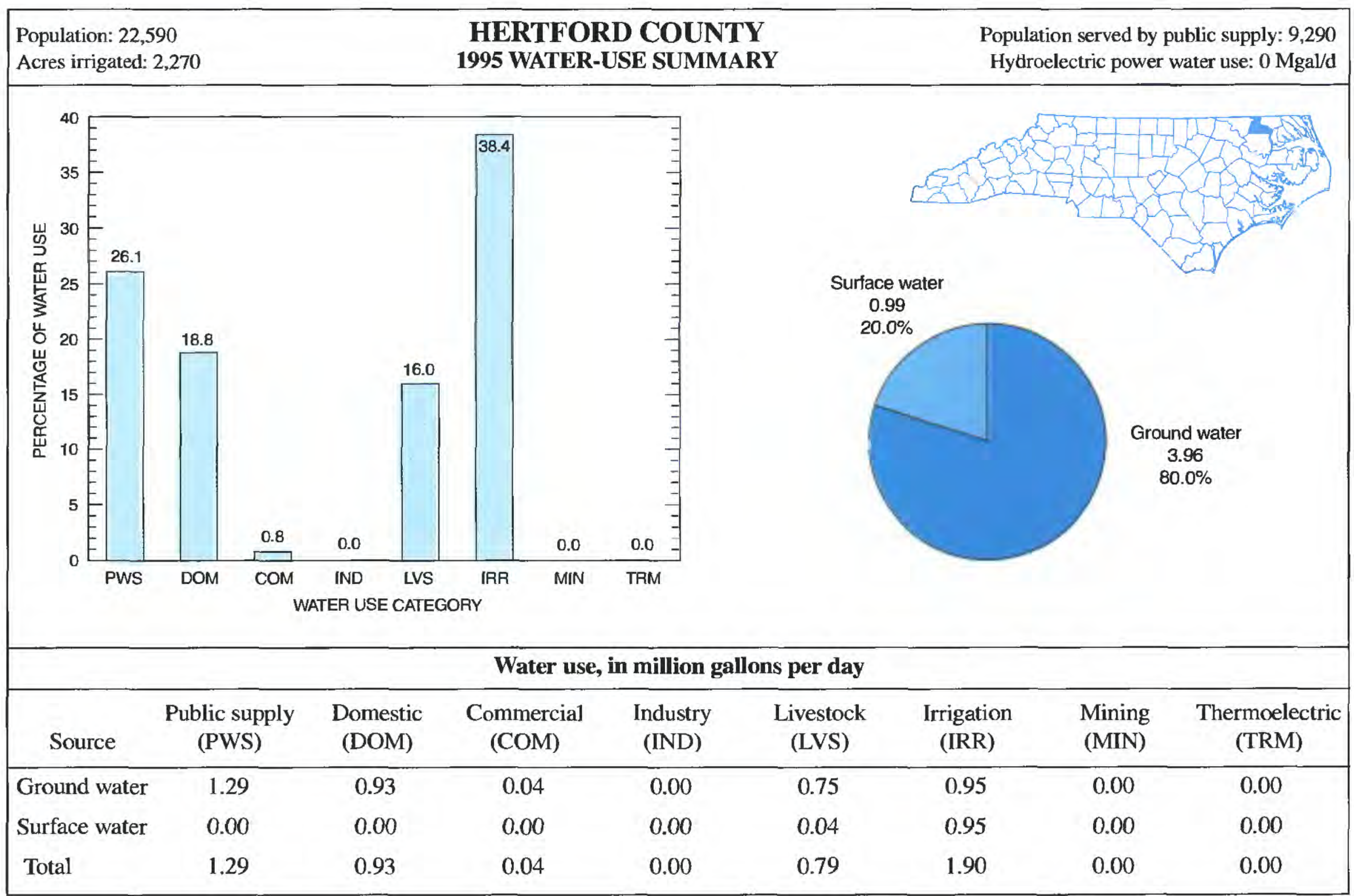




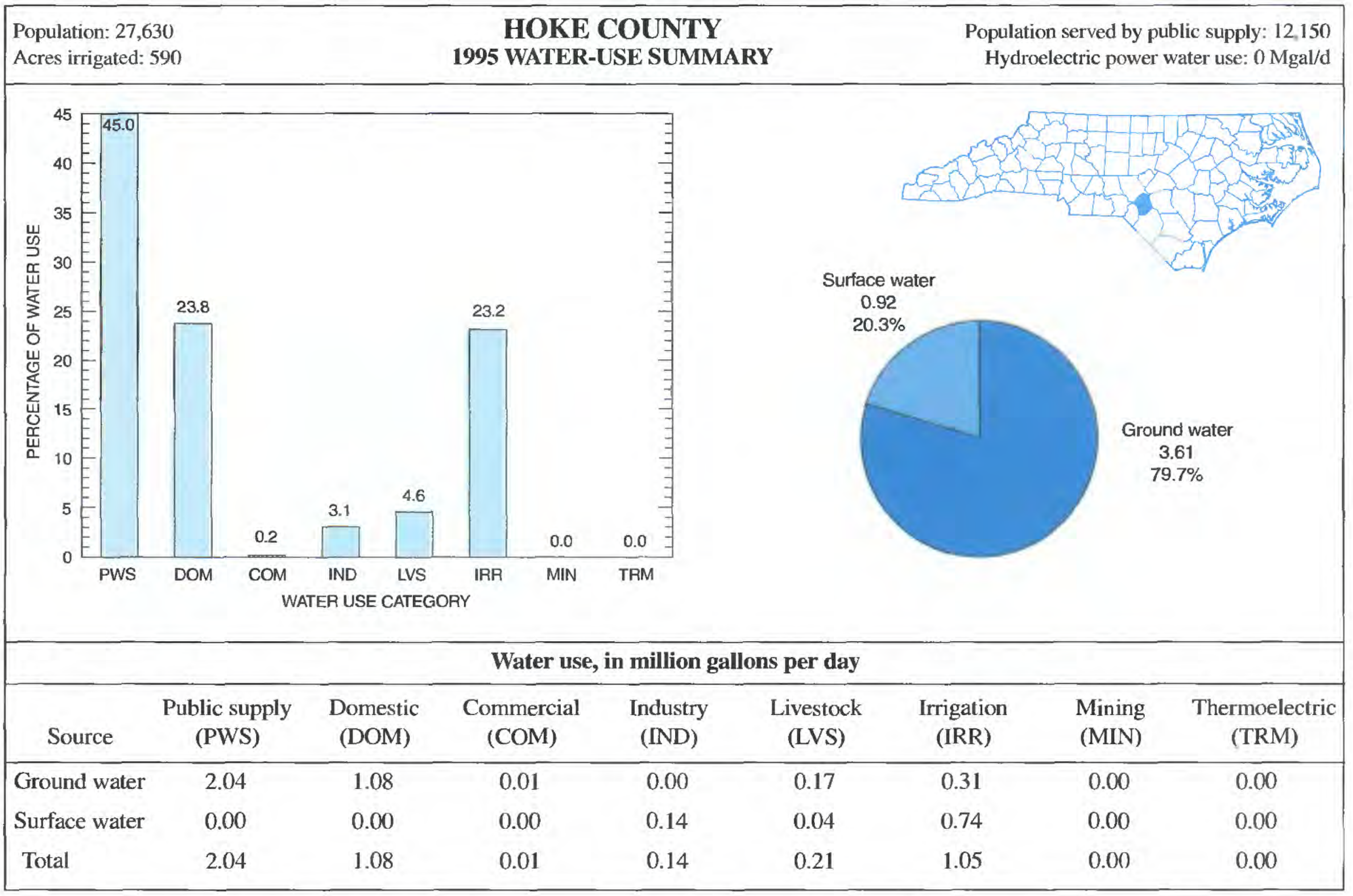

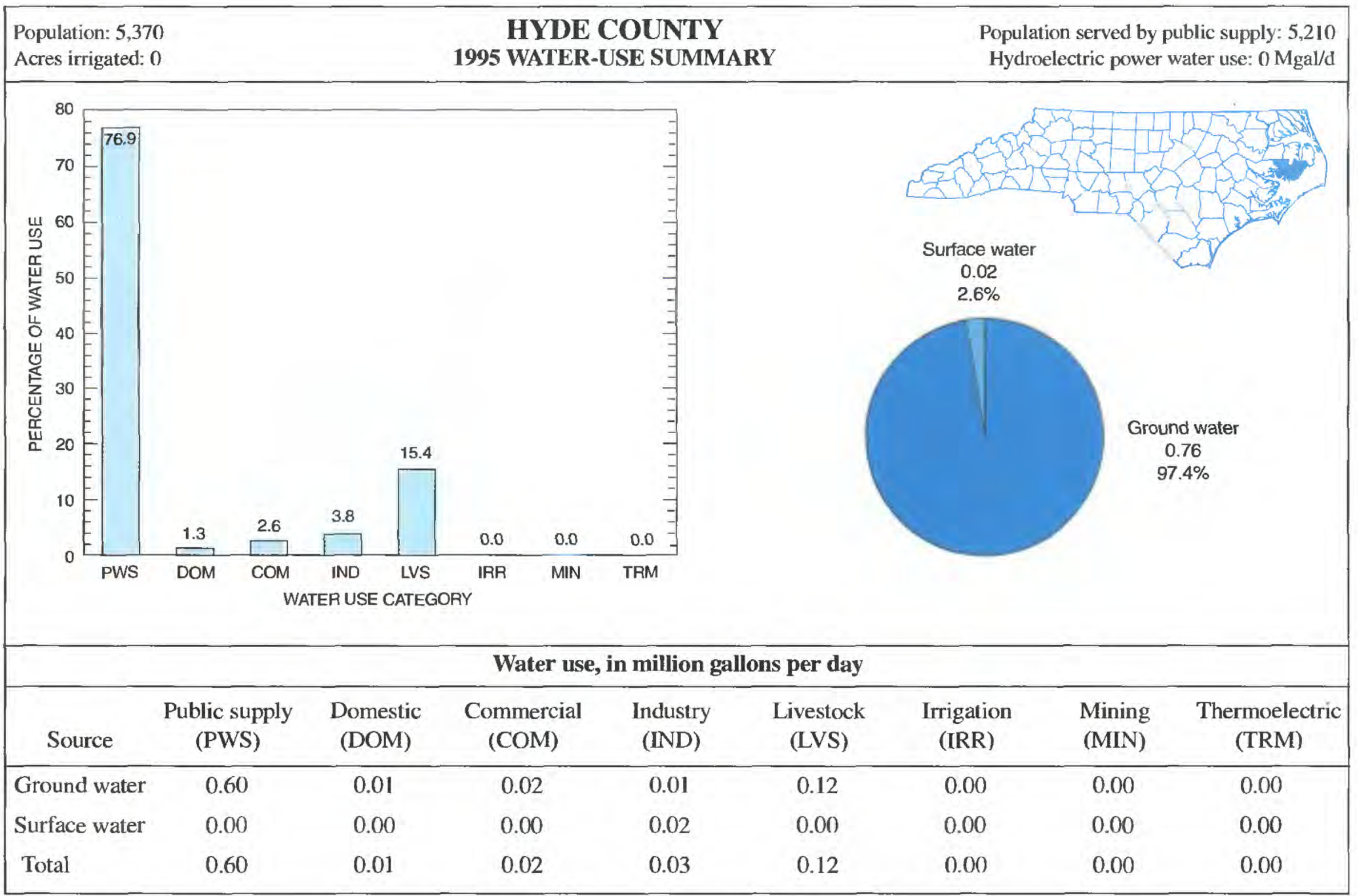




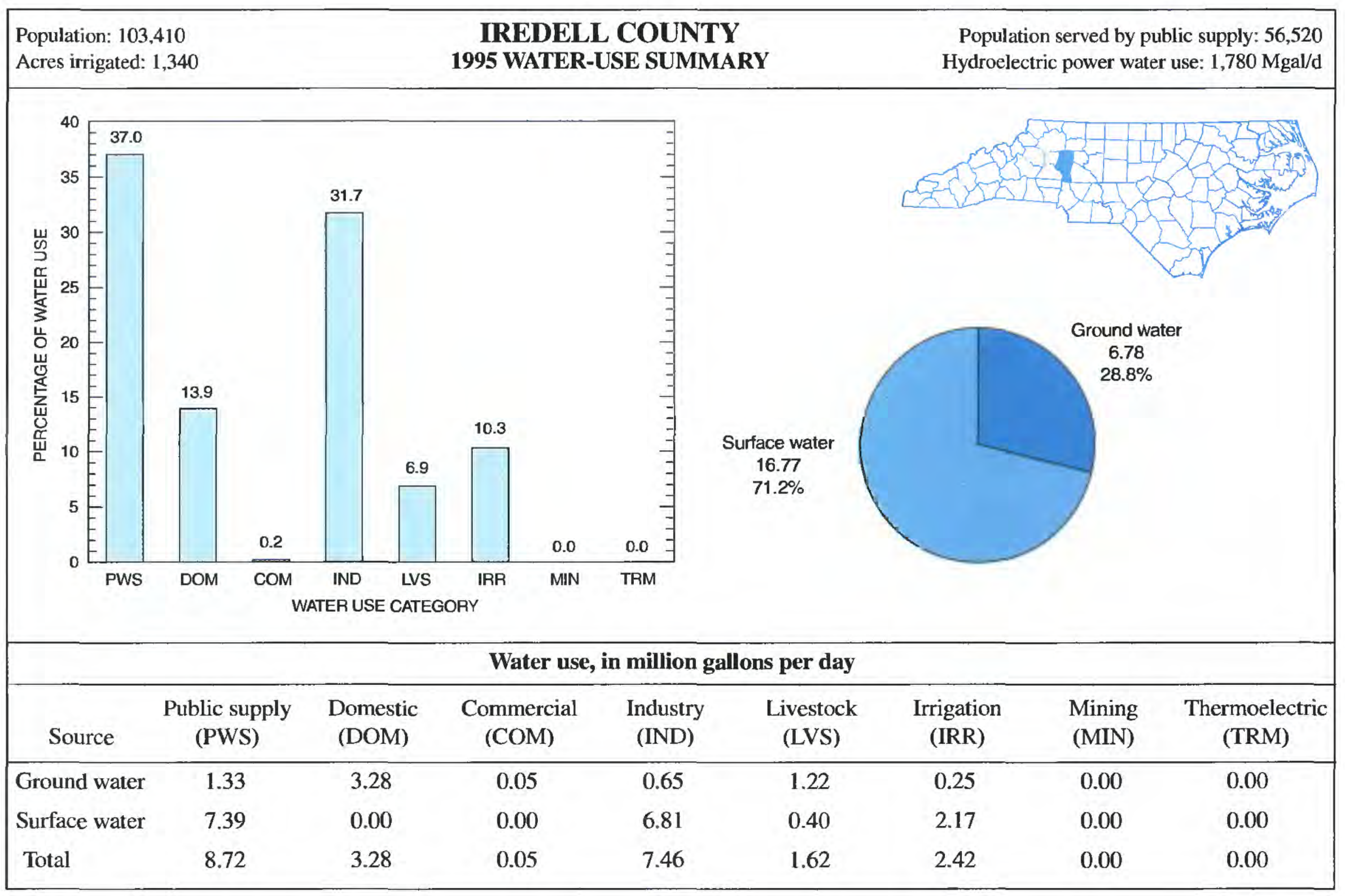

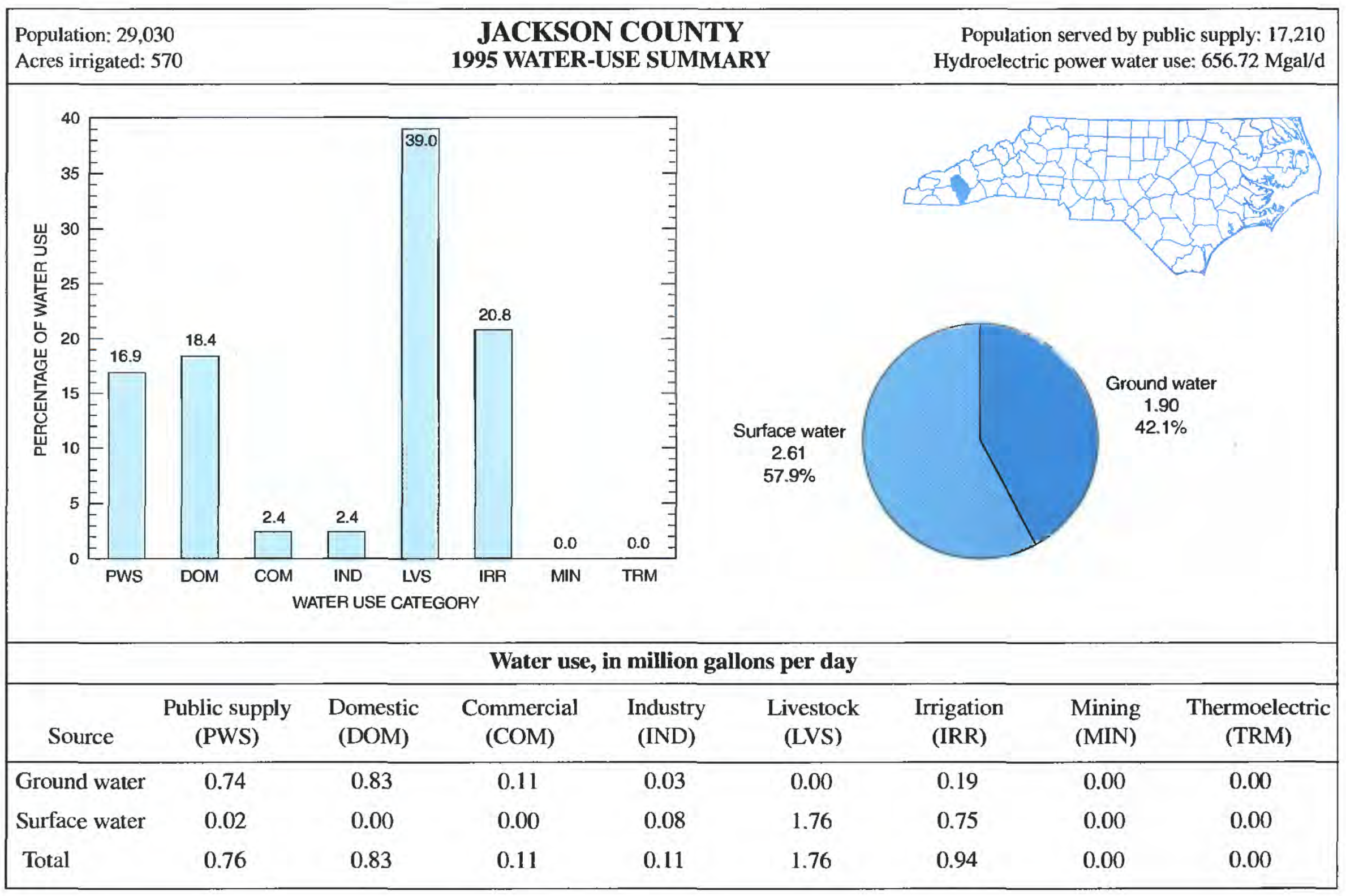




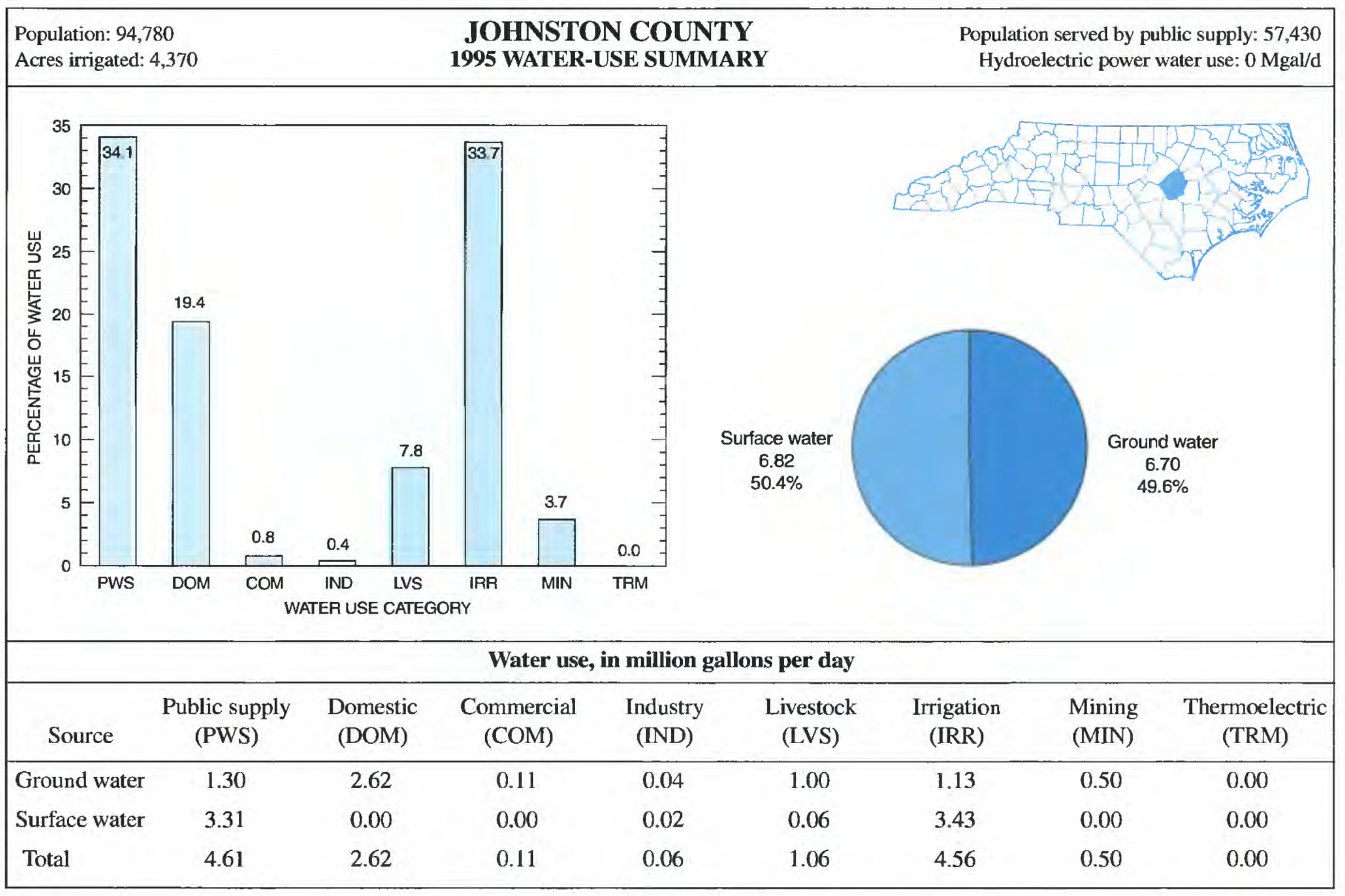

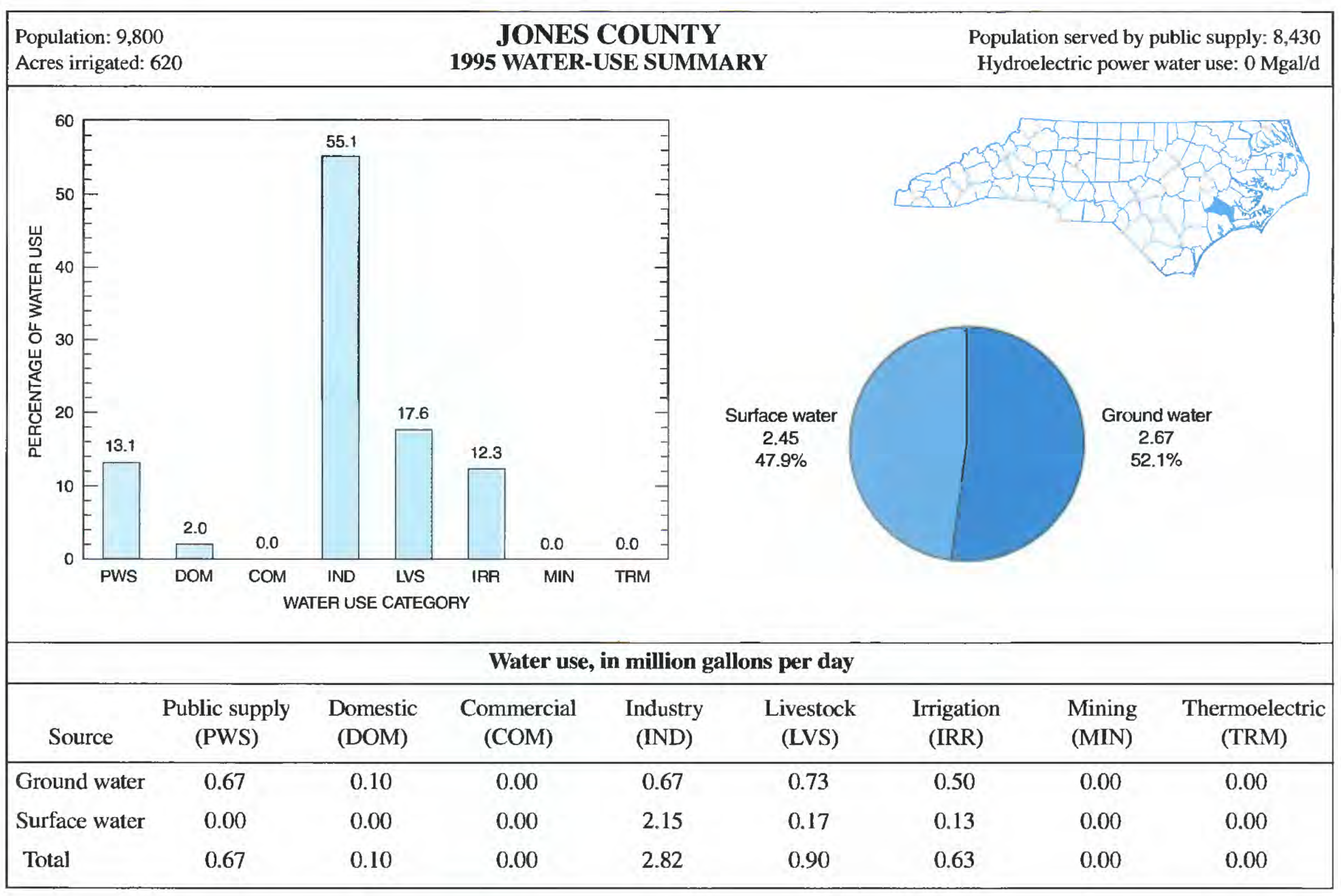




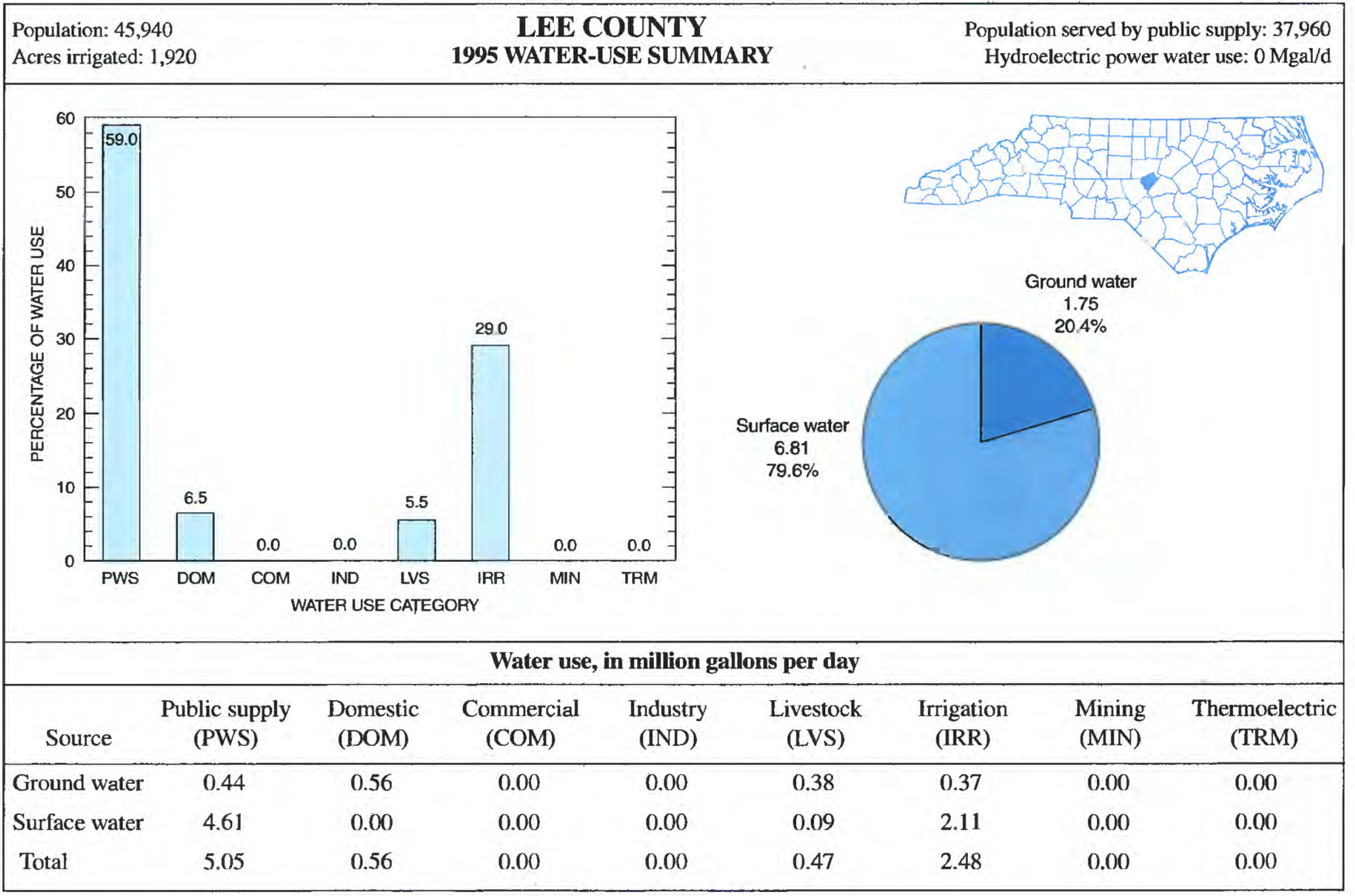

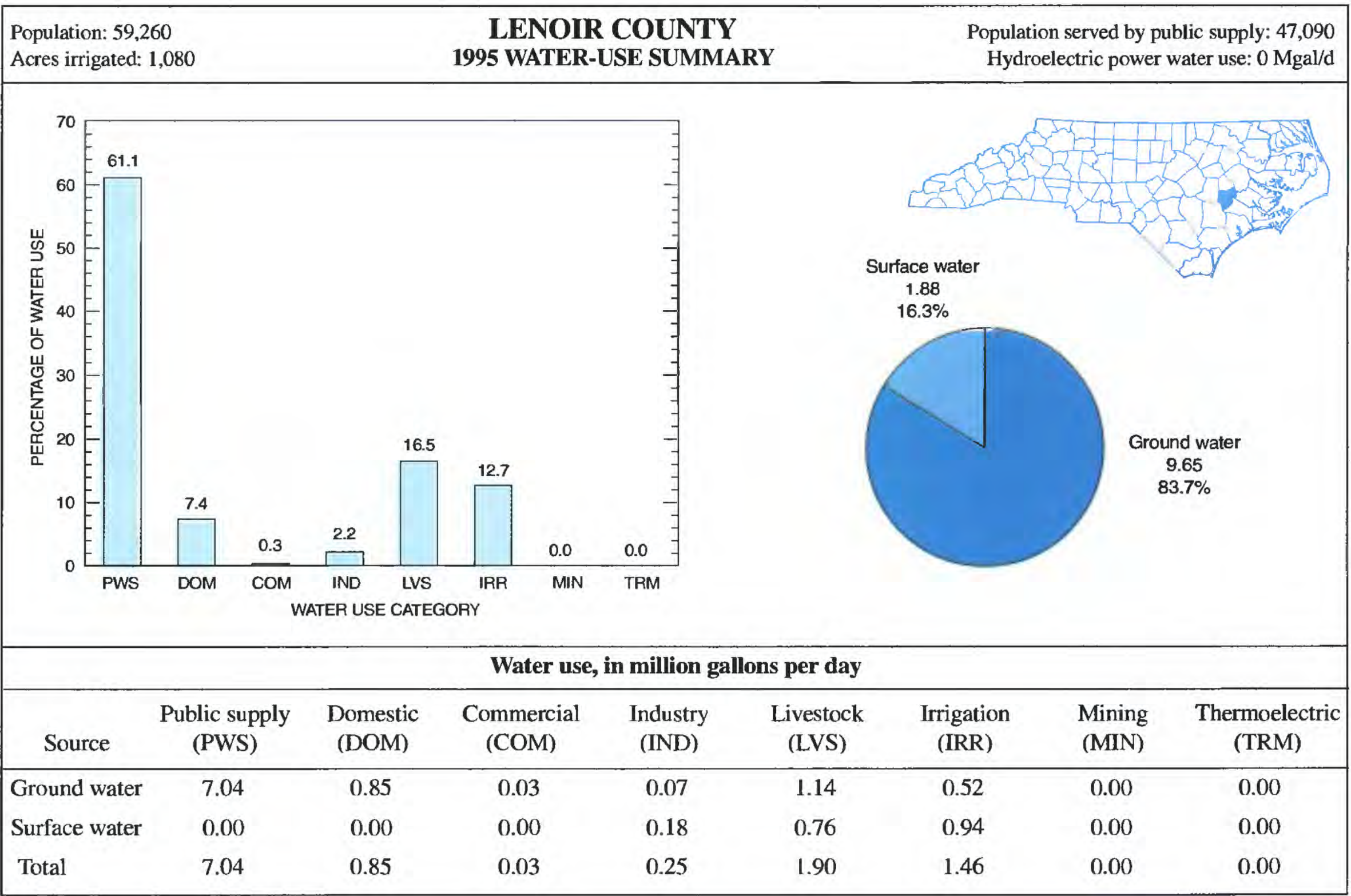




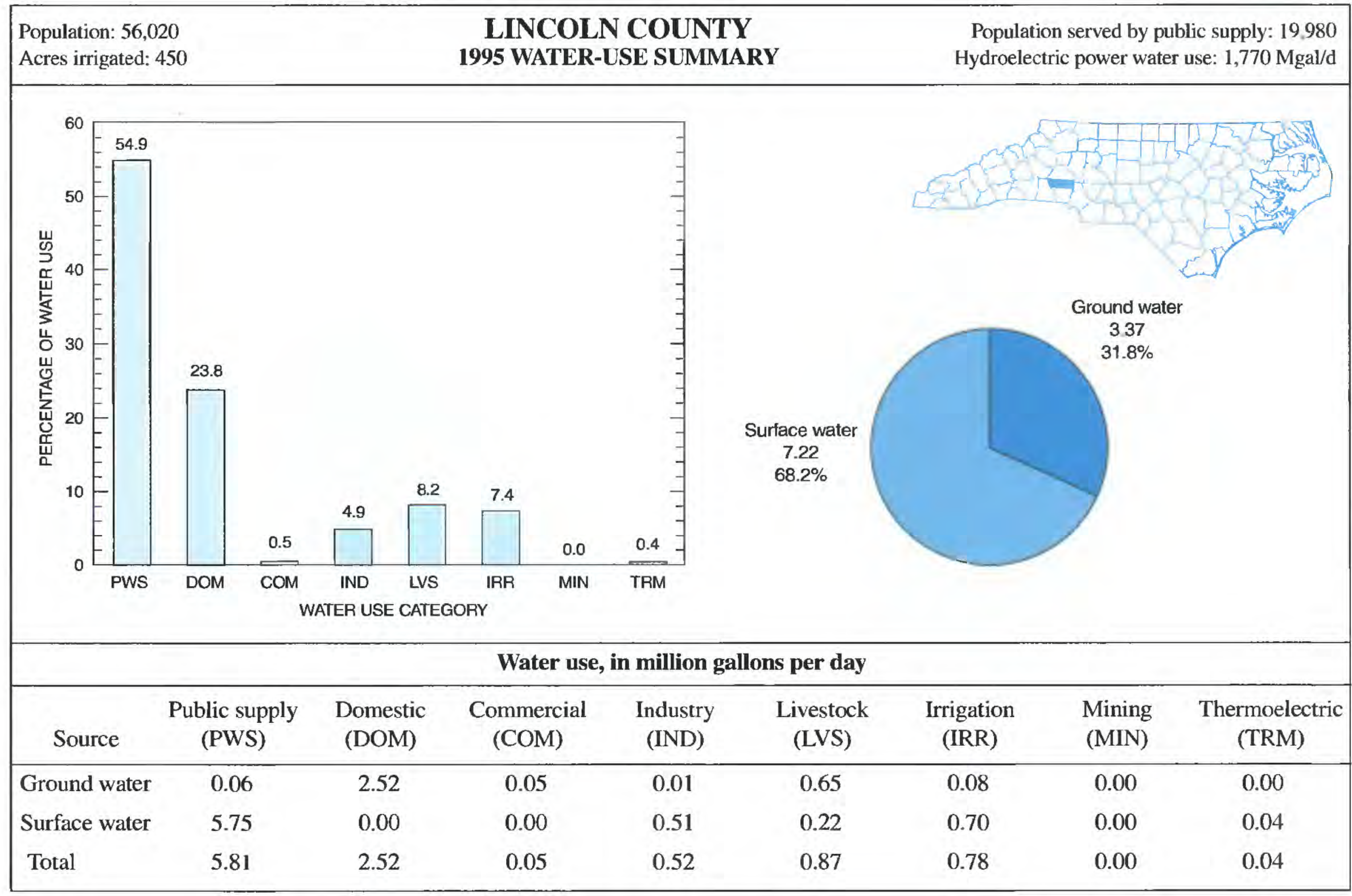

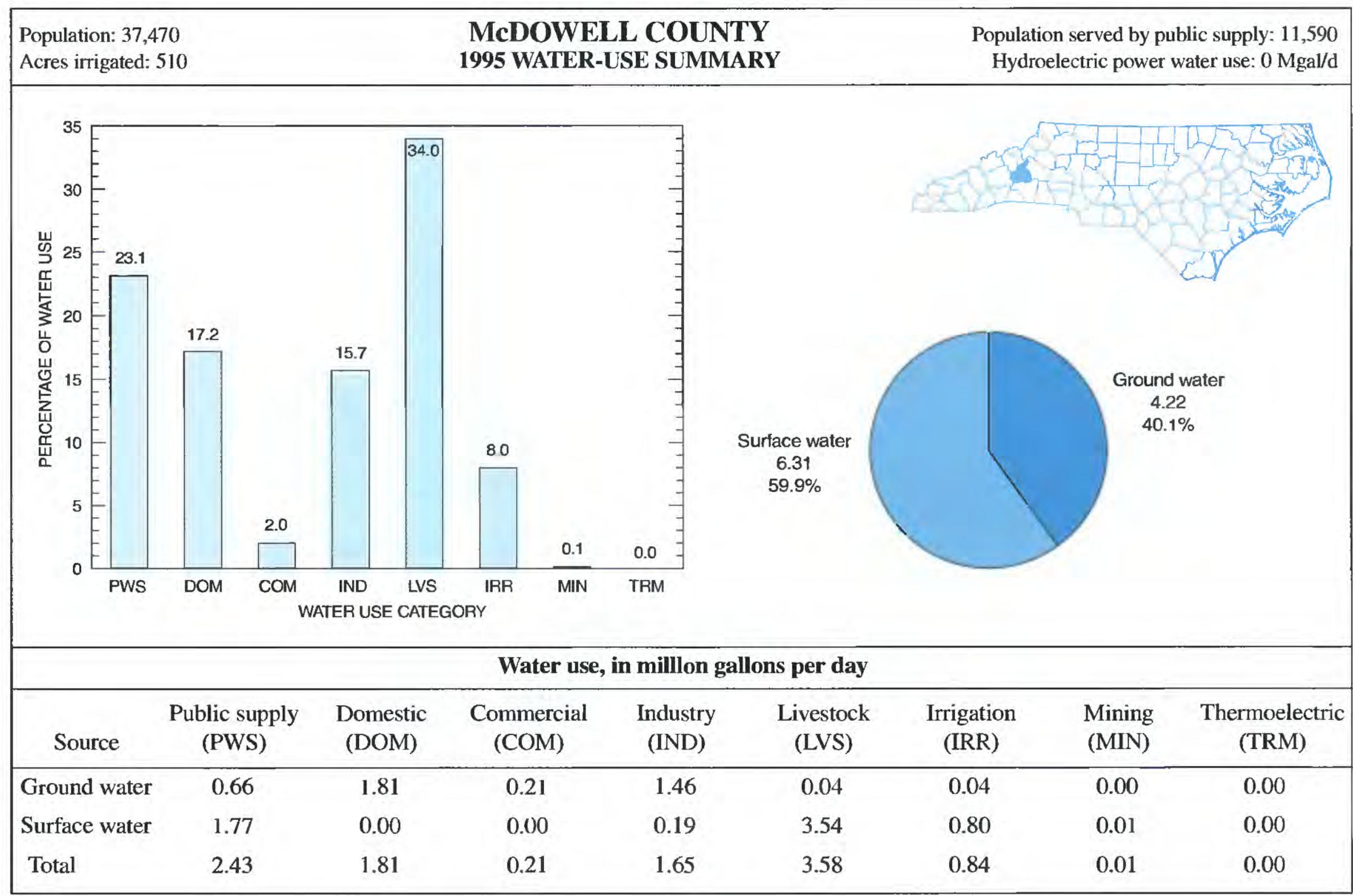




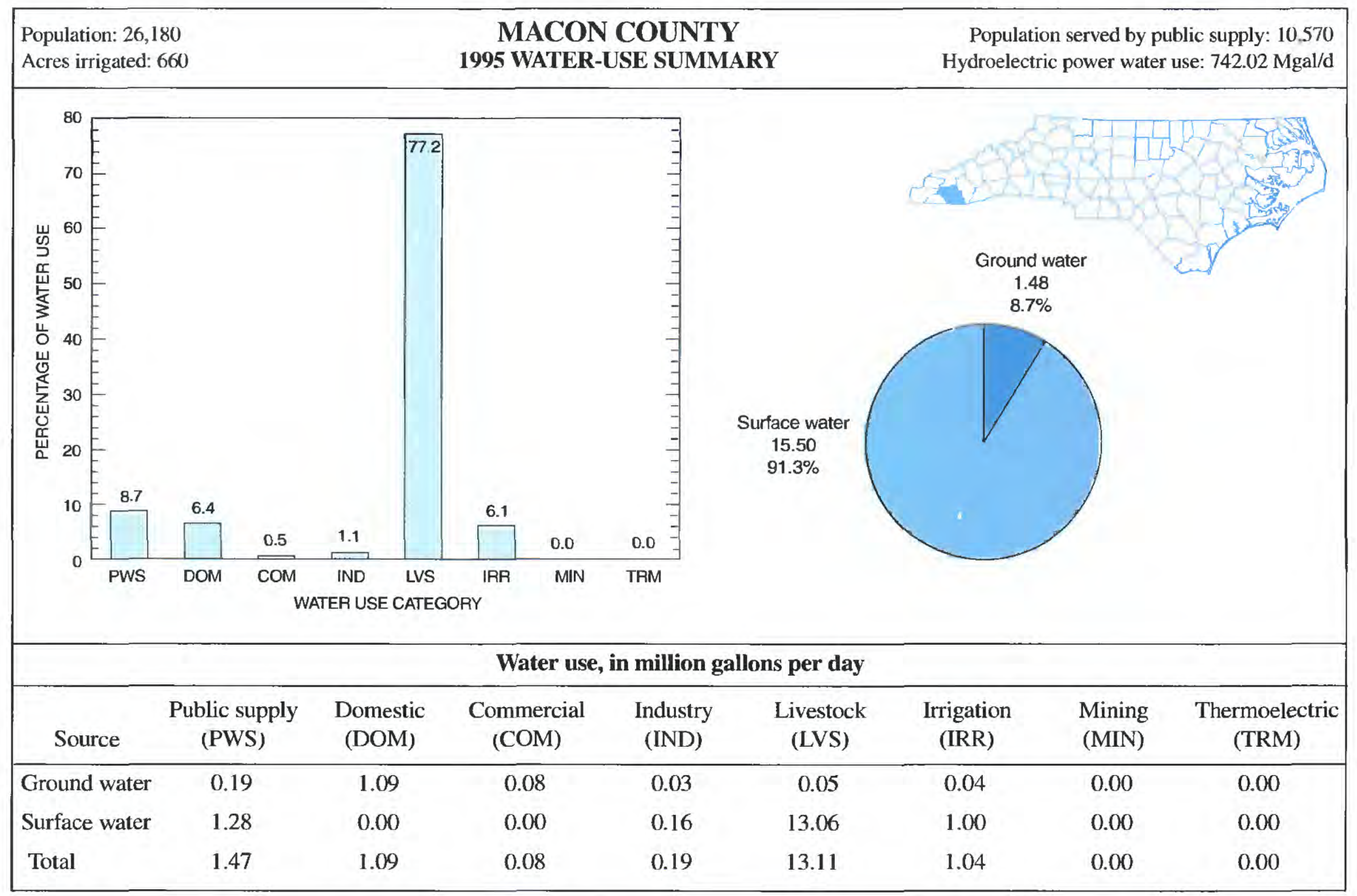

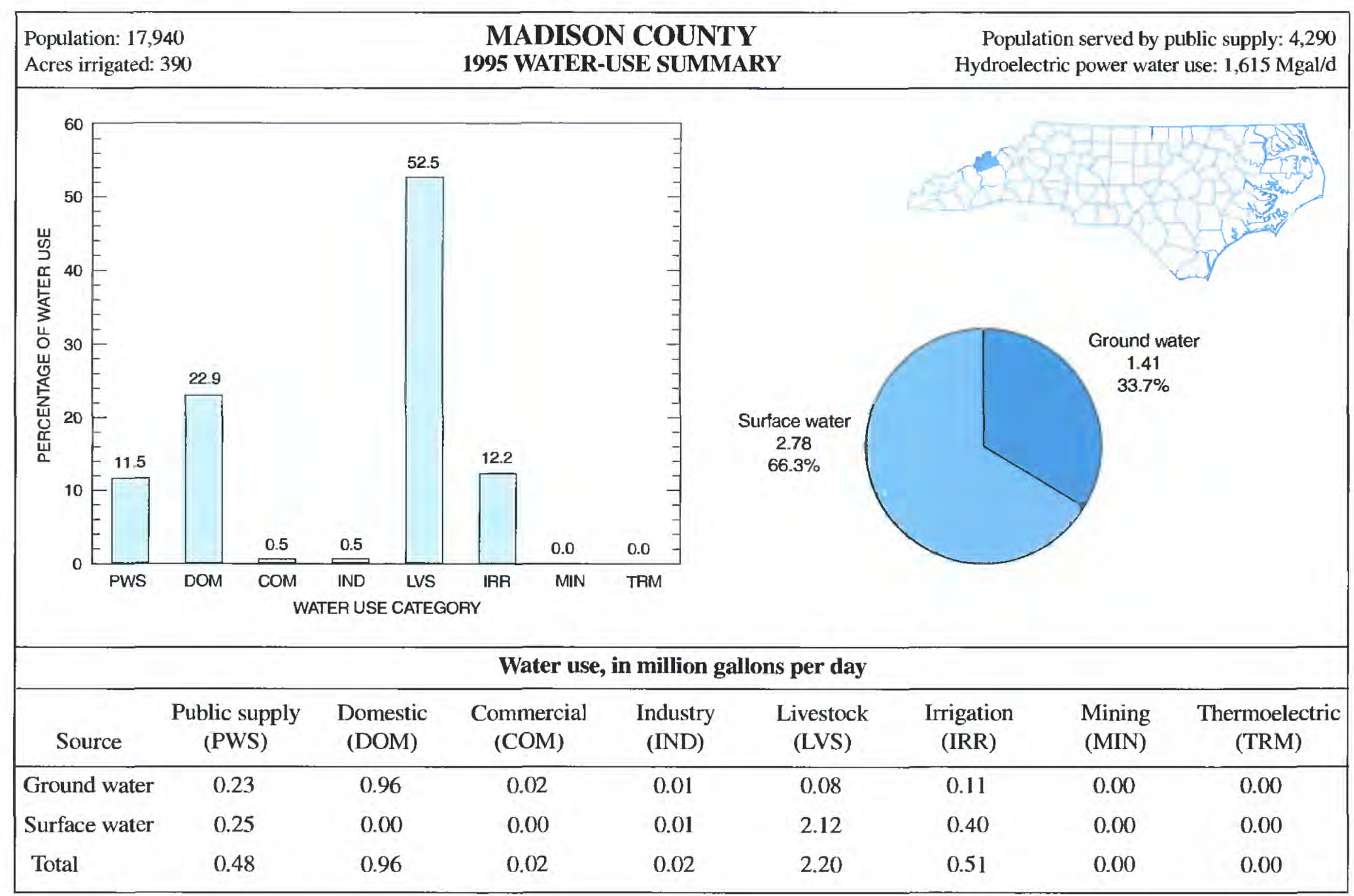




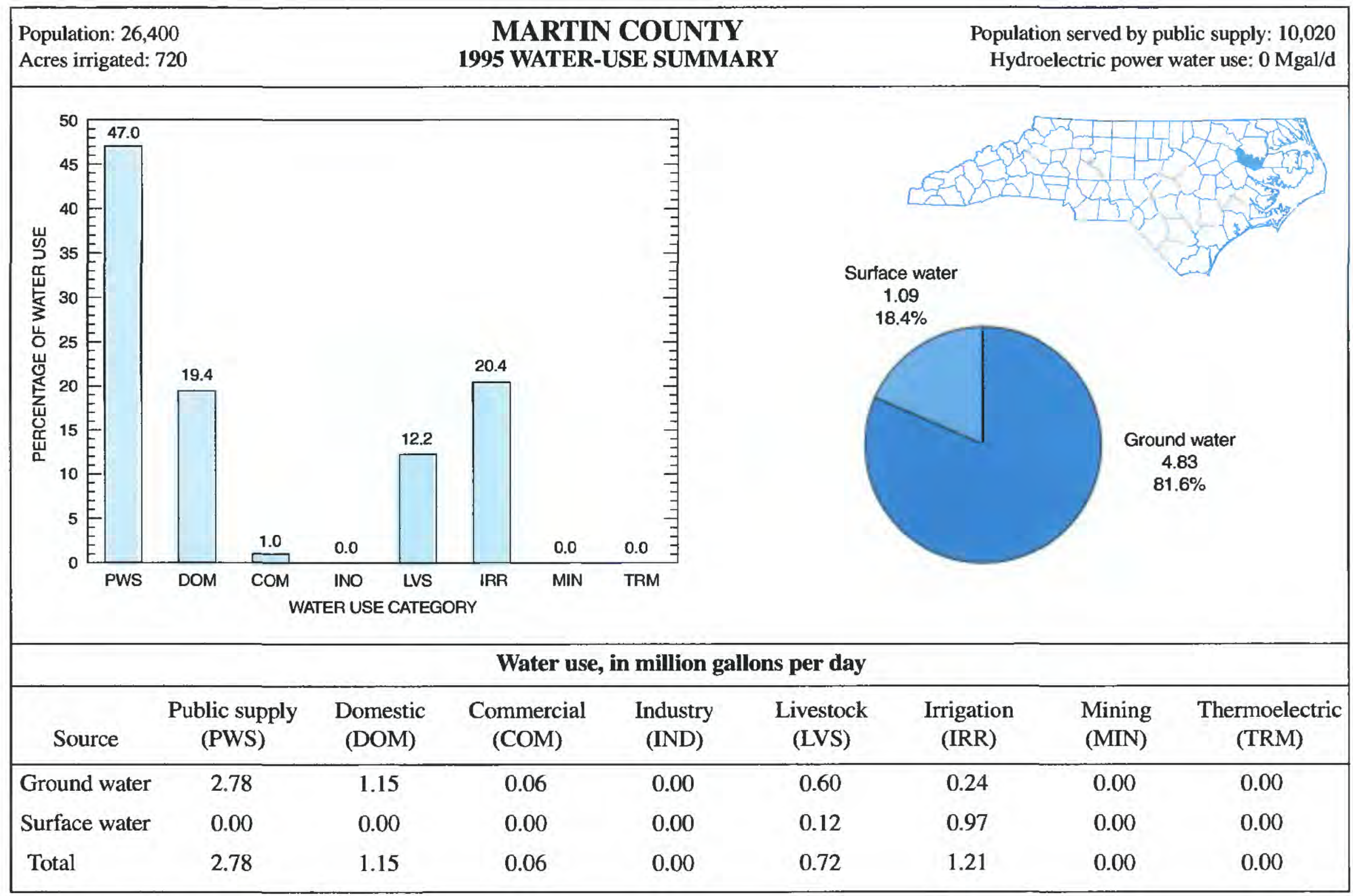

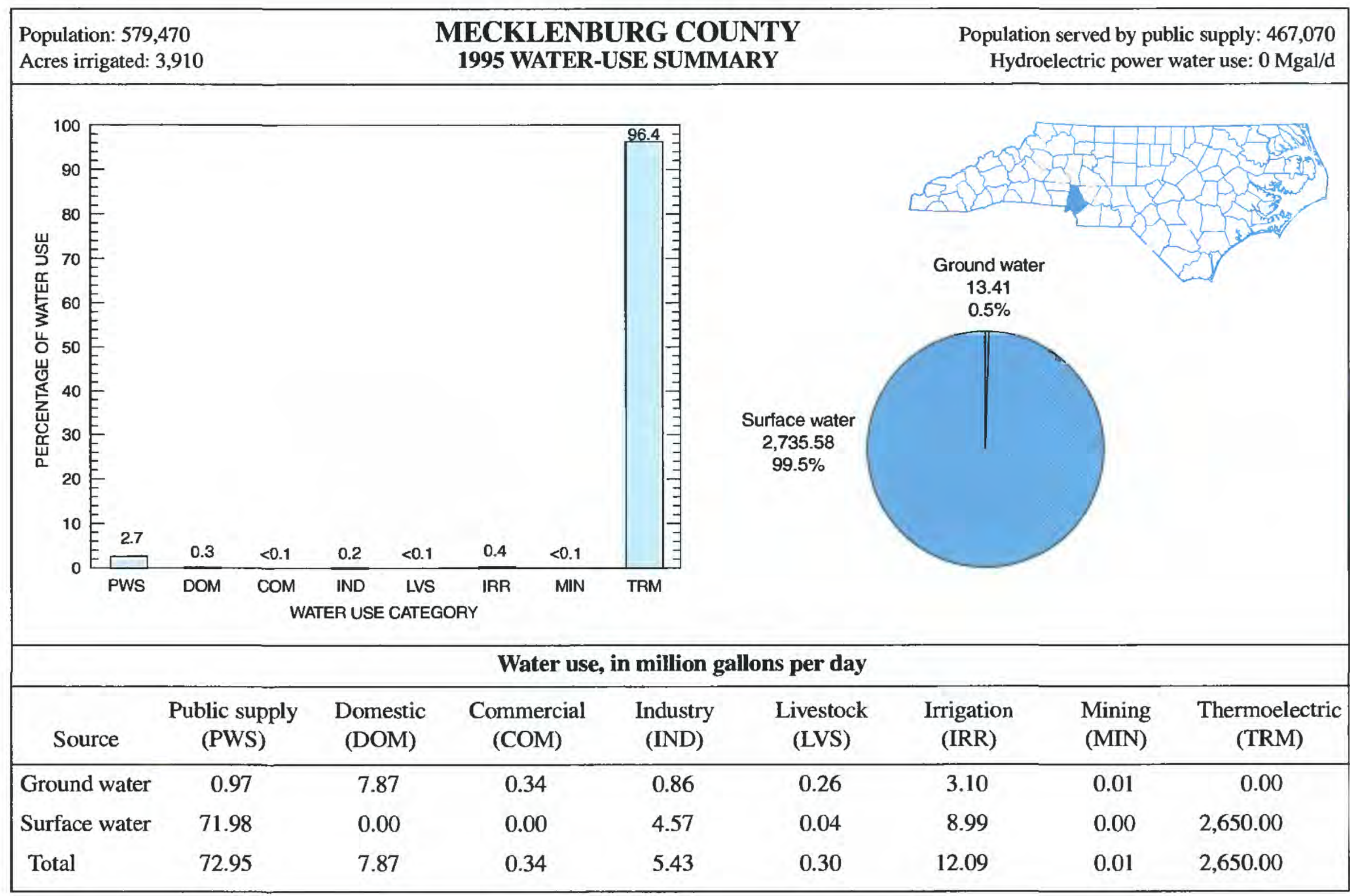




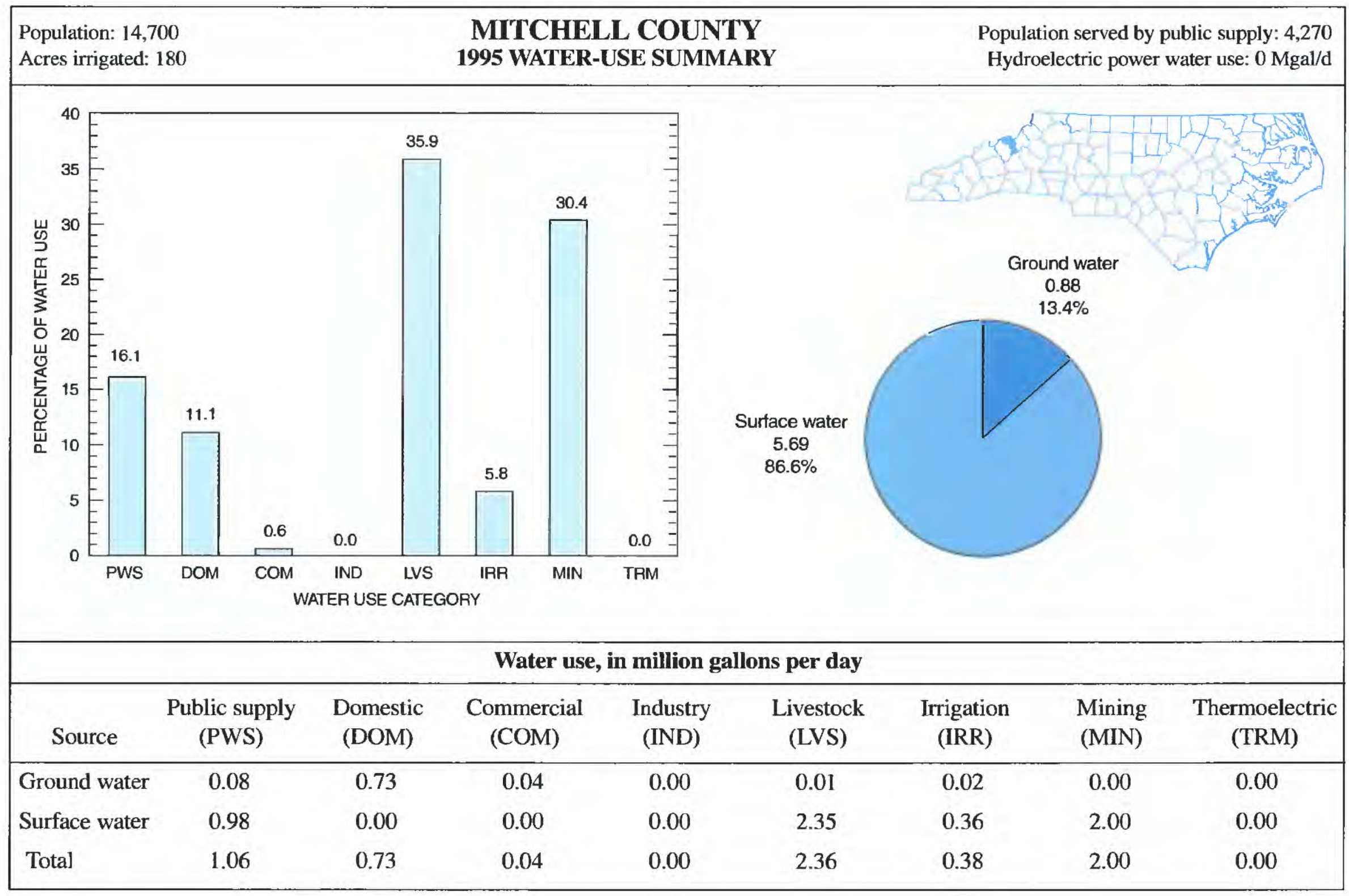

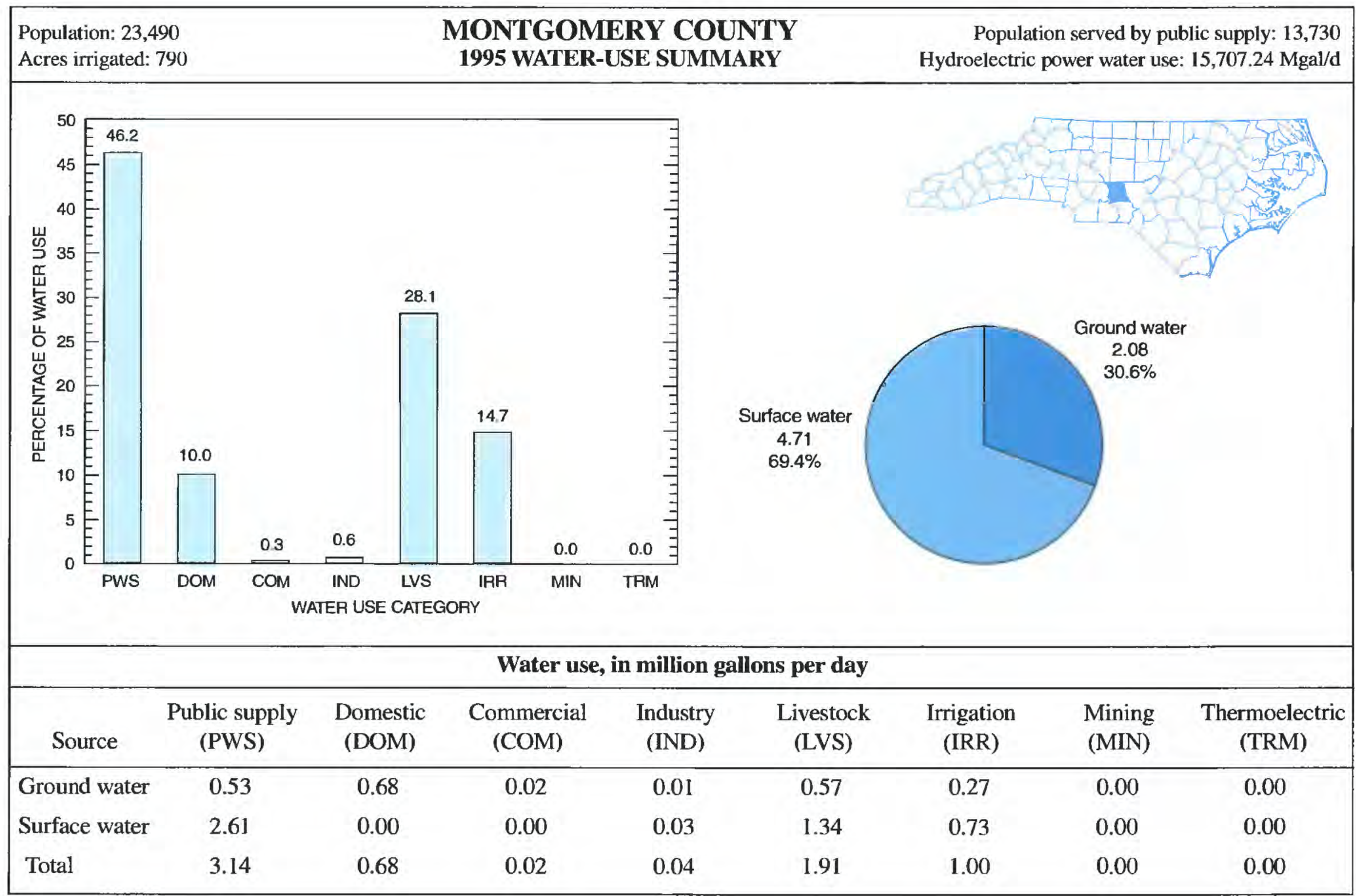




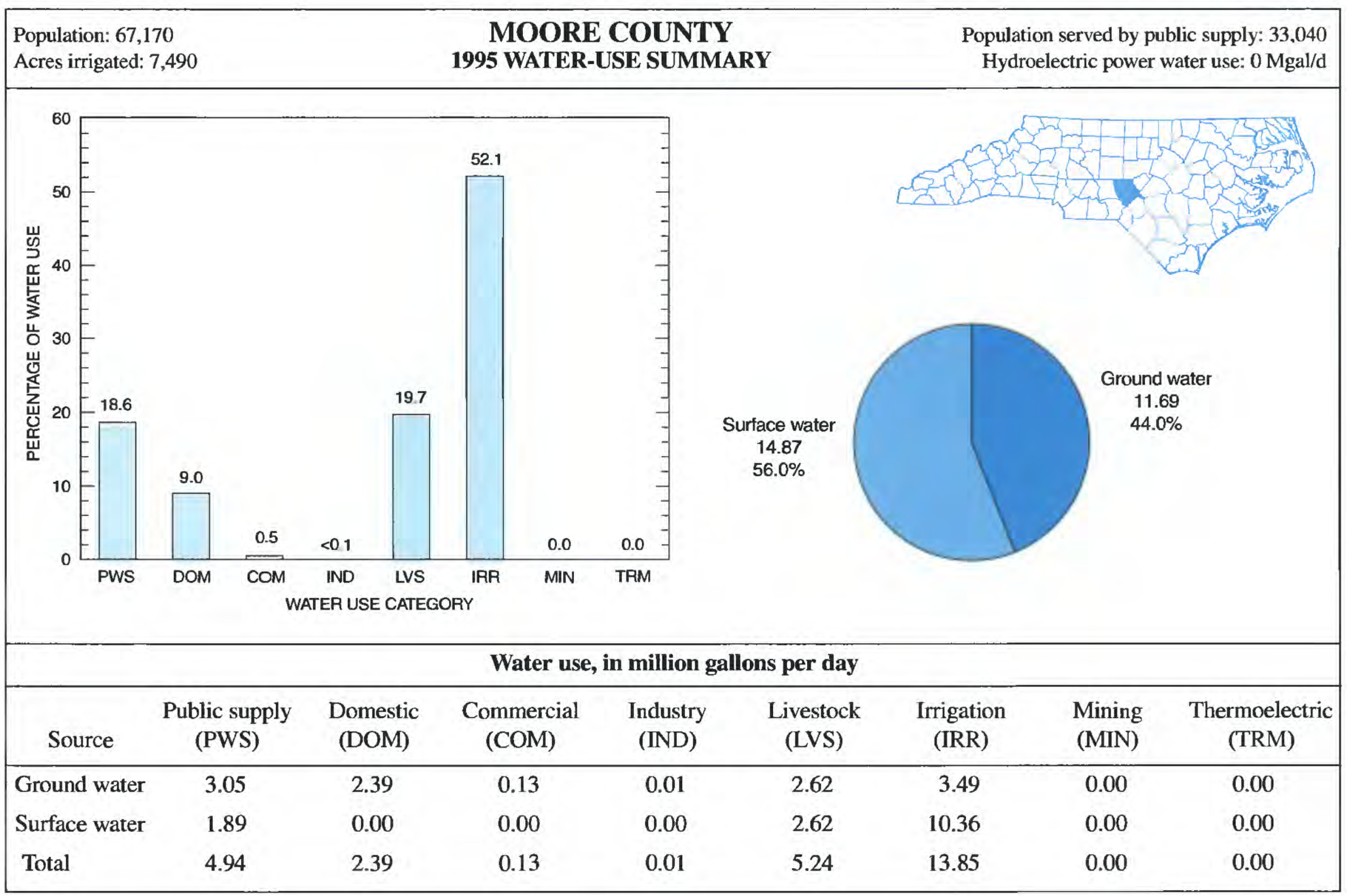

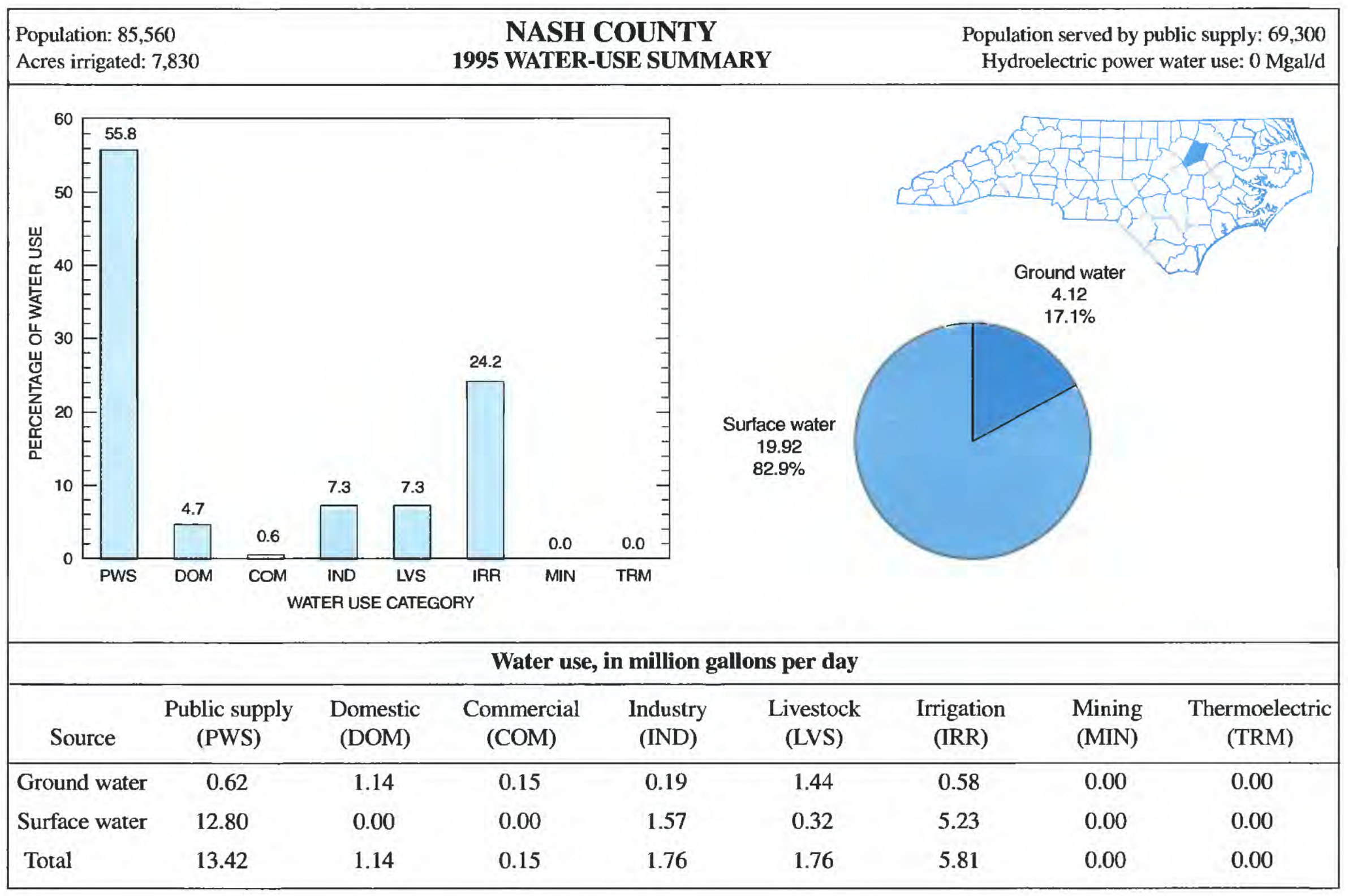




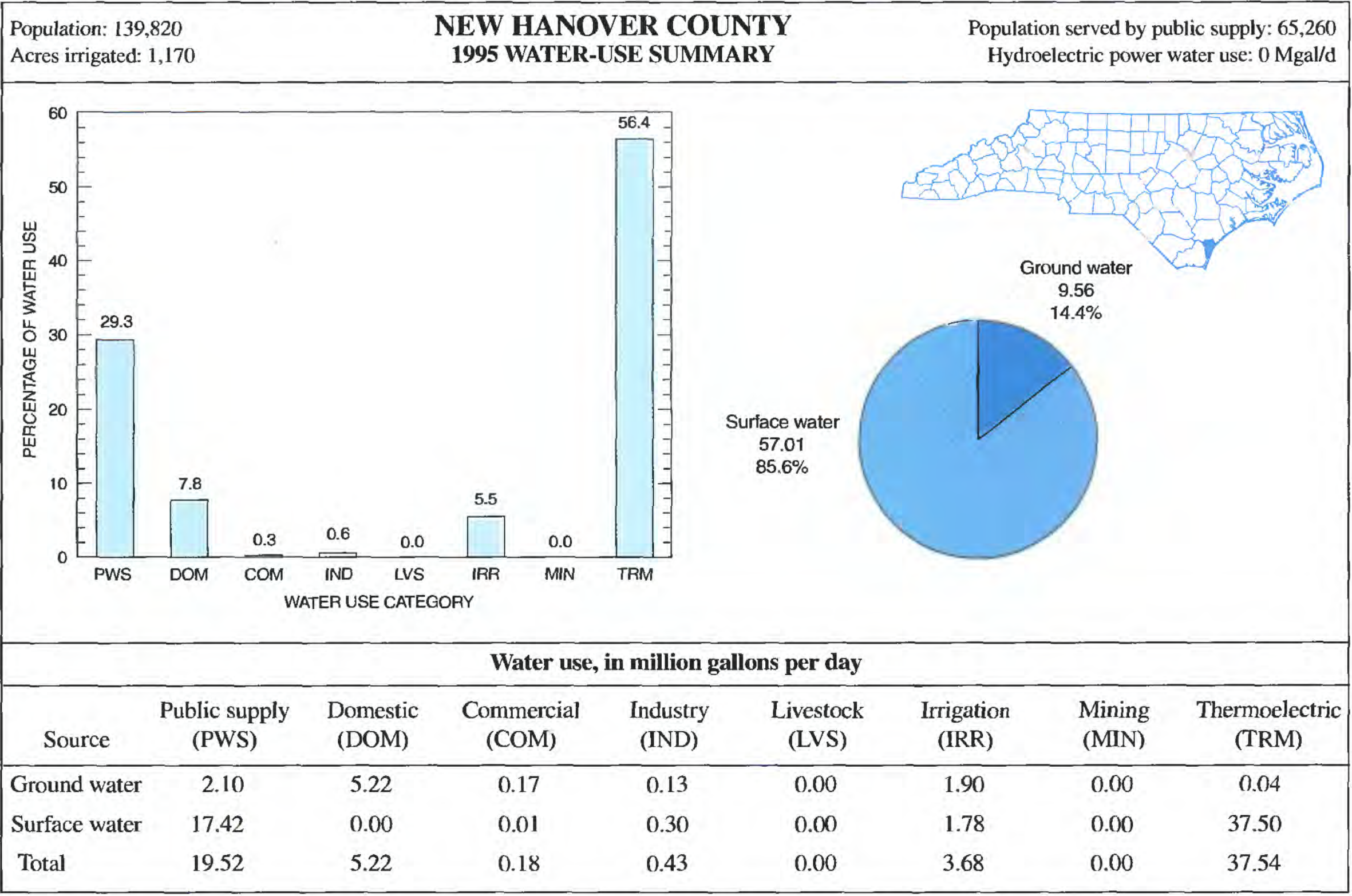

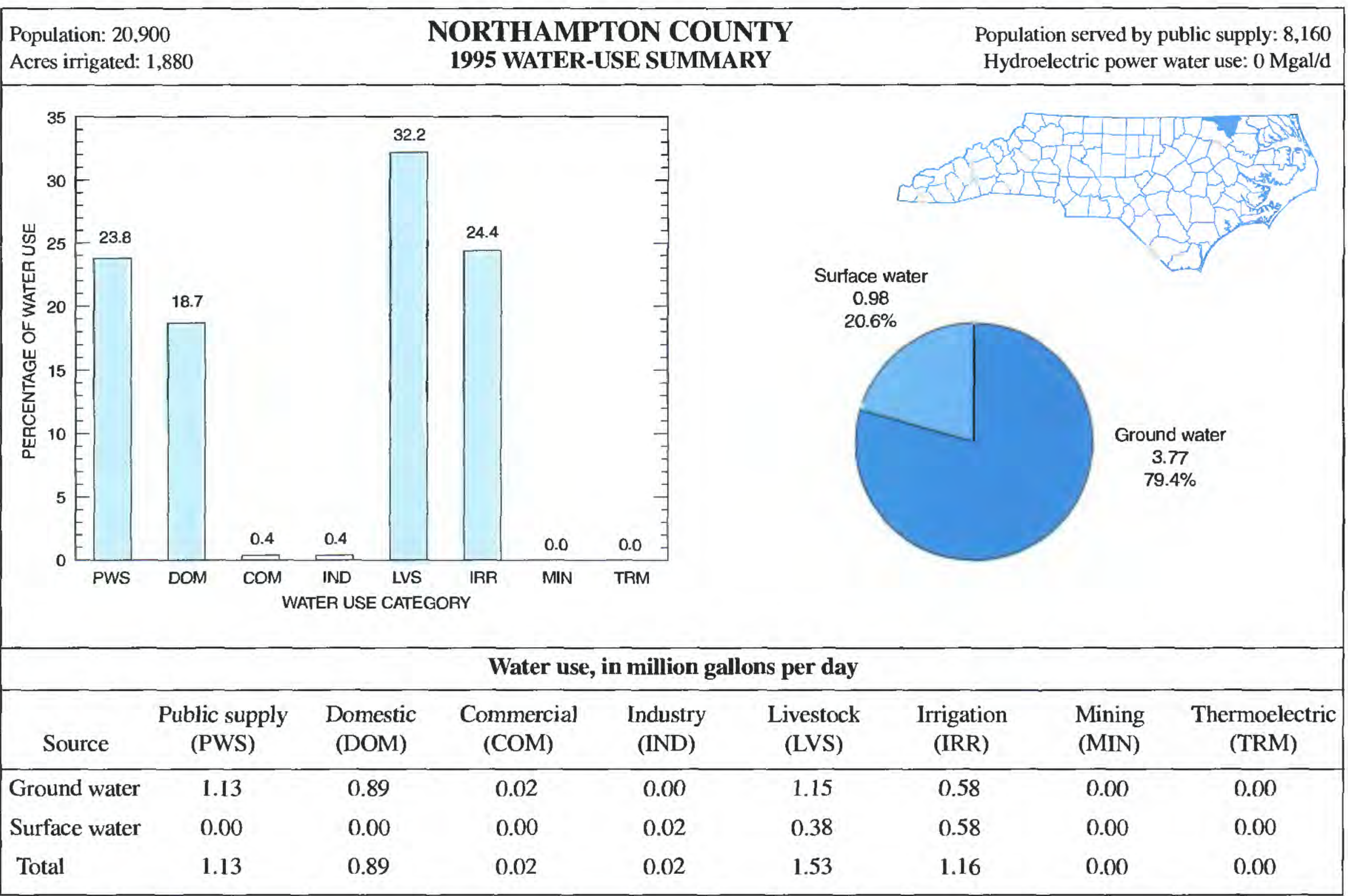




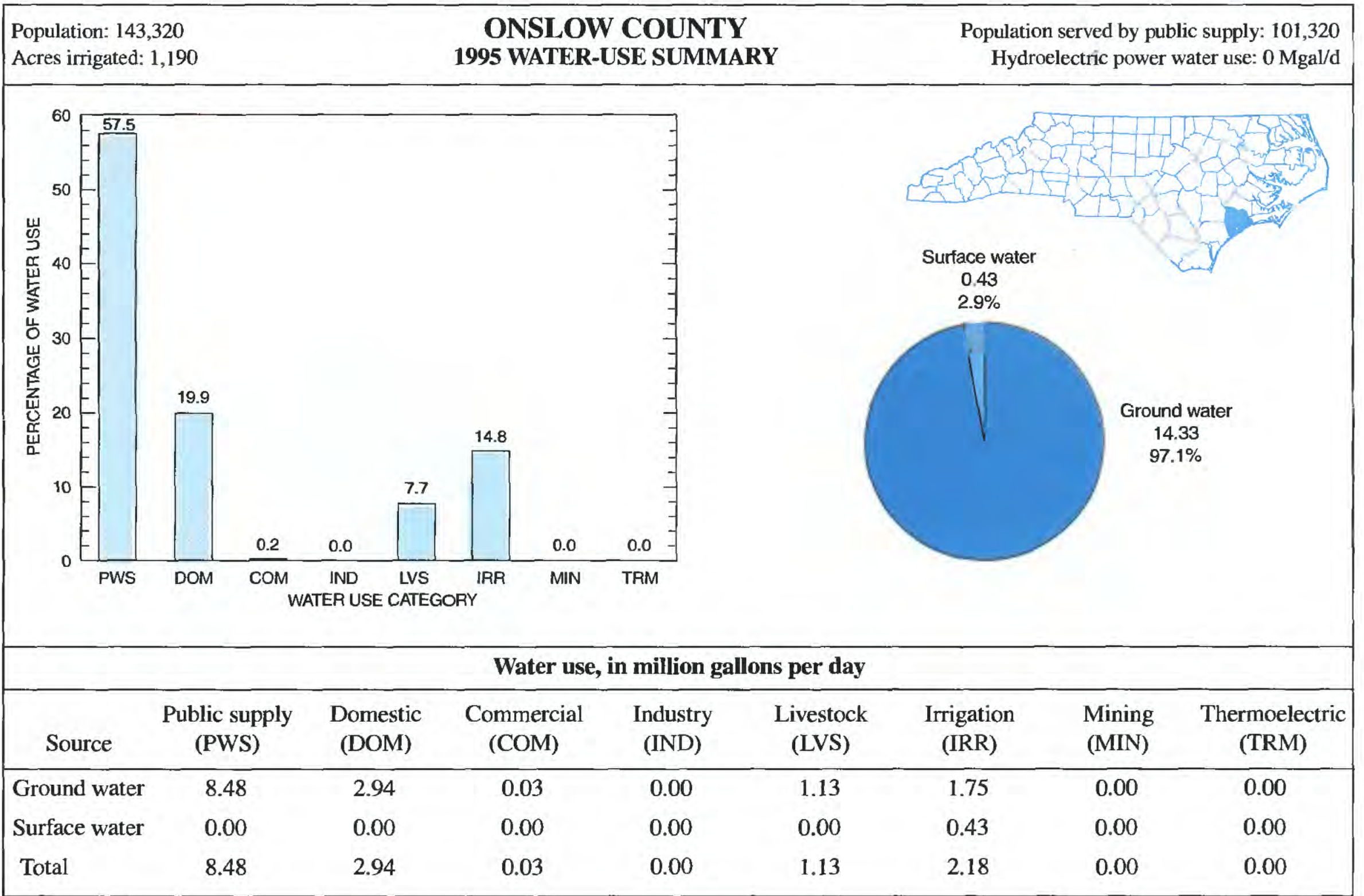

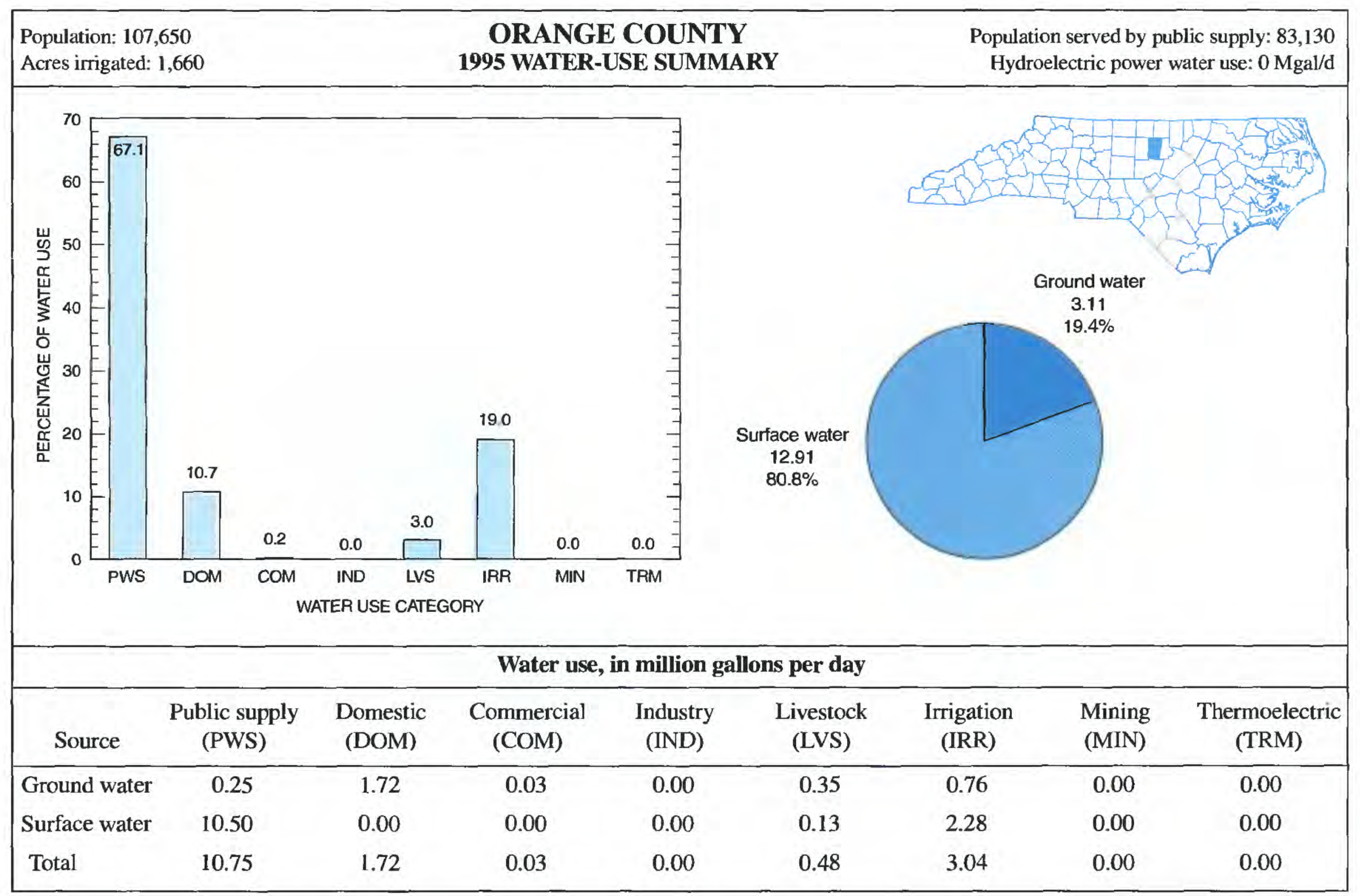




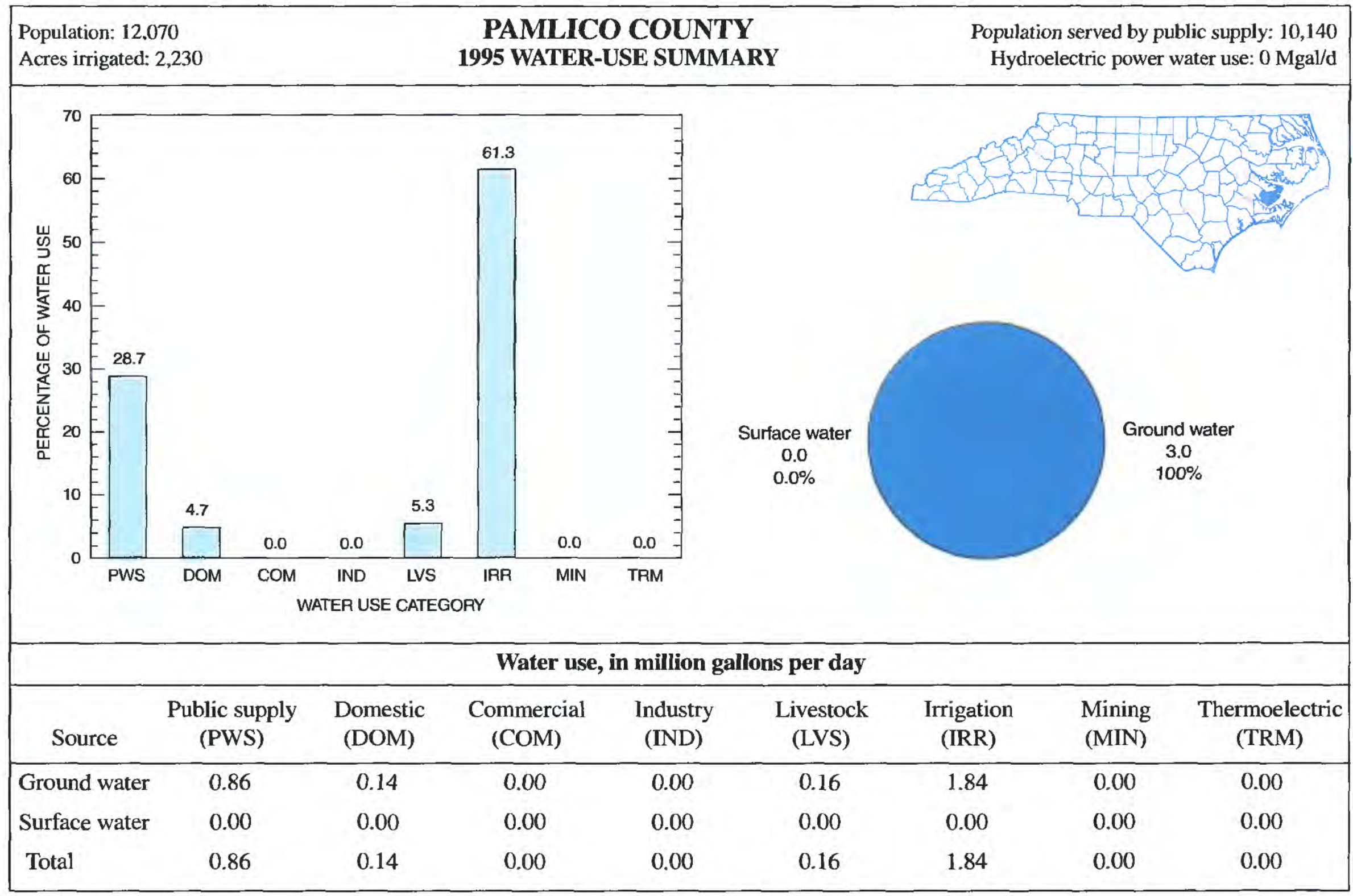

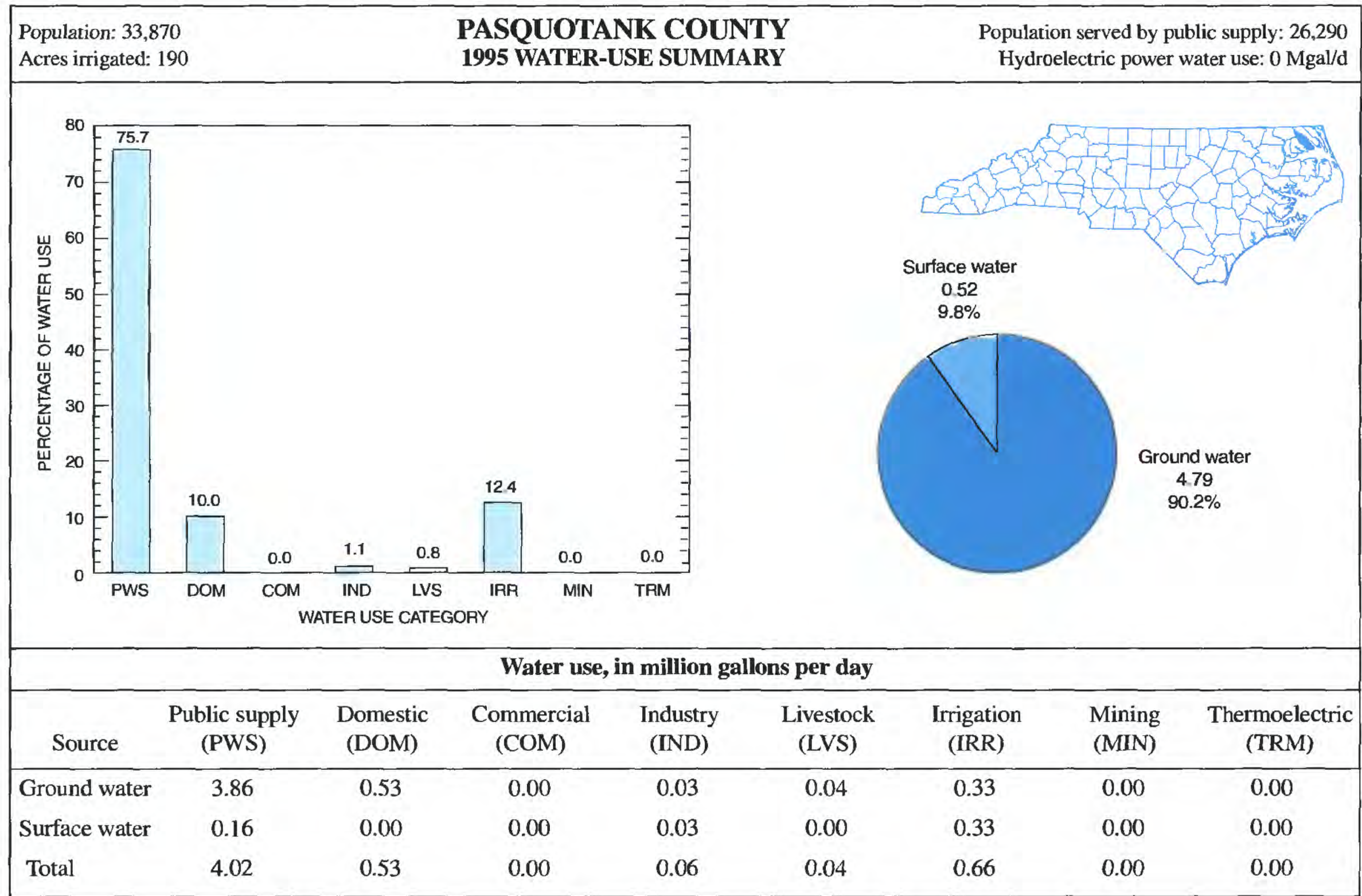




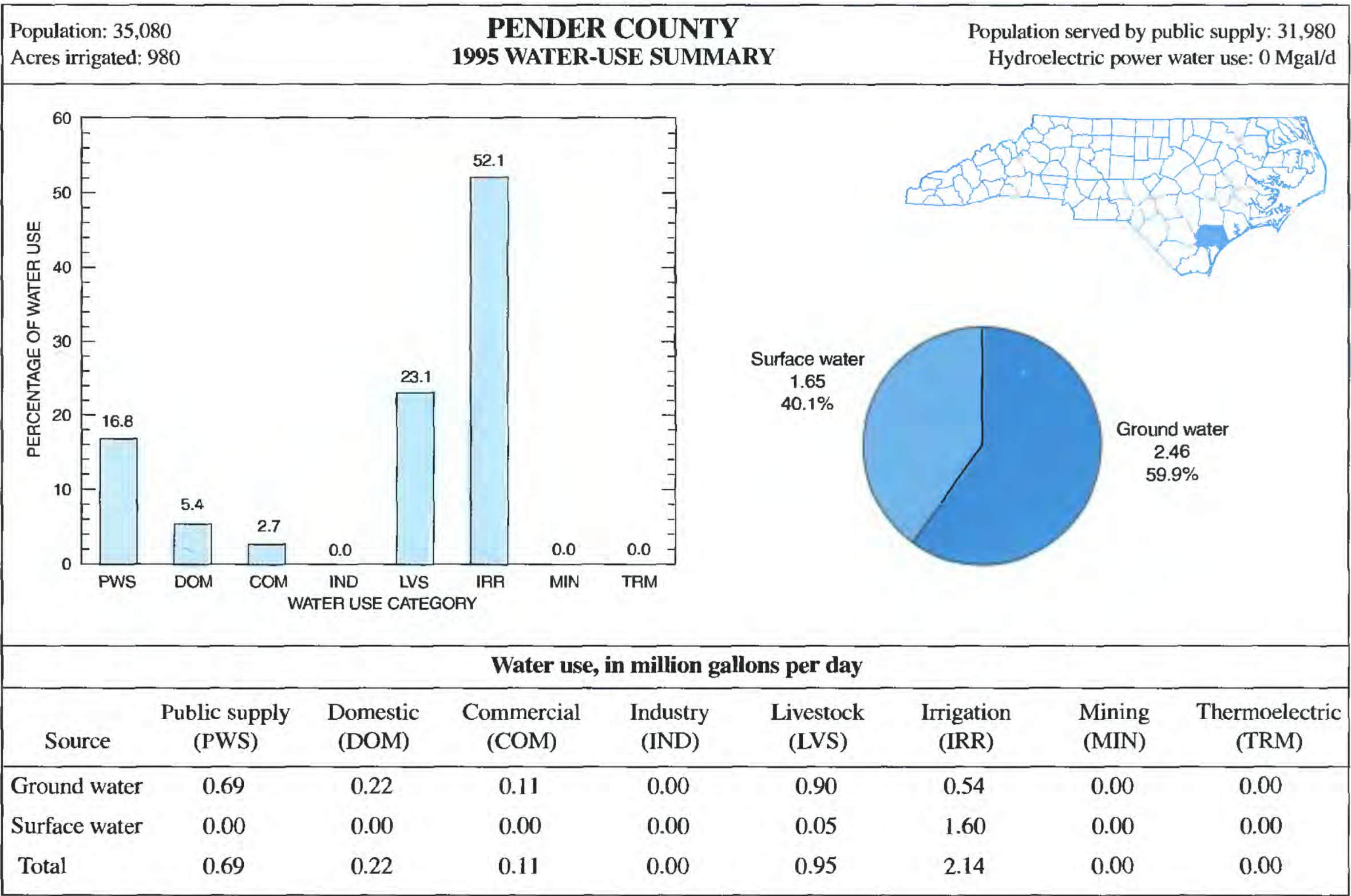

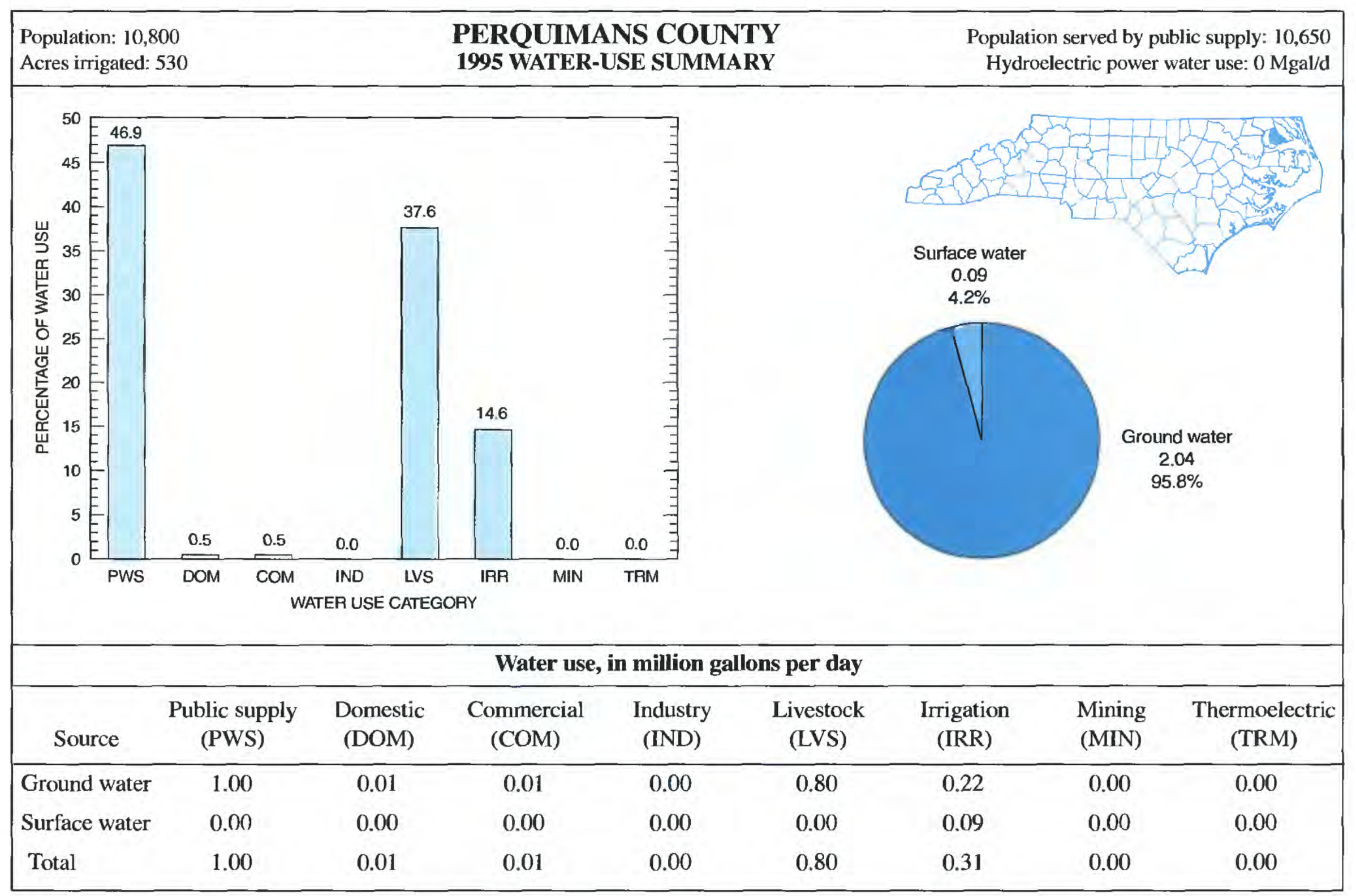




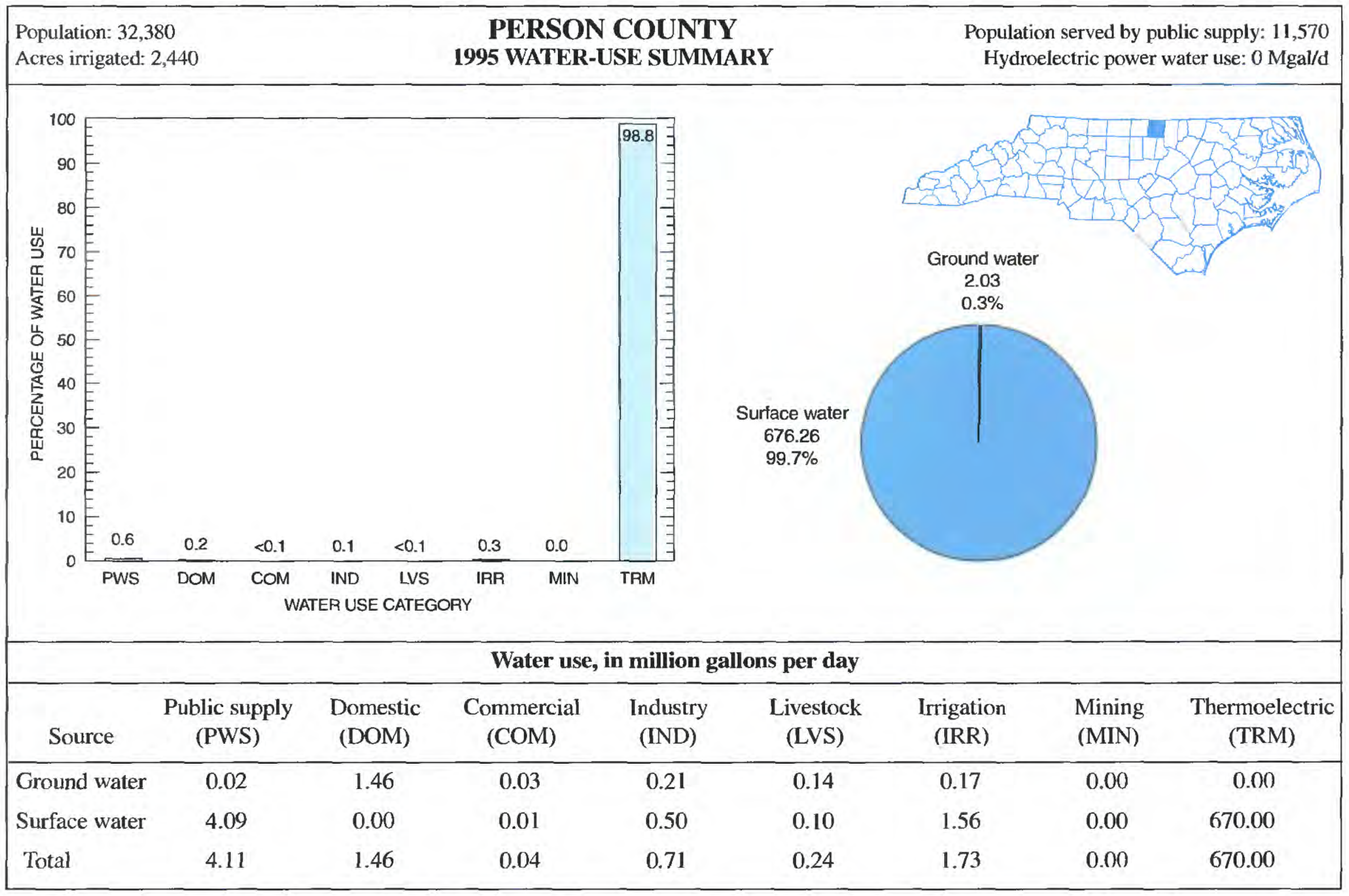

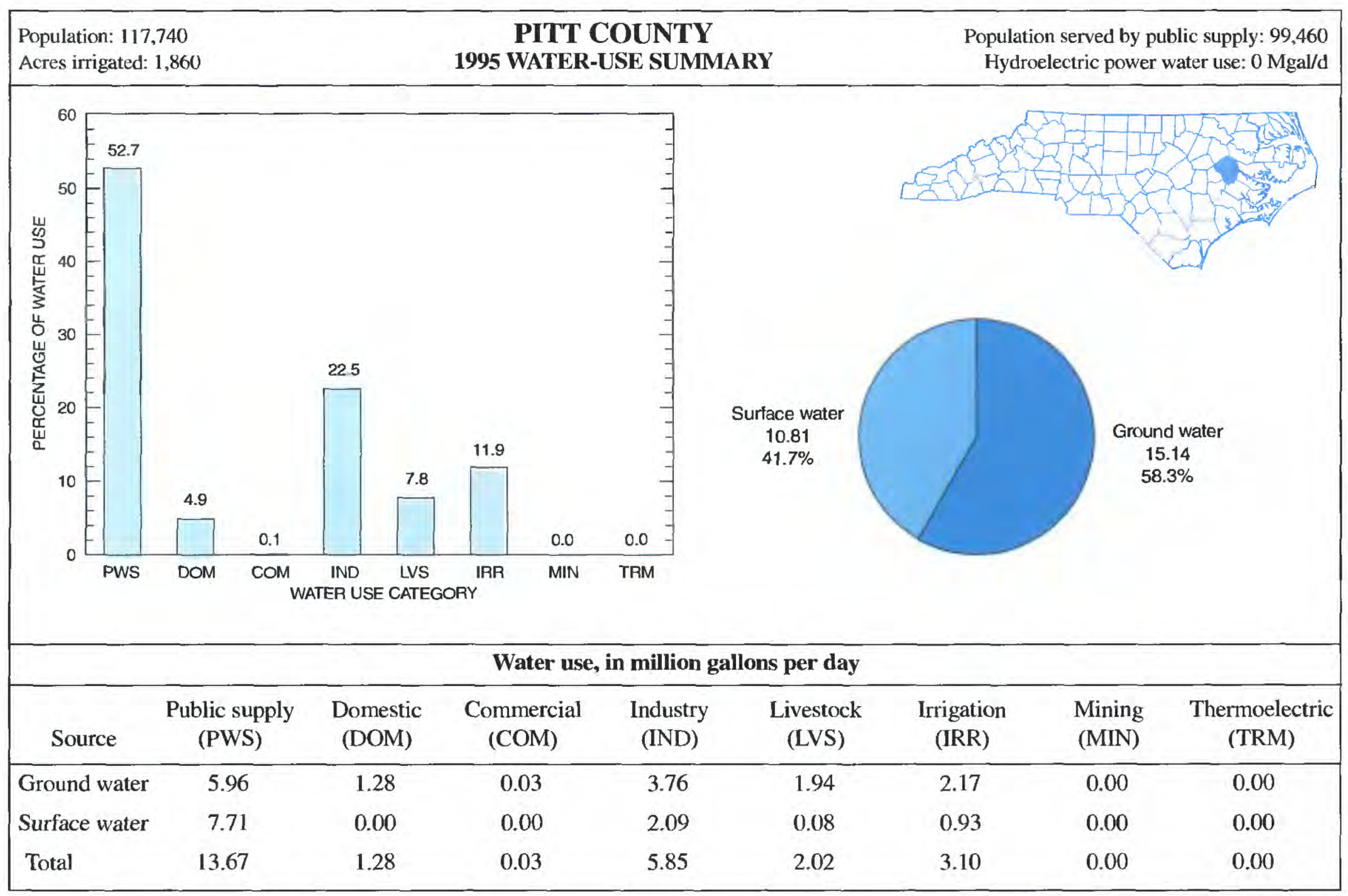




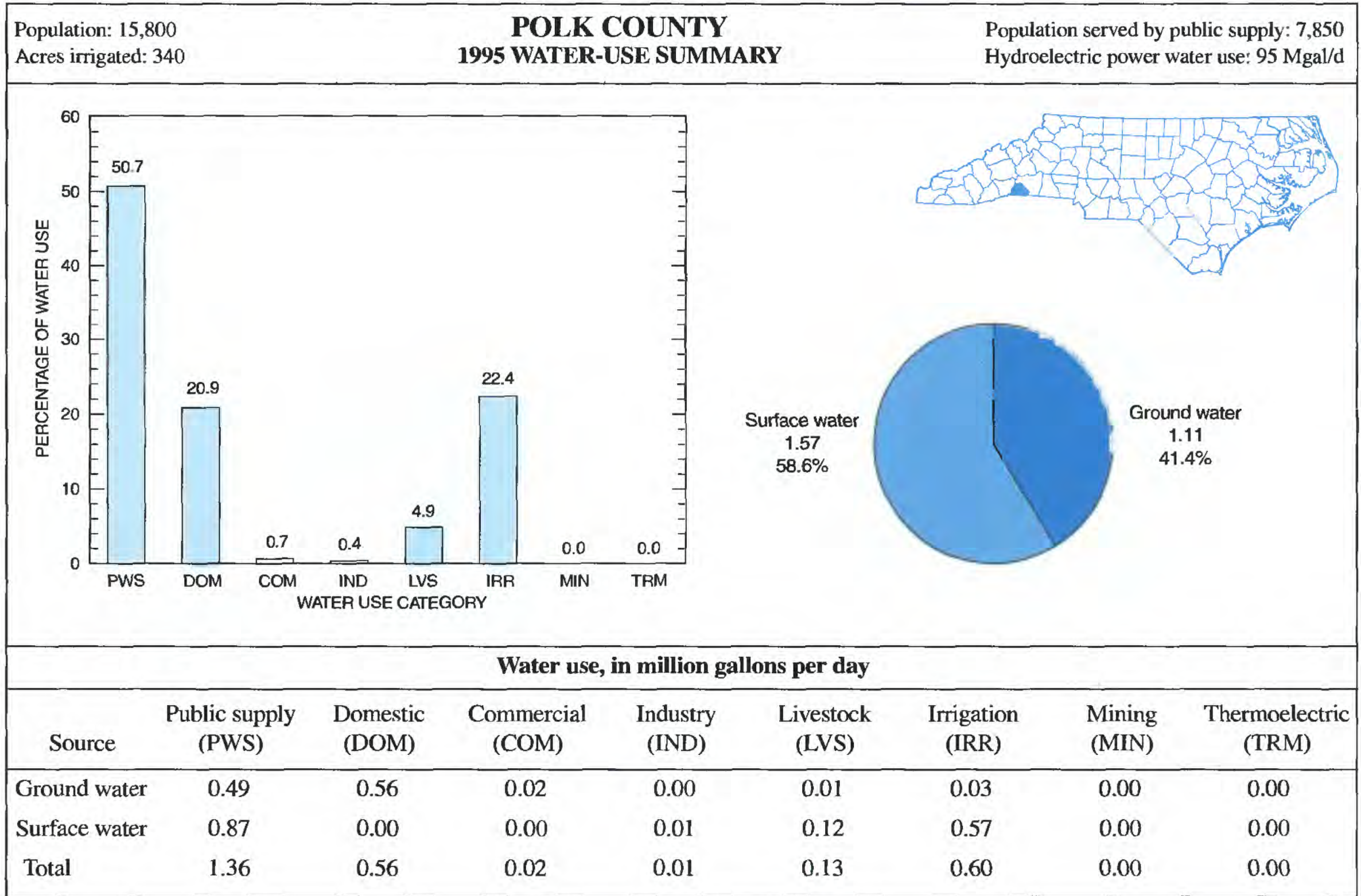

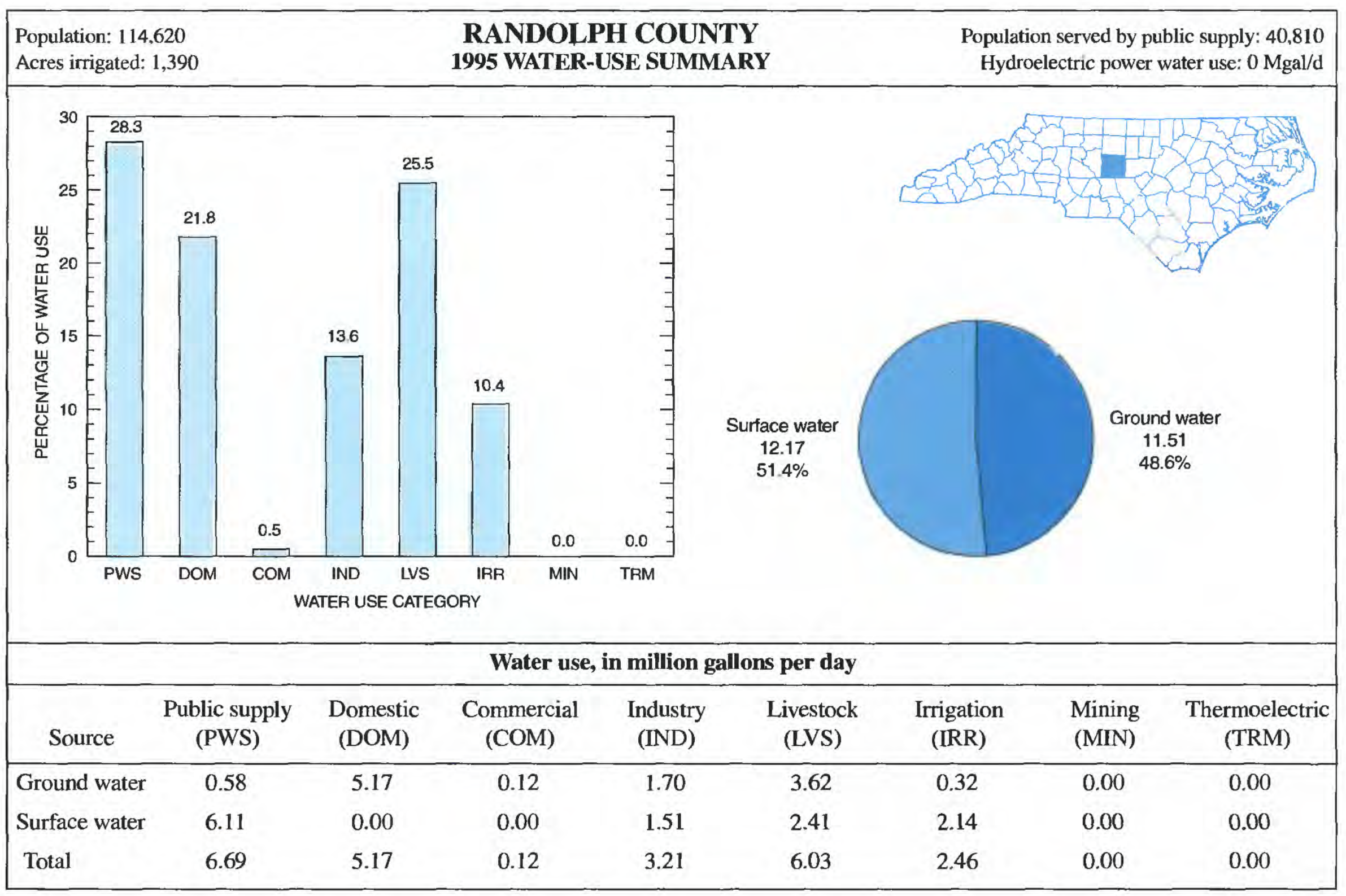




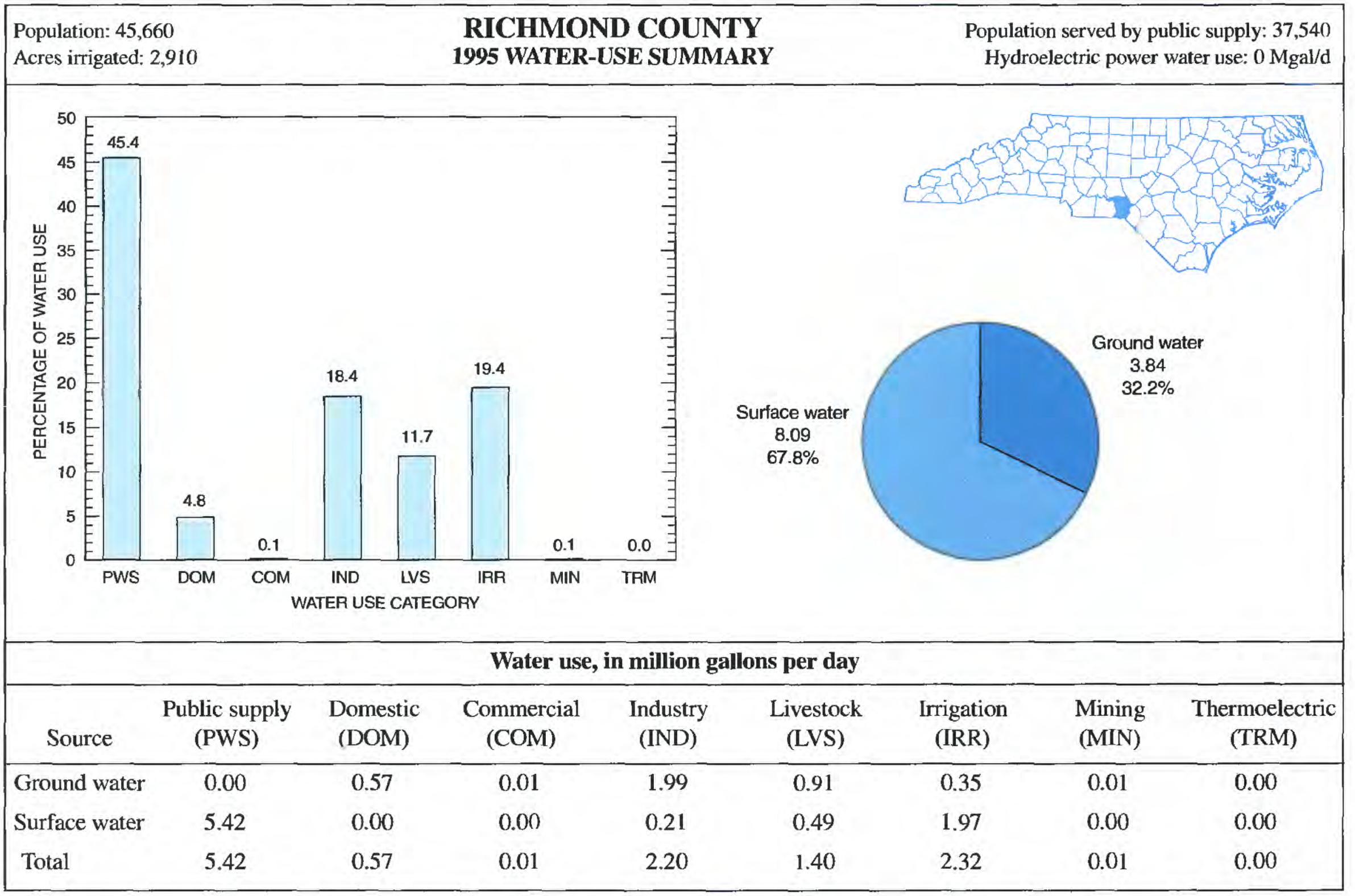

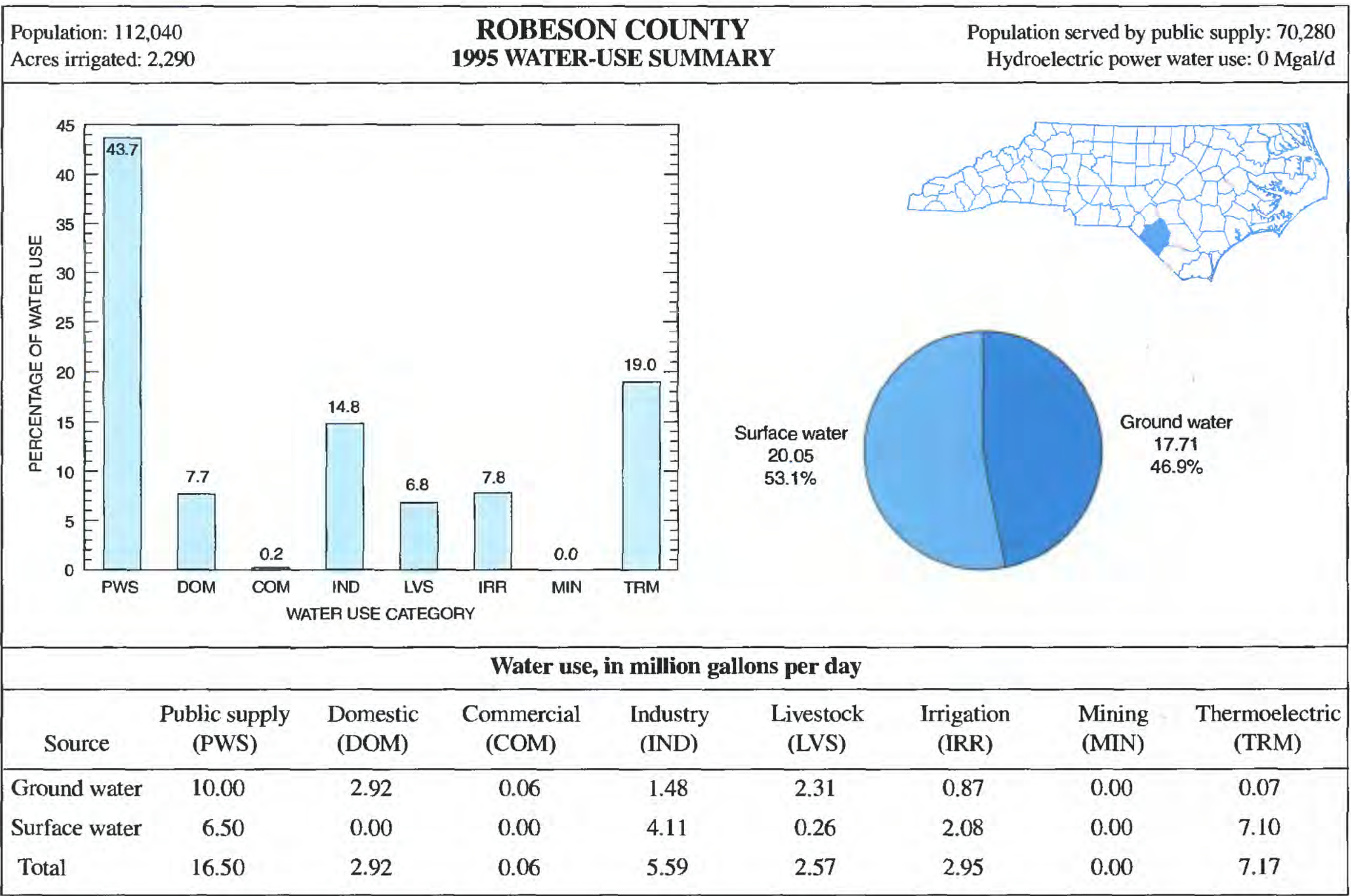




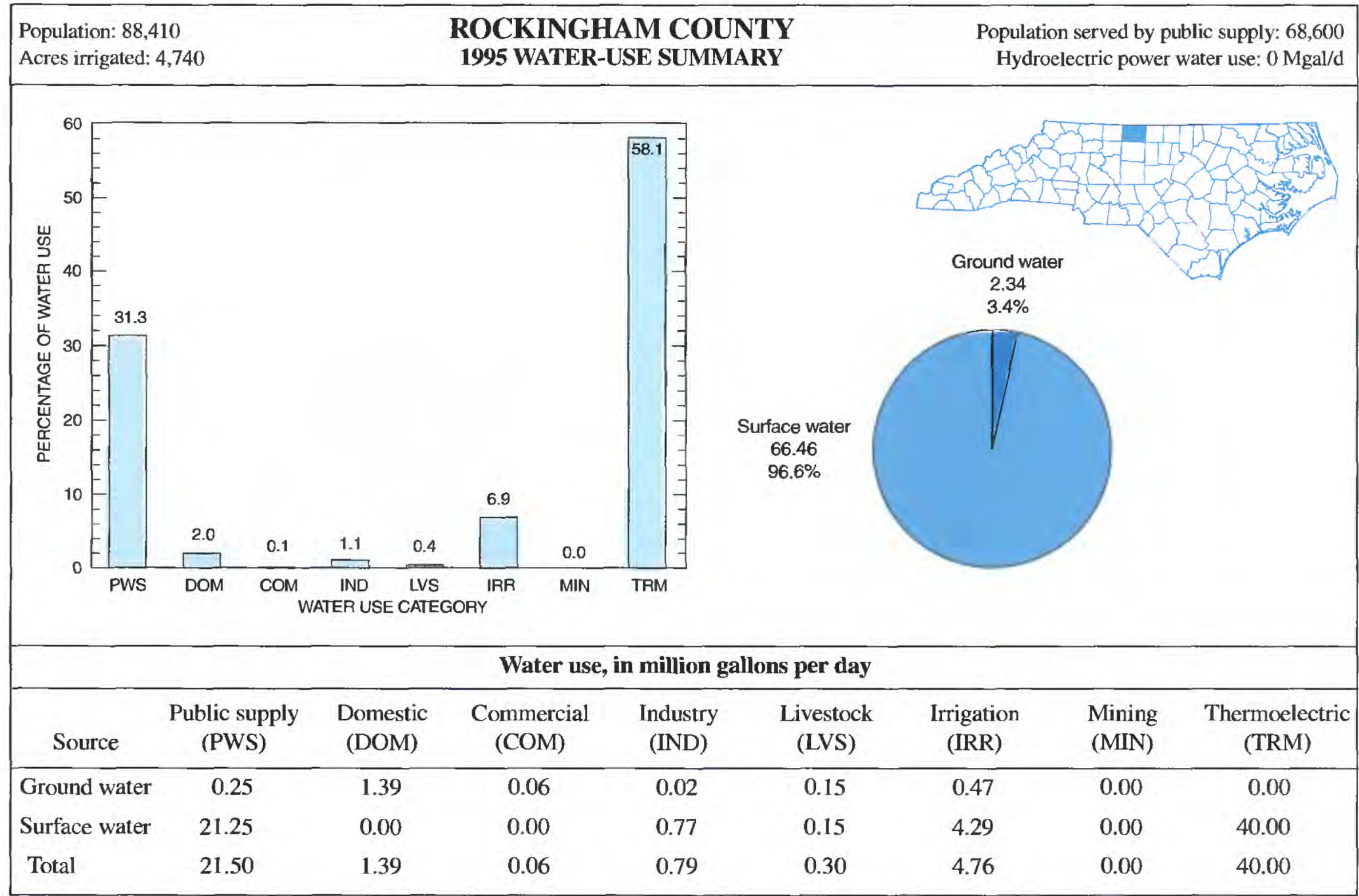

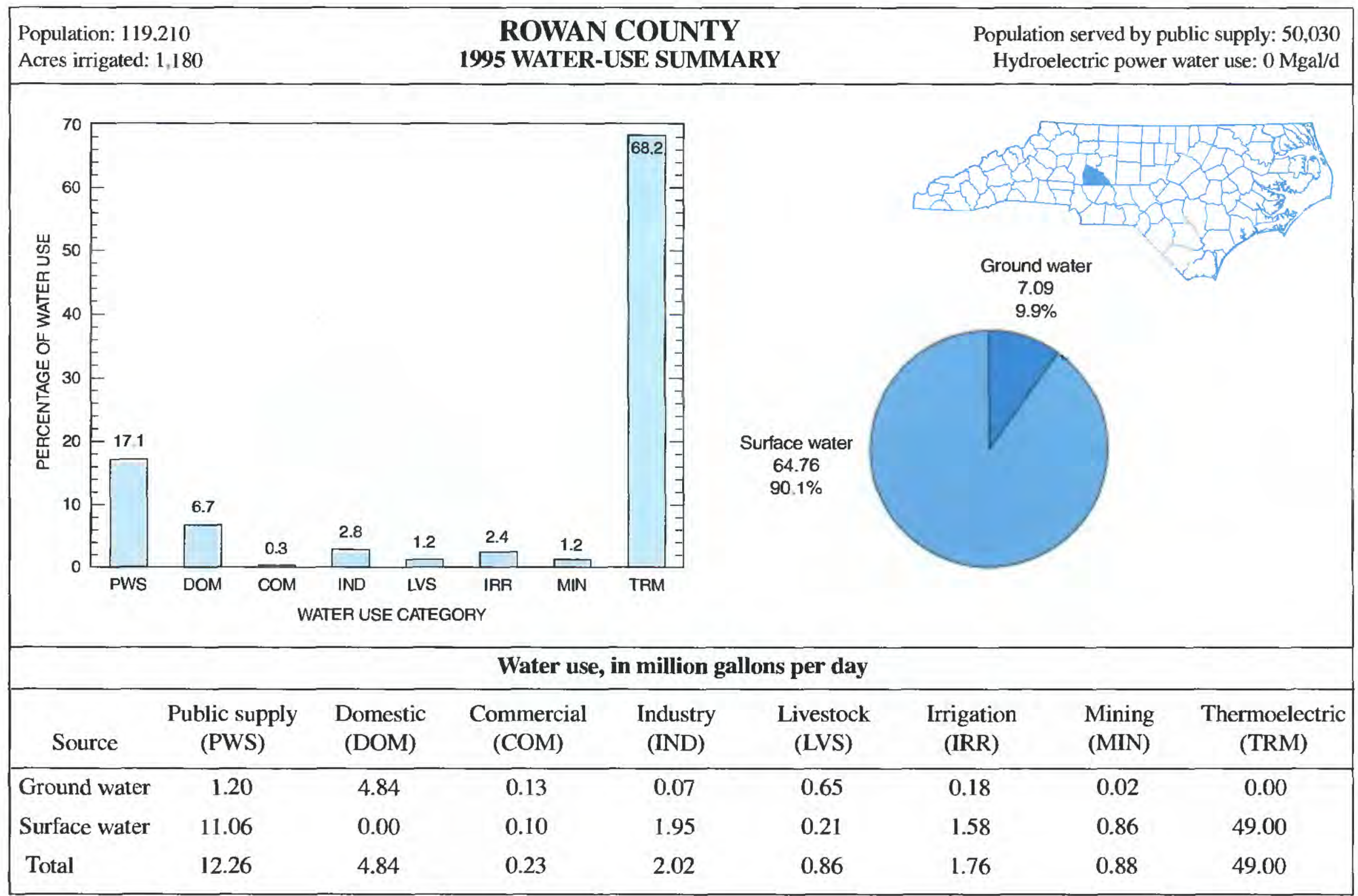




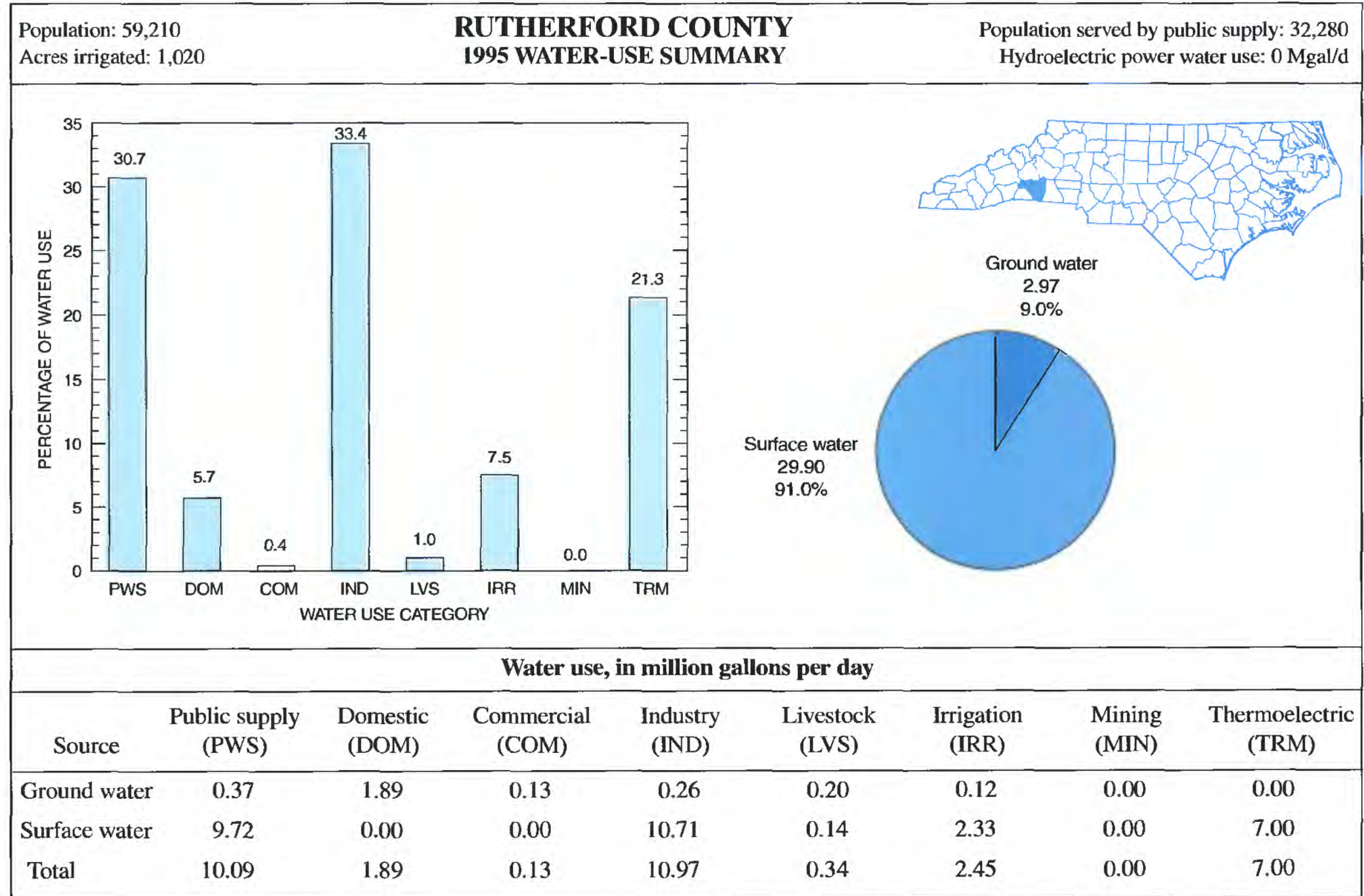

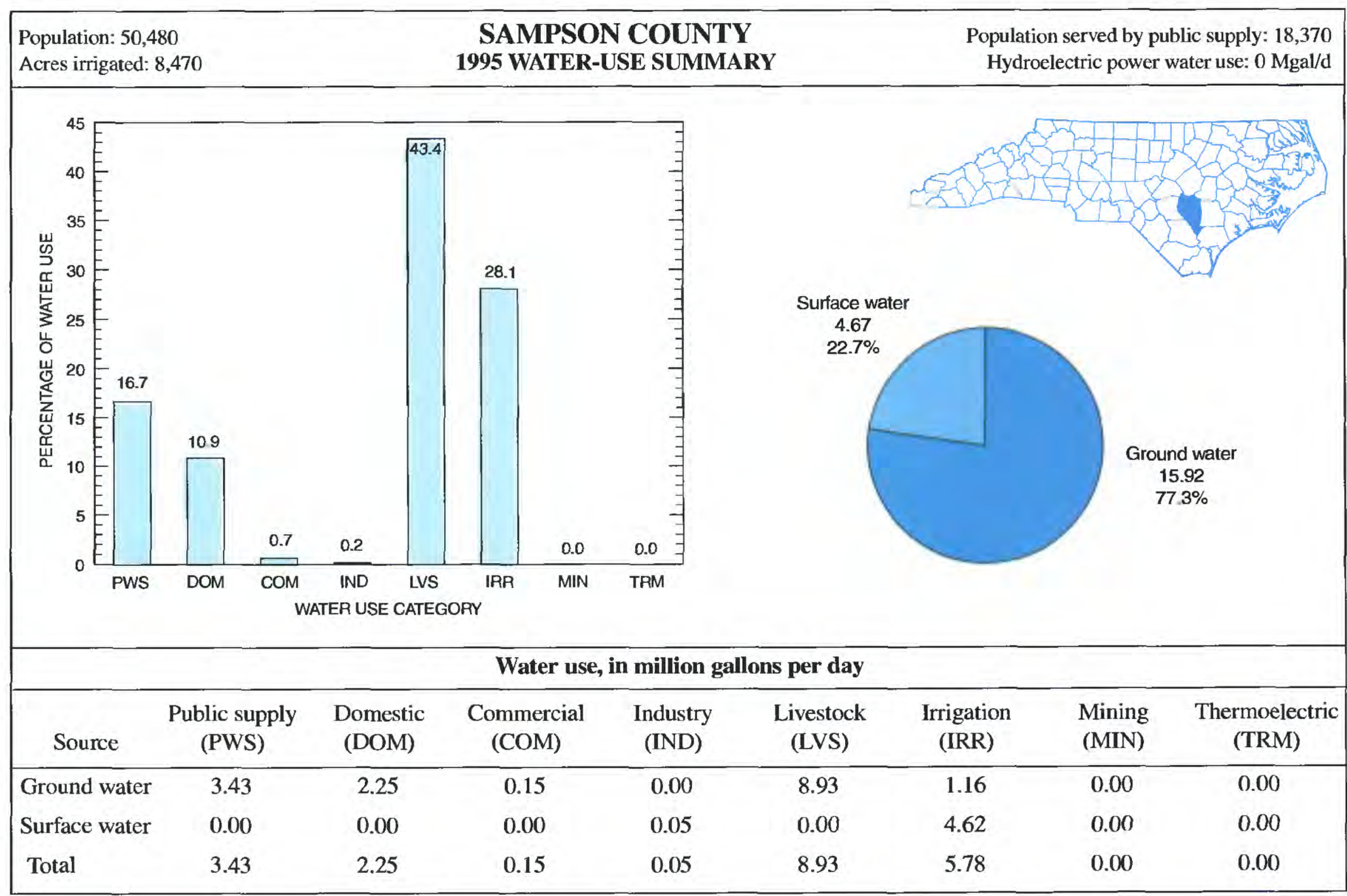




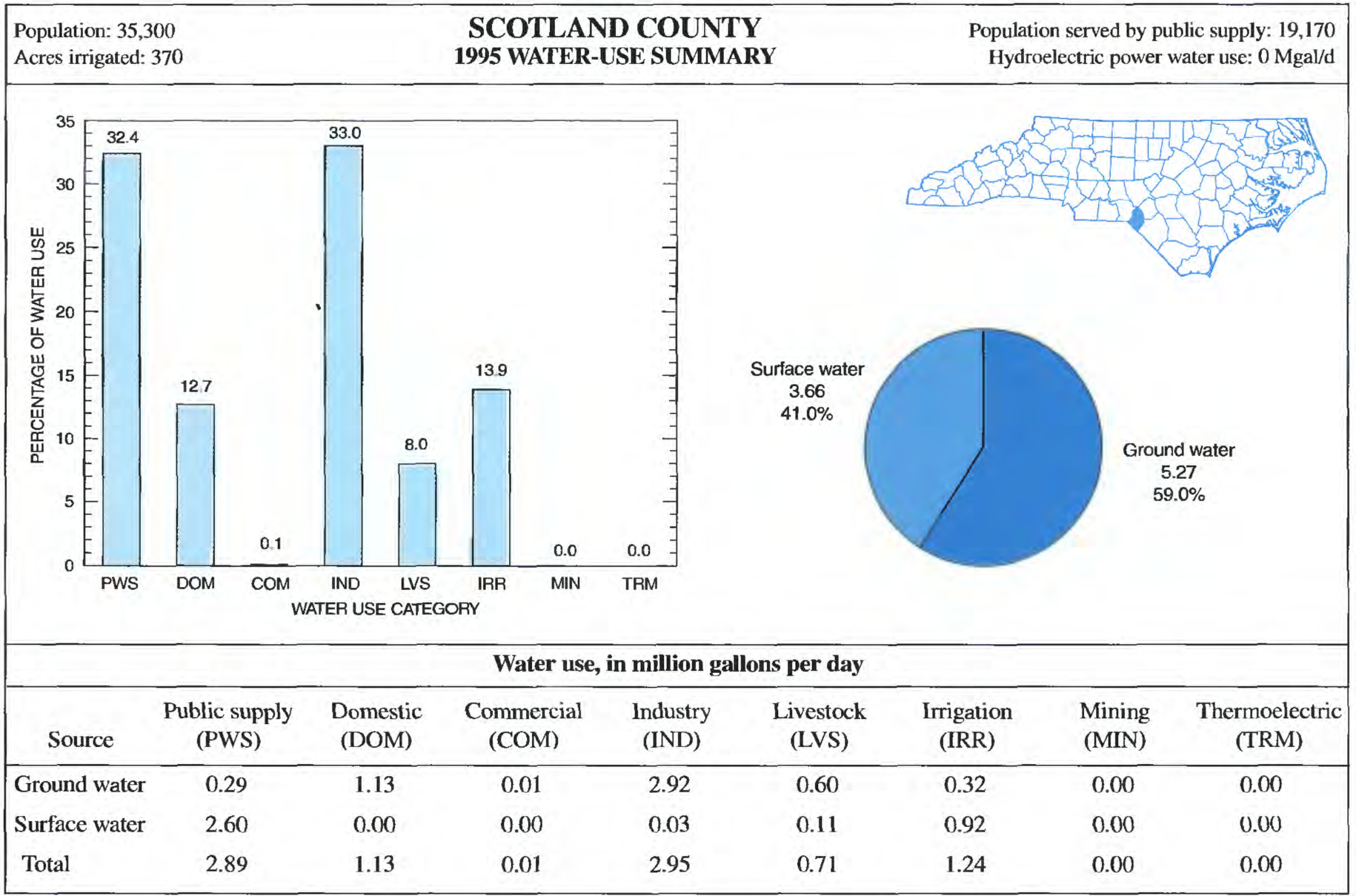

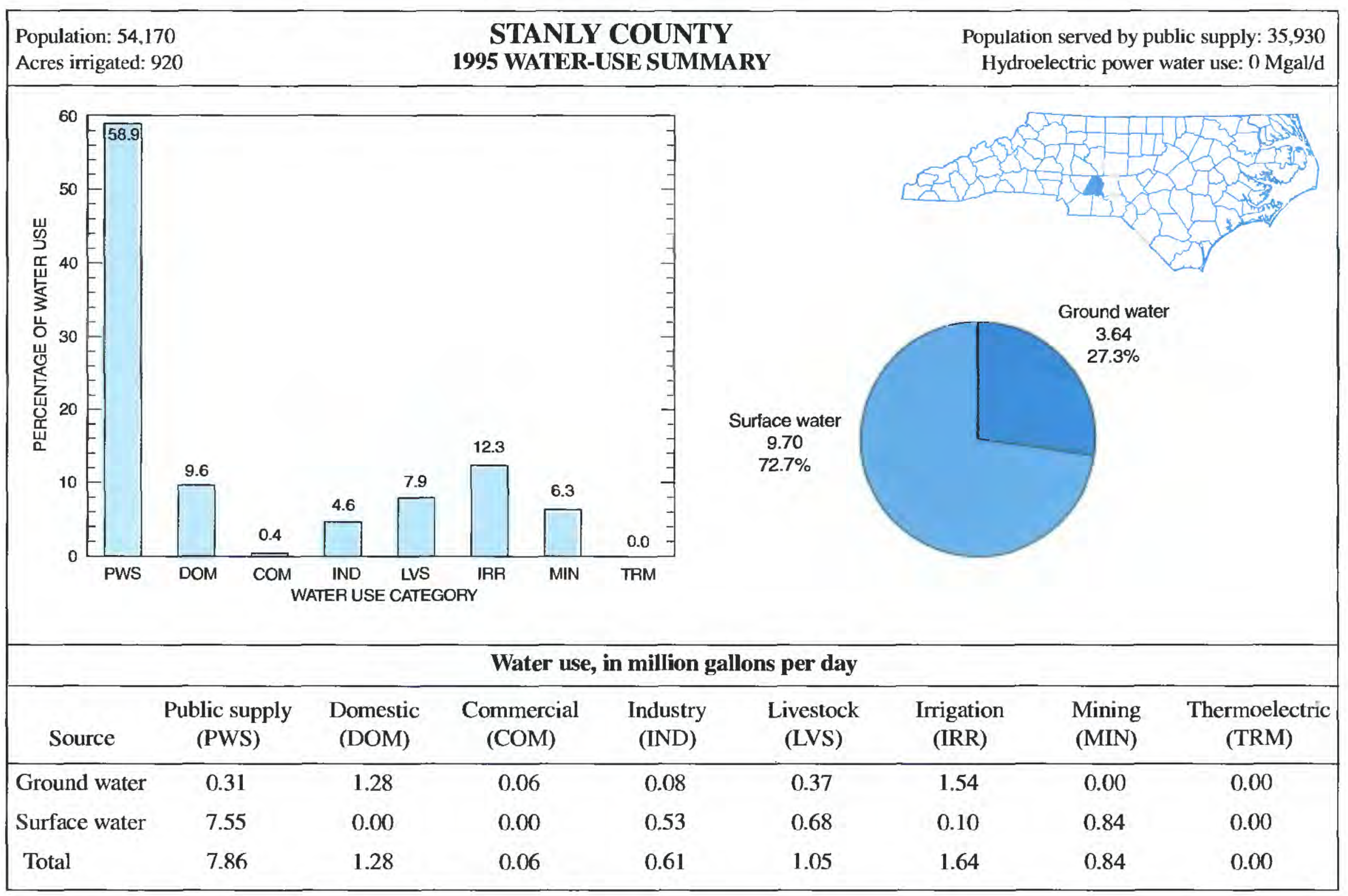




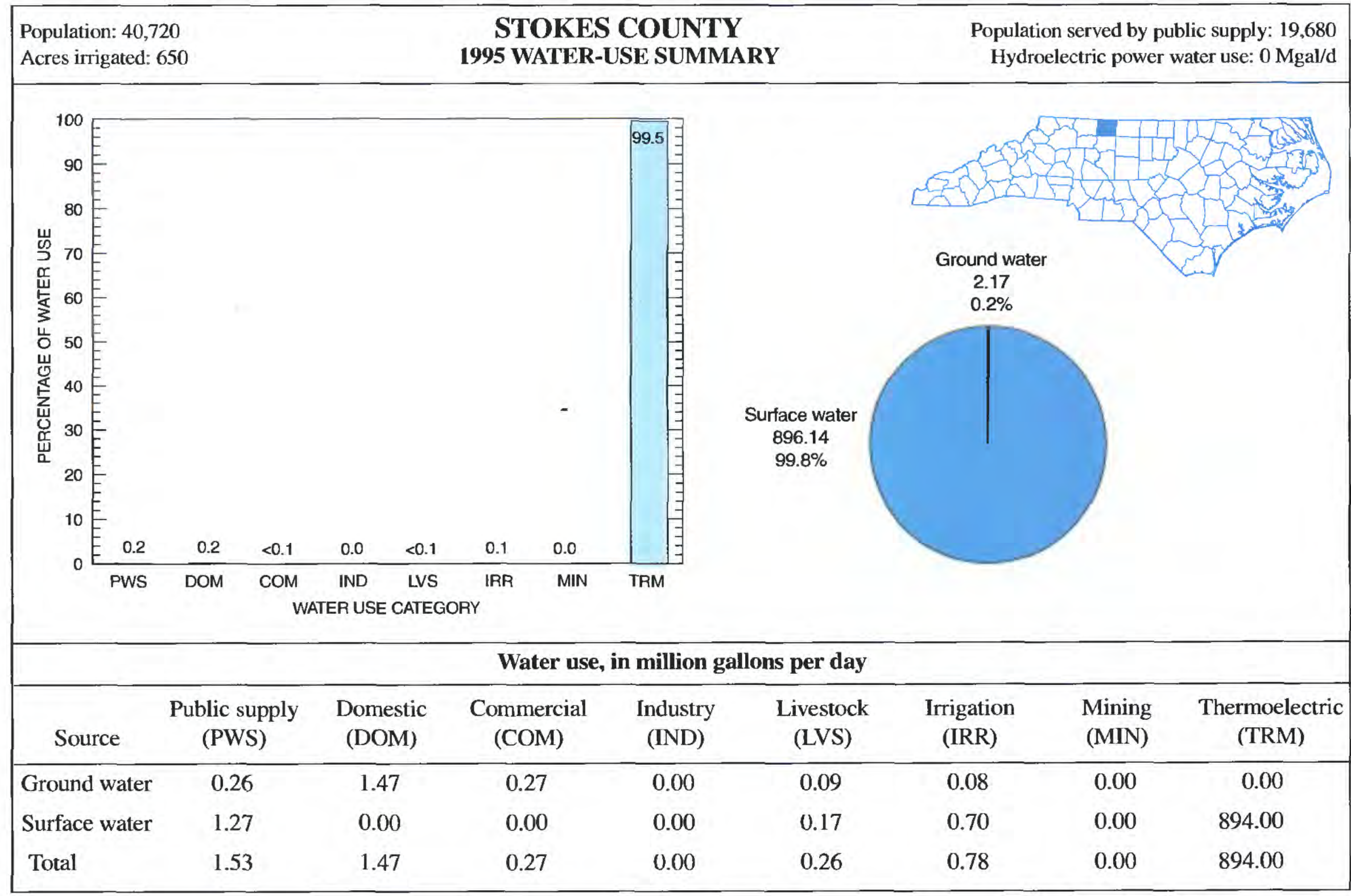

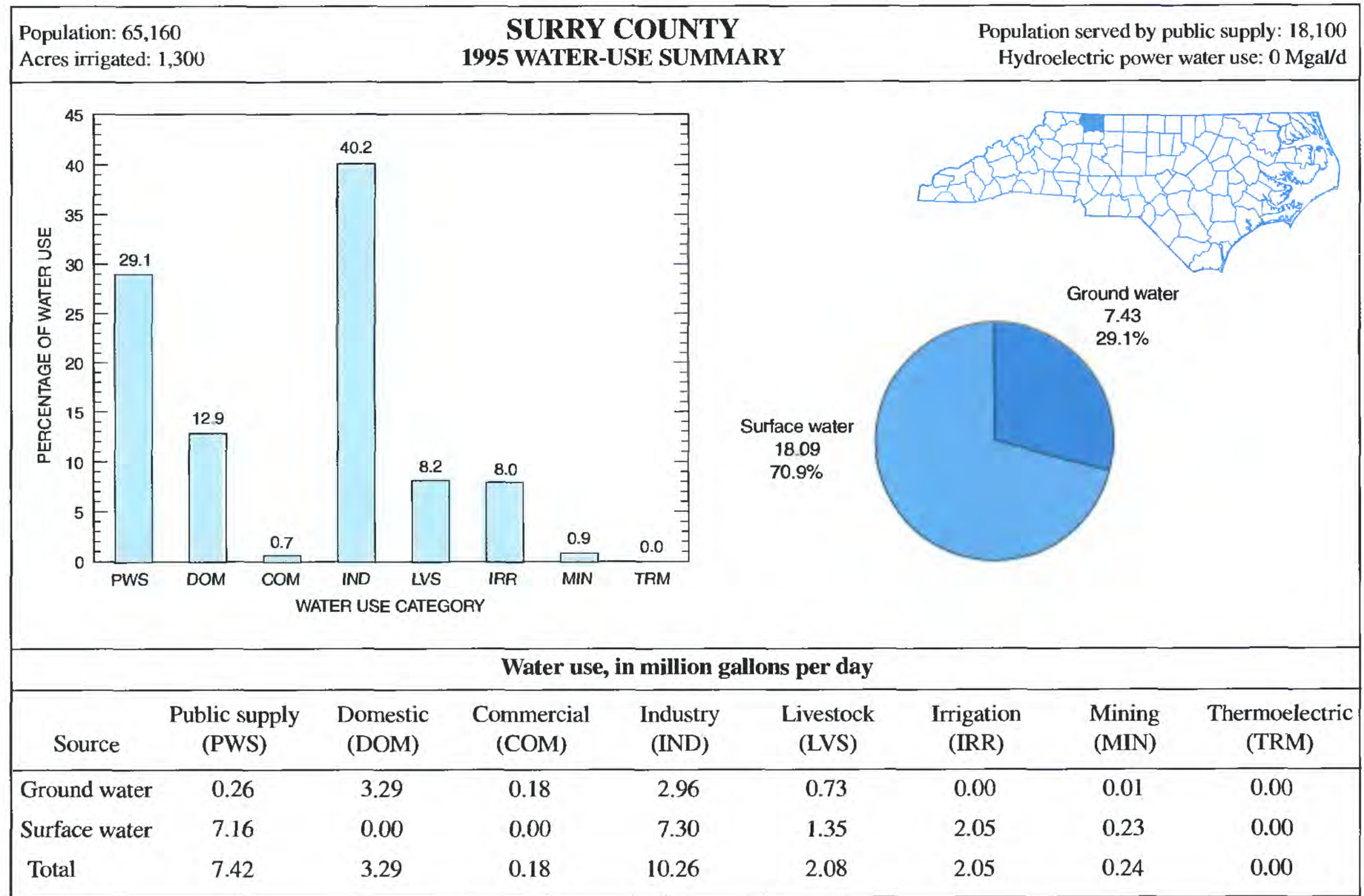




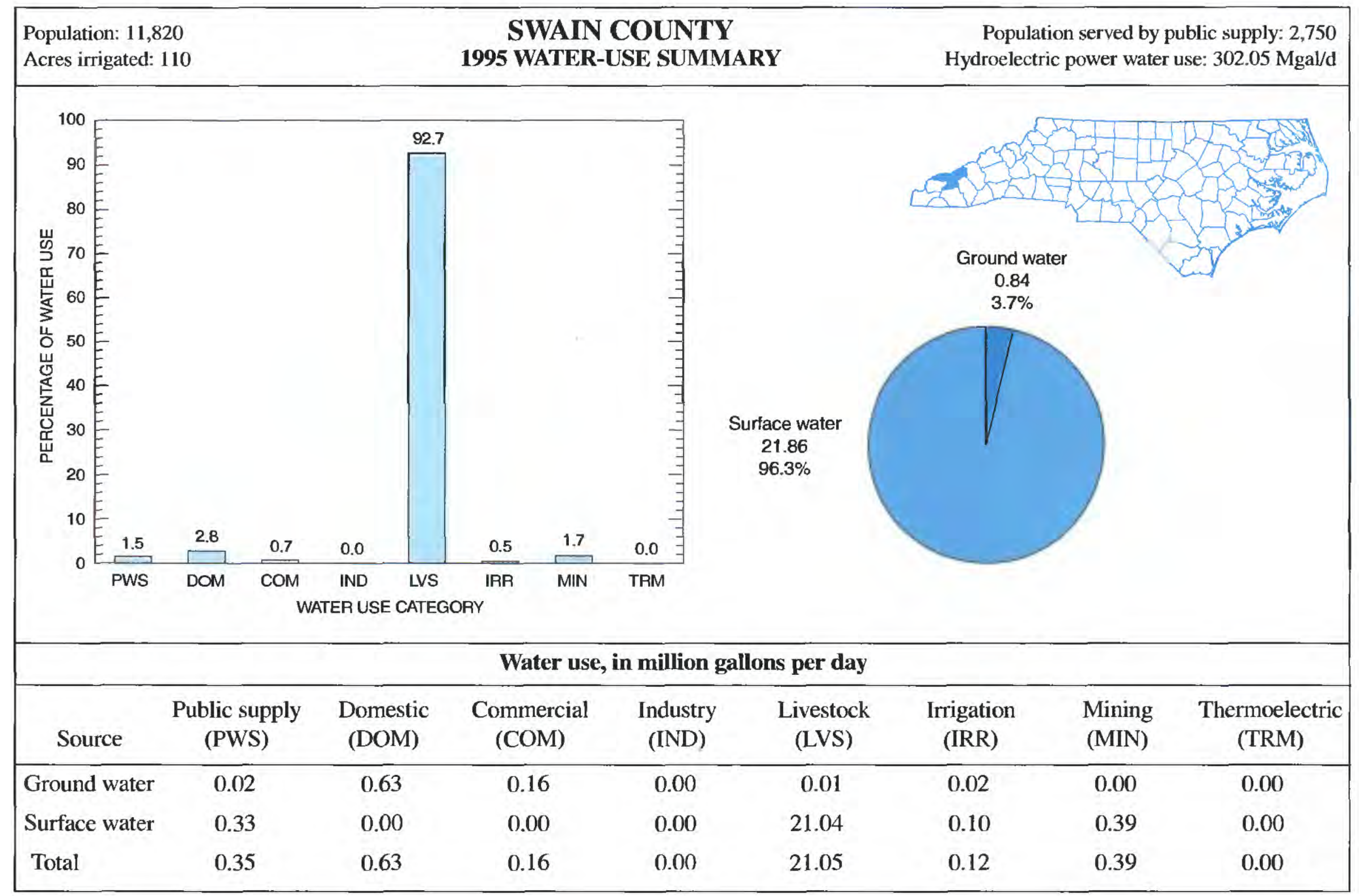

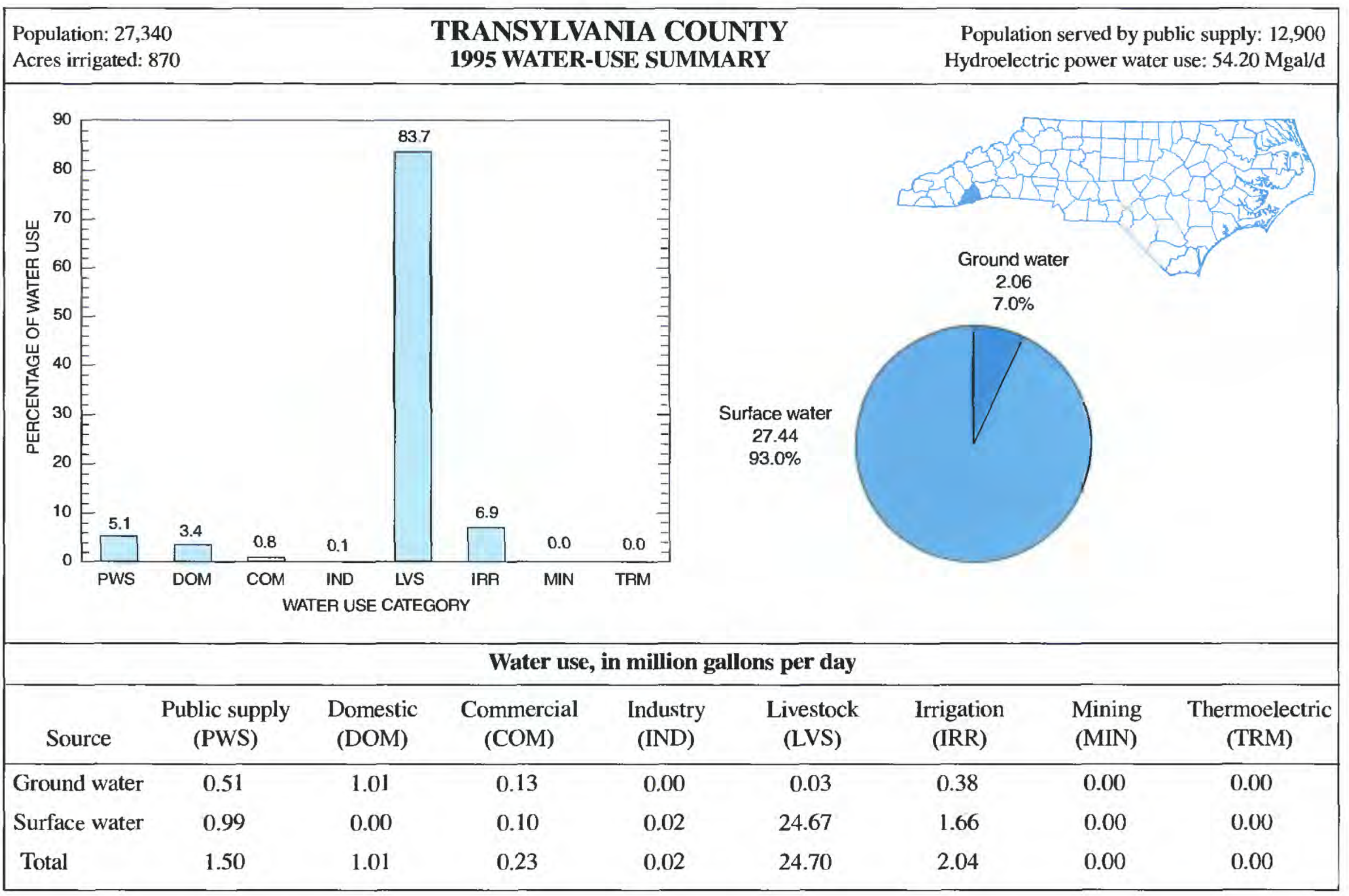




\begin{tabular}{|c|c|c|c|c|c|c|c|c|c|c|c|}
\hline \multicolumn{3}{|l|}{$\begin{array}{l}\text { Population: } 3,890 \\
\text { Acres irrigated: } 0\end{array}$} & \multicolumn{6}{|c|}{$\begin{array}{l}\text { TYRRELL COUNTY } \\
\text { 1995 WATER-USE SUMMARY }\end{array}$} & \multicolumn{3}{|c|}{$\begin{array}{l}\text { Population served by public supply: } 2,760 \\
\text { Hydroelectric power water use: } 0 \mathrm{Mgal} / \mathrm{d}\end{array}$} \\
\hline res & $\frac{1}{\text { DOM }}$ & $\begin{array}{c}0.0 \\
\mathrm{COM} \\
\mathrm{n}\end{array}$ & $\begin{array}{r}0.0 \\
\text { IND } \\
\text { ER US }\end{array}$ & $\begin{array}{l}\text { LVS } \\
\text { LATEC }\end{array}$ & $\begin{array}{l}0.0 \\
\text { IRP } \\
\text { IRY }\end{array}$ & $\begin{array}{l}0.0 \\
\text { MiN }\end{array}$ & 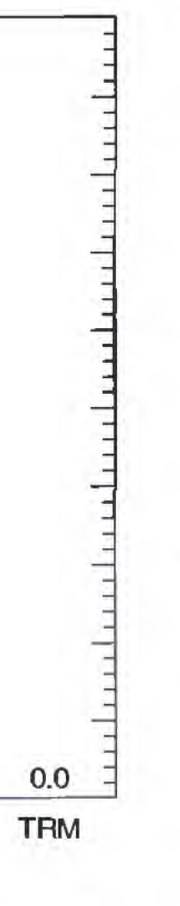 & $\begin{array}{c}\text { Surface water } \\
0.00 \\
0 \%\end{array}$ & & Gro & $\begin{array}{l}\text { d water } \\
51 \\
0 \%\end{array}$ \\
\hline \multicolumn{12}{|c|}{ Water use, in million gallons per day } \\
\hline Source & $\begin{array}{r}\text { Public } \\
\text { (P }\end{array}$ & $\begin{array}{l}\text { upply } \\
\text { S) }\end{array}$ & $\begin{array}{r}\text { Dor } \\
\text { (D }\end{array}$ & & $\begin{array}{l}\mathrm{Comm} \\
(\mathrm{CO}\end{array}$ & $\begin{array}{l}\text { rcial } \\
\text { 4) }\end{array}$ & $\begin{array}{c}\text { Industry } \\
\text { (IND) }\end{array}$ & $\begin{array}{l}\text { Livestock } \\
\text { (LVS) }\end{array}$ & $\begin{array}{l}\text { Irrigation } \\
\text { (IRR) }\end{array}$ & $\begin{array}{l}\text { Mining } \\
\text { (MIN) }\end{array}$ & $\begin{array}{l}\text { Thermoelectric } \\
\text { (TRM) }\end{array}$ \\
\hline Ground water & & & & & 0.0 & & 0.00 & 0.18 & 0.00 & 0.00 & 0.00 \\
\hline Surface water & & & & & 0.0 & & 0.00 & 0.00 & 0.00 & 0.00 & 0.00 \\
\hline Total & & & & & 0.0 & & 0.00 & 0.18 & 0.00 & 0.00 & 0.00 \\
\hline
\end{tabular}

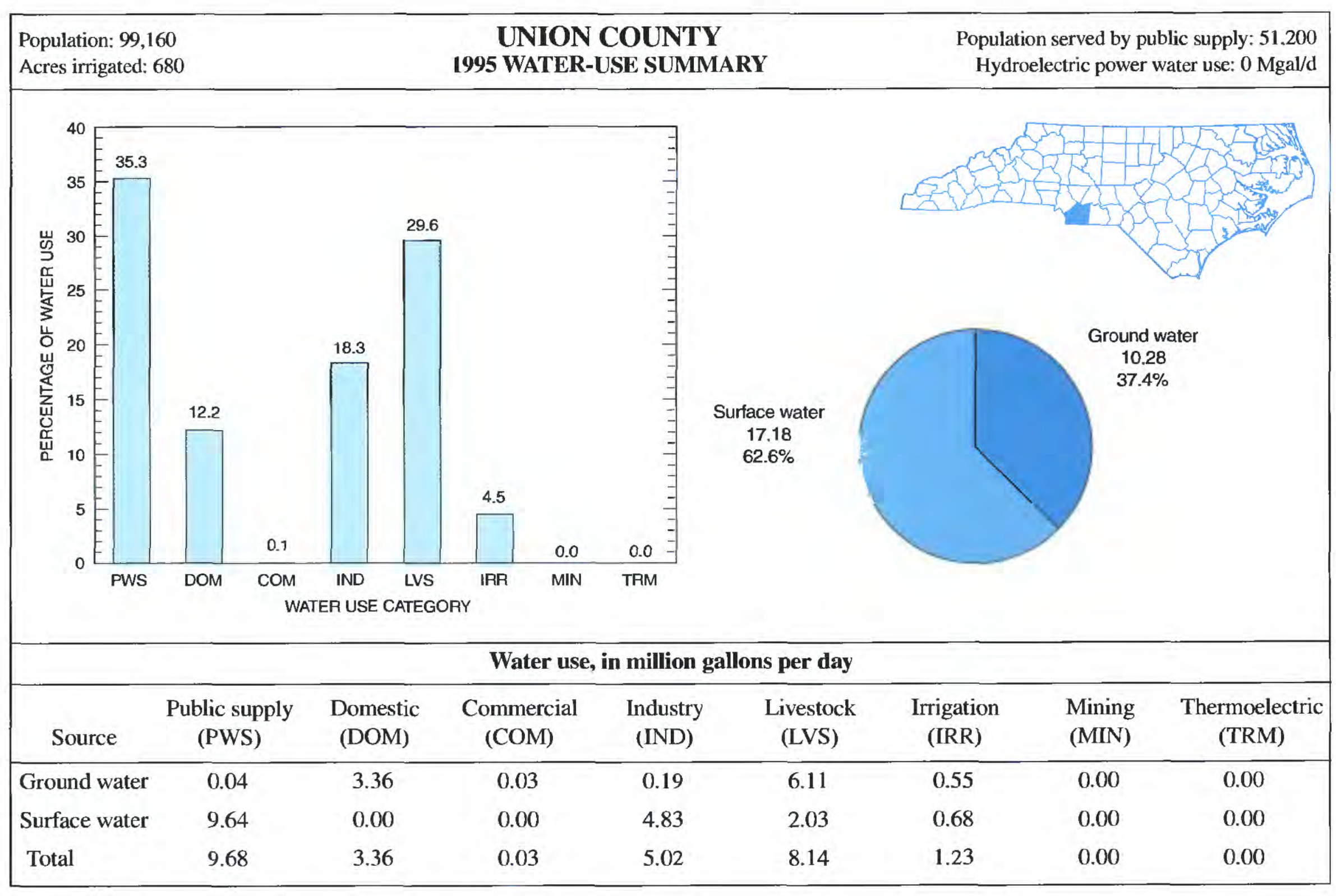




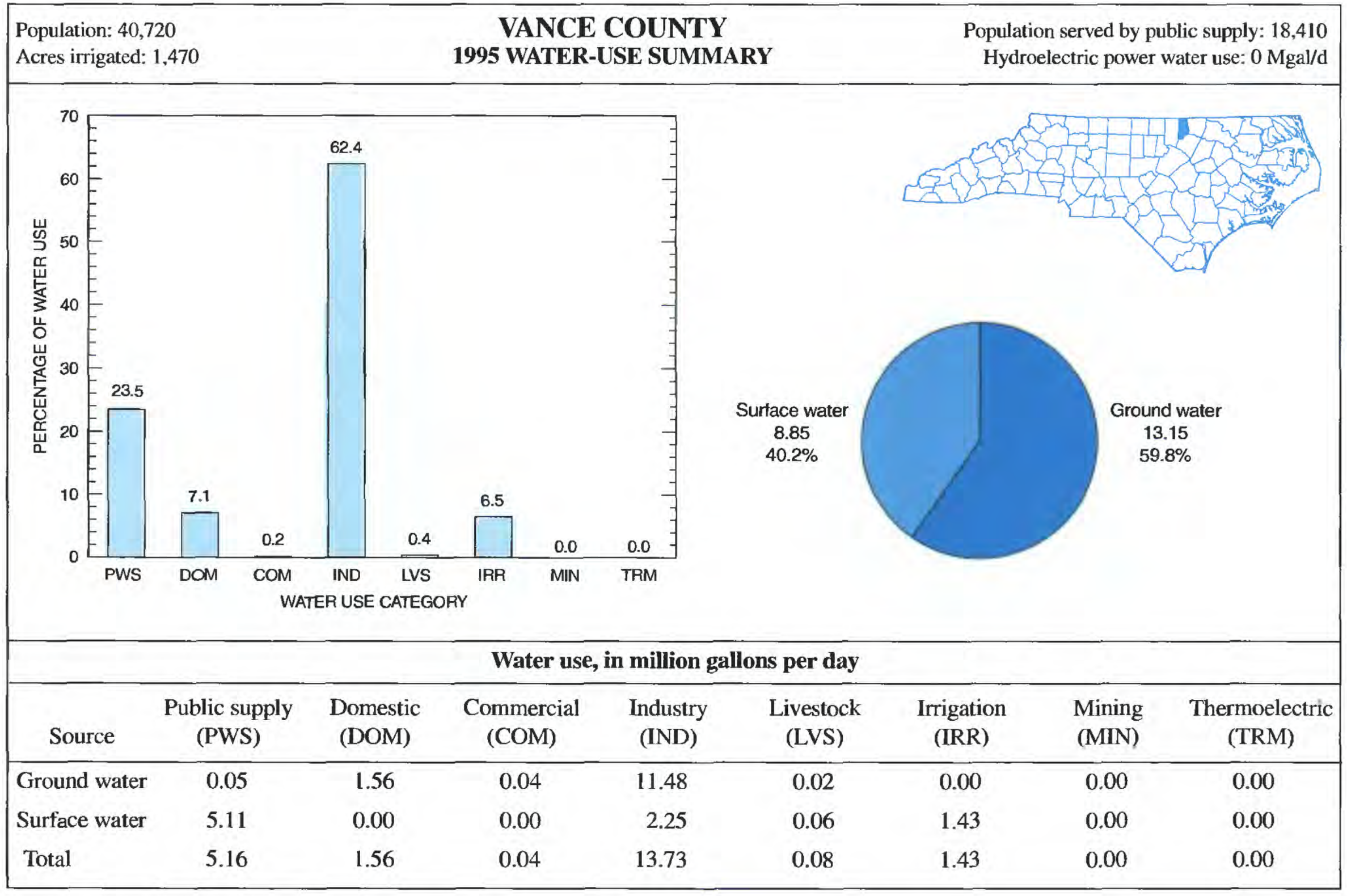

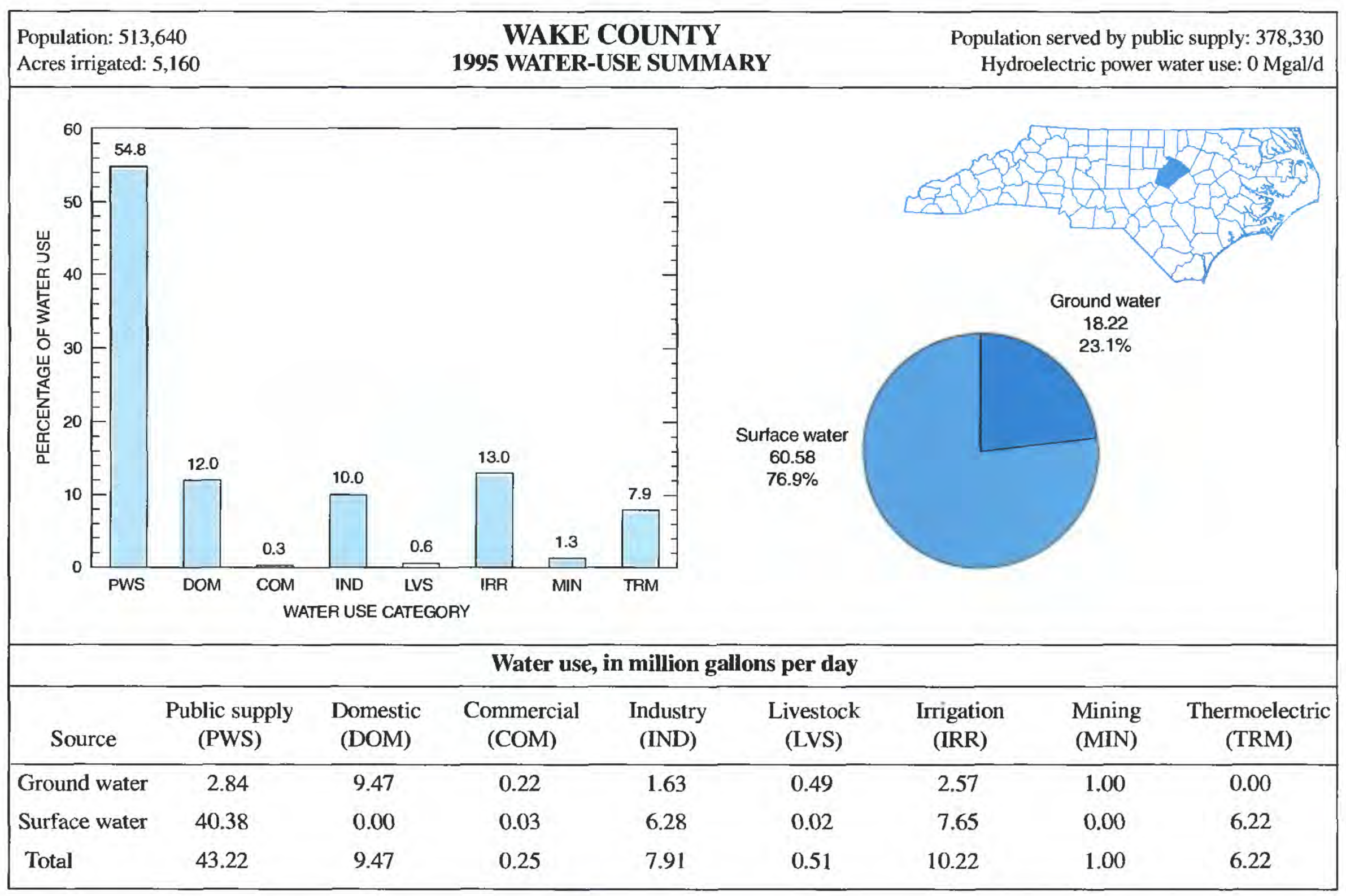




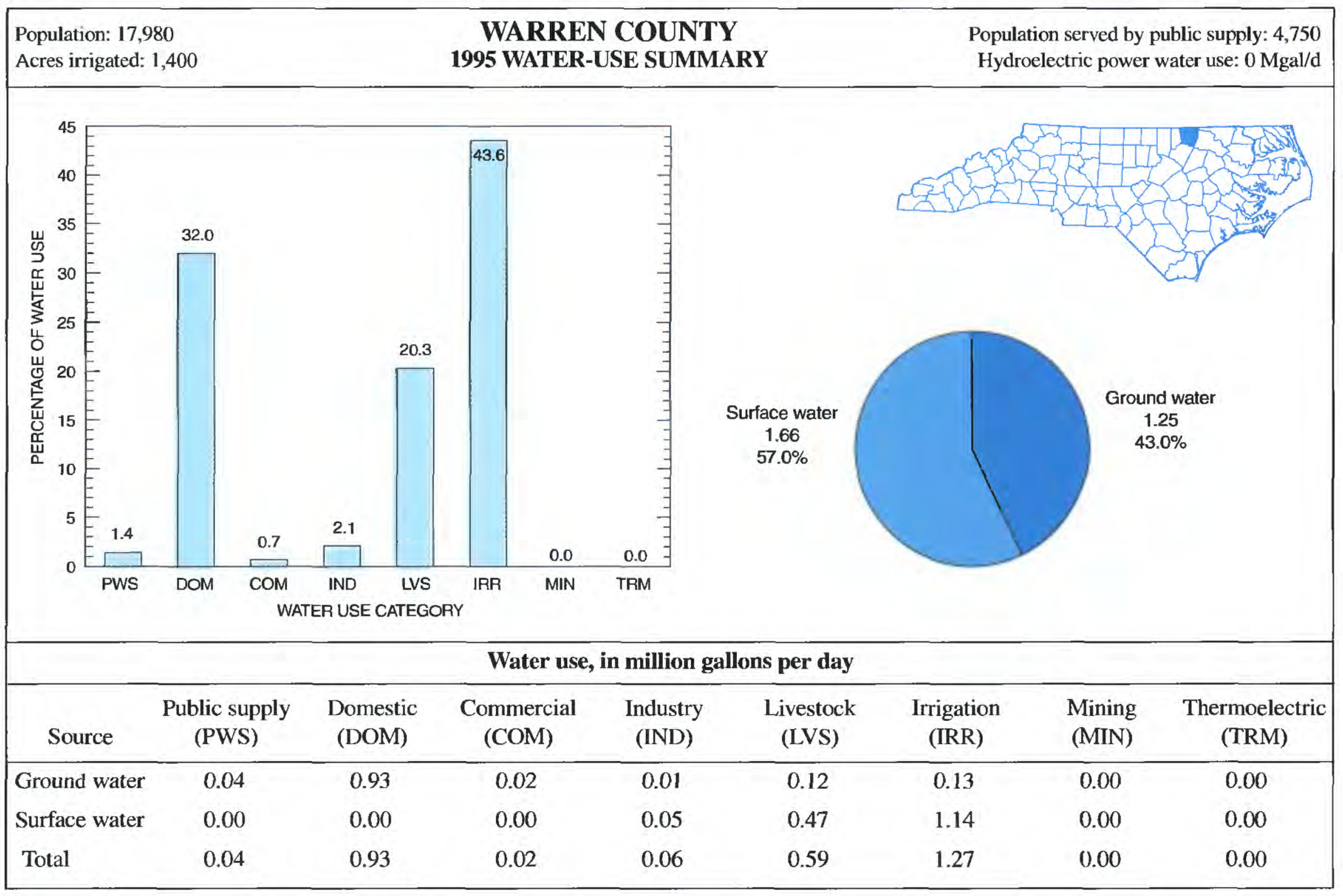

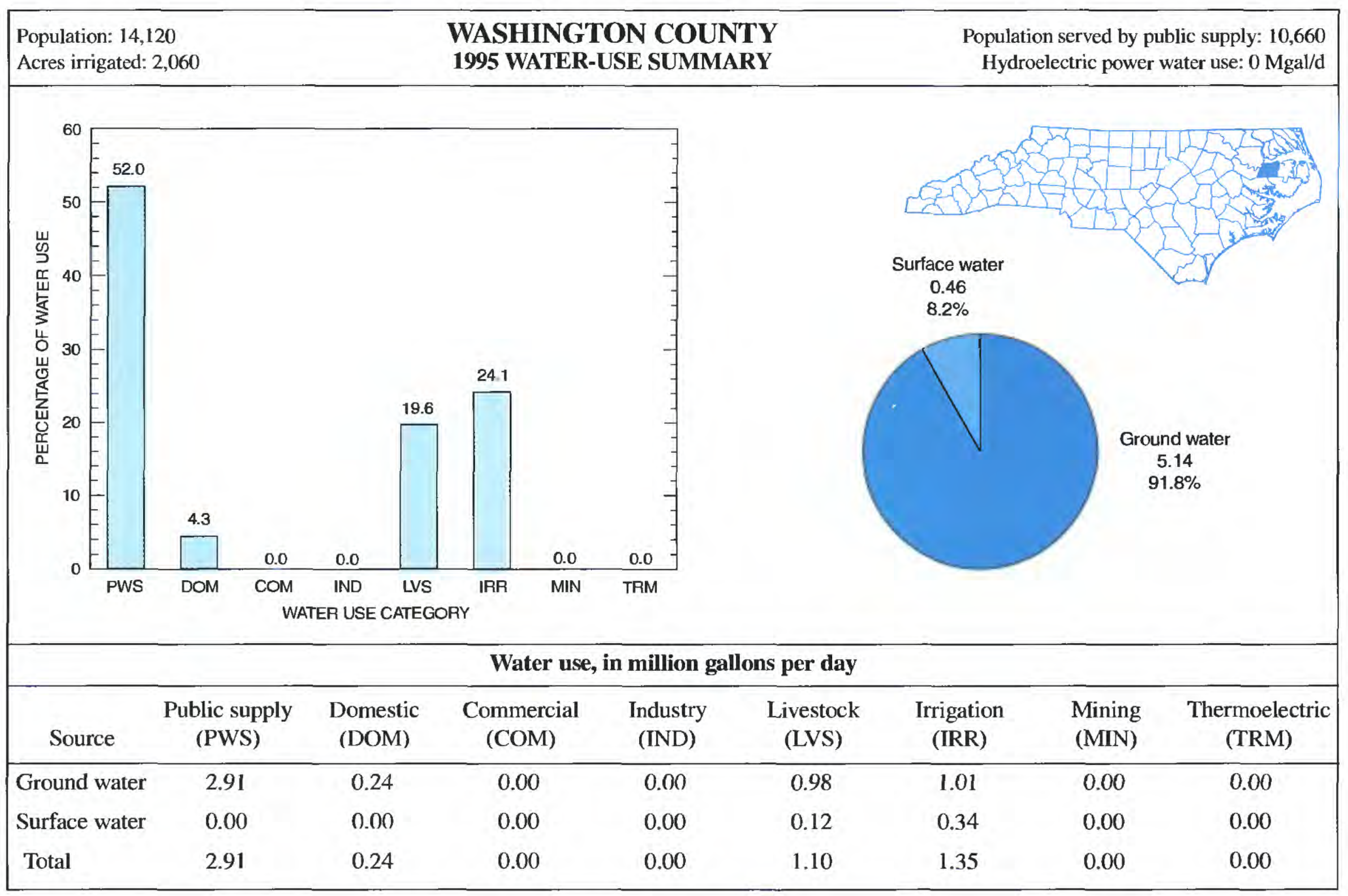




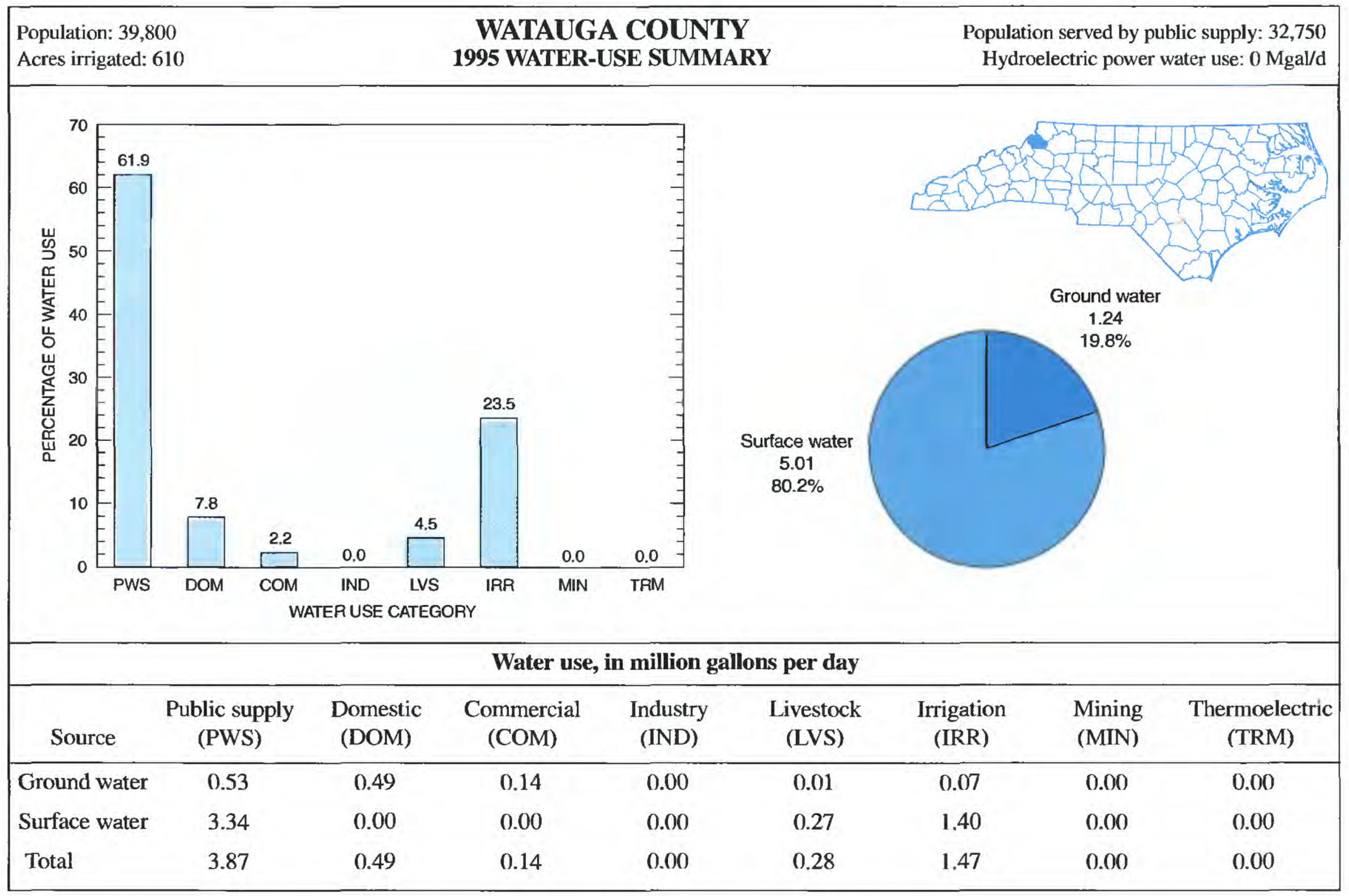

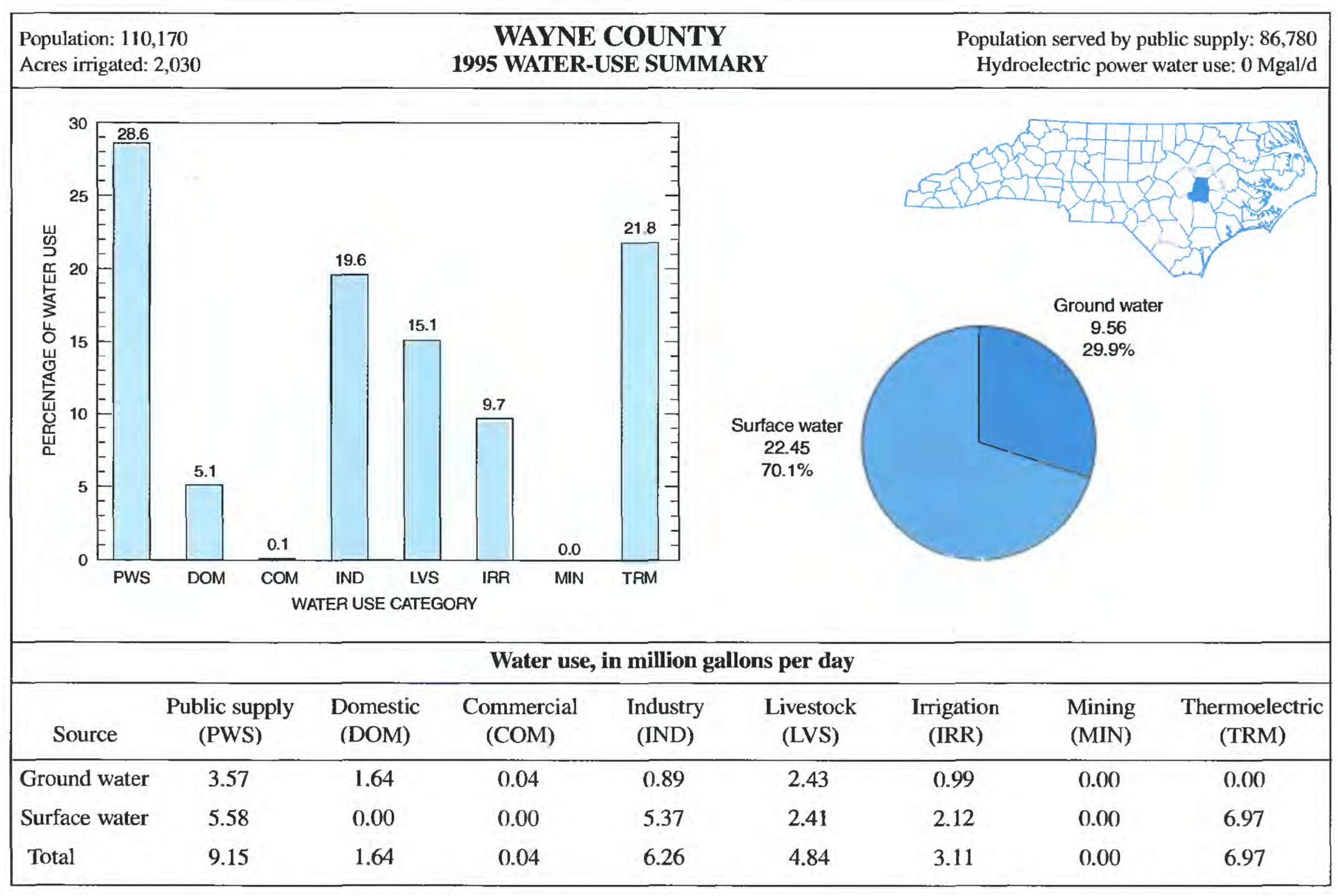




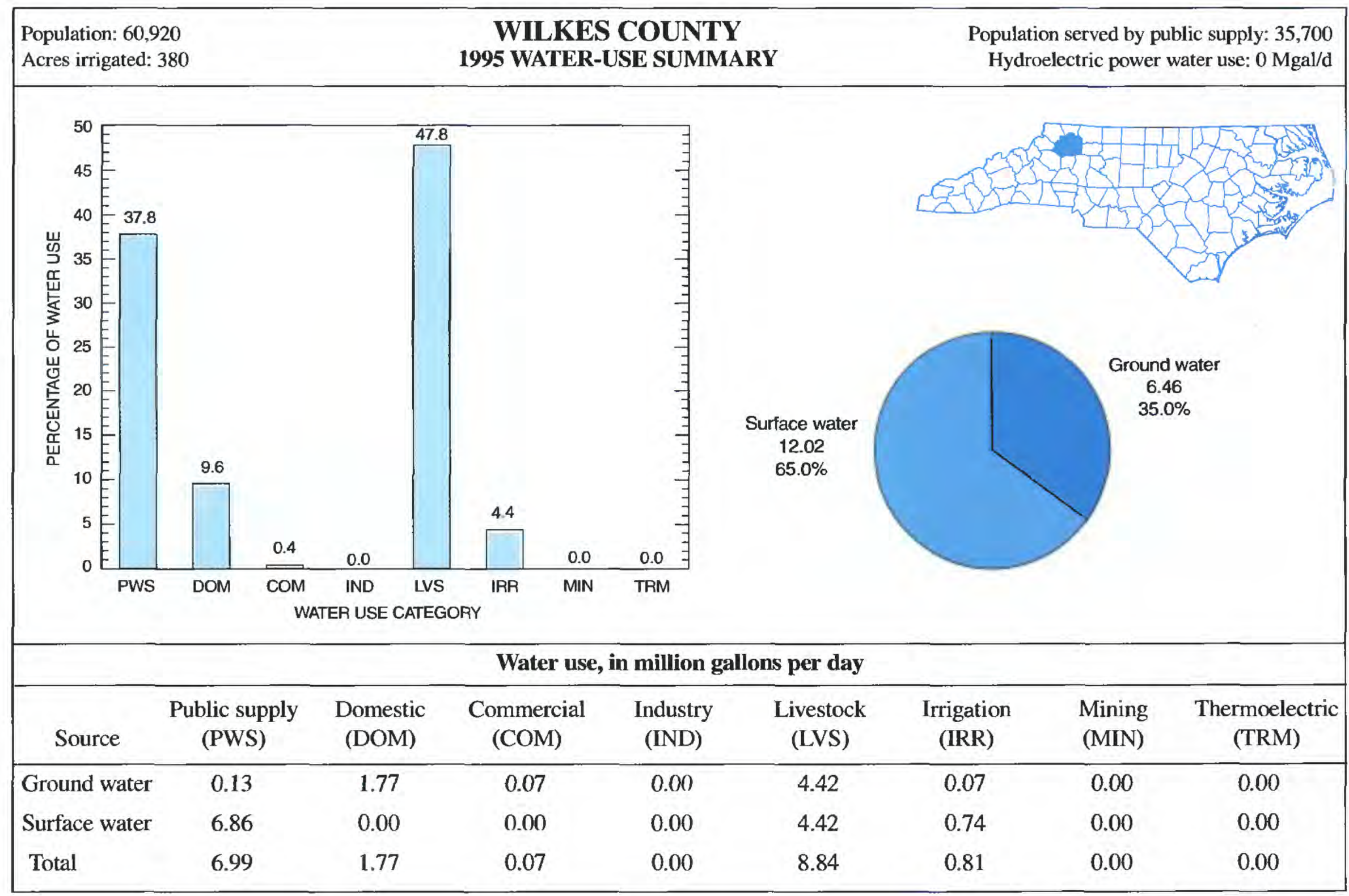

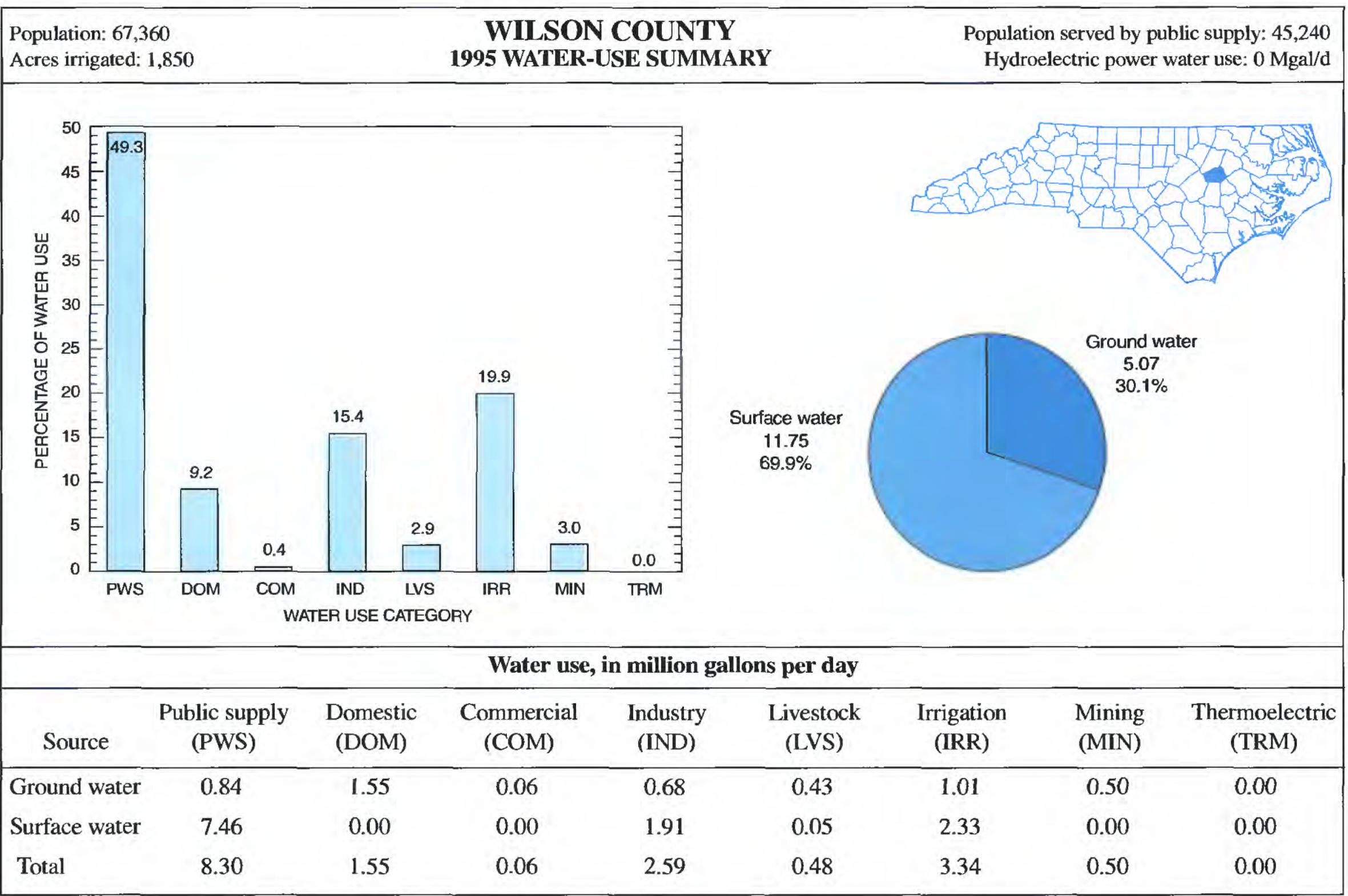




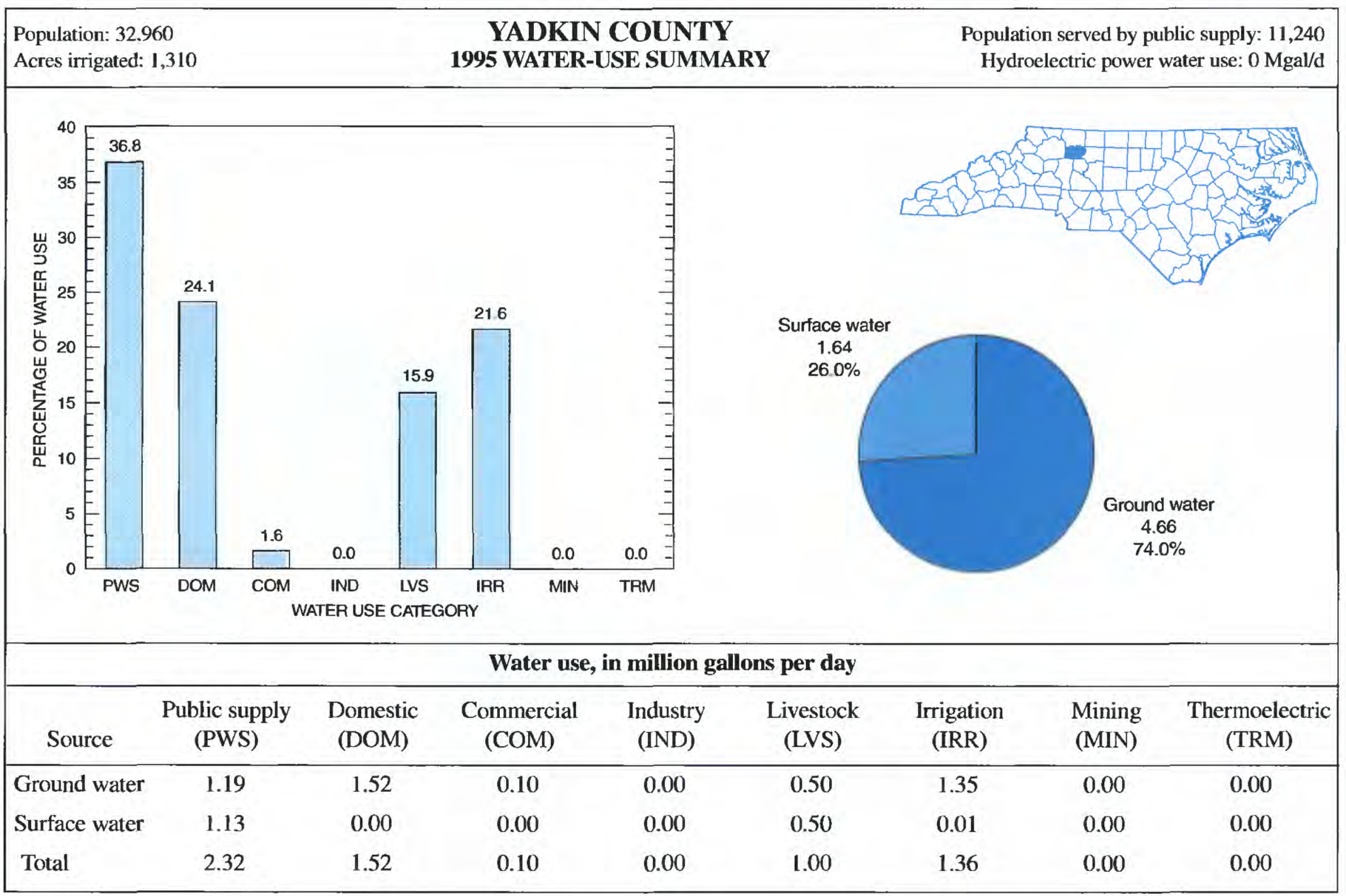

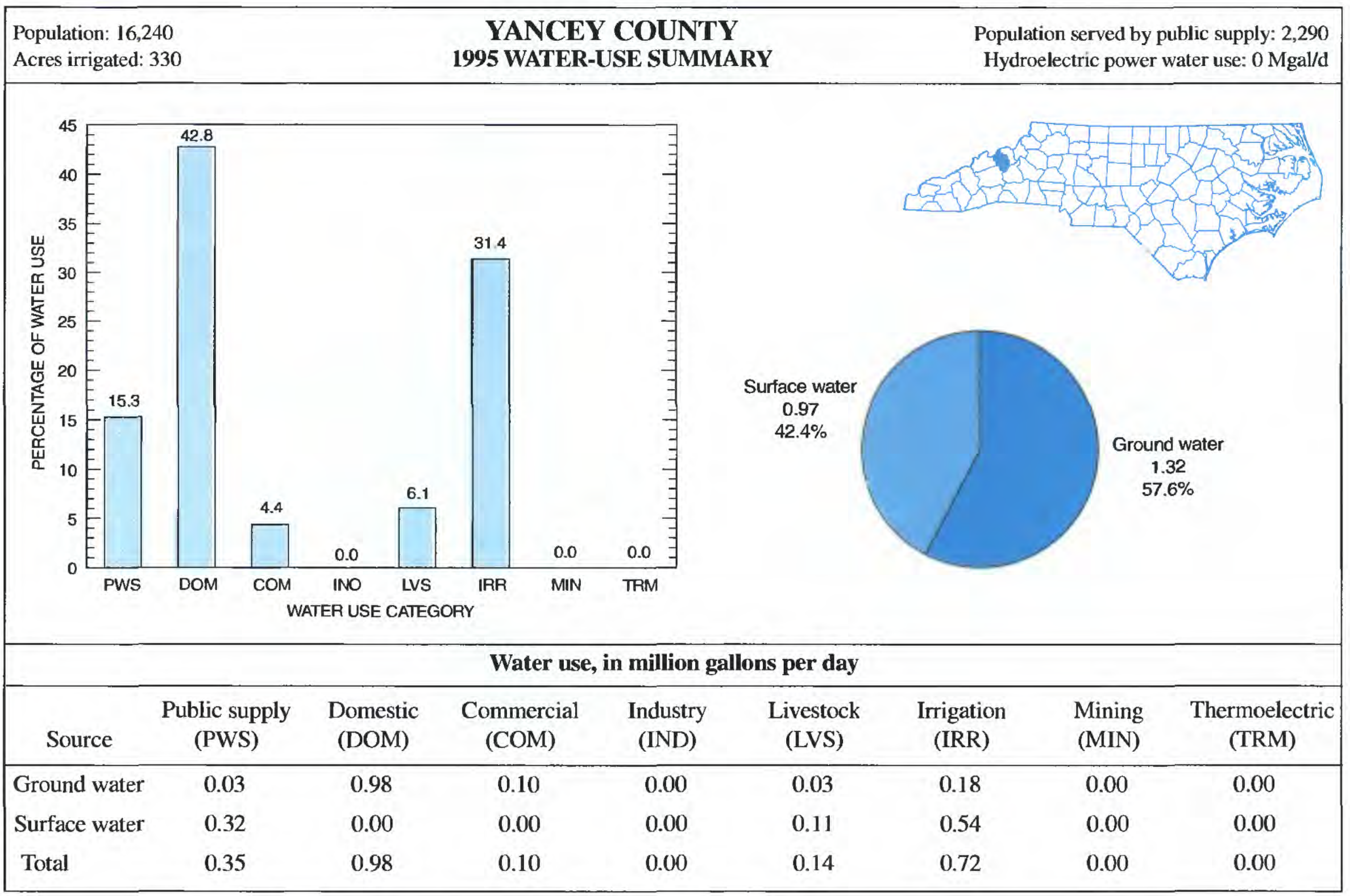




\section{GLOSSARY}

Acres irrigated-The total number of acres of crops that were irrigated during the year.

Animal specialties-Water use associated with the production of fish in captivity except fish hatcheries, fur-bearing animals in captivity, horses, rabbits, and pets.

Commercial water use-Water for motels, hotels, restaurants, office buildings, and other commercial facilities and institutions, both civilian and military. The water may be obtained from a public-supply facility or may be self-supplied. See also public supply and self-supplied water.

Community water system-A water supplier that furnishes water for at least 25 people, or that has a minimum of 15 hookups.

Consumptive use-That part of water withdrawn that is evaporated, transpired, incorporated into products or crops, consumed by humans or livestock, or otherwise removed from the immediate water environment. Also referred to as water consumed.

Cooling water-Water used for cooling purposes, such as for condensers and nuclear reactors.

Delivery-The amount of water delivered to the point of use.

Domestic population served-The total number of people served by the public suppliers during the calendar year.

Domestic water use-Water used for household purposes, such as drinking, food preparation, bathing, washing clothes and dishes, flushing toilets, and watering lawns and gardens. Also called residential water use. The water may be obtained from a public-supply facility or may be self-supplied. See also public supply and selfsupplied water.
Fossil-fuel power-Electrical power generated by using fossil-fuel (coal, oil, or natural gas) energy.

Freshwater-Water that contains less than 1,000 milligrams per liter $(\mathrm{mg} / \mathrm{L})$ of dissolved solids; generally, more than $500 \mathrm{mg} / \mathrm{L}$ of dissolved solids is undesirable for drinking and many industrial uses.

Gigawatt-hour (GWh)-One billion watt-hours.

Ground water-Generally all subsurface water as distinct from surface water; specifically, that part of the subsurface water in the saturated zone (a zone in which all voids are filled with water).

Hydroelectric-power generation water use- The use of water in the generation of electricity at plants where the turbine generators are driven by falling water; an instream use.

Industrial water use-Water used for industrial purposes, such as fabrication, processing, washing, and cooling, and includes such industries as steel. chemical and allied products, paper and allied products, and mining and petroleum refining. The water may be obtained from a public-supply facility or may be self-supplied. See also public supply and self-supplied water.

Instream use--Water use taking place within the stream channel for such purposes as hydroelectric-power generation, navigation, water-quality improvement, fish propagation, and recreation. Sometimes called non-withdrawal use or in-channel use.

Irrigation water use-Artificial application of water to lands to assist in the growing of crops and pastures or to maintain vegetative growth in commercial nurseries and recreational lands, such as parks and golf courses.

Kilowatt-hour (KWh)-One thousand watt-hours.

(Continued On Inside Back Cover)

COVER PHOTOGRAPH: Tristan Walters helping his brother Braden to a cool drink of water on a hot day at the pool. 
Livestock water use-Water for stock watering, feed lots, dairy operations, fish farming, and other on-farm needs. Livestock as used here includes cattle, sheep, goats, hogs, and poultry. Also included are such animal specialties as horses, rabbits, bees, pets, fur-bearing animals in captivity, and fish in captivity.

Million gallons per day (Mgal/d)--A rate of flow of water.

Mining water use - Water use for the extraction of minerals occurring naturally, including solids, such as coal, clay, and ores; liquids, such as crude petroleum; and gases, such as natural gas. Also includes uses associated with quarrying for rock aggregates, such as sand and gravel, and well operations, milling (crushing, screening, washing, floatation, and other), and other preparations customarily done at the mine site or as part of a mining activity.

Noncommunity water system-A water supplier that furnishes water for less than 25 people and has less than 15 hookups.

Nuclear power-Electrical power generated using nuclear energy.

Offstream use-Water withdrawn and diverted from a ground- or surface-water source for public supply, domestic, commercial, industry, irrigation, livestock. thermoelectric-power generation. and other uses. Sometimes called off-channel use or withdrawal use.

Per capita use-The average amount of water used per person within a given unit area during a standard time period, generally per day.

Per capita withdrawals - The average amount of water withdrawn per person within a given unit area during a standard time period, generally per day.

Public supply - Water withdrawn by public and private water suppliers and delivered to groups of users. Public suppliers provide water for a variety of uses, such as domestic, commercial, thermoelectric power, industrial, and public water use. See also commercial water use, domestic water use, industrial water use, and public water use.
Public use and losses-The use of water for municipal functions, such as firefighting, street cleaning, and recreation, and losses resulting from the transfer of water and conveyance losses associated with leaky pipes in the distribution systems.

Public water use-Water supplied from a public supply and used for such purposes as firefighting, street washing, and municipal parks and swimming pools. See also public supply.

Reclaimed wastewater-Wastewater-treatment plant effluent that has been diverted or intercepted for reuse before it reaches a natural waterway or aquifer.

Saline water-Water that contains more than $1,000 \mathrm{mg} / \mathrm{L}$ of dissolved solids.

Self-supplied water-Water withdrawn from a ground- or surface-water source by a user rather than being obtained from a public-supply facility.

Spray irrigation-Artificial application of water by spray techniques to lands to assist in the growing of crops and pastures or to maintain vegetative growth in recreational lands.

Stock-Water use associated with the production of cattle, sheep, goats, hogs, and poultry. See also livestock water use.

Surface water-An open body of water, such as a stream or a lake.

Thermoelectric-power generation water use-Water used in the process of the generation of thermoelectric power. The water may be obtained from a publicsupply facility or may be self-supplied. See also public supply and self-supplied water.

Watt-hour (Wh)-An electrical energy unit of measure equal to one watt of power supplied to or taken from an electrical circuit continuously for one hour.

Withdrawal-The amount of water withdrawn from a water source (ground or surface, fresh or saline). This is equivalent to "intake," "water diversion," or "pumpage," terms commonly used by industry, and for irrigation, and public supply, respectively. See also offstream use and self-supplied water. 